


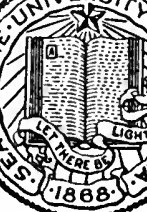

THE LIBRARY OF

\section{THE UNIVERSITY OF CALIFORNIA LOS ANGELES}




\section{OPERATIVE DENTAL SURGERY}


Digitized by the Internet Archive in 2007 with funding from. Microsoft Corporation 


\section{OPERATIVE}

\section{DENTAL SURGERY}

BY

\section{THOMAS GOODHUGH}

LECTURER ON OPERATIVE DENTAE SURGERY TO THE INCORPORATED DENTAL SOCIETY

\section{WITH 96 ILLUSTIRATIONS}

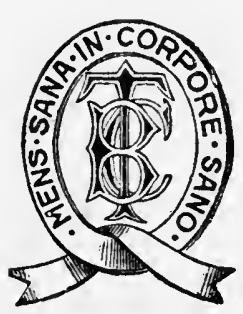

LONDON

BAILLIERE, TINDALL AND COX 8, HENRIETTA STREET, COVENT GARDEN 


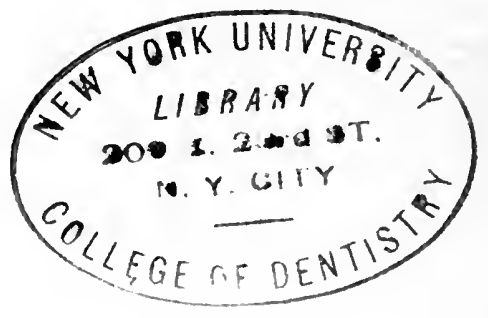

Printed in Great Britain 


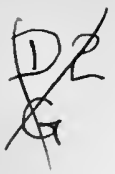

75367

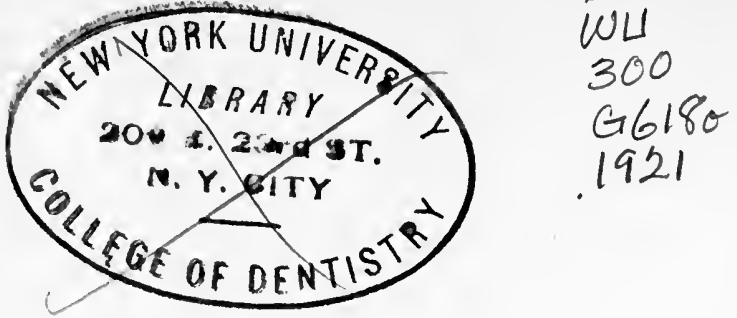

To

THE ART OF DENTISTRY

TO WHICH THE WHOLE OF MY LIFE HAS

BEEN DEVOTED 



\section{PREFACE}

THis work is based upon a long series of dental lectures which I have delivered to the members of the Incorporated Dental Society, in London and various cities and towns throughout $G$ reat Britain, during a period of fourteen years. It has been written at the suggestion of Mr. F. Butterfield, Honorary Secretary of the Society, whose exceptional organizing abilities have resulted in the public acknowledgment of that institution as being beneficial to the practice of dentistry and to the public of this country.

The lectures were designed to bring to chairside work such medical and surgical principles as would ensure that practical operative procedure be firmly based upon true knowledge.

I am deeply indebted to the many dental and medical works which have been so comprehensively written by authorities on these subjects, as the main source of my knowledge, coupled with extensive practical investigation of any sections bearing on the art of dentistry, and to constant visits to the Royal College of Surgeons' Museum and the South Kensington Natural History Museum; also to the many courtesies extended by many medical and dental practitioners during a period of over twenty years:

The large number of specimens at my disposal have been collected during a number of years, and have provided illustrations for the work. Thirteen of them I drew myself and the remainder I specially prepared for the photographer.

To the authors of the books which I have selected as the basis of many of my lectures I desire to express full indebtedness. Lists of these works are given in the course of the chapters on various subjects. Especially would I mention Marshall's "Operative Dentistry," Halliburton's "Physiology," Gray's "Anatomy," Cunningham's " Anatomy," Holden's "Osteology," Blair's "Surgery of the Mouth and Jaws," and Prinz's "Dental Materia Medica." 
The practical sections are the result of my personal experience covering a period of thirty years.

I wish also to acknowledge the courtesy of the Mouth Mirror for the loan of two blocks of a child's skull which were originally prepared by $\mathrm{Mr}$. G. Houlson for illustrating an article in that journal ; to Mr. H. Clifford Smith for the loan of a disarticulated skull and a fœetal skull for photographic purposes ; to Mr. J. Sefton Sewill for his very kind permission to use twenty-three microphotographic illustrations taken from his father's work on "Dental Surgery"; and also to Messrs. Baillière, Tindall and Cox for the help they have given me in preparing this work.

THOMAS GOODHUGH.

LONDON,

August, r921. 


\section{CONTENTS}

CHAPTER

PAGE

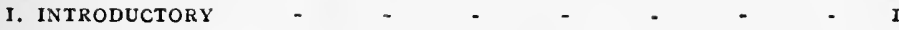

II. A DESCRIPTION OF EMBRYONIC DENTITION - $\quad$ - - $\quad$ - I3

II." THE STRUCTURAL FORMATION OF THE TEETH -

IV. $\Lambda$ DESCRIPTION OF THE IMMEDIATE NEIGHBOURING PARTS OF THE TEETH

V. A DESCRIPTION OF THE IMMEDIATE NEIGHBOURING PARTS OF THE TEETH (continued). THE CIRCULATORY SYSTEM - - - 66

VI. THE CIRCULATORY SyStem (continued). VEINS, BLOOD, BLOOD. PRESSURE, AND GASEOUS INTERCHANGE WITH RELATION TO ASPHYXIA

VII. THE CIRCULATORY SYSTEM (continned). THE IMMUNITY OF THE BLOOD AND ITS COAGULATIVE PROFERTIES, WITH RELATION TO ASEPSIS AND H.EMORRHAGE

- 96

VIII. THE NERVOUS SYSTEM FROM A DENTAL POINT OF VIEW - - IO6

1X. THE PARTS OF THE NERVOUS SYSTEM WHICH ARE DIRECTLY INVOLVED IN DENTAL OPERATING $\quad$ - $\quad$ - $\quad$ - $\quad$ - II9

X. THE MUSCLES AND ORGANS IN THE IMMEDIATE VICINITY OF THE

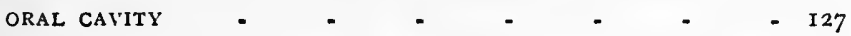

XI. THE CAUSES OF INFLAMMATORY AND SEPTIC DISEASES OF THE

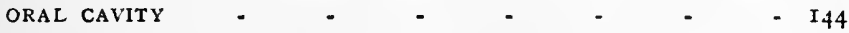

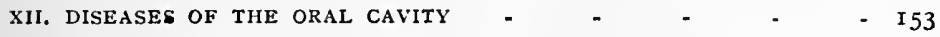

XIII. DENTAL MATERIA MEDICA AND THERAPEUTICS - - I - II

XIV. LOCAL AN.æSTHESIA OR ANALGESIA - $\quad$ - $\quad-\quad$ - $\quad$ - 190

XV. TIIE ADMINISTRATION OF NITROUS OXIDE GAS ALONE, OR IN COMBINATION WITH AIR OR OXYGEN, FOR DENTAL OPERATIONS- 202

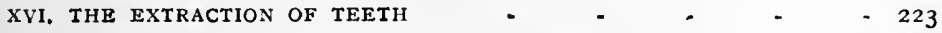

XVII. THE CARE AND TREATMENT OF CHILDREN'S TEETH - 234

XVIII. THE PREPARATION OF CAVITIES IN TEETH, AND FILliNg With

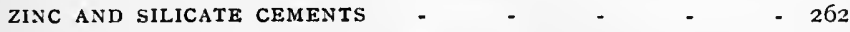

XIX. THE FilliNg OF CAVIties With aMALGaM AND GOLD - $\quad 279$

XX. ROOT TREATMENT AND FILLING - $\quad$ - $\quad$ - $\quad$ - $\quad 298$

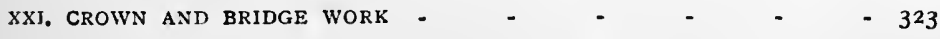

INDEX - 



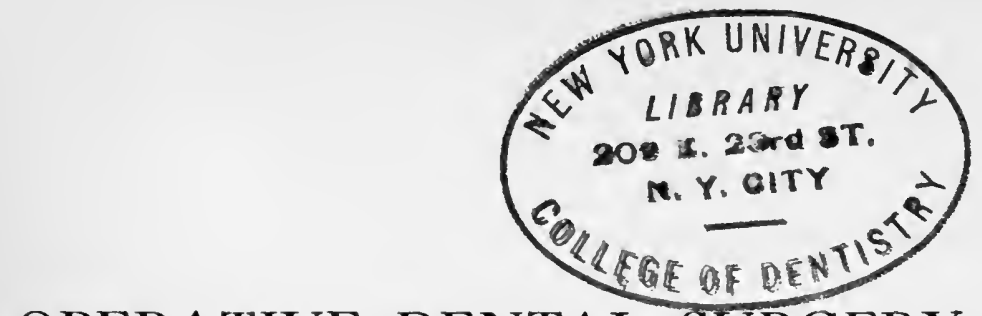

\section{OPERATIVE DENTAL SURGERY}

\section{CHAPTER I \\ INT RODUCT ORY}

SHOWING THE NECESSITY OF AWAKENING OUR CONCEPTION AND REALIZATION OF THE PHYSICAL AND CHEMICAL FORCES INVOLVED, AND THE UTILIZATION OF EVERYTHING THAT MAY BE MADE USEFUL IN THE APPLICATION OF OUR NIETHODS IN OUR WORK.

THE history of the origin, growth, and development of the processes of dentistry has been marked by more or less definite periods when certain methods and the materials involved have passed through the crucible of practical experience. The valuable principles, when grasped, have been retained, carried onward, and applied to the use of fresh materials. So true is this, that the practical methods of dentistry in use to-day can produce a high degree of perfection both in utility, natural appearance, and comfort.

The work of many French, German, and English dentists during the seventeenth and eighteenth centuries was performed with materials and instruments of meagre quality and variety. The nineteenth century was a period of great activity and progress in the production of materials, instruments, and methods.

Examples of these early practitioners undoubtedly laid the steps by which progress has been made, but unlike the art of painting, examples of which can be seen in the various art treasuries of the world beaming out with undying age, the light that guides succeeding students to imitate their principles of drawing, colouring, and shading. If only their secrets of mixing their colours to produce permanent effects could be shown too, what a heritage this ancient art could bestow to its devotees in coming ages! But yet we must not be hasty in regretting that the dental art has been slow in progress. We must remember that most of the arts are fascinating to the lay mind. From the 
earliest years many children have been father to the man in the display of their natural genius and intuitive talent which has laid the foundation of a future brilliant career, and inspired the tenacity of purpose, patience over details, and the enduring devotion which has built up a majestic monument of the history of their art. By years of learning, ending with complete mastery of known details, the true artist always strives to add with deep humility, yet proud affection, some addition to that noble structure of art, fully realizing that all arts are built up in the same way and that no man dare claim superiority over his fellowcraftsmen while he owes so much to those who have gone before him and provided the knowledge which after all he has only taken the trouble to find out, gather, devour, and make his own.

\section{Valuable Knowledge provides Enduring Material to build up Science and Art.}

The seeds of true knowledge grow; truth, like precious gems or purest gold, becomes impacted in a mine of the aged dust of erudition to be brought out, nursed, and nurtured by those who recognize their power of future development and application. Thus has the structure of the arts grown by those who have loved them. Medicine stands out as a fitting monument of the devotion of those who have, piece by piece, laid its foundation, and whose foundations are still being added to and strengthened. This structure of learning has become a beautiful temple reared thereon, with its interior brilliant with the records of unselfish lives of the highest type, whose only inspiration could have been love of their fellow-men and the medical art. Tenacity of purpose has thus found the means of healing their infirmities. The rate of progress has been forced by the wide-world cry for alleviation of suffering. Then, again, the development of medical knowledge demands so much from its demonstrators that it necessitates specialization for one man adequately to understand a part and to apply up-to-date and intelligent treatment. But with dentistry the two factors already outlined have been almost absent. Not many students would be drawn by the fascination of success easily gained in its practice, certainly not by the demands on one's physical energies, or by the ever-recurring degree of uncertainty accompanying the use of many materials employed. 


\section{Physical Deterioration has caused a Widespread Demand for Dental Aid.}

Again, the demand for dentistry did not awaken the ingenuity of those who would have readily responded to the call for alleviation of suffering. This is shown within our own time, for of all the various families constituting present-day clienteles, our attention is exercised much more for the children than the parents, and this can be seen for three generations, so we are presumably safe in concluding from the slow progress of dental art and the deterioration of modern generations, that it has not been nursed by favourable circumstances. When we think of our own experiences we almost say that the world has been fortunately watched over by Providence and spared this great bugbear of dental problems.

However, we are, and must be, deeply indebted to those who have practised dentistry before us for the stern determination and patient investigation that have ploughed up a wide field for all who enter to work with equal fortitude; for progress in dentistry still means battling with myriads of difficulties of the same types as formerly, but harassed with conditions that demand some kind of amelioration both for the dental operator and also for the patient.

Physical deterioration has made its effects felt, and among them the deplorable condition of the masticatory organs of the civilized nations. An anomalous state of things exists in our midst, for while in most things the poor do without, or with a meagre supply, yet in dental matters they stand deepest in need, for, being fed in infancy with an insufficiency of bone-forming nourishment, they are more often than not fed afterwards with the very things that cause destruction of their teeth. The subsequent development of their frames are hindered and menaced by the long list of constitutional troubles that are recorded in medical and dental literature, and which are culled usually from clinical practice. Poor children are often left to themselves, and are dependent on what they can get and their parents can provide. Bread forms the staple article of their dietary, which for some years has not been considered satisfactory from a dental point of view.

The dental operator, too, has conditions that harass hin1. The physical strain is great; into a daily habit of more or less 
contortion, the nerves leading from his spine must be interfered with, if only from lack of bodily swing in a normal position of the muscles; the stomach is strained and the posing of the shoulders and arms in using cutting instruments is a definite source of feelings of fatigue after eight hours daily of such work. If conservative work is to become popular the methods of executing it must be made simple and the nature of materials well understood.

Osteo fillings are easily applied, but are not permanent; moreover, the principles of cavity preparation must be carried out even for this, which involves care to prevent burs running hot, to avoid laceration of the gum, and to steer clear of penetration of the horns of the dental pulp, and it all takes time. The more advanced and supposed improved methods take more time, and are only to be judged by the resistance of the substance of the tooth against its destructive factors in the oral cavity.

The man who becomes a dentist very soon finds out that details are his daily portion, and the enquiry of cause and effect taxes his mental energies to a degree that brings him to the realization that attention to details in his methods is absolutely fundamental to the ordinary degree of successful practice. Attention to little things cannot be ignored or made light of or treated as things that are unbecoming to a dignified profession. We must go into the why and wherefore of innumerable points of procedure, otherwise failure dogs our every movement. The dental practitioner must be a philosopher certainly, in order to make his life as tolerable as possible; but for his actual work he must be prepared to cultivate the intuition of a chemist, a natural philosopher, a metallurgist, an artist, an engineer, a surgeon, and a keen, inscrutable observer of all that passes before him, a veritable ferret for unearthing all knowledge that would bring ways and means to the betterment of the execution of his daily work.

\section{The Study of Mechanical Forces and their Application.}

The study of mechanics involves two great laws of force and equilibrium. We naturally recognize and utilize the forces of heat, water, and air, scientifically known as caloric, hydraulic, and pneumatic. We are daily using these three forces in innumerable ways: heat for boiling water, soldering purposes; superheated steam for vulcanizing and casting purposes; air for the blowpipe, Bunsen burner, and casting. These three forces are so involved in our mechanical processes that for the sake of clear- 
ness we will use the terms "dynamic force" and "static equulibrium" as being the primary principles running through the various steps of dental work.

Force and equilibrium are the factors that engage engineers in their great calculations for vast works; but whether a thing is gigantic in size or tiny in proportions, if the same principles are present then the principles must be mastered equally in both cases, and we readily agree that if our work were of larger proportions we should get more credit for the amount of detail it demands.

\section{Definition of Primary States of Matter.}

These two primary states of all matter, dynamic force and static equilibrium, can be interpreted into simpler language as energy and rest. Energy is impelling, rest is sudden and abrupt or rhythmic and gentle, according to the impulse that roused or stimulated the energy. An explosion would cause a rude awakening of the molecules of matter, so much so that the energy suddenly excited in them would cause an abrupt destructive equilibrium. The masses of molecules would become violently rent asunder, steel would be broken into fragments, water driven into spray, and masonry into dust. In this way latent dynamic force suddenly applied means destruction of the molecular mass of matter.

All material substances are made up of atoms. An atom cannot exist in a separate state, but unites with one or more atoms to form a molecule. The atoms of different elements have definite relative weights, and invariable for each, the weight of an atom of hydrogen being regarded as the standard of unity. The actual weight of an atom is not known, but atomic weight is the weight of an atom compared with the hydrogen atom. Atoms make up a molecule, molecules make up a mass, a mass makes up the concrete form of matter. An atom is one of the exceedingly minute particles of matter, aggregates of an immense number of which, held in their place by molecular forces, constitute all material bodies.

\section{Degree of Heat a Condition of Equilibrium.}

The atoms of the various elements that make up a solid body, be they gaseous, solid, or liquid, have a degree of heat which is a constant quantity. The disturbance of this normal heat produces agitation of the atoms, whereby they are not in their 
normal position in relation to one another. They either are repelled or attracted, causing contraction or expansion. Molecular forces consist of certain attractions and repulsions which keep molecules of matter together without touching each other. Molecular attraction tends to draw together the atoms or mole. cules of the same body. It is only exerted at infinitely small distances and produces cohesion, affinity, and adhesion. These molecular forces apply to all bodies whether they are solid, liquid, or gaseous.

\section{The Control of Dynamic Force and Static Equilibrium is the Adjustment that makes for Harmony universally.}

The control of dynamic force and static equilibrium underlies everything throughout the cosmic universe. Worlds are flung into space and find their equilibrium, travelling in defined orbits, returning to relative points in the heavens, so that astronomers can calculate within a few minutes their reappearance at these points. The seasons on this planet afford us a gigantic display of the endless variety of the results of these forces. Climatic conditions stimulate chemical energy, which involves the displacement of molecules. Storms may be destructive, but violent energy is Nature's effort to regain her equilibrium. When that is secured, the balance of molecular forces does its work in so beautiful a manner that food, air, and solar benefits form a rich harvest for the benefit of mankind.

Again, these controlled forces secure to our benefit the minerals contained in the earth. Chemistry, with its vast resources of the earth's secrets; engineering, with its endless marvels of triumph; architecture, with its monuments of consummate beauty-these all demonstrate the abundance of evidence that matter is under natural or applied control. Further than this, the same control of these forces is applied to all the details of daily life-social, domestic, and technical.

In our social intercourse, public or private, we must have control of our physical forces or we become uncouth. There must always be the balance of relation of force to rest; if not, life would be intolerable in our dealings one with another. Shouting would jar the delicate mechanism of the ear. Rushing about regardless of other people or things would bring calamity upon our own persons or others, and accidents would be general. A 
disregard for order would lead to chaos, and life for every living thing would be problematical. In the domestic departments of life a very happy combination of the utilization of control is demonstrated. Although unconsciously, as a general rule, cooks utilize the energies of physical laws and chemical substances to produce an endless variety of dishes, the ingenuity of utilizing these forces furnishes us with an example of the delicate control of applied force and resultant equilibrium, otherwise the lack of control would enforce itself upon us in many disagreeable ways: steam for nearly everything; water in measured quantities, to produce certain results; the coagulating qualities of certain substances, the crystallization of others; gases, too, utilized to produce extreme porosity or lightness in some things, or an icy condition in others.

Throughout the technical world our subject is demonstrated in a practical and convincing way. The more skilled a workman may become in any trade, vocation, or art, it is brought about by his consciously or unconsciously becoming a complete master of his implements, tools, or instruments. If he be a gravedigger, the application of his spade to the ground he is digging will indicate his knowledge of the nature of the materials he is working on, and the capability of force in his spade, shows that the man is marked out for work with more skill in it. The bricklayer lays his bricks with a touch that only a skilled man can apply, so much so that the bricks fall into their place on to the mortar with resilience, securing perfect adhesion and the greatest strength to the wall as a whole. His work is not automatic, as it might appear; the bricks are porous and the cement is wet, and the first shot secures perfection; adhesion is secured with the water of the cement carrying the crystallization into the porous holes of the bricks.

\section{An Efficient Illustration of Control.}

The musician who becomes skilled only in the mechanical use of his instrument may be a delight to himself but not to other people, for the delight that carries the hearer out of himself and transforms the terrestrial atmosphere into a celestial paradise is magically brought about by the subtlety of the performer who in the inspiration for his art finds expression in the control he demonstrates by his touch, which means that he sets going 
sufficient measured dynamic force to secure equilibrium at a definite moment: more or less of this energy would mean failure, there would be no accent, no rhythm, no resonance, no sweetness, no rests, and no soul in the music.

The painter would daub and blotch his picture into unmeaningness; but in proportion to his control of the brushes and his knowledge of the colours, so his picture would become endowed with a lifelike reality. The surgeon surely is master of control of these physical forces in the application of his dangerous instruments which have for their purpose the successful application for certain procedures. The highly complex mechanism of the human organism must be delicately interfered with; too much by a hair's breadth may mean dire consequences. Generally speaking, a surgeon's work is capable of the highest interpretation of the benefits of applied forces to secure definite results.

Artistic skill can be well summed up by the lines contained in Homer's immortal "Iliad " (Pope, book xxiii., pp. 383-394):

It is not by strength, but art, obtains the prize,

And to be swift is less than to be wise,

'Tis more by art than force of numerous strokes

The dexterous woodman shakes the stubborn oaks:

By art the pilot, through the boiling deep

And howling tempest, steers the fearless ship;

And 'tis the artist wins the glorious colirse,

Not those who trust in chariots and in horse.

In vain, unskilful to the goal they strive,

And short or wide the ungoverned course drive,

While with sure skill, though with inferior steeds

The knowing racer to his end proceeds.

The artist in any craft is one who seems to have an intuitive charm over his tools and materials, whereby the work seems imperceptibly and with consummate ease to come intc being, whereas the unskilled man seems to make his work laborious, and everybody seems distressed by the noise and fuss that is made to produce the same class of work.

This outline of the universal working of the great laws of force and equilibrium has been prepared in order that the outlined relations of these forces to the atomic, molecular, and massive structure of all matter may lead to the application of these same laws and relative structure of matter to the underlying principles of practical dental mechanics and be productive of thoughtfulness in carrying them out. The materials used are mostly of a highlv 
technical manufacture, and advanced processes are constantly being called into operation, so much so that a new process cannot be adequately or fairly judged if the treatment of materials is not properly utilized. Again, thoughtfulness means working quickly and successfully. Rushing on without time to think means a muddle and disorder which results in characteristic chaos. In dentistry, where one man is called upon to use intuitions belonging to several other callings, his work stands out as a record of the intelligent and faithful application of the laws of dynamic force and static equilibrium.

\section{Technics of an Art to be derived from an Abundance of Sources.}

Technical knowledge, above all other classes of literary topics. is capable of endless description, and the market statement that books are out of date in ten years is especially true in this class of knowledge. Therefore these books require constant vigilance in order to prepare succeeding up-to-date editions of any particular work.

A student goes up for a course of lectures, and, if of a conversational temperament, will probably glean as much from his fellow-students as he does from the lectures, not because the lectures are without a full source of information, but because he hears the subject in other forms and applied under various conditions, so that the lectures, coupled with social intercourse become a real benefit to him. Again, the student who takes advantage of the allied subjects and even irrelevant ones, will always find an abundant store of information which, if held in suspension in his mind, will at some time or other stand him in good stead in helping him to stand upon his own feet even when he becomes quite competent in his craft. Independence and originality of thought produces a competent man.

\section{Reading is Useless unless assimilated through Practice.}

Textbooks, of course, are most essential to the student in forming his ideas. Ideas must be based upon knowledge. But knowledge is of the greatest value when it is actually demonstrated, and in many cases textbooks are so complete and overflowing with description that a student often complains that 
the textbook is of no use to him because he is compelled to wade through a weary flood of what he may never see again or even require, and therefore becomes discouraged in his search for information. He often finds that the pathway to knowledge is closed by the technicalities which exist in the books, or by the pedantry apparently contained therein. Books also presuppose previous knowledge, which leads to failure to comprehend the information needed.

\section{The Great Value of Textbooks.}

Textbooks are, however, the authorities to which the experienced man often turns with confidence.and pleasure, finding that the broad principles are really well laid down and defined. The student groping with lack of confidence, eager to find a specific instance detailed, is discouraged because he cannot find his particular case described.

\section{Assimilated Knowledge versus Cramming.}

The value of a standardized curriculum cannot be overrated, but its true value to the public is in the amount of that standardized learning being an integral part of the student's mental organization. A degree or a diploma certainly is a guarantee of the opportunity of acquiring knowledge, but is no guarantee of the amount of technical knowledge really gained. The converse may be true in many cases - that the absence of a diploma does not necessarily imply lack of knowledge-for with the opportunities afforded at the present time with clinical experience in denta] practice, coupled with the study of invaluable literary works on medicine, chemistry, dentistry, mechanics, and a liberal number of associated arts, coupled with the fascinating use and application of beautifully-manufactured dental and scientific instruments, it is within the bounds of possibility to acquire an amount of technical knowledge that would not be represented by some diplomas. It is, therefore, the bounden duty of every dental practitioner to gain practical knowledge wherever he can, to hunger for that information that will enable him to do better work. Books and written articles are not of such great value unless their contents are submitted to practical and tangible analysis by experiment first, and then sorting out what is required by the particular reader. 


\section{The Acquisition of Dental Knowledge depends upon a Ruminative Habit.}

Dental knowledge, like all other arts, is derived from various channels of information, from books, better still from experienced clinical practice, personal conversation with practical men, intelligent observation of other arts so as to annex what may be suitable, and, above all, by the use of common sense. So that all kinds of methods, materials, and objects may be transferred to possible use in one's own sphere. It is the recorded opinion of Samuel Johnson that " he who enlarges his curiosity after the works of Nature demonstrably multiplies the inlets to his own happiness." This axiom can certainly be applied to the gaining of dental knowledge. Dental science is ever advancing, and we have to keep abreast of the times. Journals, articles, catalognes and even circulars describing new instruments and materials will help, but these must be submitted to the focus of our mental vision; they must be scen as well as read; mental perception as well as impression must take place. It has been said that there is a close connection between Nature and invention, and that there is scarcely an invention of man that has not its prototype in Nature, and that the greatest results have been obtained from means apparently the most insignificant. We may therefore argue from this statement that all difficulties may be overcome by the man who uses his eyes and brains to a sufficient degree.

\section{The Dental Practitioner must be essentially a Self-reliant Man. The Comprehension of Principles is the Key that unlocks Difficulties.}

But while this assumption is impossible to one man, owing to his limitations of time and energy, yet nevertheless the dentist is a man who must combat difficulties often at a moment's notice, and must find a way of dealing with them by relying upon his own powers of originality and ingenuity. If he understands the principles of his work he already holds the key to difficulties that will arise as well as to those that do not come across his path, while to be a slave of this difficulty or the other would take a lifetime and more in order to equip him for his work. The comprehension of these principles must obviously make it easier for him to carry out his work, not only successfully, but quickly; 
therefore time and study spent in getting at the purpose or object of any method or process should enable. a man to do his work expeditiously and in a masterly manner. Our byword in these chapters will be: What is the object or purpose underlying what we are doing at every point? This is another way of expressing what the principle of an art means.

In this way, if a dozen men were treating a similar tooth and an uninitiated man stood watching them, he might be perplexed by the various ways he witnessed the carrying out of the operation, and also by the various explanations these men gave him. But if a competent man were watching he would not be impressed by these different ways of doing the operation; possibly he could show still more ways of doing the same thing. This latter man is therefore not confused, but understands that there is an object or purpose common to them all, and he calls this common object or purpose the principle of the work. He is not the slave of any particular method or materials, but understands the anatomy, chemistry, disease or morbid anatomy, diagnosis, prognosis, and the complete restoration of the part he is operating upon. He is in a position to use anything that lends itself tc the obedience of the principles he has to apply. 


\section{CHAPTER II}

\section{A DESCRIPTION OF EMBRYONIC DENTITION}

As operative dentistry may be considered as a branch of the healing art, therefore we may say that it is of a reparable nature. In other words, our operating is applied in order to repair or make good that part of the mouth which it is our department or province to attend to. Our work is of a surgical nature, yet it is largely mechanical, and involves engineering principles on a tiny scale, in order to restore lost parts of the teeth to render them useful again in mastication. Therefore we are in a similar position to a man who repairs machinery of a specialized nature. In the engineering world it would not be fair to the owner of a highly complicated engine if it were to be repaired by a mechanic, however clever or skilful he might be in his craftsmanship, because he might, unwittingly, injure some material or part which might ultimately impair the capacity of the engine. Therefore, for the repairs to be properly and fairly executed it would require the services of the engineer who planned the building and making of the machine and who knew the exact nature of the functions and parts of that machine, in order that their integrity might be preserved. No one would take a costly motor-car or a beautifully balanced watch to anybody who claimed to do repairs neatly, but they would jealously consult someone who had a comprehensive and yet practical knowledge of these machines before any repairs were undertaken.

Now we must admit that we have to work upon the most intricate, highly organized, and beautifully balanced mechanism that exists in this universe. In order to do justice to the patient we must not only have a knowledge of the working of the human machine before proceeding with our manipulative work, but a conception and comprehension that will make the knowledge of it as real and familiar to us as the acquaintance we have with our mechanical methods for our restorative work.

We have to employ methods of restoring lost parts of teeth, which we term " filling of cavities, inlay work, and crown work"; 
we may also utilize and apply mechanical devices and use physical force to move teeth which are irregularly placed, which we call "regulation," or we may have to remove diseased teeth from their sockets, which we designate " extraction." We must precede all these various branches of operating by an ever-searching investigation into the nature and construction of the teeth and their sockets. We will proceed to make a clear but brief outline of these extensive subjects as they directly affect us.

In order that our examination may lead us to an intelligent opinion as a basis for operative treatment we must understand the processes of development of-

\section{Dentition, Gum, and Sockets.}

Dentition is the process by which the teeth, or, more properly speaking, the parts of the teeth, develop from their origin and then from their bony crypts upwards to the surface of the moutl, which we call the ridge.

Before going any further we will state that the teeth have their origin in the mucous membrane of the mouth, and after a very interesting process of evolution and development in the deep tissues of the jaws they finally take their place in the parts from which they started.

The nearest physiological process in the body is the emergence of the hair sheaths and nails from their germ cells to eruption.

We will take certain ages of the child with a view of making the process clear, so that the practitioner will be able to define in his own mind the probable state of the dentition at their approximal ages. Dentition may be normal or abnormal. If abnormal, pain and serious systemic conditions often accompany it. It is a physiological process; that is to say, it is capable of being described histologically, anatomically, and functionally.

Anatomy and physiology are subjects that are bound up with each other, but the subjects are so vast that it is more practical to deal with them as separate subjects. But anatomy deals with the description of structures as they are, while physiology investigates their functions. Physiological anatomy examines structures with relation to function, but physiology demonstrates anatomical and chemical functions. Histology examines and treats of the minute structure of the component tissues of the body. The structures and organs of the body are themselves 
built up of tissues which can only be studied by the aid of the microscope. Embryology embraces these three subjects when applied to the origin and development of tissues and organs before birth and which aggregate and group to form the fœtus.

Anatomists, until recent years, have looked upon the teeth as being members of the osseous or bony fragments of the body, but they are now placed in the dermal class of tissues. Microscopic research for many years has been so wonderful that examination of the dental tissues has been investigated with definite success, so that the development of the teeth from their earliest signs of evidence can be traced, and are fully understood by modern histologists and anatomists. Their investigations are materially assisted and proven by careful comparison with the same organs found in other animals. This co-study is called comparative anatomy. The results of these investigations have proved that the enamel-forming organs have their origin in the epithelial tissues. The epithelial tissues are the surfaces of the mucous membrane, and also of the skin of the body. It is of a cellular structure. The enamel-forming organs are formed therefore of the same tissue elements as the hair, the nails, and the outer linings of the glandular structures of the skin and the mucous membrane, namely, epithelial cells.

A cell is the minutest part of matter capable of the manifestation of life. It is a structural element which, under favourable conditions, is able to nourish itself, develop, and produce other cells. It consists of a semi-fluid, albuminous mass called protoplasm, and a more solid mass floating in the semi-fluid, which is called a nucleus. This nucleus of the cell is a minute vesicular body generally located near the centre of the cell; it is round, oval, or irregular in shape, and one or more in number. Its function is to reproduce the cell, and is essential to the growth and nutrition of the protoplasm.

Protoplasm consists of a soft, greatly distensible semi-fluid substance, which forms the greater part of the cell, so that the essential parts of a cell are the protoplasm and the nucleus. The cells multiply and continue formation and development by a process called proliferation. Protoplasm is the living matter from which all kinds of living beings are formed and developed, and to the properties of which all their functions are ultimately. or originally referred. It is a transparent, homogeneous, or granular-looking substance. Water, dilute acids, and alkalis 
cause protoplasm to swell up and ultimately become disintegrated; and it is coagulated by those substances or chemicals that coagulate proteids or albumen. It is now known that in the embryo and adult of both plants and animals, vertebrates and invertebrates, all kinds of cells, before their protoplasm undergoes division, show complicated change of their nucleus which leads to division and separation into fresh cells. The cell, again, is a term applied to a microscopical anatomical element with a nucleus cell wall and cell contents when typically or perfectly formed. The animal cell is ordinarily a closed sac, the outer or environing membrane always consisting of a nitrogenous compound. This sac contains the semi-liquid called protoplasm, and in which are suspended molecules, granules, globules, and other very minute cells.

In order to form some idea of the development of the enamel organ it will be as well to briefly describe the character of epithelial tissue. In the earliest stages of the embryonic life of animals which are developed from an ovum there occurs a rapid proliferation or multiplying of embryonic cells in the germinal spot of the ovum called the disc of Pander. This disc is at first composed of a germinal cell, which by a certain process produces a rapid multiplication of these elementary or embryonic cells.

\section{Tenth Day Fœtal Life.}

About the tenth day after pregnation it forms a small body called the blastoderm or dermoblast. This body then begins to arrange itself into three layers of cells:

The Epiderm or Epiblast.

The Hypoderm or Hypoblast.

The Mesoderm or Mesoblast.

From these three layers of cells, all of the tissues and the various organs of the body are developed. From the epiblast layer of cells are developed the various layers of the skin-viz., the epidermis or true skin, also the glandular appendages of the skin, the hair, the nails, and the entire nervous system.

From the hypoblast layer of cells are developed the lining mucous membrane of the alimentary tract or digestive canal, of the air passages, of the genital apparatus, the epithelial linings of the serous cavities, such as the linings of the bloodvessels and 
the glands of the body, the lining membrane of the heart, the blood, lymphatic vessels, and the enamel organs of the teeth.

These two layers are formed first, and then from the mesoblast, which is the third layer, appearing between the epiblast and the hypoblast, are developed the remaining portions of the bodyviz., the bones, muscles, bloodvessels, lymphatic vessels, and connective tissues.

It will be noticed that the first layer of cells contains the germs of the skin or outer covering of the body. The third layer of cells contains the germs of the bones, muscles, bloodvessels, etc. The second layer of cells contains the germs of the mucous membrane, . which is the skin or covering of the oral cavity, the digestive tract, and the inner cavities of the body.

The two layers of cells that are first formed contain the germs that will develop the external skin and the internal skin or mucous membrane. The third layer of cells contains the germs of the great structures such as the vascular, muscular, and skeletal systems, the generative glands, and the kidneys. So that it will be seen that the external and internal coverings with their germs are set going first, and then the germs of the great internal apparatus are developed within the first two a little later on.

The cells are of varied shapes according to what part they ultimately develop.

The epithelial cells are common to both the skin and mucous membrane, their peculiar characteristics depending upon their position or location and their particular function. They vary in shape and are:

Squamous or scaly.

Cuboidal.

Columnar.

Spinous or furrowed.

Ciliated.

These various-shaped cells form the various parts of the hair sheaths, hair shafts, also the nails or horny layers.

In the mucous membrane of the mouth the top layer of epithelial cells are of the squamous or scaly variety, while immediately beneath this layer lies a layer of spinous or furrowed cells, the spines of which interlock with neighbouring cells. Beneath this is a third layer of cells made up of small soft roundish cells, sometimes oval, and arranged in a regular order, standing upright 
upon the basement membrane. This is called the Malpighian layer. From this layer the enamel-forming organs of the teeth are formed.

\section{Eighteenth and Twenty-fifth Day of Fœtal Life.}

In the human subject the first evidence of the formation of the upper and lower jaws is seen about the eighteenth day after conception, and consists of parts called processes. They form a good way back and then elongate until they approach each other towards the median or middle line, where they finally join or coalesce at about the twenty-fifth day to form the superior maxillary and inferior maxillary bones termed the upper and lower jaws.

\section{Eighteenth Day.}

The upper jaw is evolved at about the eighteenth day after conception by the development of four tiny buds called tubercles or processes near the centre of the region of what will become the face.

\section{Twenty-eighth Day.}

About the same time the lower jaw is evolved by the development of two similar buds, which grow rapidly towards the median or central line, where they unite at about the twenty-eighth day, completing the arch of the lower jaw. A short time afterwards a cartilaginous band makes its appearance within the central portion of this rudimentary jaw, called Meckel's cartilage. The function of Meckel's cartilage is to give form and stability to the inferior arch until ossification takes place, when it disappears by absorption, or becomes calcified, forming a part of the submaxillary bone which we call the mandible or lower jaw.

\section{Seventh Week.}

The earliest evidences of the evolution of the teeth are to be found about the seventh week of intra-uterine life in the shape of a depression of the Malpighian layer or stratum of epithelial cells upon the alveolar border of the rudimentary jaws forming what is known as the epithelial band. This band gradually descends into the embryonic tissues of the jaws, and curves slightly inward and is heaped up with flattened epithelial cells, making what is known as the dental ridge, or the maxillary 
rampart by some anatomists. The next step towards the development of the future tooth is when this epithelial band sends off a thin lamina or process upon the lingual side called the epithelial lamina. After this, small buds or processes appear upon the extremity or edge of the lamina called the epithelial cords. They then elongate and burrow down into the embryonic tissue of the jaws. When these cords have penetrated sufficiently deep they enlarge at the ends and become club-shaped.

A

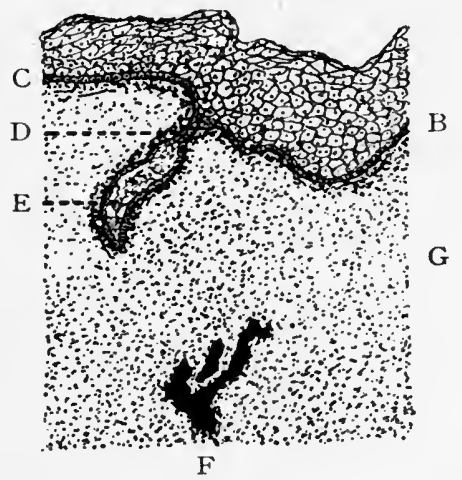

Fig. I.-Section through Lower Jaw of Foetal Pig.

(Sewill's " Dental Surgery.")

This preparation corresponds with the phase of dental evolution existing in the human embryo at about the eighth week. (From a preparation by Mr. Hopewell Smith.)

A, Oral epithelium; B, deeper layer of epithelium; C, deepest layer of epithelium (rete Malpighii); D. Primary inflection of enamel organ (primitive tooth band); E. internal epithelium of enamel organ; $\mathrm{F}$, bone of jaw; G, sub-mucous tissue.

\section{Eighth Week.}

These club-shaped enlargements are clearly defined at the ends of the extremities of the epithelial cord at about the eighth week and are called the enamel organs (Fig. I).

These processes advance at the same time in all the deciduous teeth. The club-like enamel organs now rapidly enlarge and become pear-shaped in form. This pear-shaped enlargement is due to the activity of the cells which now form the outward boundary. The enamel organ has now attained its full size, and occupies a position in the deeper structures of the jaw, and is slightly inclined towards the region of the tongue. 


\section{Ninth Week.}

The next important step in the development of the tooth is what is known as the invagination of the enamel organs. This term means a sheathing of the enamel organs, which occurs simultaneously with the appearance and growth of the dentinal papillæ. As soon as this new organ has assumed a conical form, which occurs about the ninth week, there arises from its base two

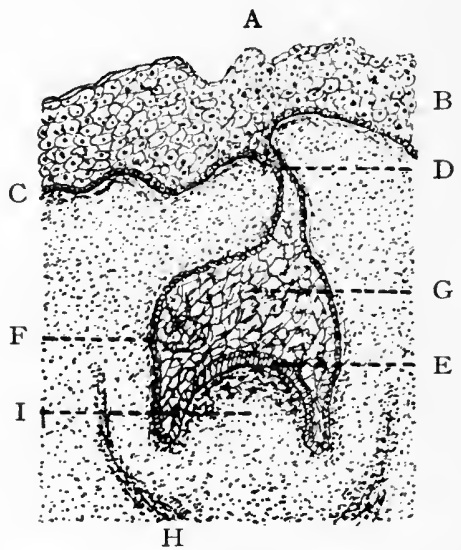

Fig. 2.-Section through Lower Jaw of feetal Pig.

(Sewill's "Dental Surgery.")

This preparation corresponds with the phase of dental evolution existing in the human embryo at about the tenth week. (From a preparation by Mr. Hopewell Smith.)

A, Oral epithelium; B, deeper layer of epithelium; C, deepest layer of epithelium (rete Malpighii); D, neck of enamel organ; E, internal epithelium of enamel organ; $F$, external epithelinm of enamel organ; $G$, stellate reticulum of enamel organ; $H$, commencing formation of dental sac; I, dentine germ.

opaque processes which are the first traces of the wall or sac of the future dental follicle. The wall or sac which surrounds the base of the dentinal papillæ like a collar elongates as the dentine bulb, enclosing the dentinal papillæ, increases in size until it finally surrounds the dentine bulb and the enamel organ. As the dentine bulb enlarges and elongates it comes in contact with the lower surface of the enamel organ, which is then carried upward until it is completely ensheathed, thus forming a cap or hood over the dentinal papillæ. There is now a reciprocal adaptation of the 
enamel organ to the dentinal papillæ which continues throughout all subsequent phases, the enamel organ at all times covering the dentinal papillæ and adapting itself to its varying contours. It must be understood that at no time during the process of development do the tissues of these two organs become united, but although contiguous are yet separate (Fig. 2).

A

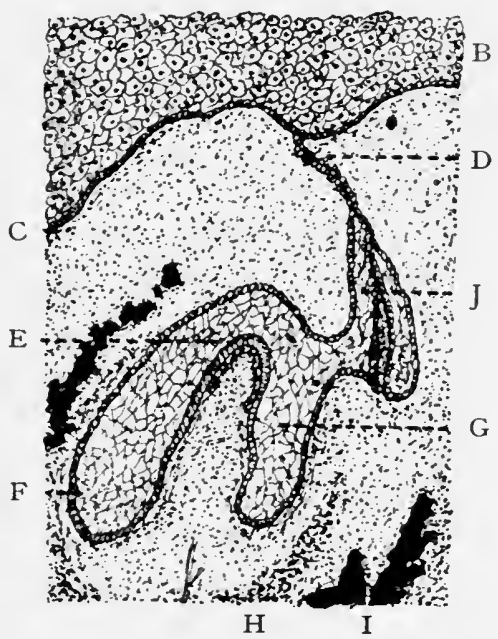

Fig. 3.-Section through Lower Jaw of Fetal Pig.

(Sewill's "Dental Surgery.")

This preparation corresponds with the phase of dental evolution existing in the human embryo at about the thirteenth week. (From a preparation by Mr. Hopewell Smith.)

A, Oral epithelium; B, deeper layers of epithelium; C, rete Malpighii; $D$, neck of enamel organ (remains of primitive tooth band); E, internal epithelium of enamel organ; F, external epithelium of enamel organ; $\mathrm{G}$, stellate reticulum; $\mathrm{H}$, commencing formation of dental sac; $I$, bone of jaw; J, permanent tooth germ.

\section{Eleventh Week.}

At about the eleventh week an important change has taken place in the shape of the dentinal papillæ, which have now assumed the forms of the crowns of the teeth they represent. There is also a narrowing or constriction taking place at the bases of the papillæ, forming a kind of neck at the free border of the enamel organ (Fig. 3). 


\section{Sixteenth Week.}

The next important change in the process of development of the embryonic tooth is seen at about the sixteenth week, when a tiny but well-defined cap of calcified dentine is seen at the tips of the incisors and canines, and about a week later in the molars.

Throughout all the stages we have gone through it must be understood that the enamel organs and dentinal papillæ are in a soft cellular condition, all parts being in a full active condition of proliferation or multiplying cells. At this period we notice calcification of a small portion of dentine in the deciduous teeth mentioned above. Calcification is the term applied to the deposition of calcic or lime salts which form the hard tooth tissue. It will also be noticed that the dentine is the first part to harden or calcify. Calcification has not yet begun in the ameloblasts of the enamel organ, although they are now fully developed and well defined. The epithelial cord is still attached to the enamel organ, but the sac is not yet closed (Fig. 4).

There are now two interesting processes at work, one in the enamel papillæ and the other in the dentinal papillæ.

The papillæ, having been formed within the enamel organ, a beautiful layer of regular, epithelial cells which produce the hard enamel rods are now to be seen fully developed and well defined. Within them appear stellate or star-shaped bodies. The cells of this beautiful layer are called ameloblasts or enamel-producing cells.

The papillæ also having been formed within the dentinal bulbs, there is now a layer of formative dentine producing cells fully developed called odontoblasts. They also constitute the outer layer of the dental pulp, and are columnar in form. They do not enter the dentinal papilla but remain at its base and send into it numerous finely branched processes, which run parallel to each other and which become like so many little channels in the bony substance.

Although the ameloblasts make their appearance first, calcification does not take place in them until after the odontoblasts have formed a cap of calcified dentine upon which the enamel rods are to rest. Under the microscope minute glistening bodies are seen, which indicates the near approach of the process of calcification.

Another very important development takes place about this time. The epithelial cords which penetrated the deep tissues 
and ultimately formed the dental follicles of the deciduous teeth are now seen to have buds appearing upon them on their lingual sides and above them in position-that is to say, between the existing follicle and the Malpighian layer. These buds become

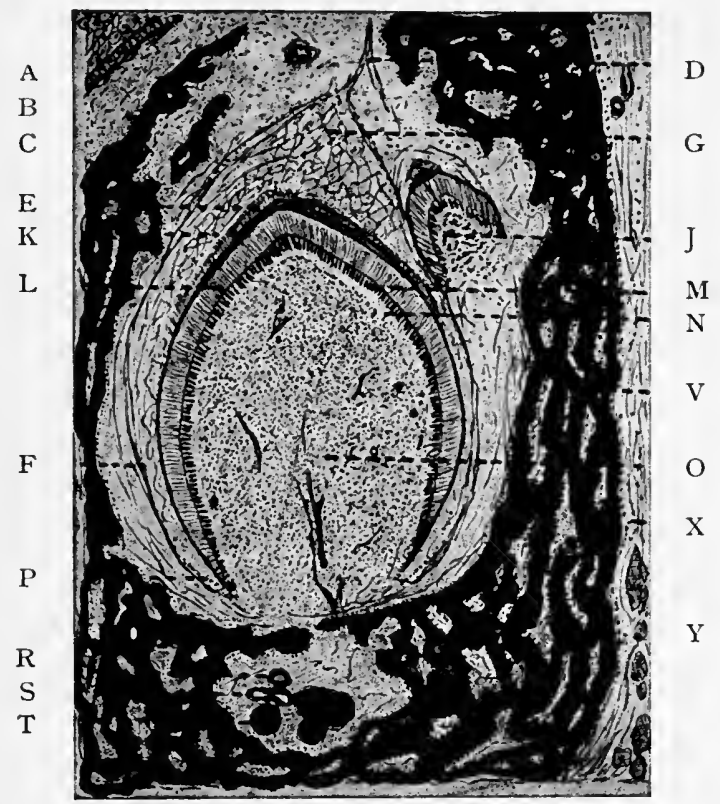

Fig. 4.-Section through the Lower Jaw of a Fetal Pig. (Sewill's “ Dental Surgery.")

This preparation corresponds with the phase of dental evolution existing in the human embryo at about the fourth month. (From a preparation by Mr. Hopewell Smith.)

A, Oral epithelium; B, deeper layer of epithelium; C, deepest layer of epithelium (rete Malpighii); D, neck of enamel organ (primitive tooth band); $E$, internal epithelium of enamel organ and stratum intermedium; F, external epithelium of enamel organ; G, stellate reticulum; J, permanent tooth germ; $\mathrm{K}$, formed enamel; $\mathrm{L}$, formed dentine; $\mathrm{M}$, layer of odontoblasts; $\mathrm{N}$, basal layer of Weil; $\mathrm{O}$, commencing formation of dental pulp; $\mathrm{P}$, epithelial sheath of Hertwig; R, artery; $S$, vein; $T$, nerve fasciculus; $V$, bone of jaw; $X$, muscular fibres; $\mathrm{Y}$, periosteum of alveolus.

definite branches and form epithelial cords to furnish the enamel organs for the ten anterior permanent teeth in each jaw. The buds rapidly assume a pear-shaped form followed by the appearance of the dentinal papillæ as already described for the deciduous teeth. 


\section{Eighteenth or Nineteenth Week.}

At the eighteenth or nineteenth week the sacs of the primitive dental follicles are closed, and the follicles separate from their epithelial cords, and from this time development goes on without connection with the parent epithelial layer. The follicles of the

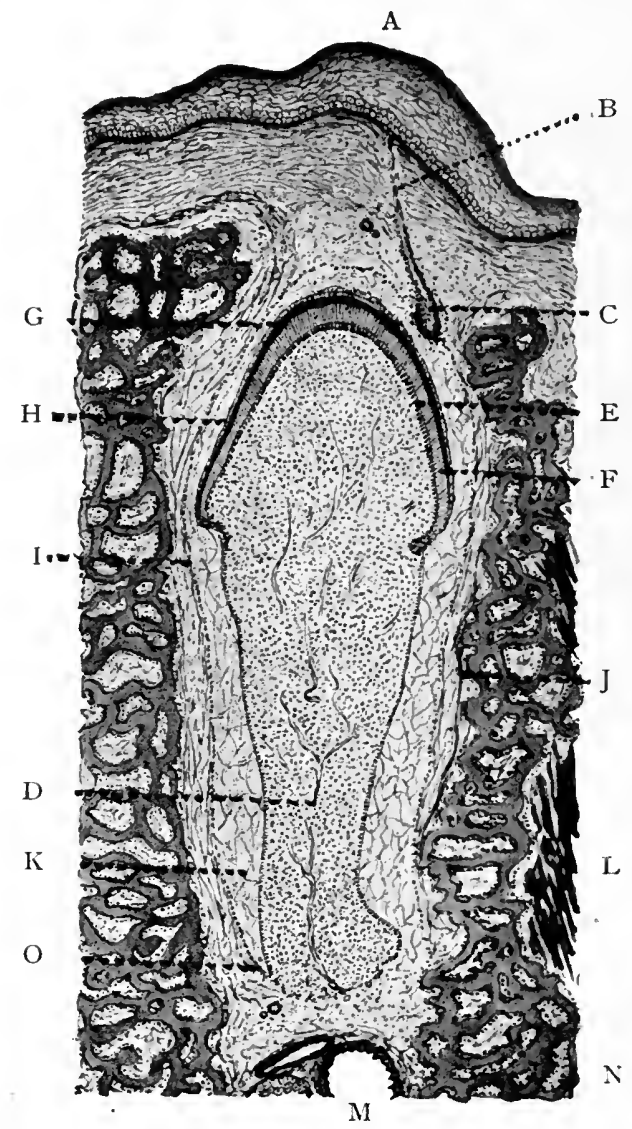

Fig. 5.-Section of Lower Jaw of Human Fetus at about the Fifth Month. (Sewill's "Dental Surgery.")

(From a preparation by Mr. Hopewell Smith.)

A, Oral epithelium; B. neck of tooth germ; C, permanent tooth germ; D, dental pulp; E, odontoblasts; F, dentine; G, enamel; H, ameloblasts and stratum intermedium; I, tooth sac; J, alveolar periosteum ; $K$, stellate reticulum; L, muscular fibres of jaw; $M$, artery, vein, and nerve in transverse section; $\mathrm{N}$, bone of jaw; $\mathrm{O}$, epithelial sheath of Hertwig. 
permanent teeth remain attached to the cord from which they had their origin, and sink deeper into the embryonal tissues, and take their place beneath the follicles of the deciduous teeth, which at a future period they replace.

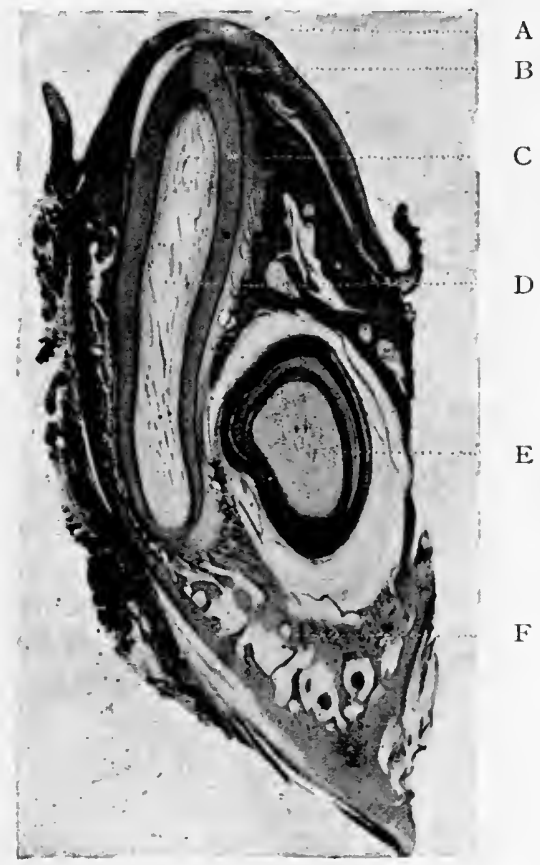

Fig. 6.-Section of Lower Jaw of Foetal Kitten witil Temporary Tooth and Permanent Successor in Situ. $\times 24$ Diameters. (Sewill's "Dental Surgery.")

This preparation corresponds with the phase of evolution in the most developed teeth in the human infant at about the third month after birth. (Photo-micrograph by Mr. Charters White.)

A, Gum; B, enamel; C, dentine; D, dental pulp; E, permanent tooth enclosed in sac; F, jaw partly ossified, and section of inferior dental artery and nerve.

\section{Seventeenth Week.}

The permanent molars do not develop from the epithelial cords of the deciduous teeth. The follicle of the first permanent molar develops from the epithelial cord springing from the posterior end of the Malpighian layer of the mucous membrane, about the seventeenth week, beyond the origin of the cord of the second temporary molar (Fig. 5). 


\section{Second Month after Birth.}

The second permanent molar is developed fiom a branch of the cord of the first permanent molar at about the second or third month after birth (Fig. 6).

\section{Third Year.}

The third permanent molar, or wisdom tooth, is developed from a branch of the cord of the second permanent molar about the third year after birth.

The development of the follicles of the permanent teeth are the same as that described for the deciduous or temporary teeth.

We have endeavoured to describe briefly and clearly the development of the teeth in the embryonic stages of the fotus. It consists of the forming of the epithelial cords penetrating into the deep tissues, then budding at the ends into the enamelforming crgans and the dentine-forming papillæ. Then branches are formed on the sides of the epithelial cords which, in turn, penetrate deeper still, and bud into the enamel and dentineforming papillæ for the twenty permanent teeth, the dental sacs or follicles ultimately becoming detached from the epithelial cords and thereafter development proceeds from the dental pulp. The permanent molars spring from an epithelial cord for the first one, and branches from that to the twelve-year-old and again from that to the wisdom tooth.

This description of the cellular development of the teeth will have prepared us for the consideration of the actual structure of them, as it is imperative that we should thoroughly understand the nature of their normal condition before we can be in a position to undertake the treatment of their morbid condition and their ultimate restoration to usefulness. 


\section{CHAPTER III}

\section{THE STRUCTURAL FORMATION OF THE TEETH}

THE enamel and dentine layers of the tooth crown having been formed from their origin in the epithelium of the mucous membrane, we should now observe that there is a definite change taking place. The crowns have assumed their definite shape, and the layers now consist of beautiful tubular-shaped cells ready for the deposition of the hardening material composed of lime or calcium salts, which will cause the solidifying of the complex structure, and which when completed is apparently, to the uninitiated, a solid tooth. The enamel layer now consists of ameloblasts and the dentinal layer of odontoblasts.

\section{The Existence of Temporary Cellular Organs for the Deposition of Hardening Materials.}

The two layers are formative cells for a specific purpose, and that is, to form the hard enamel rods, and also the inner dentinal tubes. They work by depositing the calcium salts, layer upon layer, and decreasing in size as their depositing work is completed. The ameloblasts and odontoblasts probably become absorbed. The formation of the root is now coming into being, but is the creation of the foster-mother of the tooth-viz., the dental pulp. The follicles or capsules containing the crowns having separated from their epithelial origin at the eighteenth or nineteenth week of fœtal life, the completion of the tooth is now thrown on to the dental pulp.

\section{The Formation of the Tooth transferred to a Foster-Parent.}

The dental pulp, therefore, is a very interesting part of every tooth, as its chief work is the formation of the roots and the completion of the crown. It appears as a streak at the centre of the tissue of the dentine-forming organ, and develops into a large, round, bulbous body as large as the base of the crown. It 
becomes smaller in size, and elongated according to the shape of the completed tooth. Examined under the microscope, there can be seen the matrix or basic substance of the pulp. Its mass is composed largely of undeveloped, soft connective tissues, containing numerous cellular elements of rounded, oval, and spindle forms, and with hairlike processes.

The closing round or invagination of the dental follicle is completed by a surrounding membrane. The cells which stretch out from the base of the dentinal papillæ envelop the sides of the enamel organ, thus forming a complete sac or follicle. This sac at first appears to be an extension of the cellular activity of the dentinal papilla. The cells forming the membrane spread outwards and upwards, gradually enveloping the whole organ. When the follicle is fully developed the sac membrane, called the dental sacculus, consists of two layers, an outer one and an inner one. The inner one contains the cells which form the cementum of the root. It is of a soft and embryonic nature, and rich in cellular elements. The outer membranous layer becomes the peridental membrane or dental periosteum. This consists of a fairly dense fibrous connective-tissue structure. When the tooth is fully formed and finally completed the dental periosteum becomes the means of attachment of the roots of the teeth to their sockets. Additional layers of cementum are added from time to time frcm this peridental membrane, as will be seen in the case of exostosis of the roots of the teeth as the result of longcontinued periostitis. On the other hand, absorption of the roots of the teeth, when the milk teeth are shed, is due to the action of the osteoblasts contained in the dental periosteum.

During the time occupied by the development of the teeth another process has been taking place. The upper and lower jaws have been developing from their original buds or processes (see p. I8). We must now bring this process into line with the completion of the dental follicle, showing how they meet together and develop one with the other, before proceeding with the periods of dentition at various ages of the patient.

The second or permanent teeth which succeed and take the place of the deciduous or temporary ones are called " successional teeth." The permanent molars are called "superadded teeth." The buds for the successional teeth are formed on the original epithelial cords of the temporary teeth, and go through the same processes that we have already described regarding the dental 
follicle. The successional follicles remain attached to the cords from which they had their origin, which were above the deciduous teeth follicles, and then sink deeper into the embryonal tissues, taking their places below the deciduous teeth follicles, which they replace at their due time The cords of the superadded teeth or molars have been described on p. 22.

\section{Origin and Development of the Jaws.}

The fœtal head, about the eighteenth day after conception, has a very large open cavity where the parts of the face ultimately develop. The sides of the head and the region of the orbits present a number of tubercles which develop into the orbits, the nose, and the upper and lower jaws. In the cavity of the mouth, as the soft cellular bones develop, they become invested with deep soft tissues in which the dental follicles are developing, but the follicles have no connection whatever with the developing bones of the jaws in these early stages.

The upper jaw or superior maxilla is seen in the embryo at about the eighteenth day after conception by the development of four tubercles near the central portion of that surface of the rudimentary head which is destined to form the face. These four tubercles are really two pairs, one pair on each side of the head. They are called the superior or frontal processes, or tubercles, and the lateral oblique or maxillary processes. The superior processes elongate downward and approach each other towards the centre of the face, where they finally join about the twenty-fifth day. The lateral processes develop in a similar way, at about the twenty-eighth day, and meet the superior processes, uniting with them to form the lateral halves of the rudimentary superior maxillary bone, palate bones, the cheeks, the lateral portions of the upper lip and the soft palate. The non-completion of the fusion causes the condition known as cleft palate and hare-lip.

At the same period the lower jaw, or mandible, or inferior maxilla, develops from one pair of tiny buds or processes situated in the sides of the head about the position of the lower region of the tongue. They develop forward and meet at the centre of the chin or symphysis, about the twenty-eighth day, thus completing the mandibular arch.

These processes develop a small cartilaginous band which makes 
its appearance about the end of the fourth month in the centre of the thickness of the rudimentary jaw. This is called Meckel's cartilage. The function of Meckel's cartilage is to give stability and form to the mandible until the jaw is fully developed and ossified, when it disappears either by absorption or calcification. This really acts as a strengthener, in a similar way that we put a strengthening wire into a lower wax bite when making a denture to prevent it spreading.

The bones of the body are originated in two ways; one is called intra-membranous and the other intra-cartilaginous. The upper jaw is developed from membrane, but the lower jaw is developed partly from cartilage and partly from membrane.

The dental follicles of the teeth have penetrated the soft tissues in which they have developed, and are in such a position that the dental borders of the rudimentary jaws approximate to them and close up and around them to form the tooth crypts (Fig. 7). These bony crypts completely enclose the follicles, which are round in shape, until their time is due to move towards their places in the dental arch of the surfaces of the jaws. As the movement proceeds the alveoli or sockets are then formed according to the final shape of the completion of the roots.

The stages of the calcification of the roots of every tooth should be noted by every dental operator in dealing with children's teeth, as serious trouble can be caused through not taking these stages into consideration both in filling teeth and in regulating them.

The root of the tooth is completed by the elongation of the pulp, which takes between four and six years to accomplish after the eruption of the crown at the gum margin. Accompanying the very complex changes in the formation of the teeth within the jaw is an equally complex process taking place within the bone itself, and the nature of these changes is of vital importance in considering any operative procedure on children's teeth. A glance at the plate showing a "child's skull" will at once awaken appreciation of this point. The cubic space occupied by the temporary or deciduous teeth, coupled with the crowns of the permanent ones, leave very little actual bony process which must obviously be weakened at every point, especially in the segion of the lower canines and six-year-old molars. The deciduous or temporary teeth are similar in form to the permanent ones, but have slight differences which are characteristic of 
them. They are whitish in colour, and the enamel is more bulbous, and ends somewhat abruptly at the neck. The roots also are more conical and end sharply to a round point as in the canines, or a flattened sharp end as in the centrals and molars.

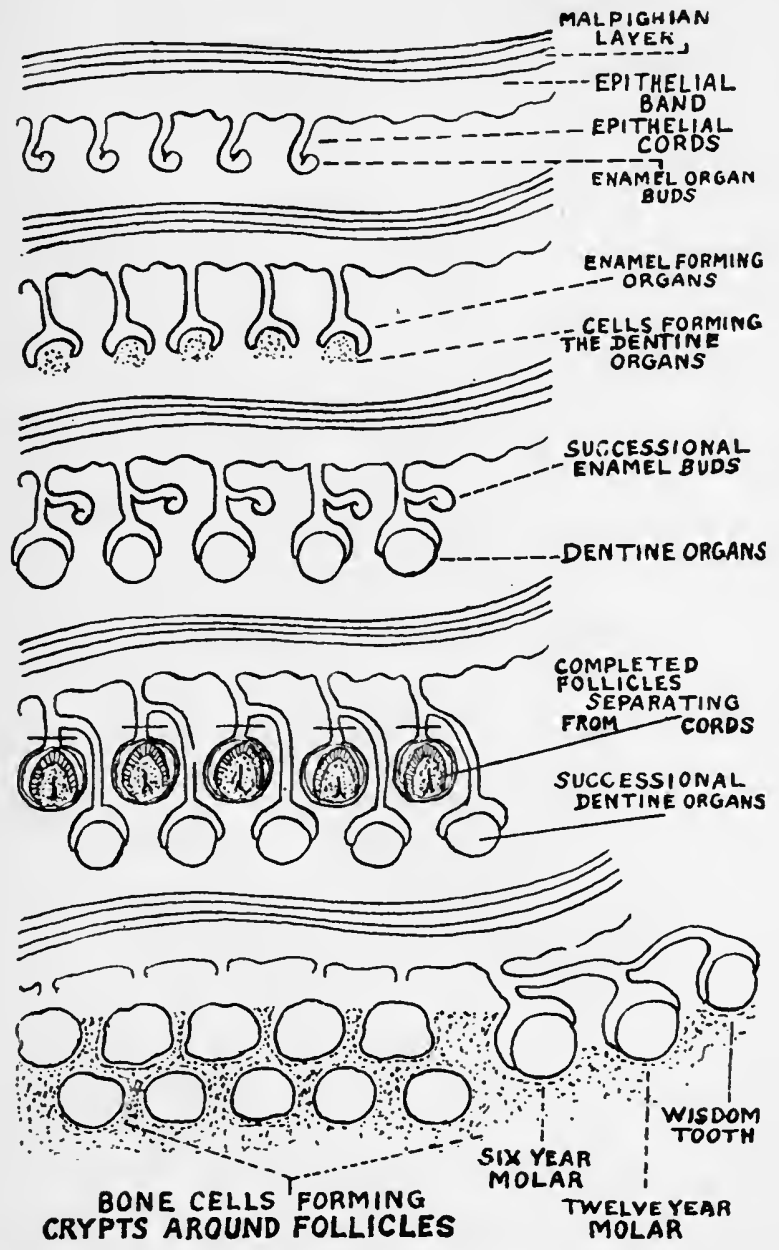

Fig. 7.-Scheme showing Development of Follicles

The process of eruption of the teeth is attended by changes in the bone of the jaws surrounding them (Fig. 8). When the calcification of the tooth tissues is sufficiently advanced to allow of the pressure to which they will be subsequently subjected, the 
process of eruption or upward movement commences. The tooth makes its way through the bone upwards to the gums. The gum is absorbed by the pressure of the crown of the tooth

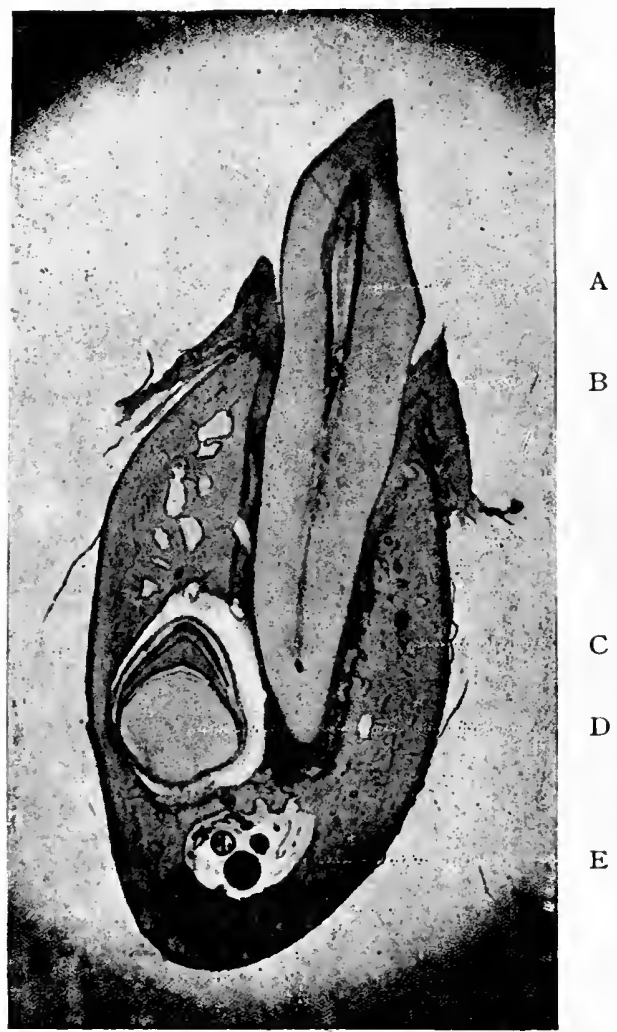

Fici. 8. - Section of Lower Jaw of Kitten, with Temporary Canine Fully Erupted and Permanent Successor in Situ. $x$ Io. (Sewill's "Dental Surgery.")

This preparation displays the conditions immediately after complete eruption of a temporary tooth before absorption of the root has commenced. (Photo-micrograph by Mr. Charters White.)

A, Deciduous canine; $B$, mucous membrane of germ; C, inferior maxilla cartilaginous with islands of forming osseous tissue; D. permanent canine in its sac; $\mathrm{E}$, inferior dental canal, and section of dental artery and nerve.

against it, which is itself pressed up by the increasing size of the fang. At the same time the bony septa or walls of the alveolar sockets between the dental sacs or follicles, which at first are 

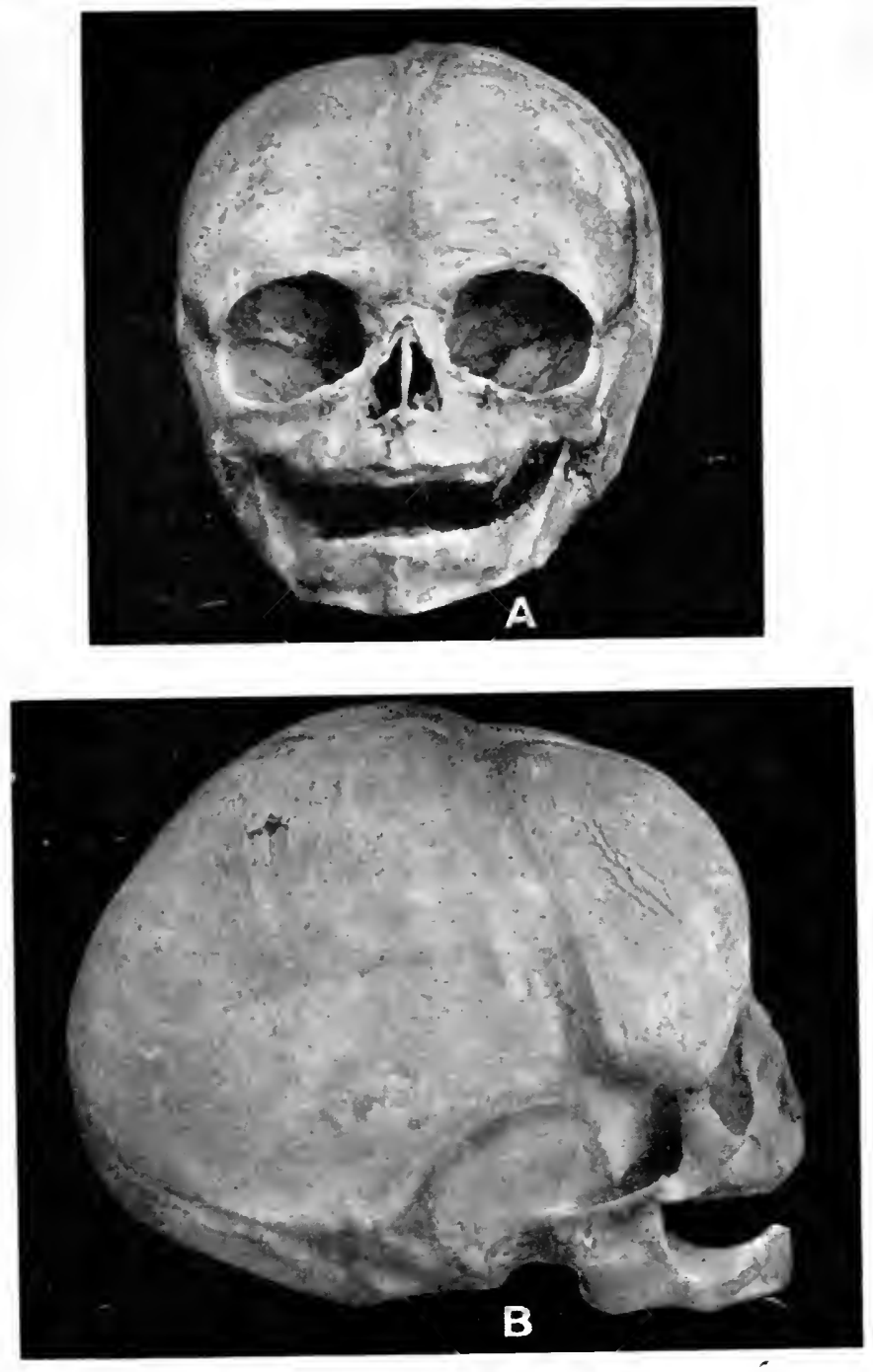

Fig, 9.-Infant's Skull at Birth.

A, Front view; B, side view. 


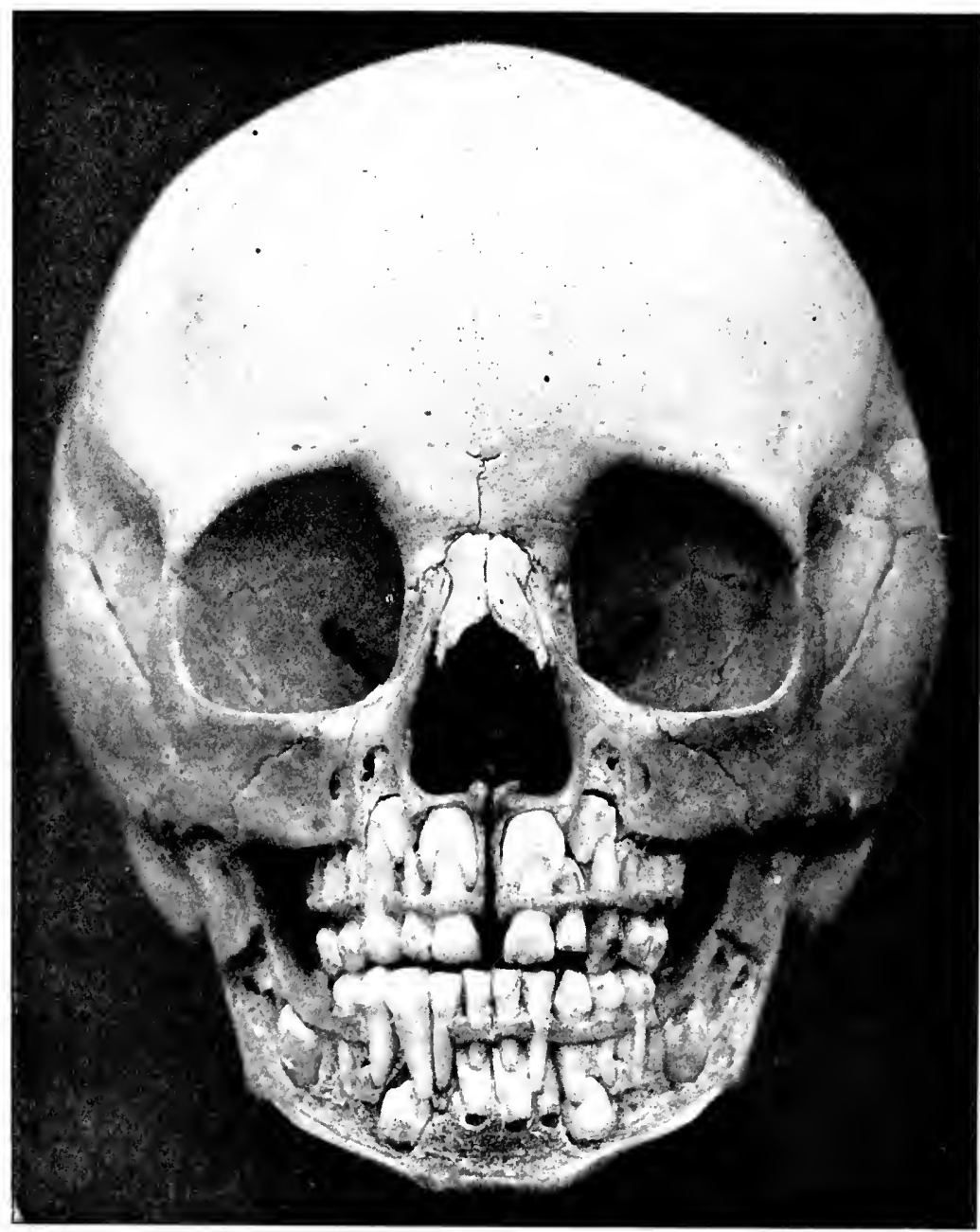

FIG. Io.-ChILD'S SKULL.

Front view. Aged $5 \frac{1}{2}$ years.

Photographed by Mr. Geo. Houlson from a skull in his possession.

(From the Mouth Mirror.)

fibrous in structure, become ossified or calcified and constitute the alveoli. The alveoli in this way become firmly compacted against the necks of the teeth and afford a solid basis of support. 


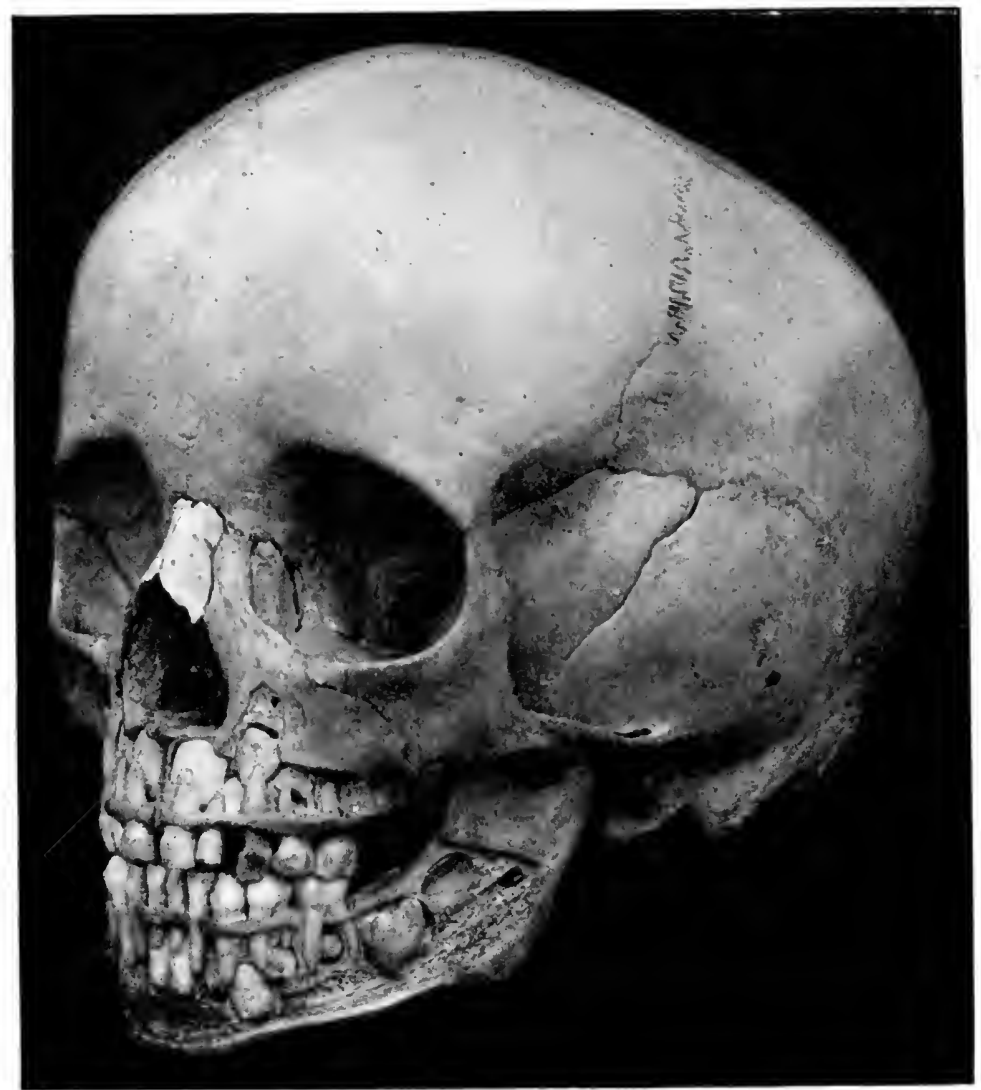

Fig. II.-ChILd'S SkUll.

Side view. Aged $5 \frac{1}{2}$ years.

Photographed by Mr. Geo. Houlson from a skull in his possession. (From the Mouth Mirror.)

\section{Periods of Eruption of Temporary and Permanent Dentition compared.}

The ages of eruption of temporary or deciduous teeth and those of the permanent ones may be seen at a glance by the table on p. 36. This gives the approximate period of the temporary ones and the permanent teeth which replace them. These are termed "successional permanent teeth." The remaining ones are called "superadded permanent teeth" (see p. 28). 


\section{TABLE OF DENTITIONAL ERUPTION.}

TEMPORARY LOWER.

(Ten in number.)
Permanent Lower.

(Sixteen in number.)

Successional.

Years.

6

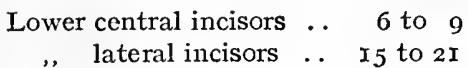

.. I6 to 20

.. I5 to $2 \mathrm{I}$

first molars

second molars

. 20 to 24

Replaced by first bicuspids

Replaced by second bicuspids ..

.. IO

Superadded.

First permanent molars .. 6

Second permanent molars I2

Third or wisdom molars .. I 5 to 30

TEMPORARY UPPER.

(Ten in number.)

Months.

Upper central incisors . . 8 to ro

,. lateral incisors .. 8 to Io

, canines .. .. I6 to 20

" first molars .. I5 to $2 \mathrm{I}$

", second molars .. 20 to 24
Permanent UPPER. (Sixteen in number.)

Successional.

Years.

$\begin{array}{llllll}\text {. } & \ldots & \ldots & \ldots & 7\end{array}$

$\begin{array}{llllll}\text {. } & \ldots & \ldots & \ldots & 7\end{array}$

$\begin{array}{llll}\cdots & \ldots & \ldots & \ldots\end{array}$

Replaced by first bicuspids 9

Replaced by second bicuspids ..

Io

\section{Superadded.}

First permanent molars .. 6

Second permanent molars I2

Third or wisdom molars .. I 5 to 30
TEMPORARY TEETH.

Completion Absorption of Roots. Begins.

Years. Years.
Permanent Teeth.

Completion of Roots.

Years.

$\begin{array}{lll}\text { Centrals } & \ldots & 3 \\ \text { Laterals } & \ldots & 3 \\ \text { Canines } & \ldots & 5 \\ \text { First molars } & \ldots & 4 \\ \text { Second molars } & 5\end{array}$

Centrals ..

Laterals .

Canines .

First bicuspid

Second biscuspids

Six-year molar ..

Twelve-year molar

Wisdom teeth
IO

I I

.. I 3 to $I_{4}$

.. I2

.. I2

.. 9

.. 16

.. 20 to 30 


\section{Symptoms of Normal and Abnormal Dentition.}

Incidents of dentition are numerous, and pathological changes take place which lead to serious systemic conditions, and which sometimes terminate fatally.

In normal dentition there are slight systemic conditions accompanying these changes, such as restlessness, failure of appetite, elevation of temperature of the mouth; and the young child is constantly putting its fingers into its mouth. There is also a constant dribbling of saliva or a superabundance of it in the mouth.

In abnormal cases which may be accelerated or even caused by exposure to cold, and also indigestion, the symptoms of this may be indicated by a hot skin, gums red and swollen, high fever, diarrhœa, eruptions on the skin, and sometimes an ulcerous condition of the tongue, cheeks, and the gums. There is also irritability of temper and great pain, disturbed sleep and wakefulness, great thirst, and oftentimes these conditions lead to convulsions, and if not checked or relieved by a medical man, . end fatally.

Dentition is more favourable in winter than in summer, as the child is not so prone to affections which are produced in hot weather, such as diarrhœa. On the other hand, during winter there is the danger of the child cutting its teeth with bronchitis, croup, pneumonia, or a severe cold. These conditions must be watched and treated by the doctor.

The signs of eruption of the teeth are hot, red, and inflamed gums, increased saliva, a hard tension of the gums, and later a peculiar whiteness caused by the pressure on these tissues by the advancing teeth. Convulsions in a child while teething may even occur in the dental chair, and if there are undoubted signs of an erupting tooth, especially indicated by finding a cusp or cusps sticking through the tightened mucous membrane it may be instantly relieved by lancing the gums over the particular tooth so as to ease the pain by relieving the pressure at the gum surface. The gums are sometimes extraordinarily tough, and lancing is a definite way of giving relief. For front teeth one incision along the cutting edge is sufficient, in canines two cross cuts, and in multicusped teeth a star-shaped incision is suitable. With the eruption of the permanent successional teeth the roots of the temporary or deciduous ones are absorbed by the solvent 
action of a secretion from a fleshy tubercle or layer developed from the sac of the permanent tooth follicle, and is due to the working of certain bodies contained therein called "osteoclasts" or "odontoclasts."

\title{
The Vagaries of Dentition.
}

The normal temporary and permanent dentitions have been described briefly, but there are certain forms of teeth which do not belong to either of these, and are called supernumerary. These are shapeless-looking organs as a rule, but sometimes are duplicates of any of the normal teeth already present. Again, some teeth are joined to their neighbour with a common pulp; these are termed "geminated teeth." Also some teeth, especially molars, are separated as far as the parts of each tooth are concerned, but are joined at the cementum of the roots. Some molars, second ones, have sometimes an elementary-looking peg or tooth attached to them. These incidents of dentition are caused by the vagaries of the epithelial cords, and probably by a crowding of the jaws owing to there being insufficient room for the epithelial cords to bud in or, on the other hand, through an abundance of room in which to develop (Fig. I2).

Having outlined the process of the first and second dentitions leading to their eruption through the gums to their position in the dental arch of the oral cavity, we must proceed to examine the nature of the structure of the tissues within the mouth. We will therefore commence with the teeth themselves and afterwards describe the nature of the neighbouring parts which are frequently involved in any operative procedure in the oral cavity.

\section{Anatomy of the Teeth.}

A tooth consists of four parts:

\author{
The Enamel. \\ The Dentine. \\ The Pulp. \\ The Cementum.
}

The EnAmel is made up of solid rods. They are innumerable in number, and are placed end on to the dentinal layer. They are composed chemically of calcium or lime salts mainly. Various authorities differ in their analyses, for which one can make due 


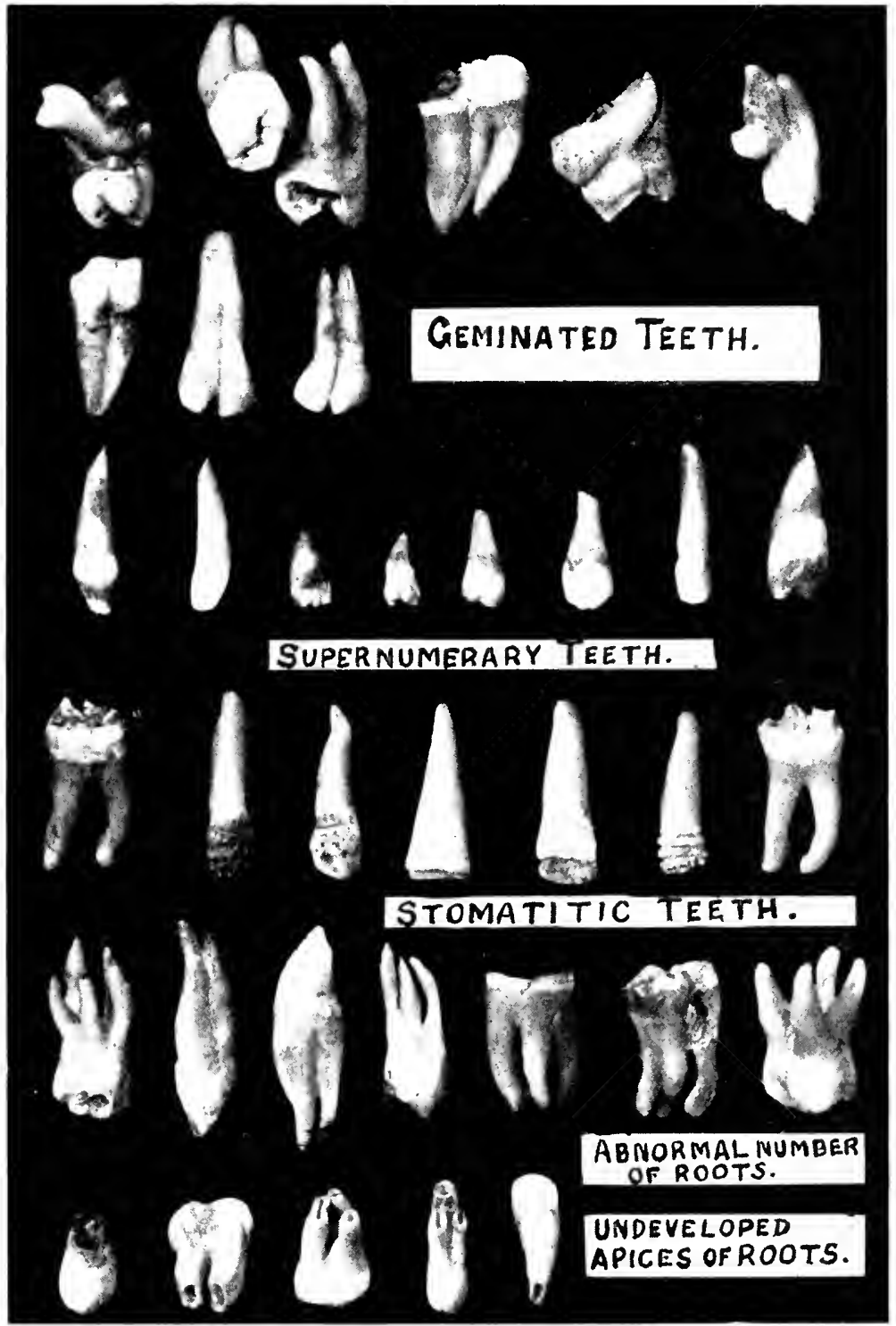

Fig. I2.-Incidents of Dentition. ( $\frac{7}{8}$ natural size.) 
allowances, considering the difficulties of quantitative analysis; but all agree on the materials present. These are:

Calcium carbonate.

Calcium phosphate.

Calcium fluoride.

Magnesium phosphate.

Animal gelatine.

As will be observed by the reader the main constituents are calcium salts and animal matter-calcium to the extent of about 95 per cent. and animal matter about 5 per cent.

The enamel rods are prisms or columns which, while being very fine, are nevertheless separate rods which are capable of being broken down easily when there is a space or cavity underneath them. This apparently hard or adamant tissue, though defying the action of a bur to cut it, can readily be broken down by using an enamel cutter of suitable shape and size. Also cross-cut fissure and flame burs will break them down, but it must be distinctly understood that these burs do not cut the enamel but break it up into particles or even small masses of the rod-like structure. The rods are not of uniform-sized straight shape, but are what is called varicosed or wavy in shape. The spaces between these wavy surfaces are filled in with a weaker calcic material which exposes it to the ravages of bacteria which results in caries of the enamel, and even if this does not take place it will easily be dissolved by acid conditions of the liquids of the mouth (Figs. I3 to I6).

The enamel rods are deposited in layers; these can be detected by the naked eye in some teeth. In others there are marked evidences that the matrix, or mould, in which the calcic deposition took place was not properly filled. This condition is often observed in the mouth by a very irregular surface, and is caused by the inability of the blood to supply the sufficient amount of calcic salts in solution to the dental pulp to enable it to carry out that part of its formative work. An approximate opinion can often be given by the operator as to a probable illness suffered by the patient at a certain age. This is indicated by the irregular surface occurring at certain distances on the surface of the crown.

The Dentine consists of a mass of tubes called " dentinal tubuli." These extend from under the enamel layer right through 

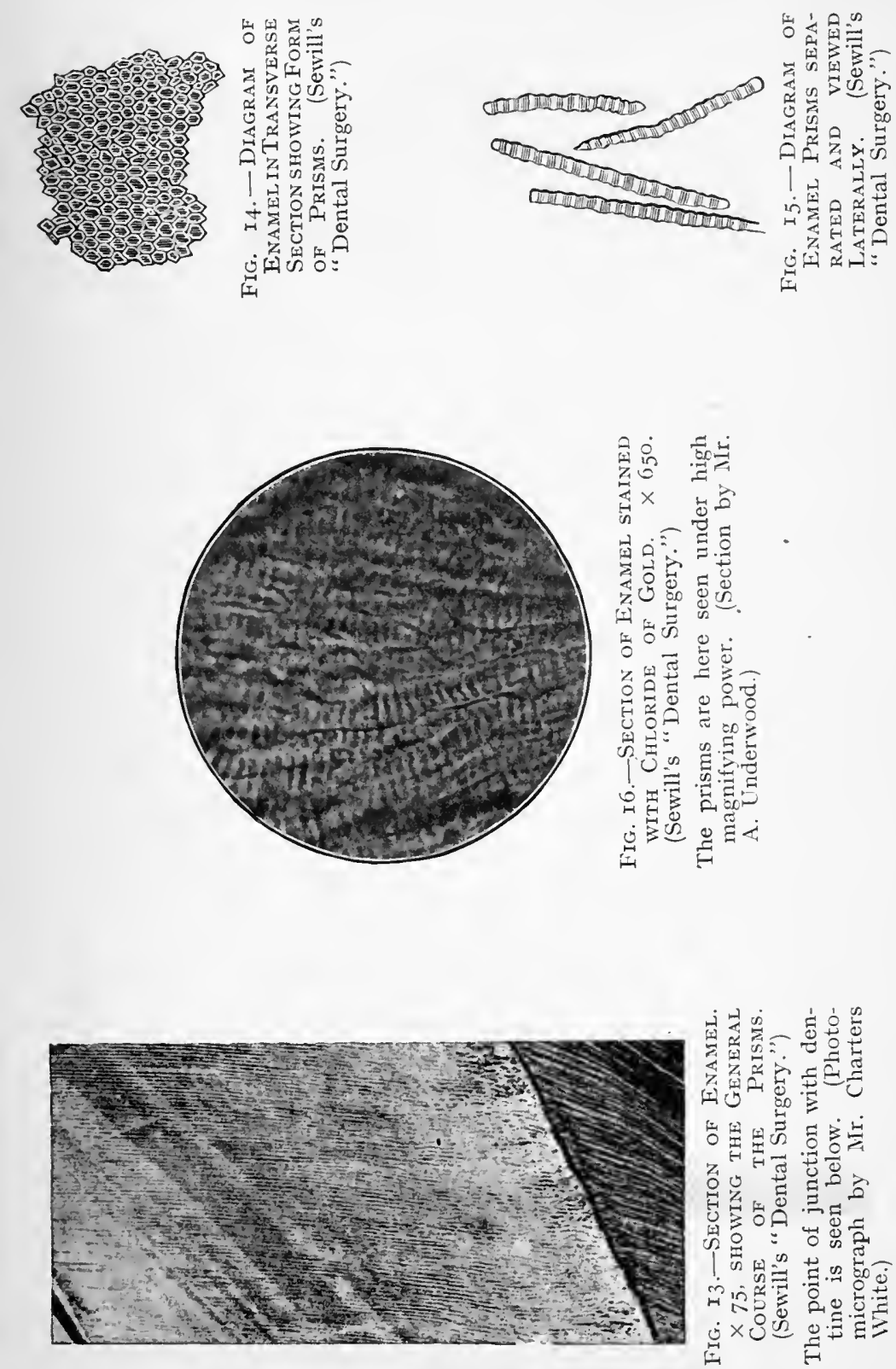


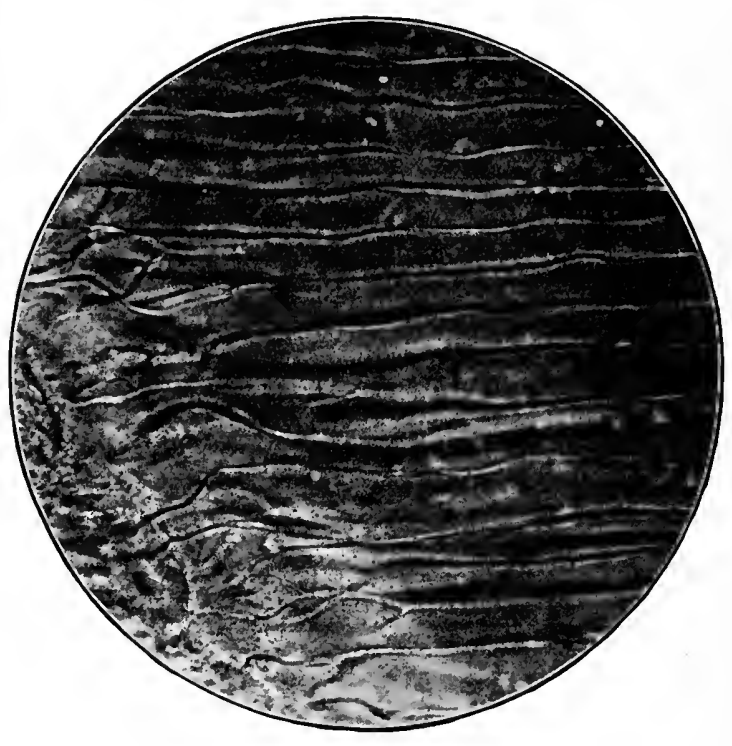

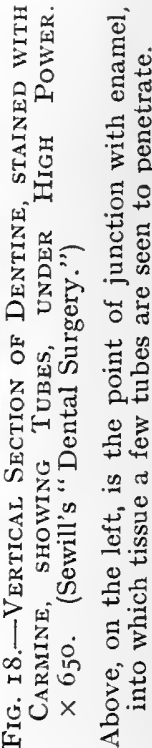
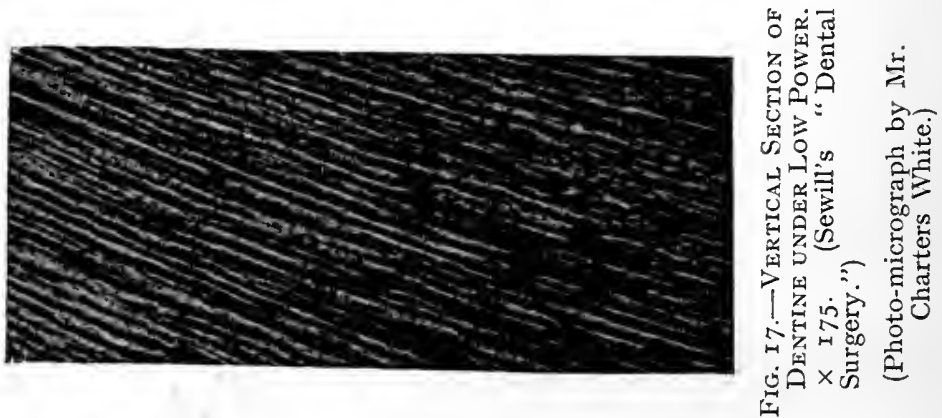
the whole tooth and roots. They run or radiate from the pulp towards the enamel of the crown and the cementum of the roots. They do not run as straight as the enamel rods, but are more or less curved in that direction. Also they are not capable of being separated like the enamel rods. This is due to their chemical composition being different. Their chemical ccmposition is similar, but the calcic salts amount approximately to 70 per cent., while the animal constituents are about 30 per cent. This will enable us to understand more clearly, later on, the stages of destruction caused by bacteria. The dentinal tubes contain nerve fibrils, which emanate from the dental pulp. These are not true nerves, but are capable of transmitting nerve sensations

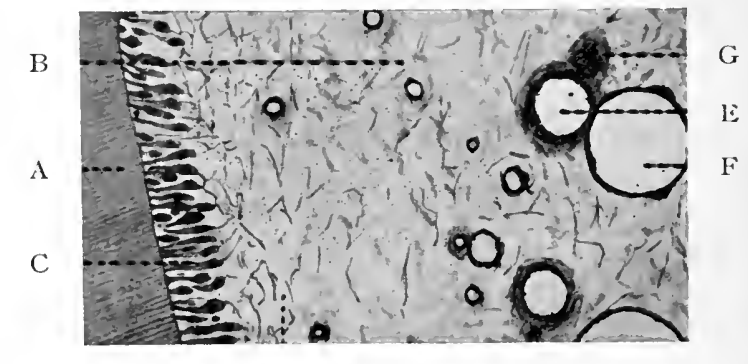

$\mathrm{D}$

Fig. 22.-The Structure of the Dental Pulp from Adult CAnine. $\times 200$. (Sewill's "Dental Surgery.")

A, Dentine; B, pulp tissue; C, odontoblasts; D, basal layer of Weil; $\mathrm{E}$, artery; $\mathrm{F}$, vein; $\mathrm{G}$, nerve fasciculus.

(From a section prepared by Mr. Hopewell Smith.)

from the enamel to the pulp. In excavating a cavity, the region immediately underneath the enamel is sometimes more productive of sensitiveness than when near the pulp itself. The tubes split up into numerous ends just against the enamel which presents that surface teeming with nerve fibril ends, which enables one to further account for the painful sensitiveness just indicated. The pulpal ends of the tubes have free openings into the internal surface of the pulp canal (Figs. I7 to 20).

The PUlP is a highly vascular organ. It is endowed with the vital essential parts belonging to the nervous and circulatory systems. It is composed of nerves and nerve matter, arteries, veins, fat, and a small percentage of other physiological and chemical substances, which are not of practical importance but of 
interest to the investigator (Fig. 22). The pulp, though important as a means of preserving aseptic conditions, seems to have no immediate or energetic function. There was a time in its existence when it was all function when forming the tooth. But afterwards, the tooth is as well off without it as with it - that is to say, the tooth in itself undergoes no change or deterioration. This is proved by the thousands of teeth that are devitalized and filled to the ends of the roots, and are just as good without the pulp as with it as far as usefulness and size are concerned. The objections to its removal involve conditions of asepsis, which is a matter relating to the periosteum, and also an artistic consideration of colour, the tooth often changing its shade considerably from absence of blood circulation the carbonizing of the contents of the dentinal tubuli.

The Cementum belongs to the root of the tooth and covers its external surface, and is of uneven thickness, being much thicker towards the apex than it is at the upper part. It is more of the nature of bone than the dentine, and is not formed by the dental pulp, but is deposited by the organ which forms the bony sockets, and which is termed the "dental periosteum" (Figs. 2 I and 23).

The teeth, considered as a complete set

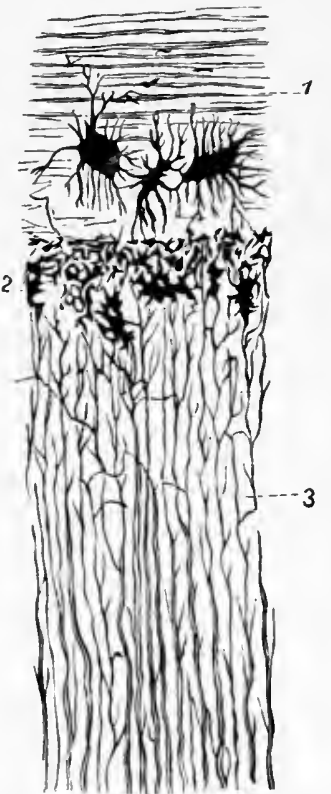

Fig. 23.-Diagrail of Transverse Section of a Canine Tooth. (Sewill's "Dental Surgery.")

I, Cementum; 2, granular layer; 3, dentine. of dental organs, have been formed, then, by three active agencies: the soft, cellular foundation of the crowns by the epithelial cords; the calcification of the crowns and the formation of the dentinal portion of the roots by the dental pulp; and finally, as if to fit them tightly into their bony cradles, the periosteum deposits bony tissue from itself to the surface of the dentinal portion of the roots. 


\section{CHAPTER IV}

\section{A DESCRIPTION OF THE IMMEDIATE NEIGHBOURING PARTS OF THE TEETH}

THE neighbouring parts of the teeth comprise:

I. The periosteum of the alveoli.

2. The basal parts of the maxillæ or jaws.

3. The antrum.

4. The gums.

5. The bloodvessels of the mouth.

6 . The nerves of the mouth.

7. The salivary glands.

8. The tongue.

9. The muscles and cheeks.

\section{The Responsibility of the Operator.}

The teeth are the immediate concern of the dental operator, but he would be frequently embarrassed if he were not thoroughly conversant with the parts surrounding these organs. To illustrate this point, if a man were working inside a building with delicate walls, and the nature of his work were dangerous to his neighbours' property, he cught to be in a position to know what was outside his immediate area, so as to control his operations. There might be a river outside or a heap of inflammatory material which he might set going. So in dental operative work there is a vast amount of tissue of an inflammatory nature which the operator might set going, and a mighty stream of blood which would be of no service in quelling the mischief. Water and fire feed each other under some conditions, but under most conditions the water quenches the fire. In surgical work inflammatory troubles are often aggravated by complications with the blood stream. But under scientific and intelligent treatment the blood contains and brings the materials for healing the trouble. Thus the dental operator must not only be able to work upon the teeth, but he must so control his operations as to protect his immediate fence or limits of his field of working. 
The limits of his work are confined to the highly important organ which lines the surface of the alveolar sockets. He should be more conscious of the presence of it than he is of the teeth. The organ to which we refer is called

\section{THE PERIOSTEUM.}

The periosteum is of vastly greater importance from a danger point of view to the operator than the teeth themselves. He can never get out of coming into contact with it, or shirk any simple or difficult operation in his daily routine without having to reckon with it. It is the constant friend of the dentist, and he has to pay very dearly sometimes for neglecting it. It is too delicate an organ to be called an enemy, and our outline of its nature and functions will show that its work is far greater than that of any other organ in relation to its size.

The derivation of the Greek prefix peri signifies around and corresponds to the Latin prefix circum. The Greek word osteon means a bone. Os is the Latin word for bone.

The periosteum covers all bone, but the lining of the dental alveoli is technically known as the pericementum or peridental membrane, which is a modified form of the periosteum. We must bear in mind that the whole structure of the teeth and alveolar portion of the jaws is a superstructure, and is more or less a temporary one. When all the teeth have disappeared, either by extraction, exfoliation by pyorrhœa, or by pericementitis, the dental portion becomes entirely absorbed right down to its original level and the basal part of the jaws is left. The pericementum not only lines the sockets but it coalesces with the gums at the necks of the teeth, so that any irritation at the necks of the teeth leads to inflammation, and readily accounts for the shrinking or absorption of the gums and bone which we commonly observe. Its origin is in the membrane that surrounds the dental follicle, which contains the enamel organ and dentinal papillæ. It is a fibrous membrane, and its fibres run from the alveolar walls to the cementum of the roots. The nerves and bloodvessels that supply it enter the membrane from the walls of the alveoli, also at the apical space and through the gingival border of the gum just below the margin of the alveolus. Nerves enter it at the same parts, so that when the operation of extraction takes place pain can easily be accounted for by the tearing 
away of the cubic surface of such a vascular and nervous tissue. The pericementum or periosteum is a very thin fibrous and vascular membrane, and performs several important functions by means of small bodies which it contains, and which characterize its existence. These are:

Osteoblasts.

Cementoblasts.

Fibroblasts.
Osteoclasts.

Lymphatic Glands.

\section{The Working Cells of the Periosteum.}

The osteoblasts are small and numerous bodies which deposit the bone around the teeth.

The cementoblasts deposit cementum around the roots of the teeth, which appears to be a composition of bony tissue and ligament.

The fibroblasts are bodies which are active when damage is done to the bone and form fibre to build up the fibrous and granular tissues of the bone prior to ossification or calcic bone deposition.

The osteoclasts are very important bodies and carry out the absorption or removal of the calcic portions of the bone. During the period of dentition and eruption, the teeth moving in such a wholesale manner within the jaws would induce a terrible condition of things if the fibro and osteoblasts with the counter action of the osteoclasts did not modify and repair the damage done. Also the same assertion applies to the operator in the operation of extraction of the teeth. The periosteum is always the friend of the operator, and puts right many serious conditions for which the operator is responsible. And when serious cases result after the extraction of badly abscessed and septic teeth it is only because the operator does not sufficiently realize the importance of the periosteum; and although this delicate organ is so laborious and enelgetic in its formative and repairing work, yet, if the septic condition of the socket is too great, it is too much for it, and leads to its active suppuration and death in its magnificent endeavours to carry out its functions.

The bloodvessels contained in it are numerous. Arteries enter the root canal at the apical end to supply the pulp, and others from four to eight in number pass along the sides of the root and divide into many branches. 
The nerves are supplied in the same manner as the arteries, and thus the peridental membrane acts as the membrane of touch to the teeth, and prevents undue pressure being exerted upon them in mastication, especially when some people abuse these organs by cracking hard nuts, breaking sticks of wood, biting thread and string, and even lifting tremendous weights by holding on with their teeth.

The lymphatics are glands whose function it is to carry off waste products and to act as absorbents.

\section{The Wonderful Qualities of Bone.}

The bones of the maxillæ, or upper and lower jaws, are designated as the superior maxillary or upper jaw, and the inferior maxillary, or the mandible, or lower jaw. Before describing these we will briefly outline the nature of the hard tissue which we call " bone." The study of bone to some of our readers might appear a very dry one, but to the dentist it cannot be so if his object is to know the highly anatomical structure on which he has to work daily, the ignorance of which leaves him sometimes mystified as to what might occur, and also as to what frequently does happen in the way of unsatisfactory sequelæ in his daily experiences. Many students are apt to decry the study of the anatomy of the lower animals, but its object is to know and discover more of what is contained in similar parts in our own bodies.

\section{Comparative Anatomy is Useful to Simplify Apparently Complicated Parts.}

If George Stephenson were able to examine the complicated locomotive engines of to-day, he would be mystified probably at the apparently complicated constructional work in them, but he would be the right man to understand the nature of those complicated parts, because he thoroughly understood the simpler principles which he himself first demonstrated to the engineering world in the locomotives which he invented and constructed. The human bones and teeth all manifest principles which are embodied in the same parts of a wide range of different classes of lower animals. So that while a demonstration may be sometimes given before a dental meeting upon such a subject as " The Teeth of Palæontological Fishes," which sounds somewhat extreme from 
a practical point of view, yet it is undoubtedly by a comparison of the various types of teeth that we get an adequate conception of the different functions of the jaws and shapes of teeth evidenced in our own.

It has been said that "whoever would become a good anatomist and a skilful surgeon must make himself a master of human osteology. It must not only be his first, but his principal and constant study. He cannot do the simplest operation in the region of bone without a competent knowledge of the bones he is operating upon."

The use of the bones is to form a framework or foundation on which the soft tissues of the body rest and carry out their working functions, and to form cavities for the lodgment and protection of delicate vessels and organs. For example, the skull protects the brain. The jaws act as supports for the masticatory apparatus with its powerful muscles, the tongue, and the firm implantation of the teeth; the orbit for the protection of the eye and the draining of the necessary bathing of that organ with the lachrymal fluid. Every part of the skeleton is built for a specific foundation purpose. If this point is clear it will help us to form a conception of the nature, shape, functions, and delicacy of the maxillæ.

As to the relative proportions of the chemical composition of bone, the best chemists agree that the calcic and earthy matter forms two-thirds, and the animal matter one-third. But it is generally accepted that while these proportions may represent the average composition of bone, yet it varies considerably at different periods of life between infancy and old age. The animal matter preponderates in early life, and the earthy matter in the decline. The strength of bone as contrasted with other substances is remarkable. It is twice as strong as oak. A piece of bone an inch square will bear $5,000 \mathrm{lb}$. weight.

Another remarkable characteristic of bone is that it gives the greatest strength with the least expenditure of material. This should be the cardinal principle on which all dentures should be made, and the clavicle or shoulder-blade is a model for dentists to study. In consequence of the animal matter that is contained in the bones they possess a wonderful degree of elasticity, and will give under great strain without breaking. We can realize this from our daily experiences with extraction of difficult teeth. If a skull be thrown on the ground it will rebound. The shoulder- 
blade is remarkably elastic, a property which enables it to break the shock of a fall upon the hand. If this bone be taken and placed at right angles against a hard substance, and the other end struck with a smart blow of a hammer, it will rebound to a distance of nearly two feet. Arab children make excellent bows with the ribs of camels. The "merrythought" of the bird affords a good instance of elasticity. It acts as a spring, and restores the base of the wings to their proper position after the action of the muscles of flight. The parts of bone are numerous, but none the less important, as every bone is made up of them. These include eminences, depressions, processes, tuberosities, spines, foramina, notches, canals, sinuses, fossæ, condyles, etc. The general characteristics of the structure of bone are an outer plate and an inner cancellated or recticular process. In some bones the centre is filled with a soft, pulpy, fat substance called marrow. These layers, though differing in density, are intersected with canals, which carry main nerves, arteries, and veins with their branches. They are also further honeycombed with holes which carry the multitudinous fine endings of these vessels called capillaries.

\section{Further Accentuation of the Periosteum with Reference to Damaged Bone.}

Everywhere, except at the insertion of strong tendons and where covered with strong cartilage, the bones are invested with a tough fibrous tissue called the periosteum. Its chief use is to provide a bed in which the bloodvessels may divide and subdivide, and so reduce themselves to a size small enough to penetrate the pores on the surface of the bones. In early life it ministers to the formation and growth of the bone in thickness, and ever afterwards to its nutrition and repair. If, therefore, the periosteum be torn from the surface of a bone, there is risk that a layer of the subjacent bone will lose its vitality, and the necrosed portion be thrown off. Though bone in health has but little sensibility, yet in disease or when damaged it becomes exceedingly sensitive. There is even such a thing as neuralgia of the bone.

If a portion of periosteum be detached or torn away by injury or disease from the surface of a bone, as it sometimes is in extraction of teeth, especially abscessed ones, a part of the thickness of 
the adjoining bone will be in great danger of dying. But it will not necessarily die, because its bloodvessels may still be filled from within, owing to the free communication between the bloodvessels of the periosteum and those of the interior of the bone. Although the periosteum is the principal organ for the repair of the bone, yet it is not the only means by which repairing of it can be carried out. Wherever Nature requires bone to exist, she can form it out of surrounding tissue in combination with the blood. In the repairing process of the sockets after extraction of teeth, or the accidental breaking away of pieces of bone, the blood clot forms a granulation of a fibrous and cartilaginous nature, and then deposits within this the required earthy salts.

\section{The Bones of the Skull.}

The bones of the skull are divided into cranial and facial bones. There are eight cranial:
I Occipital.
2 Parietal.
I Sphenoid.
2 Temporal.
I Frontal.
I Ethmoid.

Also fourteen facial:

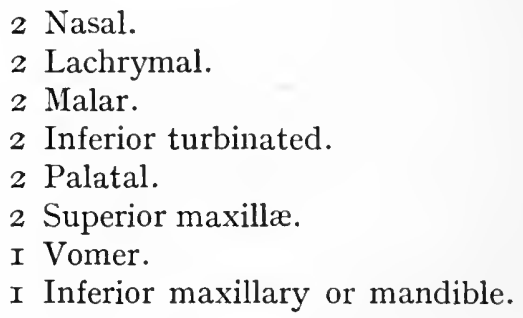

The bones of importance in our work are:

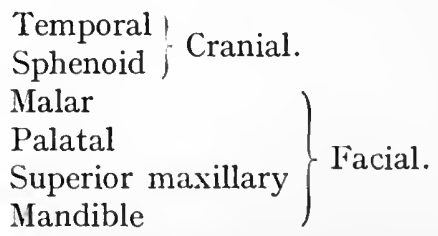

For convenience in explaining them we will alter their order and also treat them singly.

The malar bone (see Fig. 29) forms the prominence of the cheek at the corner of the face. Its top edge forms part of the 
rim of the orbit, occupying about five-twelfths of the total circumference of the orbit, involving the lower and also the outer rim at the side. The bottom part of the bone forms a rim that stands right away from the superior maxilla. Its back edge commences about two-thirds up the side of the orbit, where it joins the frontal bone and runs down towards the bottom edge; it then takes a turn at a right angle to the back edge at this point, and runs parallel to the bottom edge with it, forming a suture with the zygomatic process of the temporal bone. Its surface contains the points of origin of the minor and major zygomatic muscles and part of the masseter muscle (see chapter on Muscles).

The temporal bone is involved with the mandible in a very important way. The important parts of this bone to us are:

I. The glenoid cavity.

2. The zygoma.

3. The squamous portion.

4. The mastoid process.

5. The styloid process.

6 . The petrous portion.

The position of the temporal bone occupies the temple. It is a very complicated but interesting structure (Fig. 24).

The glenoid cavity is the socket in which is placed the condyle of the mandible (see Temporo-Mandibular Joint in the chapter on Muscles). The temporo-mandibular joint is important in considering dislocation of the lower jaw. The zygoma is a rail-shaped part that runs out and away from the squamous portion and joins the back edge of the malar bone. These two parts of the two bones form the zygomatic fossæ which contain grooves to accommodate powerful muscles of mastication, and which are also involved in dislocation of the mandible. The under edge of the zygoma towards the back part is shaped into a deep circular-shaped ridge, which forms the front rim of the glenoid cavity and is called the eminentia articularis. The zygoma carries the position of the origin of part of the masseter muscle.

The squamous portion is a large, more or less round, flattened surface like a large scale. It is situated above the zygoma, the glenoid cavity, and the petrous portion. Its wide surface takes the origin of the temporal muscle. 
The mastoid portion is situated just behind the orifice of the passage of the ear. It is nodular in shape and forms the corner at the back and base of the cranium. It is sometimes the seat of serious septic trouble. The interior is of a coarse, spongy structure, and is ultimately connected with the interior of the ear.

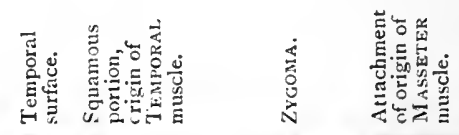

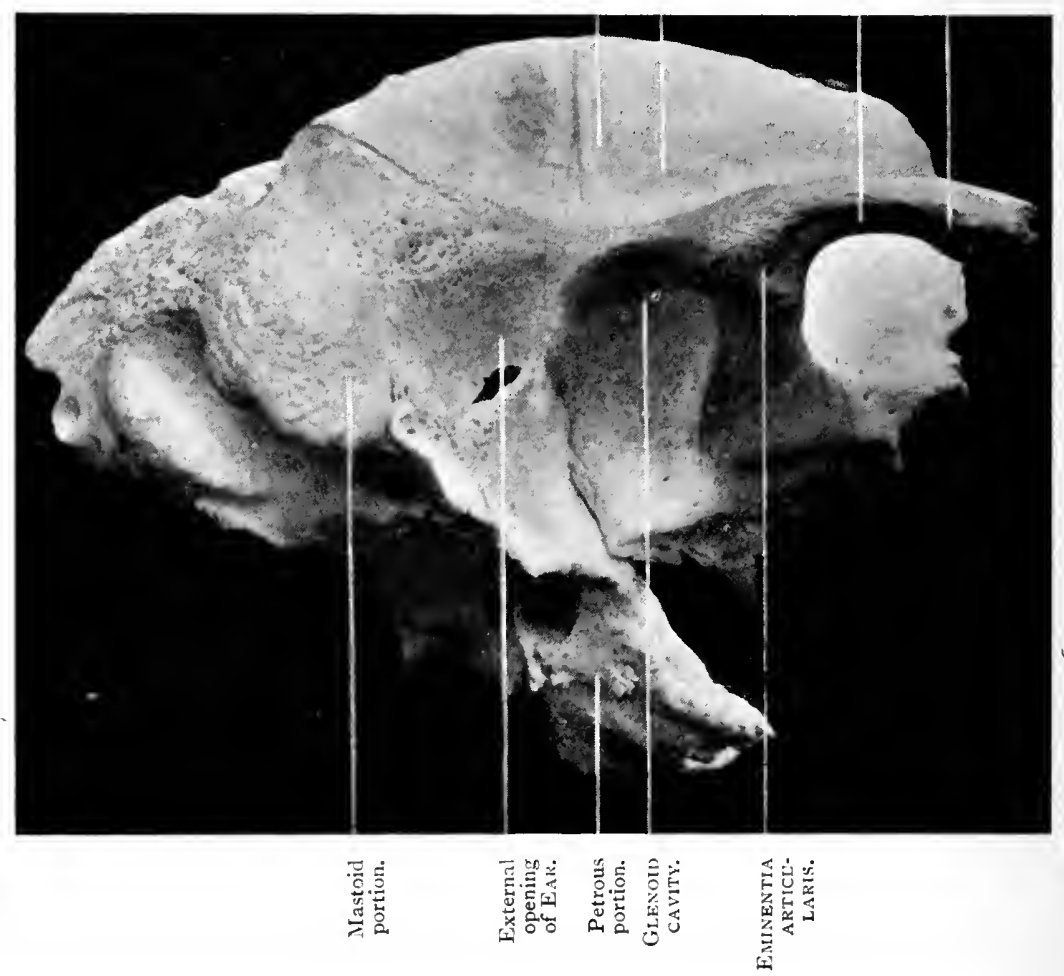

Fig. 24-The Temporal Bone.

The styloid process, so called from its resemblance to a pen, is a peg-like bone which is inserted almost immediately behind and inside the neck of the mandible and immediately beneath the orifice of the ear. It looks like a long, thin root of a tooth. It varies in length and beccmes hard as age advances in different people. On its extreme end is the origin for the stylo-mandibular 
ligament. The petrous portion derives its name from the hardness of its bone. It contains the ear and runs along towards the centre of the base of the cranium, both bones meeting within an inch of each other. On the extreme top end of the petrous portion is a depression in which the gasserian ganglion lies. The temporal bone joins the parietal, sphenoid, cccipital, and malar bones.

The sphenoid bone is so called because it is wedged in at the base of the skull and forms a strong supporting beam to the bones of the cranium and face. It not only enters into the formation of the inner base of the cranium, the orbits, the temples, and the nasal passages, but it is also connected with all the cranial bones and many of the facial bones. Its general shape bears a remarkable resemblance to a bat with extended wings, with a body or central part, two greater wings, two lesser wings, and the pterygoid processes which make the legs of the bat (Fig. 25). This bone has several foramina or apertures which transmit bloodvessels and nerves from the brain; but there are three important ones for us to notice, because they transmit the three great branches of the trigeminal or fifth pair of nerves. They are:

r. The Sphenoidal Fissure.

2. Foramen Rotundum.

3. Foramen Ovale.

These apertures are situated in the top part of the sphenoid bone, which forms part of the floor of the cranium.

The pterygoid process or plates are situated on the lower outside part of the legs and stand out at the corners. The process consists of two plates, which take the origin of the internal and external pterygoid muscles. The pterygoid processes are closely joined to the palate bones, and also act as buttresses to support the superior maxillary bones and form a frame for the posterior openings of the nose.

\section{Description of the Lower Jaw for Practical Purposes.}

The inferior maxilla, also called the mandible or lower jaw, is a separate bone, complete in itself, and is not attached by a suture or a join to any neighbouring bone. Its extreme ends shape off to a very small round shoulder or condyle, called the neck or condyle, which acts as a joint or hinge for its working, 
and fits or rather oscillates in the glenoid cavity, which is situated immediately in front of the centre of the ear, where it can be felt by touching it during mastication. About an inch and three-

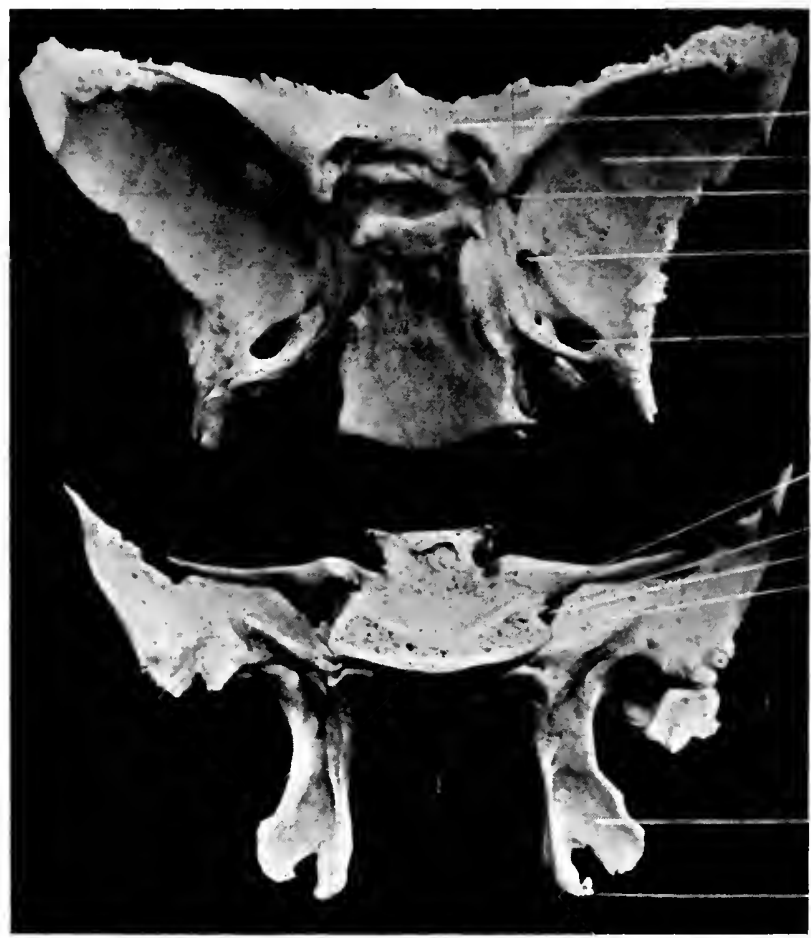

Fig. 25.-The Sphenoid Bone.
Lesser wing.

Greater wing.

SPHENOIDAL fissure.

ForameN

ROTUNDUM.

FORAMEN OVALE.

Iesser wing.

Greater wing.

SPHENOIDAL fissure.

Cerebral surface.

\section{External} pterygoid plate.

Internal pterygoid plate.

Hamular process, origin of superior constrictor muscle of pharynx.

Exhibited in two positions. The upper one shows the whole of its cerebral or brain surface; the lower one is viewed vertically, showing the top outline of cerebral surface, the sides which carry the pterygoid muscles, and the central lower part which constitutes the roof and sides of the nasal cavity.

quarters in front of the neck is a similar spike-shaped end called the "coronoid process," but it does not act as a joint (see Figs. 26 and 27 ).

The slipping forward of the neck or condyle causes the accident, which we occasionally come across, termed dislocation of the jaw. Dislocation may be either unilateral or bilateral. If one side slips out it is unilateral, and is usually soon put back into its socket. But if both sides are out it is called bilateral, and is 
between the malar rail, and at the same moment this movement tilts the whole jaw downwards in front, and the patient presents a distressing appearance. By putting a napkin on the back teeth in the lower, then standing behind and over the patient,

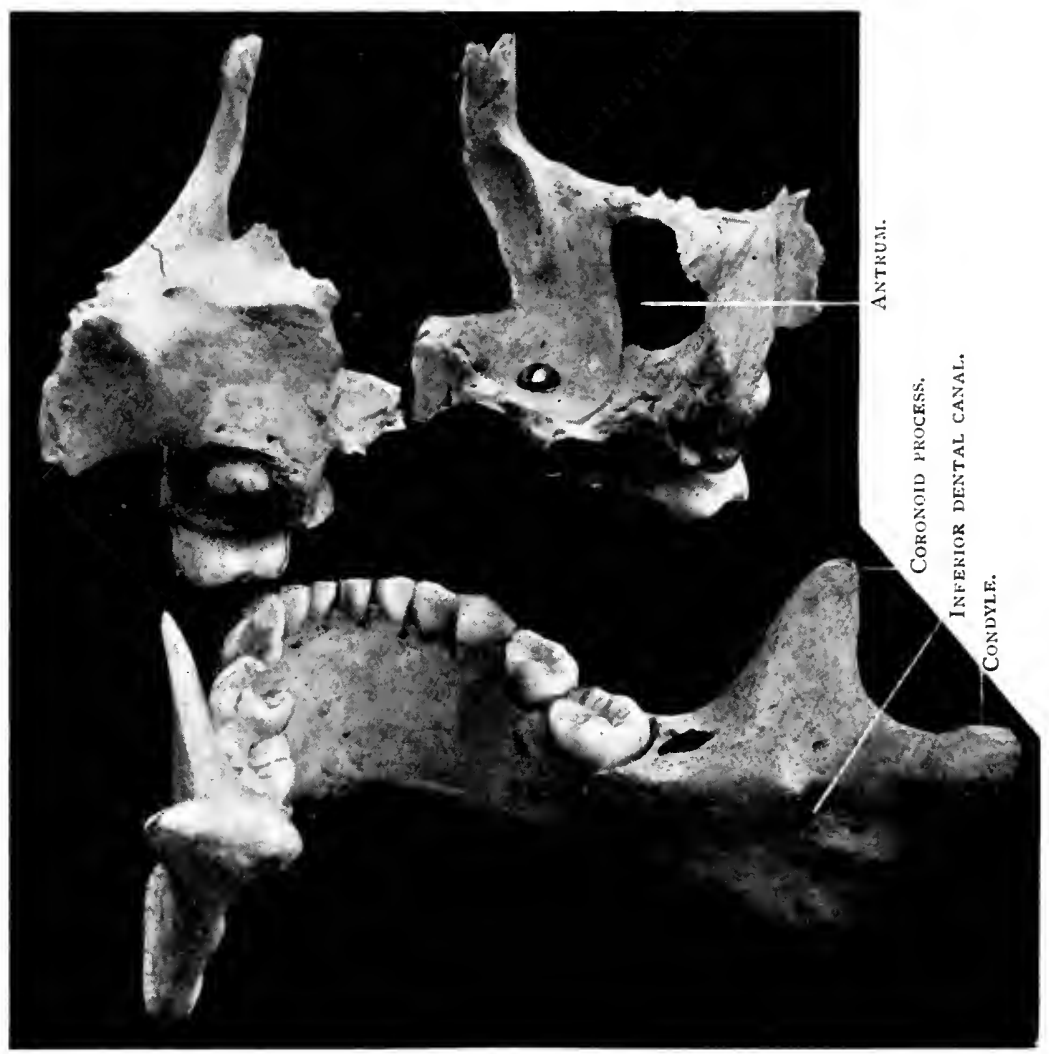

Fig. 27.-The Superior aNd INferior Maxillary Bones of a Child AGED $5 \frac{1}{2}$ YEARS.

Viewed from behind. (Photograph taken from the same specimen as in Fig. 26.)

and forcibly gripping the sides of the jaw with thumbs on the napkin and forcing it downwards and backwards so as to overcome the powerful contraction of the muscles, at that moment it will slip into its place in the sockets of the glenoid cavity (see Figs. 32 and 34 ). 
The most important feature of the mandible, however, which we should not miss, is the inferior dental canal. This is a most important part, and is always likely to be involved in the extraction of lower teeth as well as in root treatment. It carries the very large nerve called the inferior dental nerve, and the large bloodvessels called the inferior dental artery and vein. They enter the canal through a large foramen or opening at a point on the inner or tongue side of the jaw about one inch. behind the lower wisdom teeth and a little above the level of them. It then dips down into the basal part of the jaw, just missing the roots of the wisdom teeth and runs about a quarter of an inch below the roots of the molar and bicuspid teeth until it reaches the second bicuspid. At this spot it branches into two parts, the larger branch coming out at a hole called the mental foramen, and enters into the corners of the lower lips. This branch sometimes absorbs cocaine or other alkaloid solutions, especially if containing much adrenalin, and will cause local anæsthesia in this part, and the patient complains of something the matter with the lip. The other branch, called the incisive branch, continues from the second bicuspid into a much smaller canal in the jaw, in a line with the back part of the canal, and ends under the central teeth. The nerves and vessels of one side do not have any connection with those of the other side. The lower jaw is really a flattened tube. The outside part is called the cortical plate, and the interior the alveolar process and the spongy process. There are fourteen pairs of muscles attached to the mandible.

\section{Description of the Upper Jaw for Practical Purposes.}

The superior maxilla or upper jaw is of quite a different form, and, beyond the fact of its containing the upper teeth, it has no similarity to the mandible. In the first place it is much smaller in width and has not anything like the mass or depth of the lower. Of course this is accounted for by the fact that it is strengthened by being connected with several neighbouring cranial bones, and is also strengthened by the palatal bone which is an extension of the upper jaw, each forming a floor to the nasal cavity and a vault to the mouth. These bones made up of one on each side joining in the centre of the face and called the superior maxillæ, are the most important bones of the face 
excepting the mandible, and form, by their union in the centre of the mouth, the whole of the upper jaw. Each side assists in the formation of three distinct cavities, viz.:

The roof of the mouth,

The floor and outer wall of the nose, and

The floor of the orbit or eye socket.

Each bone consists of a body and four processes, viz.: Zygomatic, Alveolar, Frontal, Palatal (Figs. 28 and 29).

\section{The Antrum constitutes the Body of the Upper Jaw.}

The body of the jaw is somewhat pyramidal in shape, and contains a large cavity called the antrum, or the maxillary sinus.

Also five surfaces as follows:

I. An anterior or facial surface from the-floor of the orbit forming the front of the face on each side to the alveolar border, and slightly concave, excepting at the eminences above the roots of the teeth.

2. A posterior or zygomatic surface, downward, backward, and outward, forming the sides of the lower part of the face from just under the cheek prominence to midway between the nose and ear. It ends just against the coronoid process of the lower jaw, and just inside it.

3. The superior or orbital surface is smooth and triangular, and forms the greater part of the floor of the orbit.

4. The palatal surface which extends from the alveolar border on one side right across to the border of the other side.

5. The internal surface, which extends from the floor of the nose and runs up each side, leading to a small opening just below the floor of the orbit. This opening is not large, and is the only opening into a large roomy cavity or chamber called the antrum. This cavity extends from the canine, or first bicuspid, right back to beyond the wisdom teeth, a distance of about one and a half inches to one and three-quarter inches. It also rises from the floor immediately above the roots of the bicuspids right back to the wisdom teeth upwards to immediately underneath the orbits. In children these measurements are much less. The floor of the orbit is so thin that any swollen condition of the eye would depress it on to the antral space. On the other hand, if the antrum became full of pus, as it frequently does in empyema, which is an inflammatory condition of the antrum, and also in 
many nasal affections, the floor of the orbit would be pushed up. The nasal wall of the antrum is also exceedingly thin. The cavity itself would hold a quantity of pus or liquid if it were shut in.

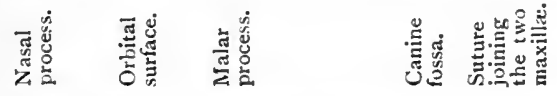
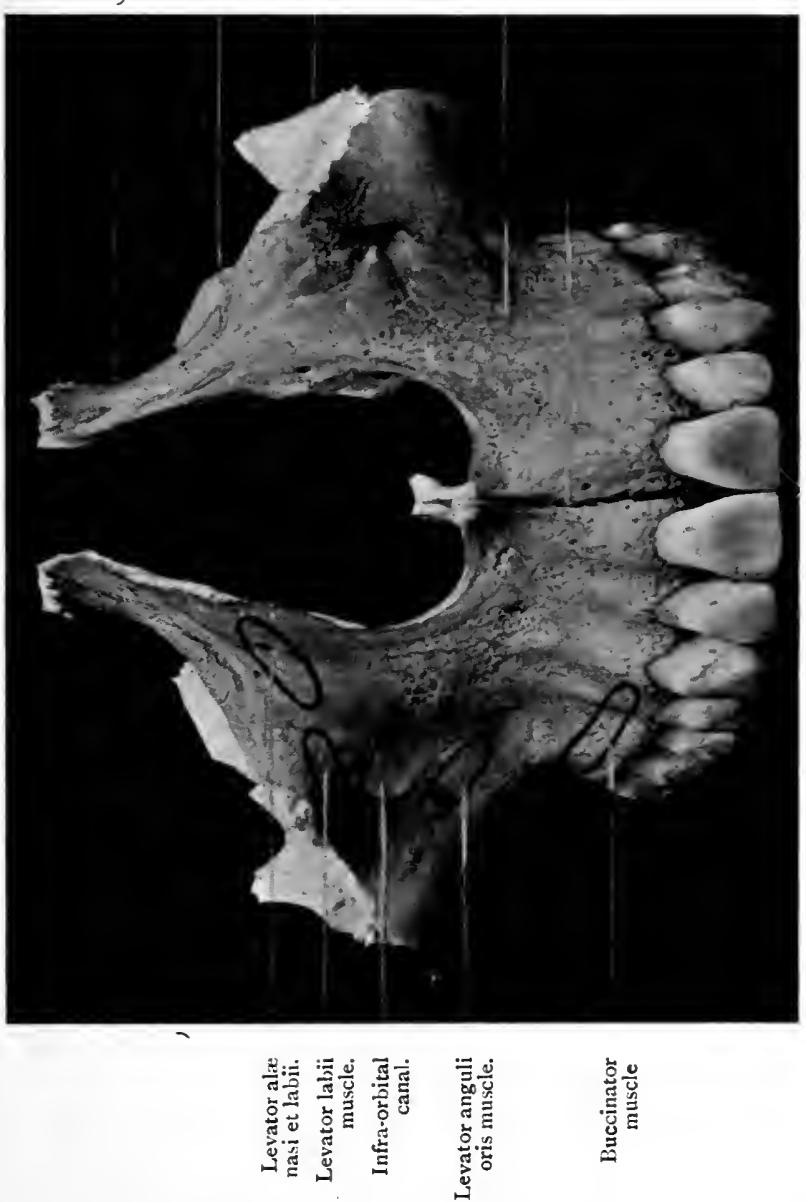

苍蒈

which is quite possible if the only opening we have described is blocked up by a swollen condition of the nasal mucous membrane at that point, to the extent of from a teaspoonful in a child to two drachms in an adult (see Fig. 34). 
The extent of the antrum on each side leaves very little actual solid bone in ally part of the upper jaw (sce Figs. 32 and 34). It is fairly thick in front from canine to canine, but that is all. At
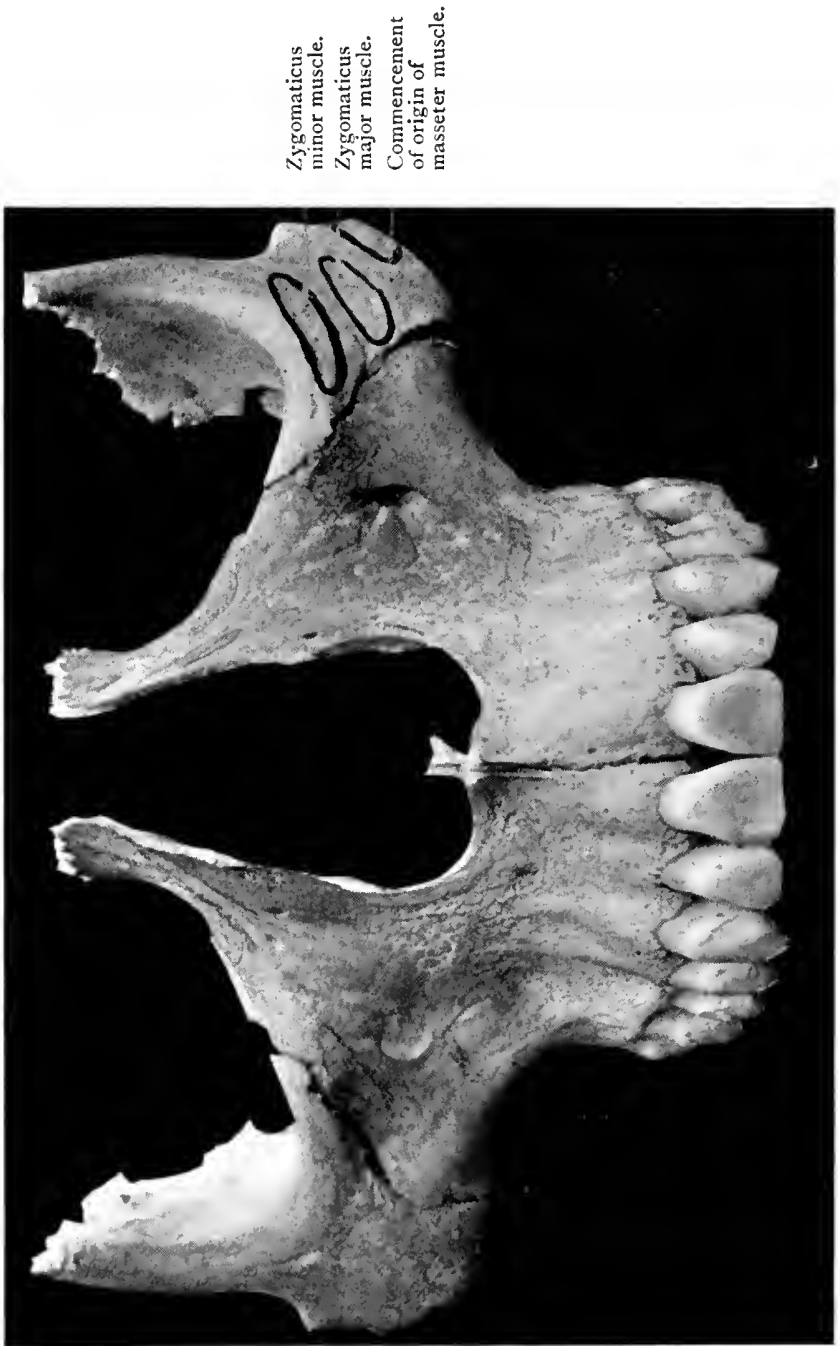

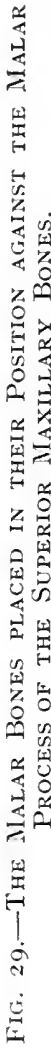

every other part it consists of a plate only, of more or less even thickness, and indeed, at a point in the region of the second bicuspids in the palate right at the top at each side, a point 
could easily be pushed into the chamber. This spot is of no utility for draining purposes, as it is right above the floor, and there is also great danger of striking the nerves and bloodvessels which run from the posterior palatine canals towards the anterior palatine canal. The importance of the antrum to the dentist is in extraction operations, in which an abscessed root might be pushed upwards in its alveolar socket and disappear, where it would be floating about with little chance of its being rescued without an operation of some kind, which might be simple or complex as the subsequent circumstances turned out to be. Or, again, in root treatment, probes, nerve canal drills, powerful antiseptics, etc., could easily produce inflammatory trouble in the antrum owing to its immediate vicinity to the roots of the teeth. Or, again, some judgment is necessary on the part of the operator as to whether he should interfere by operating in its vicinity, as there might already be a blocking of the small and only opening leading from the antrum to the nose, caused by a cold or empyema. His work might be blamed for causing the condition for which he would not in any way be responsible. To summarize the parts of the superior maxillæ or upper jaw, it stands thus-each side consists of:

3 CAVITIES.

Roof of Mouth.

Floor and outer wall

of nose.

Floor of the orbit.
4 PROCESSES.

Zygomatic or side.

Frontal.

Alveolar.

Palatal.

\section{SURFACES.}

Anterior or facial. Posterior or side of face.

Superior or orbital.

Palatal.

Internal or nasal.

The other important parts of the maxilla, as far as operating is concerned, are the posterior and anterior palatine canals (see Fig. 32).

The posterior palatine canal is situated at the foramen on the surface of the palate at a spot approximating to the apex of the palatine root of the wisdom tooth. It is formed by the union of the palatal bone with the maxilla. The foramen beginning at the palate is in the palatal bone and penetrates it for about fiveeighths of an inch, when the canal continues behind the antral part of the maxilla by a groove contained in it and a groove in the palatal bone. Near the top of the external wall of the antrum 
the canal opens out into the spheno-maxillary fissure which is formed by a large space between the sphenoid bone and the maxilla.

The palatal bones are two slender bones, each one shaped like a rough $L \perp$, the two bones being placed in this position. They are wedged between the sphenoid and maxillary bones, the stem of the $L$ forming the rim of the outer nasal well. The base forms the floor of the nose at the back part, while the underneath surface forms the back part of the palate and the attachment for the soft palate and the palatal muscles.

On the posterior surface of the maxilla, on the outer wall approximating to the face and about two-thirds of the height of the maxilla, foramina may be seen which transmit the posterior dental nerves which run down to supply the roots of the molar teeth (see chapter on Nerves). The anterior palatine canal is situated immediately behind the upper central teeth. The canal is formed by a groove situated in the right and left maxilla. The palatal foramen is very large, but becomes somewhat smaller as it runs upwards, and ends with two smaller but short canals called the foramina of Stensen, forming the letter $Y$ with a long stem. There are several small foramina, some situated in the front of, and also behind, the canal, but branching from the canal, which transmit the naso-palatine nerves, and are called the "foramina of Scarpa." Great care must be taken to avoid plunging a hypodermic needle into the anterior palatine canal, because it is usually situated right against the necks of the central teeth. The same precaution must be used towards the posterior palatine canals, although they are not so contiguous to the wisdom teeth.

The superior maxillary bones are attached to about nine bones: two of the cranium-viz., the frontal and ethmoid, and seven of the face-viz., the nasal, malar, lachrymal, inferior turbinated, palatal, vomer, and sometimes the sphenoid.

\section{The Nature of the Gums, with Indications where Care is Needed.}

The gums consist of the linings that cover the external surfaces of the jaws within the oral cavity. They are composed of a dense fibrous tissue closely connected with the peridental membrane of the alveolar processes and surrounding the necks 
of the teeth. They are covered by smooth and vascular mucous membrane, which has a limited sensibility. Around the necks of the teeth this membrane presents numerous fine papillæ, and is reflected into the alveoli, where it is continuous with the periosteal membrane lining the sockets. It consists of the mucous membrane, which is the lining or the skin of the internal passages of the body. Its surface is covered with its secretion called mucus, which is of a gummy, tenacious consistency, and which serves to protect it from foreign substances with which it is brought into contact. The mucous membrane consists of four different layers:

\section{The Epithelium. \\ Basement Membrane. \\ The Corium. \\ Submucous Tissue.}

The epithelium is the outer layer, and consists of two membranes called the "corneous " and the "Malpighian lining."

The basement membrane is a thin structureless lining, which separates the epithelium from the corium.

The corium is the true mucous membrane.

The submucous membrane is next to the bone, and is termed the "muco-periosteum."

The gums consist of that part of the mucous membrane which surrounds the teeth, and which is thicker than the other parts. It will be seen that the mucous membrane is intimately attached to the bone, and there is danger of a suppurating character if it should be badly torn in extraction of teeth, or lifted away by too forcible hypodermic injection, too strong rubber suction discs in the palate, and undue interference with the gums when regulating children's teeth. When the gums are thus lifted or peeled away frcm the bone a thick viscid serum collects, which will sometimes spread its way along the bone. Instances of this can sometimes be seen in a case of a palatal abscess, where, instead of breaking at a fistulous opening or gumboil, it forces up the mucous membrane, and there is danger of it falling into the throat ultimately. 


\section{CHAPTER V}

\section{A DESCRIPTION OF THE IMMEDIATE NEIGHBOURING PARTS OF THE TEETH-(Continued)}

\section{The Circulatory System.}

IT is very important that the dental operator should understand where the bloodvessels of the mouth lie, but it is infinitely more incumbent that he should constantly bear in mind the nature of the fluid that passes through them; also what sequelæ might probably arise from infection when a bloodvessel is opened either by operation or by accident. When injections are made these bloodvessels are frequently punctured, whereas by a little observation it will be noticed that superficial vessels vary in number and position with every patient.

On the labial or outside part of the gums the superficial veins can easily be seen lying in the upper or lower half of the gums between the lighter pink zone around the necks of the teeth up towards the sulci, or that part of the lips or cheeks which folds over and is composed of loose cellular tissue, which is not only dangerous for plunging hypodermic needles into, but is useless for securing local anæsthesia with the small doses that the dental operator should use. On the lingual side of the gums there is only a very limited zone for safe hypodermic injection, which is just around the necks of the crowns of the teeth for a depth of about a quarter of an inch.

When describing the upper jaws or maxillæ we noted the position of the posterior canals which have a large opening in the palatal surface of the bone just in the region of the apices of the wisdom teeth. They are situated on the level of the floor of the palatal bone, at a point where it curves down towards the wisdom teeth. In some jaws this spot is three-quarters of an inch from the gum margin, in others it is about half an inch. Its bore or diameter is about one-eighth of an inch and is somewhat larger at its immediate opening at the surface of the palate. Again, there is the anterior palatine canal, which is situated immediately behind the upper central teeth. This is much 
larger than the posterior canals in bore or diameter, and is in immediate danger of being punctured or damaged. These canals allow the passage of an artery, vein, and nerves. From the posterior canals emerge bloodvessels, which run forward in a groove on the surface of the hard palate.

The position of the descending palatine artery should be borne in mind when using dangerous instruments such as a sharp elevator, as it is in danger of being wounded and may give rise to formidable hæmorrhage, while in rare cases it has been found necessary to plug the canal itself in order to arrest the bleeding.

We are constantly aware of the readiness which the tissues have of bleeding. The amount of blood that appears may be much or little; if much, it probably comes from bloodvessels which could be seen easily with the naked eye, and, on the other hand, if there is little blood it probably comes from bloodvessels of so small a size that they are termed arterioles, which become finer still and are called capillaries. In the extraction of teeth blood flows in many degiees of force and quantity. When the bleeding has subsided, the clot becomes the centre from which repair of the tissues commences, and which is ultimately completed through the agency of the blood, which is constantly bringing materials for the wcrk, and which also carries away the waste products from the process. The same thing is going on in every part of the mouth in health, and also in every part of the body. We shall therefore defer the location of the bloodvessels of the mouth to another chapter, and briefly describe the apparatus of the circulatory system and the fluid that passes through it, which is called blood. The following description has been prepared for our readers from Gray's “ Anatomy," Halliburton's "Physiology," and other standard works.

\section{Some of the Foregoing Reasons should Lead us to Enquire as to the Nature of the Circulatory System.}

Bloodvessels are divided into three kinds, known as arteries, capillaries, and veins. They are entirely different in their functions, and form, with the heart, the blood vascular system. The branch of anatomy which deals with the circulation of the blood is called angiology. This comprises a description of the vascular system of the body and is divided for descriptive purposes into:

I. The blood vascular system, and

2. The lymph vascular system. 
The blood vascular system consists of the heart and bloodvessels for the circulation of the blood.

The lymph vascular system consists of lymphatic glands and vessels, through which a colourless fluid called lymph circulates. It must be noted, however, that the two systems communicate with each other, and are intimately associated developmentally.

\section{The Heart and its Function.}

The heart is the central organ of the blood vascular system, and consists of a hollow muscle; by its contraction the bleod is pumped to all parts of the body through a complicated series of tubes called arteries. The arteries undergo enormous ramification in their course throughout the body, and end in very minute vessels called arterioles, which in their turn open into a closely meshed network of microscopic vessels called capillaries. After the blood has passed through the capillaries it is collected into a series of vessels which in turn open out into larger ones; these are called veins, by which it is returned to the heart. The passage of the blood through the heart and bloodvessels constitutes what $\mathrm{s}$ termed the circulation of the blood.

The human heart is divided by a wall into right and left halves; each half is further divided into two cavities: an upper, called the auricle, and a lower, termed the ventricle. The heart, therefore, consists of four chambers, called the right auricle and the right ventricle, forming the right half; then the left auricle and the left ventricle, forming the left half.

The auricles are receiving chambers and the ventricles are distributing ones. The right half of the heart contains venous or impure blood; the left contains arterial or pure blood. From the cavity of the left ventricle the pure blood is carried into an artery of tremendous capacity called the aorta, through the large branches of which it is distributed to all parts of the body, with the exception of the lungs.

\section{The Object of the Circulation of the Blood to nourish and purge the Cell.}

In its passage through the capillaries of the body the blood gives up to the tissues the materials necessary for their growth and nourishment, and at the same time receives from the tissues the waste products resulting from their metabolism. Living 
material is in a continual state of unstable chemical equilibrium, building itself up on the one hand, and breaking down on the other. The term used for the sum total of these intra-molecular rearrangements is metabolism. The chemical substances in the protoplasm with which the multitudinous cells of the body are filled contain complex nitrogenous compounds called proteins. So far as is at present known protein material is never absent from living substance, and is never present in anything else but that which is alive or has been formed by the agency of living cells. It may therefore be stated that protein metabolism is the most essential characteristic of vitality. To understand the nature of the tissues of the various parts of the body that are nourished by the blood we must again refer to the cellular nature of them. In our description of the teeth cells formed during the earlier stages of dentition, we endeavoured to show how those parts were built from simple forms to more complex ones. The same development applies to all tissues, whether hard or softmuscle-tissue or bone-tissue-and thenceforward in the adult, when all development has subsided, those final cells of the body have to be maintained by the processes of nourishment and metabolism that are now being described.

The adult body consists of a great number of different parts, and each part has its own special work to do. Such parts of the body are called organs. Each organ does not only its own special work, but acts in harmony with other organs. This relationship between the organs enables us to group them together into what are termed systems. Thus we have the circulatory system, which consists of the group of organs-heart, arteries, veins, arterioles, and capillaries-concerned in the circulation of the blood; the respiratory system, which includes the group of organs-air passages, lungs, nerves, etc.-concerned in the act of breathing; the digestive system, which deals with the digestion of food; the excretory system, which is engaged in getting rid of waste products; the muscular system, which works the movements of the body; the skeletal system, which supports the soft parts of the body. Over and above all this is the nervous system-brain, spinal cord, and nerves-which constitutes the great master system of the body, and which presides over, controls, and regulates the functions of all the other systems.

If we proceed to make an anatomical analysis, and take any organ, we see that it consists of various textures, or, as they are 
called, elementary tissues. Just as one's garments are made up of textures, cloth, lining, buttons, etc., so each organ is composed of corresponding tissues.

The elementary tissues come under the following four headings:

I. Epithelial tissues.

2. Connective tissues.

3. Muscular tissues.

4. Nervous tissues.

Each of these is again divisible into sub-groups. If we continue our anatomical analysis still further, we shall find that the individual tissues are built up of structures which require the microscope for their accurate study. Just as the textures of a garment are made up of threads of various kinds, so also in many of the animal tissues we find threads or fibres as they are called. But more important than the threads are little masses of living material. Just as the wall of a house is made up of bricks united by cement, so the body walls are built of extremely minute living bricks, united together by different amounts of cementing material. Each one of these living units is called a cell. Some of the tissues already mentioned consist of cells with only very little cement material binding them together; this, for instance, is seen in epithelial tissues; but in other tissues, particularly the connective tissues, which are not so eminently living as the rest, the amount of cement or intercellular material is much greater, and in this it is that the fibres are developed that confer the necessary strength upon these binding tissues.

If, instead of examining the adult animal, we look at the animal in its earliest stage of development, the ovum, we find that it consists of a single little mass of living material, a single cell. As development progresses it becomes an adherent mass of cells. In the later stages of development various tissues become differentiated from each other by the cells becoming grouped in different ways, by alteration in the shape of the cells, by deposition of intercellular matter between the cells, and by chemical changes in the living matter of the cells themselves. Thus, in some situations the cells are grouped together into the various epithelial linings; in others the cells become elongated and form muscular fibres; and in others, as in the connective tissues, there is a preponderating amount of intercellular tissue which may be permeated with fibres, or be the seat of the deposition of calcium 
and other salts, as in bone and the teeth. Instances of chemical changes in the cells themselves are seen on the surface of the body, where the superficial layers of the epidermis become horny; in the mucous glands, where they become filled with mucin; and in adipose tissue, where they become charged with fat.

The term "cell" was first used by botanists. In the popular sense of the word a cell is a space surrounded by a wall, as the cell of a prison or the cell of a honeycomb. In the vegetable cell there is a wall, made of the starch-like material called cellulose. Within this wall is the living matter and a number of large spaces or vacuoles filled with a watery fluid. But the animal cell is different. As a rule, it has no obvious wall, and vacuoles are not conspicuous. It is just a little lump of living material. This living material is jelly-like in consistency, possessing the power of movement; the name of protoplasm has been bestowed upon it. Somewhere in the protoplasm of all cells, generally near the middle in animal cells, is a roundish structure of more solid consistency than the rest of the protoplasm, called the nucleus. An animal cell, therefore, may be defined as a mass of protoplasm containing a nucleus (see Chapter II., p. I6).

\section{Venous Blood and Pulmonary Circulation.}

The circulation of the blood, therefore, is to nourish the tissues of the body and carry away the waste products. In doing so it becomes changed from arterial into venous blood, which is collected by the veins, and through them returned to the right auricle of the heart. From this cavity the impure blood passes into the right ventricle and is thence conveyed through the pulmonary arteries into the lungs. In the capillaries of the lungs it again becomes arterialized, and is then carried to the left auricle by the pulmonary veins.

\section{Arterial Blood and Systemic Circulation.}

From the left auricle it passes into the left ventricle, from which the cycle once more begins. The course of the blood from the left ventricle through the body generally to the right side of the heart constitutes the greater or systemic circulation, while its passage from the right ventricle through the lungs, where the venous blood has been oxygenated or arterialized, to the left side of the heart, is termed the lesser or pulmonary circulation. 
The heart is a hollow muscular organ, situated in the thorax or chest, between the lungs, and enclosed within a bag.called the pericardium or pericardial sac. It rests on the diaphragm somewhat to the left of the median or centre line of the chest. It is conical in form, and is suspended by the great vessels in such a manner that the apex points to the left and downward. Its size is about that of the closed fist, and its average weight is about ten ounces. It is divided by a septum, or wall, into two cavities not connected with each other, in each of which is contained an auricle or blood-receiving chamber and a ventricle or blooddistributing chamber. The capacity of the auricles is about four ounces, and that of the ventricles about six ounces. This organ is controlled by two sets of nerves, which are branches from the pneumogastric or vagus nerves and the sympathetic nerves. The vagus nerves have a slowing or inhibitory effect and the sympathetic have an acceleratory or quickening effect on the heart's action. The rhythmical action of the heart is muscular in origin; that is to say, the heart muscle itself possesses the inherent property of contraction apart from any nervous stimulation. The nerves, although not concerned in originating the contractions of the heart muscle, play an important part in regulating their force and frequency in order to subserve the physiological needs of the body. The valves of the heart allow the blood to pass in one direction only, and thus prevent regurgitation. If there is any sign of regurgitation, as indicated by the stethoscope, it is because the valves do not close completely, which is a sign that there is something wrong in their working action. The movements of the heart are termed systole or contraction, and diastole or expansion. It beats at the rate of I 40 to I5O before birth, I30 at birth, during first year I25 to I35, during third year 95 to Ioo, eighth to fourteenth year 85 to 90 , adult about 75 , old age 65 . The beats of the heart may be heard by using a stethoscope; the first sound, over apex of heart, is dull, prolonged, and bocming; the second, immediately following the first, is sharp, quick, and almost clicking, and is heard over the base of the organ. Then follows a momentary rest, called the period of repose, so that the heart, while apparently constantly beating, gets a momentary rest at the end of the second beat. The heart sounds are caused, first, by the muscular action and vibration of the auriculo-ventricular valves and the chordæ tendineæ; the second is caused by the closure of the semilunar 
valves. The valves of the right ventricle are the tricuspid and the pulmonary, and in the left ventricle the bicuspid or mitral and the aortic. The left ventricle exerts more than twice as much power as the right. The aortic valve consists of three semilunar segments which surround the orifice of the aorta. They are similar in structure to those of the pulmonary valve. The chordæ tendineæ are connected to both cusps of the bicuspid or mitral valve. The influence of the nervous system upon the heart's action is not clearly known. The mechanism of rhythmical contraction is contained within the heart itself, and it is possible that nerve ganglia exist in the human heart as they do in the frog's heart, and these ganglia are connected with fibres from the pneumogastric or vagus nerves and with the sympathetic system. Experiments have shown that if the fibres from the pneumogastric or vagus nerves have been cut away the heart's action becomes very rapid from the acceleratory action of the sympathetic fibres. The foregoing description will probably prepare the reader for understanding the difference between fainting and syncope when signs of collapse occur in a patient. Syncope is when the action of the heart is impeded from loss of blood or by anæmia of the brain centres in the medulla, through the said action not being strong enough to pump it through the arteries leading to the brain. This can frequently be put right immediately by placing the patient into a postural or horizontal position so that the blood drains towards the head by gravitation and the heart is relieved of its vertical pressure.

Fainting is when the contractile movements of the heart are impeded by loss of nerve power, in which case nerve stimulants are indicated.

\section{The Construction of the Arteries.}

The bloodvessels which carry the blood in its circuit round the body and into the remote parts are called arteries and veins. Arteries are cylindrical, tubular vessels. They are elastic tubes, and the amount of elastic tissue is greatest in the large arteries. They contain a considerable amount of muscular as well as elastic tissue. The muscular layer co-operates with the elastic tissue in adapting the calibre of the vessels and the quantity of blood they contain. For the amount of blood in the vessels is never quite constant, and were elastic tissue only present the pressure exercised by the walls of the containing vessels on the contained 
blood would be sometimes very small and at other times too great. The presence of a contractile element, however, provides for a certain uniformity in the amount of pressure exercised. The contractibility of the arterial walls fulfils a useful purpose in checking hæmorrhage should a small vessel be cut or torn, as it assists in the closure of the cut end, and this, in conjunction with the coagulation of the blood, arrests the escape of blood. Arteries consist of three coats:

I. The external coat, which is the strongest layer.

2. The middle coat, which constitutes the greater part of the arterial walls.

3. The internal coat; its inner surface is lined with an exceedingly smooth layer so that the blood may flow with the smallest amount of resistance from friction.

The walls of the arteries are, like other parts of the body, supplied with bloodvessels which extend for some distance into the external and middle coat but do not reach the internal coat. Most of the arteries also are surrounded by fibres of sympathetic nerves, very much like ivy running round a tree. Arteries convey blood from the left ventricle of the heart to the various parts of the body. These vessels were named arteries from the belief entertained by the ancients that they contained air. Galen was the first to show that during life they contained blood.

\section{Distribution of the Arteries.}

The distribution of the systemic arteries is like a highly ramified tree, the common trunk of which, formed by the aorta, commences at the left ventricle of the heart, while the smallest ramifications extend to the peripheral parts of the body and contained organs.

Arteries are found in all parts of the body except in the hair, nails, epidermis, cartilages, and cornea of the eye. The larger ones usually occupy the most protected situations, running in the limbs, for instance, along the flexor side, where they are less likely to get strained or injured. A branch of an artery is smaller than the trunk from which it arises, but if an artery divides into two branches the combined sectional area of the two vessels is, in nearly every instance, somewhat greater than that of the trunk; and the combined sectional area of all the arterial branches greatly exceeds that of the aorta. So that the artesies, collectively, may be regarded as a cone, the apex of which corresponds 
to the aorta and the base of the capillary system. The arteries in their distribution communicate with one another, forming what are called anastomoses, and these communications are very free between the large as well as between the smaller branches. The anastomoses between trunks of equal size are found where great activity of the circulation is requisite, as in the brain for instance. The smaller branches of arteries anastomose more frequently than the larger, and between the smallest twigs these anastomoses become so numerous as to constitute a close network that permeates nearly every tissue of the body. The arteries are dense in structure, of considerable strength, highly elastic, and when divided they preserve, although empty, their cylindrical form.

\section{Degenerative Stages of Arteries.}

All the arteries and, most of all, the aorta, are liable to a degenerative process known as artero-sclerosis or athero-sclerosis. This condition is of the greatest clinical importance, especially in general anæsthesia. It is essentially a senile change, although it may begin at any age and is predisposed to by kidney disease, gout, diabetes mellitus, lead-poisoning, and a number of other morbid states, and results in the replacement of the arterial elastic tissue by fibrous tissue. Its chief ill-effects are two. In the first place, it is associated with a permanent and often considerable rise in the arterial blood-pressure, entailing a corresponding hypertrophy of the heart; in the second place, it weakens the vessel walls, rendering them liable to rupture, while, at the same time, it is apt to lessen the calibre of the affected vessels.

\section{The Pulse and what it reveals.}

The most characteristic feature of the arteries, from a dental point of view, is the pulse. It is the response of the arterial wall to the changes in lateral pressure caused by each heart-beat. The pulse is distinctly felt in the radial artery in the wrist just below the thumb on the inner side of the continuation of the palm of the hand. It is necessary in feeling a pulse to note the following points :

I. Its frequency - that is, the number of pulse beats per ininute. This gives the rate of the heart-beats.

2. Its strength: whether it is a strong, bounding pulse or a feeble beat. This indicates the force with which the heart is beating. 
3. Its regularity or irregularity; irregularity may occur owing to irregular heart's action either in force or rhythm.

4. Its tension or fulness-that is, the force necessary to stop it. This gives an indication of the state of the arterial walls as to their elasticity and peripheral resistance.

The pulse is caused by a wave of force which passes along the column of blood in the artery, due to a single contraction of the heart, so that each pulsation represents a heart-beat. Each ventricular contraction represents $3 \frac{1}{2}$ to $4 \frac{1}{2}$ foot-pounds. In twenty-four hours this is estimated to equal more than I2o foottons. Arterial tension is caused by the blood being forced into the arteries at considerable pressure. The arterial walls being very elastic permits the blood to be kept under elastic compression within them, so that when an artery is cut the blood spurts from it in a jet. The tension, however, is modified by the rate of the heart-beats, vasomotor nerve changes, the amount of the blood in the system, also the motion of the chest walls in breathing. The blood speeds through at the rate of about 35 feet per second.

Physicians in all ages have very properly attached considerable importance to the rate and force at which the circulation is carried on. As a measure of these conditions appeal is usually made to the pulse, as felt by the finger placed over the artery of the wrist. The pulse may be examined in any part where an artery is close to the surface so that its throb can be plainly felt. For general anæsthesia the pulse just in front of the ear on the face is a convenient one, and also one near the ramus of the jaw along its lower edge, but for ordinary examination the best one is at the wrist. In feeling the pulse we must be very careful not to flurry the patient or it will quicken the action of the heart and render our observations valueless. We should see that there is no pressure on the artery in any part of its course by tight sleeves or other article of dress. By throwing the shoulders well back it is possible to stop the pulse at the wrist. Malingerers sometimes manage to deceive the medical man in this manner.

\section{Practical Observation of the Pulse.}

The usual way of feeling the pulse is to place the three fingers just above the root of the thumb and the joint of wrist with your thumb on the opposite side so as to regulate the pressure. Its frequency may be measured by the seconds hand of a watch, 
but considerable practice is required to detect and appreciate its peculiar characteristics as indicative of various phases of disease: its rhythm, fulness, or softness; whether it is strong and bounding, forcing the fingers almost away from its surface; or hard, small, and wiry, like the vibrations of a string; whether, also, it is intermittent, striking a few beats and then apparently stopping one or two beats; also whether the pulsations flowing into each other are small and almost imperceptible. The information obtained by examining the pulse is often of the most interesting kind.

In disease the pulse may acquire a degree of frequency scarcely calculable to the touch, a rate of from I5o to 200 per minute being in some cases recorded. A rapid pulse, strong, full, and hard, indicates inflammation or fever; but if small and very rapid it points to a state of great debility. In apoplexy cr when fainting is impending, or in certain affections of the heart, the pulse may be very slow. Irregularity of the pulse is a condition which, as a rule, is full of meaning and interest. This condition is, curiously enough, natural to some people, and when they get ill with fever the pulse sometimes becomes quite regular. Irregularity of the pulse may be dependent on a number of very different conditions; it may be caused by disease within the head, or by disease of the heart, or it may be the result of simple disorder of the stomach, or of general debility. It does not follow because it is irregular that there is any serious danger. Smoking in excess or too generous use of stimulants may cause it.

Again, a pulse may be intermittent. When the motions of the artery are unequal in number and force, a few beats being from time to time more rapid and feeble than the rest, we say the pulse is irregular; but when from time to time a pulsation is entirely left out, we say it is intermittent. Frequently the intermission is perfectly regular, a pulsation being missed every fourth, tenth, or twentieth beat. When the intermissions are frequent, say every four or six beats, they are more likely to be regular. Intermittency of the pulse may be due to many diseases, but it is nct always of grave importance, for many trivial causes may produce it. It is rare in young children, but after middle age it is not at all uncommon. In some cases it is habitual, in others occasional only, and induced by some temporary constitutional derangement. Tobacco, tea, and stimulants are often responsible for this condition, or it may have a serious significance of the heart's action, brought about by 
rheumatic fever or other serious complications. Another important quality of the pulse is what is called hardness or incompressibility. We find that we can scarcely abolish the pulsation by any degree of pressure; the blood still forces its way through the artery beneath our fingers. Sometimes it is felt to strike a large portion of the finger, and we then say it is hard and full. When it strikes a very narrow portion of the surface of the finger, it is compared to a thread, and is a small pulse. A full pulse occurs in people who are plethoric, and also in the early stages of acute disease. A weak pulse denotes impoverished blood and an enfeebled condition of the system.

\section{Capillaries or Mesh formed by the Endings of Arteries and the Beginnings of Veins.}

The arterioles are the very fine and numerous endings of the arteries, which pass into capillary bloodvessels and are as fine as hairs, when they almost disappear and again become larger to form the capillaries that are the commencement of the veins which carry the blood back to the heart.

\section{TABLES WITH REFERENCE TO THE PULSE.}

\section{Frequency of Pulse.}

At birth $\quad \ldots \quad$. I I 30 to I50 times per minute.

First year .. .. I00 to I30 ,, ,

Seventh year .. 72 to 90 ," ,

Fifteenth year .. 80 to 85 ,, ,

Thirty-fifth year .. 69 to 75 ," ,

Seventieth year .. 50 to 60 ," ,

\section{FrequenCy OF RESPIRATION.}

First year .. $\quad$. 35 times per minute.

Second year .. $25 \quad$, ,

Fifteenth year .. 20 ", "

Above twenty .. I8 ,, ,.

Temperature of the Body.

Normal _ . . $97_{2}^{10}$ to $982^{\circ}$ Falırenheit .

Feverishness .. $99^{\circ}$ to $\mathrm{IOO}^{\circ}$,"

Slight fever .. .. $\operatorname{IOO}^{\circ}$ to IOI $^{\circ} \quad$,,

Moderate fever . IO $2^{\circ}$ to $\mathrm{IO}^{\circ}{ }^{\circ}$ ".

High fever .. . . $104^{\circ}$ to $105^{\circ}$,"

Intense fever $\quad 105^{\circ} \quad$," 


\section{Temperature in Relation to Pulse.}

Temperature of $98^{\circ} \mathrm{Fah}$. corresponds to pulse of 60

\begin{tabular}{|c|c|c|c|c|}
\hline "' & $99^{\circ}$ & "' & ", & $"$, \\
\hline ", & $\mathrm{IOO}^{\circ}$ & ", & ," & $"$, \\
\hline , & IOI $^{\circ}$ & ", & ," & "' \\
\hline$"$ & $\mathrm{IO} 2^{\circ}$ & ," & ", & ", \\
\hline " & $103^{\circ}$ & ", & ," & ," \\
\hline ", & $104^{\circ}$ & $"$ & "' & "' \\
\hline ,", & $105^{\circ}$ & " & ", & "' \\
\hline & $106^{\circ}$ & .. & " & ," \\
\hline
\end{tabular}




\title{
CHAPTER VI
}

\section{THE CIRCULATORY SYSTEM-(Continued)}

\author{
VEINS, BLOOD, BLOOD PRESSURE AND GASEOUS INTERCHANGE \\ WITH RELATION TO ASPHYXIA.
}

\section{Characteristics of Veins.}

THE veins of the human body are thin, ramifying, elastic tubes arising in the extremities of the body and proceeding by a more or less direct course to the heart, to which they carry back the blood sent forth by the arteries and transferred to them by the capillaries connecting the two kinds of vessels. They fall under three great divisions:

The Pulmonary.

The Systemic.

The Portal System.

The pulmonary veins consist of four short venous trunks, which carry the red blood back from the lungs to the left side of the heart, and which are found two on each side in the root of the corresponding lung.

The systemic veins arise by small branches which receive the blood from the capillaries throughout the body uniting to form larger vessels, and then two large venous trunks called the superior and inferior venæ cavæ, which finally enter the right auricle of the heart, into which the coronary veins also conduct the blood which nourishes that organ itself. These systemic veins are naturally divided into two groups, according to the channel by which they enter the heart. The veins of the head, the neck, the upper limbs, the spine, the heart, and part of the walls of the thorax and abdomen, make their entrance into the right auricle by the superior vena cava, while those of the lower parts of the body and the abdominal viscera do so by the inferior vena cava.

The veins of the portal system bring back the blood from the stomach, the intestines, the spleen, and the pancreas; then, joining, 
they form the great portal vein, which enters and ramifies in the surface of the liver after the manner of an artery, before finally entering the heart by the inferior vena cava. The anastomoses or branches leading to other veins, in a similar way to those described with reference to arteries, are much larger in veins than those of arteries. In many parts of the body there are two sets, one superior and the other more deeply seated, with frequent communications between the two. Some veins possess valves, while others are destitute of them. The walls of the veins are thinner than those of the arteries, but the veins themselves are less elastic. Their total capacity is much greater than that of the arteries, so much so that the veins alone can hold the volume of the blood which in life is distributed over both arteries and veins. While there is a considerable pressure even in the smaller and a greater one in the larger arteries, the pressure in the veins is greatest in those of smaller bore, and even in them it is but slight, so that while a pulse is present in arteries it is, as a rule, absent in veins. The velocity of the blood in the veins is least in those of smaller diameter and greatest in the larger trunks, which is the reverse of the rule in arteries.

The venous system begins in smaller vessels, which are slightly larger than the capillaries from which they spring. These vessels are gathered up into larger and larger trunks until they terminate (as regards the systemic circulation) in the two great veins called the venæ cavæ and the corcnary veins which enter the right auricle, and (as regards the pulmonary circulation) in four great pulmonary veins which enter the left auricle from the lungs. The total capacity of the veins diminishes as they approach the heart, but as a rule their capacity is two or three times that of the corresponding arteries. The pulmonary veins, however, are an exception to this rule, as they do not exceed in capacity the pulmonary arteries. The veins do not keep their shape when empty like the arteries, and are found after death in a more or less collapsed condition owing to their lack of elasticity. They are usually distributed in a superficial and also a deep set, which communicate frequently in their course. In structure, the coats of veins bear a general resemblance to those of arteries. Thus they possess an outer, middle, and internal coat; but there is very much less muscular tissue in the veins than there is in the arteries, and, indeed, the veins of bones, and of the central nervous system and its membranes, possess no tissue of a muscular nature. 


\section{Valves in the Veins to prevent Back Pressure.}

There is, however, one main distinction between arteries and veins, which is the presence of valves in the latter vessels. The general construction of these valves is similar to that of the semilunar valves of the aorta and pulmonary artery, but their free margins are turned in the opposite direction - that is, towards the heart, so as to prevent any movement of blood backward. They are commonly placed in pairs in different veins, but almost uniformly in each. In the smaller veins single valves are often met with, and three or four are sometimes placed together or near one ancther in the largest veins, such as the subclavian in the shoulders, at their juncticn with the jugular veins. The situation of these valves may be seen in the superficial veins of the forearm, and are readily discovered by pressing along their surface in the direction opposite to the venous current, which is from the elbow to the wrist, when little swellings appear in the position of each pair of valves. These swellings at once disappear when the pressure is removed.

Valves are not equally numerous in all veins, and in many they are absent altogether. The blood current in the veins is maintained primarily by the foree behind it, which is the bloodpressure transmitted from the heart and arteries; but very effectual assistance to the flow is afforded by the action of the muscles capable of pressing on the veins with valves, as well as by the suction action of the heart, and also by the aspiratory action of the thorax. The brief outline given shows the difference between arteries and veins in their structure and function. We shall now pass on to indicate the nature of the liquid which circulates through the arterial and venous vessels which constitute the circulatory system.

\section{Description of the Blood.}

The blood is an opaque, rather viseid fluid, of a bright red or scarlet colour when it flows from the arteries, and of a dark red or purple colour when it flows from the veins. It is salt to the taste, and has a peculiar faint odour and an alkaline reaction. Its temperature is generally about $99^{\circ}$ Fah., though varying slightly in different parts of the body. 


\section{Plasma and Corpuscles.}

Blood consists of a faintly yellow fluid called the plasma or liquor sanguinis, in which are suspended numerous minute particles, termed "blood corpuscles," the majority of which are coloured, and give to the blood its red tint. If a drop of blood be placed in a thin layer on a glass slide and examined under the microscope, a number of the corpuscles will be seen floating in the clear fluid plasma.

The blood corpuscles are chiefly of three kinds:

I. Coloured corpuscles or erythrocytes.

2. Colourless corpuscles or leucocytes.

3. Blood platelets, which are of minor importance.

\section{Erythrocytes, or Red Corpuscles.}

Coloured or red corpuscles, whose scientific name is erytlirocytes, when examined under the microscope are seen to be circular discs, biconcave in profile. It is to the aggregation of the red corpuscles that the blood owes its red hue, although when examined by transmitted light their colour appears to be only a faint reddish yellow. They vary slightly in size, even in the same drop of blood, but the average diameter of each is $\frac{1}{3200}$ of an inch, and the thickness about $\frac{1}{1 \cdot 000}$ of an inch or nearly a quarter of the diameter. Besides these there are found certain smaller corpuscles of about one-half or one-third of the size just indicated; these are termed "microcytes" and are very scarce in normal blood. In diseased conditions, such as anæmia, however, they are more numerous. The number of red corpuscles in the blood is cnormous-between $4,000,000$ and $5,000,000$ are contained in a cubic centimetre.

\section{Hæmoglobin and its Importance.}

Each corpuscle consists of a colcurless, elastic sponge-work or stroma, condensed at the periphery to form an investing membrane; uniformly diffused throughout the stroma are the coloured fluid contents. The stroma is composed mainly of nucleo-protein and of the fatty substances, lecithin and cholesterin, while the coloured material consists chiefly of the respiratory protein called hæmoglobin, which is a compound protein composed of an iron-. containing body called hæmatin, and of globin, which is a protein 
belonging to the globulin group. Hæmoglobin has a great affinity for oxygen, and when removed from the body it crystallizes readily under certain circumstances. It is very soluble in water.

Even when examined in very thin layers, blood is opaque, on account of the different refractive powers possessed by its two constituents-viz., the plasma and the corpuscles. On treatment with ether, water, and other reagents, however, it becomes transparent and assumes a lake colour, because this chemical treatment discharges or releases the colcuring matter of the corpuscles intc the plasma.

The blood is characterized by its ability to take up certain gases which alter the colour of it. This is due to the hæmoglobin contained in the corpuscles having varying amounts of oxygen. The carbonic oxide capacity is the same as the oxygen capacity, as is shown by repeated experiments. The pigment is by far the most abundant and important of the constituents of the red corpuscles, and is a compound of protein with the iron-containing pigment called hæmatin. It exists in the blood in two conditions: in arterial blood it is loosely combined with oxygen, is of a bright-red colour, and is called oxy-hæmoglobin. The other condition is the deoxygenated or reduced hæmoglobin, better simply called hæmoglobin. This is found in the blood after asphyxia. It also occurs in all venous blood - that is, blood which is returning to the heart after it has supplied the tissues with oxygen. Venous blood, however, always contains a considerable quantity of oxyhæmoglobin also. Hæmoglobin is the oxygen carrier of the body, and it may be called a respiratory pigment. Crystal oxyhæmoglobin may be obtained with readiness from the blood of such animals as the rat, guinea-pig, or dog, and with difficulty from other animals such as man, ape, and most of the common mammals:

I. Mix a drop of defibrinated blood of the rat, etc., on a slide with a drop of water; put on a cover glass; in a few minutes the corpuscles are rendered colourless, then the oxy-hæmoglobin crystallizes out from the solution so formed.

2. Microscopical specimens may also be made by Stein's method, which consists in using Canada balsam instead of water in the foregoing experiment.

3. On a larger scale, crystals may be obtained by mixing the 
blood with one-sixteenth of its volume of ether: the corpuscles dissolve and the blood assumes a laky appearance. After a period varying from a few minutes to days abundant crystals are deposited.

4. Crystals of hæmoglobin can also be obtained by carrying out the crystallization in an atmosphere free from oxygen. Upon adding an acid or alkali to hæmoglobin it is broken up into two parts: a brown pigment called hæmatin, and a protein called globin. These substances are further analyzed by the chemist into other substances.

\section{Leucocytes, or White Corpuscles.}

The colourless corpuscles, whose scientific name is leucocytes, are of various sizes, some no larger, others smaller, than the red corpuscles. In human blood, however, the majority are rather larger than the red corpuscles, and measure about $\frac{1}{2000}$ to $\frac{1}{2500}$ of an inch in diameter. On the average from 10,000 to 12,000 leucocytes are found in each cubic millimetre of blood. They consist of minute masses of nucleated protoplasm, and exhibit several varieties, which are differentiated from each other chiefly by the occurrence or non-occurrence of granules in their protoplasm, and by the staining reactions of these granules when present. These various forms are all capable of analysis by the chemist and are classified accordingly.

The colourless corpuscles are very various in shape in living blood, because many of them have the power of constantly changing their form by protruding finger-shaped or filamentous processes of their substance by which they move, and take up granules from the surrounding medium. In locomotion the corpuscle pushes out a process of its substance, called a pseudopodium, and then shifts the rest of its body into it. In the same way, when any granule or particle comes in its way the corpuscle wraps a pseudopodium around it and then withdraws the pseudopodium with the contained particle into its own substance. By these peculiar properties (termed " amœboid movements ") the cells have the power of wandering or emigrating from the bloodvessels by penetrating their walls, and thus finding their way into the extra-vascular spaces. A chemical investigation of the protoplasm of the leucocytes shows the presence of nucleoprotein and of globulin. The occurrence of small amounts of fat and glycerine may also be demonstrated. The blood platelets are discoid or irregularly shaped, colourless, refractile bodies, 
much smaller than the red cells of corpuscles. Considerable discussion has arisen as to their significance. Recent observers have shown that under the action of certain stains the centrallysituated portion of the blood platelet takes on the appearance suggestive of a nucleus. In spite of this, and of the fact that they have been observed in the bloodvessels during life, there is still a tendency to regard them as products of disintegration of the white cells or leucocytes, or as precipitates possibly of nucleoprotein, and nct as living elements of the blood.

\section{Importance of Leucocytes as Patrols of the System.}

The most important outcome of the amœboid movement of the colourless corpuscles is their power of ingesting or feeding on foreign particles such as bacteria, which they engulf or digest. This is called phagocytosis, and plays an incalculable part in the removal of all waste products of the body, and the bacteria and products of disease. We shall refer to this point in another chapter.

\section{The Plasma, or Liquid Part of the Blood.}

The plasma, or liquor sanguinis, which is the fluid portion of the blood in which the red and white corpuscles move, is as interesting and important as these cells. It has a yellowish tint, is alkaline in reaction, and has a specific gravity of $\mathrm{I}^{\circ} \mathrm{O} 28$. It contains in solution about so per cent. of solids, of which four-fifths are protein in nature, the remainder being chemical salts, chiefly chlorides, phosphates, and sulphates of the alkaline metals; carbohydrates, chiefly sugar; fats and soaps; cholesterin, urea, and other nitrogenous substances. The proteins are three in number:

Serum albumen.

Serum globulin.

Fibrinogen.

Fibrinogen is a body from which the substance fibrin, which plays such an important part in the clotting or coagulation of the blood, is derived. In addition there are represented several substances of very great importance in connection with the subject of immunity or resistance to bacterial development. The liquid part of the plasma is called serum, and is the yellowish fluid which separates from the blood after the coagulation of the fibrin. 


\section{Lymph and its Importance.}

There is another fluid which, although mixing with the blood, yet must be considered as being a separate solution. We refer to what is called lymph. Lymph is a transparent, colourless or slightly yellow fluid which is conveyed by a set of vessels named lymphatics into the blood. These vessels arise in nearly all parts of the body as lymph capillaries. They take up the blood plasma which has exuded from the blood capillaries for the nourishment of the tissue elements and return it into the veins. The greater number of these lymphatics empty themselves into one main duct, called the thoracic duct, which passes upwards along the front of the vertebral column and opens into the large veins on the left side of the root of the neck. The remainder empty themselves into a smaller duct, which ends in the corresponding vein on the right side of the neck.

Lymph is a watery fluid which closely resembles the blood plasma, but is more dilute, containing only about 5 per cent. of proteins and I per cent. of salts and extractives. When examined under the microscope, lymphocytes or cells similar to leucocytes are found floating in the transparent fluid; they are always increased in number after the passage of the lymph through lymphoid tissue, as in lymphatic glands. Lymph varies greatly in composition in different parts of the body. The lymph leaving the liver has the greatest supply of proteins and has the highest specific gravity. The intestinal lymph, called chyle, is intermediate in composition, and contains in addition the fat absorbed by the lacteals in the coatings of the bowels, which gives it a milky appearance.

Having endeavoured to place a clear outline before our readers of the separate parts of the blood, we will now pass on to a consideration of the vital functions of the blood, with the object of showing what is taking place continuously by its circulation, and also what takes place in its endeavours to combat the conditions of disease.

This will be best considered under three divisions, from a dental point of view, as enumerated below, although it must be clearly understood that from a general and comprehensive standpoint the whole subject of the blood circulation is almost endless in its chemical and dynamic details.

I. Characteristics of the circulation of the blood.

2. Immunity of the blood.

3. Coagulation of the blood. 
The consideration of these three great divisions of our present subject is of paramount importance to the dental practitioner, so that while he will always have cases which cause anxiety, yet it must be a source of interest and satisfaction to know the factors which will be in favour of health and success in the course of his many methods of dealing with diseased dental conditions. We have here reached what the writer considers the vital factor underlying all our work-namely, the functions of the blood in maintaining health and combating disease. If this is applicable to the surgeon and physician it must apply also to our own case, where we are constantly involving the blood in septic conditions of the mouth, and especially where the vessels are of appreciable size, and also being within twelve inches of the great veins which immediately enter the heart, each inch leading towards that organ becomes correspondingly larger. The proximity of the veins of the mouth to the central circulatory system must perforce demand great care on the part of the practitioner both as to drugs which he uses in injections, and also as to precautions to be taken for antiseptic treatment. Whether we attempt to extract a tooth or to apply root treatment or in filling teeth, and even in any operation in the mouth, there is always present the possibility of inflammatory conditions arising with the attendant sequelæ of pus with its dangers; so that a great deal depends upon an intelligent estimate of the nature of the vital stream.

In considering some of the important characteristics of the blood stream we refer rather to the vital changes from cause to effect in:

a. Blood-pressure.

$b$. Interchange of gases.

c. The vaso-nervous system.

The circulation of the blood depends upon the existence of different degrees of pressure in different parts of the circulatory system. There is a diminution of pressure from the heart onwards through the arteries, capillaries, and veins, back to the heart again. The contraction of the heart is the primary propelling force, and the increase of pressure which is thus communicated to the blood it contains causes that blood to enter the arteries. The arterial blood-pressure is higher than that in the capillaries, and the capillary pressure is higher than that in the veins; the venous pressure gradually falls as it approaches the heart. It is lowest of all in the heart cavities during diastole or dilatation. Fluid 
moves in the direction of lower pressure, hence the flow of blood is from the heart through the vessels to the heart again. Nature has provided for this by gradually enlarging the capacity of the bloodvessels as they proceed in the arteries until the bed of the stream is thus becoming greater, until we reach the capillaries when the total bed is suddenly and enormously increased, being several hundred times greater than that of the aorta from which they all ultimately spring. In the case of the veins, the same is true in the reverse direction; the sectional area of a vein is less than that of the total sectional area of its tributaries. Hence as we approach the heart the total bed of the stream is becoming smaller, but is never as small as in the corresponding arteries. A vein is at least twice the size of, and often larger than, the corresponding artery. The pulse is one of the main characters of the arterial flow.

In connection with the variation of the capacity of the bed, we have to consider the question of resistance. The main resistance to the passage of blood through the tissues is situated in the arterioles and not in the capillaries. This is usually spoken of as the peripheral resistance, and is variable according to the calibre of the arterioles and their muscular tissues being under the control of vaso-motor nerves. The main resistance is in the arterioles and not in the capillaries, for the following reasons: each individual capillary is small, and its resistance, therefore, great, but their number is so immense and the total bed so large that the resultant resistance offered is comparatively small.

Again, the velocity of the stream has to be taken into account, which is greatest in the aorta, lessens in the small arteries, and becomes slowest of all in the capillaries, which may be regarded as a vast lake into which the arterial blood flows. The bloodpressure, therefore, is highest when leaving the heart, and becomes less as the arteries proceed and branch out. It again falls in the capillaries and the veins, until in the large veins near the heart it is negative. Velocity is different; the velocity, like the pressure, falls from the arteries to the capillaries, but, unlike it, rises again in the veins.

The movement of the lungs and chest walls within the airtight thorax exerts marked pressure on the large arteries and veins contained within it. The main effect of respiration on the circulation is that arterial pressure rises with inspiration and falls 
with expiration, but is not quite synchronous; in each case it is a little behind time. The heart beats more rapidly during the rise of blood-pressure than during the fall. The rising and falling, too, of the diaphragm exert pressure also upon the abdominal veins. The pressure outside the heart and thoracic vessels is correspondingly diminished during expiration, so that the chest movements affect the circulation, and forms the thoracic pump. Gravity has an effect also on the circulation, but is overcome largely by the pumping action of the chest, lungs, and diaphragm. Nevertheless, if the strain on the heart is severe at any time it is well for the patient to assume the horizontal position, as the vertical pressure of the circulation is definitely relieved.

\section{The Interchange of Oxygen with Carbonic Acid Gas.}

The next point for us to observe is that of the process whereby the gases of expiration and inspiration are interchanged. In this process the action of the lungs plays a most important and indeed a vital part. The process of breathing, by which oxygen is taken into the lungs by inspiration and carbonic acid gas is expelled by expiration, is going on without intermission, whereby carbonic acid gas is given off from the blood, oxygen taking its place. When respiration is interfered with asphyxia takes place under ordinary circumstances. The action of respiration exposes the blood to the air, and, by mutual diffusion, the two actions of oxygenating the blood and freeing it of carbonic acid are accomplished by the same act, thus resembling the chemical processes of nature known as endosmosis and exosmosis. The process as applied to the fluids and gases of the body is termed osmosis. The action of gaseous interchange involved with the process of respiration is owing to reflex nervous action and not dependent on the will, as the same process goes on in sleep and in other unconscious states. The lungs themselves are almost passive in the process. From every loo cubic centimetres of arterial blood rather more than 20 cubic centimetres of oxygen can be removed by the air pump. Nearly all of this oxygen is chemically combined with hæmoglobin. The amount, though, in actual solution is small, being $0^{\circ} 7$ centimetre for every 100 centimetres. Hæmoglobin owes its value as a respiratory pigment to these principal facts: Firstly, it can unite with a large quantity of oxygen; in fact it can carry about thirty times as much to the 
tissues as plasma would under the same circumstances. Secondly, the interaction between hæmoglobin and oxygen is a reversible one: the two unite in the lungs where the pressure is high, but when oxygen is absent or at a low pressure, as in the tissues, the hæmoglobin parts with its store of oxygen.

If blood is divided into its two separate parts-plasma and corpuscles-it will be found that both yield carbonic acid, but the yield from the plasma is the greater.

One hundred volumes of venous blood contains about 46 volumes of carbonic acid gas. But, although arterial blood contains 20 per cent. of oxygen gas, and venous blood contains 46 per cent. of carbonic acid gas, yet this by no means represents the actual amount of these gases inspired or respired at each movement of breathing. These gases are always present in the blood together at the same time, and only a small proportion is interchanged.

Arterial blood contains:

Oxygen, 20 per cent.

Carbonic acid gas, 40 per cent.

Nitrogen, 2 per cent.

Venous blood contains:

Oxygen, 8 to I2 per cent.

Carbonic acid gas, 46 to 50 per cent.

Nitrogen, I to 2 per cent.

It will be noticed that the amount of nitrogen in the blood is small in amount. It has no physiological significance, and is nearly the same in both varieties of blood. The important distinction between arterial and venous blood is in the other two gases, and, as the table shows, on the average every roo cubic centimetres of venous blood which passes through the lungs gains 8 cubic centimetres of oxygen and loses 6 of carbonic acid gas.

\section{Osmosis or Diffusion in Gaseous Interchange.}

The principle of the gaseous interchange which occurs in the blood while passing through the lungs is called diffusion or osmosis. When applied to gases it means the passing of one gas into the space occupied by another. When applied to liquids it means that when two liquids that are capable of mixing are put into contact with a thin separating medium of parchment between them they gradually diffuse one into the other, notwithstanding 
the action of gravity. Thus, if a vessel containing a solution of common salt be carefully placed with its mouth covered in a vessel containing water, the water being sufficiently deep to cover the vessel of salt and water, and if the cover be removed from that vessel, in time the salt and water solution will diffuse out into the larger vessel, and the water into the smaller vessel, until both fluids are of equal density. With gaseous interchange in the lungs we have the same principle of a thin membrane separating two chemicals, but it is gaseous with a liquid vehicle. There are, accompanying this form of osmosis or diffusion, many complicated factors which are conformable to physical, physiological, anatomical, and chemical laws, which are thoroughly understood by authorities on the blood.

\section{The Control of the Capacity of Arterioles by Vasomotor Nerves.}

Another characteristic of the blood circulation, and which is of paramount importance, is the control of its flow by the condition of the bloodvessels as to their carrying capacity under various conditions of the general system or local parts. The carrying capacity of the arteries and veins is controlled by what is called the vascular nervous system.

Arteries are supplied with nerves which are derived from the sympathetic nervous system. They form intricate branching centres, called plexuses, upon the surfaces of the larger trunks, and run along the smaller arteries as single filaments or bundles of filaments, which twist around the vessel and unite with each other in a plexiform or network manner. The branches derived from these plexuses penetrate the external coat of arteries and are distributed principally to the muscular tissue of the middle coat, and thus regulate, by causing the contraction and relaxation of this tissue, the amount of blood sent to any part.

Veins are also supplied with nerves, but in much less abundance.

The vascular nervous system is termed the vasomotor system and consists of the vasomotor centre, which is situated in the base of the brain, called the medulla, also of certain subsidiary vasomotor centres in the spinal cord, and of vasomotor nerves, which are of two kinds:

I. Vaso-constrictor nerves, which cause constriction or contraction of bloodvessels.

2. Vaso-dilator nerves, which cause dilation. 


\section{Peripheral Resistance in the Arterioles of Great Importance.}

The nerves exert their most important action in the vessels which contain relatively the greatest amount of muscular tissue, viz., the smaller arteries or arterioles. Under ordinary circumstances the arterioles are maintained in a state of moderate or tonic contraction, and this constitutes the normal peripheral resistance, the use of which is to keep up the arterial pressure, and which must be high enough to force the blood through the capillaries and veins in a continuous stream back to the heart. Another function which is served by this muscular tissue is to regulate the amount of blood which flows through the capillaries of any organ, locally, in proportion to its needs. The vasomotor centre can be excited directly by electric induction currents; the result is an increase of arterial blood-pressure, owing to an increase of the contraction of the peripheral arterioles. It can also be excited by the action of poisons in the blood which circulate through it. Strophanthus or digitalis causes a marked rise of general arterial pressure, due to the constriction of the peripheral vessels brought about by impulses from the centre. It is also excited by the condition of venous blood, as in asphyxia. The rise of blood-pressure which occurs during the first part of asphyxia is due to the constriction of peripheral vessels, and the fall of pressure during the last stage of asphyxia is largely due to heart failure.

The vasomotor nervous system is influenced to some extent by conditions of the cerebrum, some emotions, such as fear, causing pallor by vaso-constriction, and others causing blushing by vaso-dilatation. It is almost impossible to over-estimate the importance of the study of vasomotor phenomena as a means of explaining certain pathological conditions. In certain conditions, for instance, which lead to angina pectoris, the pain in the heart is, in part, due to its being unable to overcome an immense peripheral resistance, and the condition is relieved by the administration of such drugs as amyl-nitrite or nitro-glycerine, which relax the vessels and cause universal blushing.

\section{Asphyxia and its Causes.}

The condition of asphyxia is a most important one to be taken into consideration by every dental practitioner when referring to the vasomotor nervous system. 
Asphyxia may be produced in various ways:

I. By the prevention of the due entry of oxygen into the blood, either by direct obstruction of the trachea or other parts of the respiratory passage.

2. By introducing, instead of ordinary air, a gas devoid of oxygen.

3. By interference with the due interchange of gases between the air and the blood.

\section{Stages of Asphyxia.}

The symptoms of asphyxia may be roughly divided into three stages:

r. Exaggerated breathing.

2. Convulsions.

3. Exhaustion.

In the first stage breathing becomes more rapid and deeper than usual, inspiration at first being especially exaggerated and prolonged. The muscles of extraordinary respiration are called into action, and the effort to respire is laboured and painful. This is soon followed by a similar increase in the expiratory efforts, which become excessively prolonged, being aided by all the muscles of extraordinary expiration. During this stage, which lasts a varying time from a minute upwards according as the deprivation of oxygen is sudden or gradual, the lips become blue, eyes are prominent, and the expression intensely anxious. The prolonged respirations are accompanied by a distinctly audible sound, and the muscles attached to the chest stand out as distinct cords. This stage includes the two conditions hyperpnœa, or excessive breathing, and dyspnœa, or difficult breathing, which follows later. It is due to the increasingly powerful stimulation of the respiratory nervous centre by the increasingly venous blood.

In the second stage, which is not marked by any distinct line of demarcation from the first, the violent expiratory efforts become convulsive, and then give way to general convulsions, which arise from the further stimulation of the centres in the brain and spinal cord by the venous blood. Spasms of the muscles of the body occur in general, and not of the respiratory muscles only. The convulsive stage is a short one, and lasts less than a minute. 
The third stage is that of exhaustion. In it the respirations all but cease, the convulsive spasms give way to flaccidity of the muscles, there is insensibility, the conjunctivæ of the eyes are insensitive, and the pupils are widely dilated. Every now and then a prolonged sighing inspiration takes place, at longer and longer intervals, until breathing ceases altogether, and death ensues. During this stage the pulse is scarcely to be felt, but the heart may beat for some seconds after the respiration has stopped. The condition is due to the gradual paralysis of the nerve centres by the prolonged action of the venous blood. This stage may last three minutes and upwards. After death from asphyxia it is found in the great majority of cases that the right side of the heart, the pulmonary arteries, and the systemic veins are gorged with dark, almost black, blood, and the left side of the heart, the pulmonary veins, and the systemic arteries, are empty. The explanation of these appearances is thus summarized. When oxygenation ceases, venous blood at first passes freely through the lungs to the left heart, and so to the great arteries. Owing to the stimulation of the vasomotor centres by the venous blood the arterioles become constricted through the vaso-constrictor nerves. The arterial blood therefore rises and the left side of the heart becomes distended. The highly venous blocd passes through the arterioles, and, favoured by the laboured respiratory movements, arrives at the right side of the heart, which it fills and distends. The right side of the heart becomes feebler at the same time, because of its overloaded condition, and therefore unable to effectively discharge its blood through the pulmonary circuit in the lungs. Simultaneously the left ventricle is also becoming weakened, and therefore the suction action diminishes. In this way the blood is dammed back in the right heart and veins, and the left side of the heart, therefore, gets into the empty condition in which it is found after death.

In the first stages of asphyxia the arterial pressure rises above the normal; this is due to the constriction of the arterioles. The fall of pressure in the last stage is mainly due to heart failure. 
CHAPTER VII

\section{THE CIRCULATORY SYSTEM-(Continued)}

THE IMMUNITY OF THE BLOOD AND ITS COAGULATIVE PROPERTIES, WITH RELATION TO ASEPSIS AND H EMORRHAGE.

THE consideration of the remaining two great divisions of the vital functions of the blood is of no less importance to the dental operator than the preceding chapter, but we would, however, lay stress on the points before us as being the goal to which we have deliberately led our readers in the foregoing description of the circulation of the blood. The operations upon the teeth and the tissues surrounding them, when skilfully carried out, should maintain at least the normal degree of health which obtained when the patient presented himself. The object of the dental operator in all operations is to bring all diseased conditions up to the patient's normal state of health. We would also remark that no operator is justified in making the degree of inflammation worse than when he first found it, even if he does not succeed in making it better, but he must so master his work that the normal degree of health is reached by his selection of operative methods and suitable medical treatment.

Most operators take it as a matter of course that when they extract a tooth the fearful wound will take care of itself, and the same view of the operation is extended to thousands of teeth extracted, and yet they are enormously surprised, and in some cases mystified, by the fact of a case here and there causing some anxiety both to themselves and their patients on account of the tardiness of the socket to heal up. There may be considerable sloughing, and in some cases a decided disinclination to heal up, apart from asepsis. To take a rational estimate of these cases by comparison with the cases that heal up without any trouble at all we ought to reverse them, and the surprise and wonder should be expressed by the question, Why do these straightforward cases heal up so nicely? The operator should at all times regard all extraction operations as being on a level with 
root treatment, which would lead him to be ready for reverse developments and deal with them accordingly. The two operations of extraction and root treatment, though usually regarded as being wide apart in the degree of skill involved, are identical in their principles of maintaining or reaching the normal degree of health of the socket tissues. To put it clearly, we will assume that a patient presents himself with an abscessed tooth before two operators. One decides to extract the tooth, the other one undertakes to cure the abscess and save the tooth. The principle that is identical in the two operative methods is that the health of the tissues must be secured in either case, and in each method the abscess is dealt with in order to rid the socket of septic trouble. But the operator who extracts the tooth has much the easier task, because in removing the tooth he can at once reach the abscess, which he sometimes is apt to leave to itself with serious consequences. The second operator, who decides to treat the abscess in order to cure it, voluntarily undertakes all the risks because he realizes the factors that will help him, although his work may be tedious and difficult, yet he is confident of a ready response of the tissues to his treatment.

\section{The Factors underlying all Dental Operations.}

What, then, are the factors that help the dental operator in his work ? The definite answer to this vital question is:

I. The resistance of the blocd to bacterial invasion, indicated by the term immunity.

2. Coagulation of the blood, in order that the normal quantity in circulation shall not be seriously reduced through bleeding and pending repair of the bloodvessels.

Immunity of the blood is its power of resisting the development of infections, or morbid processes, in the body. This property is exercised by the presence of various chemical constituents such as agglutinins, lysins, opsonins, and antitoxins. The study of these substances can only be undertaken in suitable laboratories where the exacting precision of analysis may be verified and recorded. There are definite methods of investigation which produce tangible evidence of the existence of the factors that are involved in the resistance of the blood to the activities of bacteria. The instruments used include elaborate scientific equipment, and such instruments as the microscope and its 
never-ending adjustments for magnification and illumination, the spectroscope, with which the shades and colours of chemical substances can be detected and measured, and also elaborate electrical apparatus. So that there is no lack of evidence that the proofs of recognition of the substances examined are beyond doubt when verified and corroborated by several experts in these subjects.

Natural immunity of the blood, organs, and tissues of the body is carried out by their own inherent chemical constituents. Their action in combating particular bacteria may be increased by the inoculation of chemical substances, called antitoxins. They are employed in suitable strengths, and under proper conditions of administration, whereby their chemical and bacteriological activities are maintained for a long period of time. It can be acquired also by the chemical products of certain diseases whereby the blood is immune against a repeated attack of that disease, such as smallpox, or vaccination for the prevention of that disease, which has the same effect. This last method of inoculating anti-toxin is termed artificial.

It must be understood that the degree of immunity varies considerably in consequence of various factors. The importance of the discoveries regarding immunity becomes more valuable to us in proportion as we realize that the blood contains within itself the means whereby disease can be combated. The life of an organism is in its blood; the blood is the life, and keeps it in a positive condition. Any interference with its normal condition of health gives rise to inflammation, so that it may be regarded as the evidence of the action of the blood to cast out the germs of threatened disease. It is in reality a combat between the policemen contained in the blood and the invading bacterial enemy. In this way the body is protected by chemical defences against injury and disease.

The acid of the gastric juice in the stomach is a great protection against harmful bacteria being introduced with food. The acidity of urine has a marked effect upon bacterial growth within that secretion.

The leucocytes, or white corpuscles, called also phagocytes, destroy bacteria by feeding upon them (see Chapter VI.). Also the fluid part of the blood is often antagonistic to bacterial life. This fact was first discovered when bacteriologists experimented with blood serum as being a suitable medium in which to grow 
or cultivate bacteria. It was thought quite probable that it would be a natural soil for it, but it was found in some instances to have just the opposite effect.

The bactericidal powers of the blood, however, are destroyed by heating it for some time to $55^{\circ} \mathrm{C}$. The chemical substances contained in the serum are called bacteriolysins.

Besides the bactericidal powers of the blood serum there is also present its globulicidal power. This means that the blood serum of one animal has the power of dissolving the red corpuscles of another species. If the serum of one animal is injected into the blood-stream of another species the result is a destruction of its red corpuscles, which may be so excessive as to lead to the passing of the liberated hæmoglobin into the urine (called hæmoglobinuria).

The substances in the serum possessing this property are called hæmolysins, and although there is some doubt whether bacteriolysins and hæmolysins are identical, yet they are very closely related substances.

Normal blood possesses a certain quantity of substances which are inimical to the life of our bacterial foes, but when a person gets run down it is common knowledge that he is then more liable to catch anything. This coincides with a diminution in the bactericidal power of his blood. But even a healthy person has not an unlimited supply of bacteriolysin, and if the bacteria are sufficiently numerous he will fall a victim to the disease which they produce. Here, however, comes in the remarkable part of the defence. In the struggle he will produce more and more bacteriolysin, and if he gets well it means that the bacteria are finally vanquished, and his blood remains rich in the particular bacteriolysin he has produced, and so will render him immune for a time to further attacks from that particular species of bacterium. Each bacterium attacked in this way seems to cause the development of a specific antibody.

The blood has the means of combating our bacterial enemies in various ways. In some cases they are rendered immobile by agglutinins, in other cases they are killed by bacteriolysins. In other instances their toxins are neutralized by antitoxins, and in others, again, they are directly devoured by the phagocytes. Metchnikoff's view, which is shared by many eminent bacteriologists, is that phagocytosis, or the devouring function of the phagocytes or leucocytes, is the supreme method, and the others are 
auxiliary. The immunity of the blood is therefore a vital factor to the dental operator in the healing up of wounds and sockets after extractions, and this should be taken into serious consideration in dealing with weak patients, and also with mouths that present an extremely unhealthy appearance. In injecting alkaloids such as cocaine, etc., it must be borne in mind that they are protoplasmic poisons, and if the parts are already inflamed and diseased it cannot be expected that the injection of a tissue poison is going to help the healing of them. But providing the operator is aware of the condition of his patient he can, as a rule, help the case materially by using suitable antiseptic mouthwashes and bringing the patient back for treatment until the parts are healthy. The wonderful activity, however, of the periosteum may be counted upon, but as it is so delicate it must be helped by mild antiseptic and prophylactic treatment; in other words, get that organ healthy and it will then attend to the healthy repair of the bone, which is the main consideration in dental operations. The medical man can render valuable aid by administering suitable medicines and treatment systemically in bad cases.

\section{The Basic Principles of the Process of Coagulation.}

The third great division of the vital functions of the blood comes under the term " coagulation." This quality of the blood is equally of importance to the dental operator as the two we have already described. This is another instance where the body has a means of defence. In this case it is a protection against loss of blood, with its dire and calamitous consequences. There are some persons (fortunately few) who suffer from what is called " hæmorrhagic diathesis," which means that they bleed freely and profusely from the slightest scratch or cut, and their blood is so peculiar that there is the greatest difficulty in stopping its flow. This disease is fortunately of not very common occurrence. Thus patients and parents of children with this tendency should always inform the dentist of this fact if they are aware of it, in order that, as far as possible, it can be dealt with previous to the operation, so that the serious loss of blood may be prevented when the operation does take place. But there are very rare cases where no treatment whatever will be available. Such cases, when known beforehand, should be placed in the hands of a 
medical man for treatment before and after the operation. But the treatment of hæmorrhage after the extraction of teeth can usually be made successful if the principles of the coagulation of the blood are understood. It is not our intention in this chapter to deal with the practical treatment of hæmorrhage, but we will do so in the proper place in a future chapter.

When blood is drawn and allowed to stand it emits a " halitus" or exhalation, which has a faint smell. In three or four minutes a film overspreads the liquid, commencing at the circumference and gradually spreading to the centre. Two or three minutes later the lower part of the blood in contact with the vessel becomes solidified and then the whole mass, eight or nine minutes being needful for the whole process from first to last. In about fifteen or twenty minutes a thin serum begins to exude from it, which goes on for two or three days. This forms what we call a clot. At first it sets into a firm red jelly which soon contracts and squeezes out a straw-coloured fluid called serum. Under the microscope filaments or fine threads are seen forming a network throughout the fluid, many of them radiating from small clumps of blood platelets. These threads entangle the corpuscles, and in this way the clot is formed. But what actually takes place involves a very interesting chemical process revealing once more the never-ending resources of the chemical constituents of the blood. The threads are composed of a protein substance called fibrin, and the formation of this fibrin is the essential act of coagulation.

Fibrin is formed from the plasma, and may be obtained free from corpuscles when plasma is allowed to clot, the corpuscles having previously been removed by suitable methods. It may also be obtained by whipping fresh blood with a bunch of twigs. The fibrin adheres to the twigs and entangles but few corpuscles, and these may be removed by subsequent washing with water.

Serum is plasma minus the fibrin which it forms. The relation of plasma, serum, and clot, can be seen at a glance in the following scheme of the constituents of the blood:

Blood divides
into $\left\{\begin{array}{ll}\text { Plasma. } & \begin{array}{l}\text { Plasma } \\ \text { divides into }\end{array} \\ \text { Corpuscles. } & \text { Serum, } \\ \text { Fibrin }\end{array}\right\} \begin{aligned} & \text { Fibrin from the Plasma } \\ & \text { acting with the Cor- } \\ & \text { puscles form the Clot. }\end{aligned}$

It may be roughly stated that in Ioo parts of blood 60 to 65 parts consist of plasma and 30 to 35 parts of corpuscles-roughly about two parts of the former to one of the latter. 
In the act of bleeding coagulation is hastened by ( $\mathrm{I}$ ) temperature a little over that of the body; (2) contact with foreign matter; (3) injury to the vessel walls; (4) agitation; (5) addition of calcium salts; $(6)$ injection of nucleo-protein into the circulation, causing intra-vascular clotting.

Coagulation is hindered or prevented by $(\mathrm{I})$ a low temperature (in a vessel cooled by ice coagulation may be prevented for an hour or more); (2) the addition of a large quantity of neutral salts such as magnesium sulphate (known as Epsom salts), or, again, by sodium sulphate (known as Glauber's salts); (3) addition of a soluble oxalate, fluoride, or citrate; (4) injection of commercial peptone (which consists of proteoses) into the circulation of the living animal; (5) injection of leech extract into the blood-stream or contact from a leech bite; (6) contact with the living vascular walls of the bloodvessels; (7) contact with oil.

The cause of coagulation may be briefly stated as follows: When blood is circulating within the living walls of the bloodvessels, one of the constituents of the plasma, a protein of the globulin class called fibrinogen, exists in a soluble form. When the blood is shed the fibrinogen molecule is altered in such a way that it gives rise to the comparatively insoluble material, fibrin.

The question now arises, what causes the transformation of fibrinogen into fibrin? The answer to this question is that the change is due to the activity of an enzyme which is called fibrin ferment, or thrombin.

The importance of the nature of an enzyme is very great, as the chemical action of bacteria, whether harmful or otherwise to the human system, is due to the presence of an enzyme.

Enzyme is the term used by Kuhne for the soluble ferments. The word is also used for any ferment formed within the living organism or any chemical or hydrolytic ferment as distinguished from organized ferment such as yeast. Enzymes are most active between $86^{\circ}$ and $95^{\circ} \mathrm{Fah}$., and are destroyed by boiling. The word "fermentation" was first applied to the change of sugar into alcohol and carbonic acid by means of yeast. The evolution of carbonic acid causes bubbling and frothing, hence the term "fermentation." The agent, yeast, which produces this is called the ferment. The curdling of milk and the formation of vinegar from alcohol are brought about in a similar way. The complex changes known as putrefaction, which are produced by the 
various forms of bacteria, also come under the same category. That the change or fermentation is produced by these organisms is shown by the fact that it occurs only when the organisms are present and stops when they are removed or destroyed by a high temperature or by antiseptics.

Enzymes are also formed by the cells of the higher organisms, both in animal and vegetable life. Familiar instances of these are found in the saliva, which contains as its active constituent a substance called ptyalin. This is called the starch-splitting enzyme of saliva. There is also pepsin, the protein-splitting enzyme of the gastric juice. The substance upon which the enzyme acts is spoken of as the substrate. The essential facts concerning enzyme action can be illustrated in the following tabular way:

\begin{tabular}{|c|c|c|c|}
\hline The Living Cell. & $\begin{array}{c}\text { The } \\
\text { Enzyme } \\
\text { produced. }\end{array}$ & The Substrate. & $\begin{array}{c}\text { The Resulting Products of } \\
\text { Action. }\end{array}$ \\
\hline $\begin{array}{l}\text { The yeast cell } . . \\
\text { The salivary cell.. } \\
\text { The gastric cell .. }\end{array}$ & $\begin{array}{l}\text { Zymase } \\
\text { Ptyalin } \\
\text { Pepain }\end{array}$ & $\begin{array}{l}\text { Dextrose } \\
\text { Cooked starch } \\
\text { Protein }\end{array}$ & $\begin{array}{l}\text { Alcohol and carbon dioxide } \\
\text { Dextrin and maltose } \\
\text { Proteoses, peptones, and } \\
\text { amini acids }\end{array}$ \\
\hline
\end{tabular}

In the coagulation or clotting of the blood, therefore, the change is due to the activity of an enzyme called thrombin or fibrin ferment.

This enzyme or thrombin does not exist in healthy blood contained in healthy bloodvessels, but is formed by the disintegration of the blood platelets and colourless corpuscles, which occurs when the blood leaves the bloodvessel walls, or comes into contact with foreign matter. Hence the blood does not coagulate during life.

There are also present in the blood substances called antiferments which are active during life. Antithrombin neutralizes any tendency of the blood to clot in the vessels of a healthy person; in a similar manner the antiferments to the ferments of digestive fluids such as antipepsin and antitrypsin are efficacious in preventing the stomach and intestines from undergoing self-digestion.

Having described briefly the enzyme or fermenting action of thrombin, it is necessary to allude to what has been discovered 
regarding the origin of thrombin. Like other enzymes it is preceded by a mother-substance called zymogen. This zymogen is called thrombogen, and there appear to be two necessary agents concerned in the conversion of thrombogen into thrombin. One of these necessary agents is the action of calcium or lime salts, the other is the presence of an activating agent called thrombokinase. The part played by calcium salts is well illustrated by the fact that coagulation is prevented by the decalcification of the blood. The calcium salts are only necessary for the formation of thrombin or fibrin ferment, and not for the action of fibrin ferment or fibrinogen. Fibrin is thus not a compound of calcium and fibrinogen. The second activating agent, called thrombokinase, is not only liberated from the blood-corpuscles but it is obtained from many other tissues. The chemical action, therefore, of the coagulation of the blood takes place by the formation of three chemical substances produced from three distinct parts of the blood, which are:

I. The blood plasma-gives up fibrinogen.

2. The blood platelets
and leucocytes give up thrombogen.

3. The corpuscles and tissues

blood flows

The chemical action which takes place immediately upon the production of these three substances is that thrombokinase acts upon thrombogen to produce thrombin, which then unites with fibrinogen to produce fibrin, which completes the clot. The following tabular scheme shows the order of the coagulating action :

Three separate parts of the blood producing

the three foundation materials are:

The corpuscles and tissues, over which escaping blood flows,

give up

Thrombokinase activates thrombogen, which in the presence of calcium salts produces the active enzyme. and leucocytes give up
The blood platelets

Thrombin, which acts upon the fibrinogen, thus producing fibrin or clot.

The whole process when completed is called coagulation or thrombosis. 
- We have completed the preparation of the outline of the circulation of the blood and its vital properties as it affects the dental operator, but it must be understood by our readers that although the whole mechanism of the circulation appears so comprehensive, yet it by no means covers the subject as reviewed by the anatomist, the physiologist, and the pathologist. The details which are involved in our dental operating will be noted as we proceed. 


\section{CHAPTER VIII}

\section{THE NERVOUS SYSTEM FROM A DENTAL POINT OF VIEW}

THE nervous system is a term applied to the whole machinery of. the nerves taken collectively, and is the most complicated and the most highly organized of the various systems which make up the human body. It may be divided into two parts-viz.:

I. The central nervous system.

z. The peripheral nervous system.

The central nervous system consists of:

I. An upper expanded portion called the brain, which is contained within the cranium.

2. A lower, elongated, nearly cylindrical portion called the spinal cord, which is lodged in the vertebral canal.

These two portions are continuous with one another at the level of the base of the cranium.

The peripheral nervous system consists of a series of nerves, by which the central nervous system is connected with the various tissues and organs of the body. For descriptive purposes these nerves may be arranged in two groups:

Cerebro-spinal nerves.

Sympathetic nerves.

The arrangement, however, is an arbitrary one, since the two groups are ultimately connected and closely intermingled.

The cerebro-spinal nerves run in pairs, which number fortythree on either side. Twelve pairs of cranial nerves are attached to the brain, and thirty-one pairs of spinal nerves attached to the spinal cord. They are associated with the functions of the special and general senses, and with the voluntary movements of the body.

The sympathetic nerves transmit the impulses which regulate the movements of the viscera, determine the calibre of the blood- 
vessels, and control the phenomena of secretion. In relation with them are two rows of central ganglia, situated one on either side of the middle line in front of the vertebral column. These ganglia are intimately connected with the spinal cord and nerves, and are also joined to each other by vertical strands of nerve fibres so as to constitute a pair of knotted cords, called the gangliated cords of the sympathetic nerves, which reach from the base of the skull to the extreme end of the vertebral column.

The nervous system is built up of the nervous and non-nervous tissues. The latter is made up of neuroglia and bloodvessels together with certain enveloping membranes. Neuroglia is the reticulated framework or skeleton-work of the substance of the brain and spinal cord. It is similar to areolar tissue, which is widely diffused through the body, and composed of white and yellow fibres, the former imparting to it strength, and the latter elasticity. The two kinds of fibres interlace with each other again and again in the most complex manner. The interstices left between them are of very unequal size, and should not be called (as they were for a long time) cells. Neuroglia is composed of cells and fibres, the latter being prolonged from the cells. It is a connecting tissue that supports brain and nervous matter.

The nervous tissues of the body comprise the brain, the spinal cord, the cranial, spinal and sympathetic nerves, and the ganglia associated with them. The nervous tissues are found microscopically to be composed of nerve cells and their various processes, together with the supporting tissue called neuroglia, which, however, is only found in the brain and spinal cord.

Certain long processes of the nerve cells are of special importance, and it is convenient to consider them apart from the cells; they are known as nerve fibres.

The brain is composed of fibres or fasciculi, arranged in some parts longitudinally, in others interlaced at various angles by cross fibres, connected and held together by a delicate areolar web, which is the bond of support of the entire organ. It is enveloped by three lining membranes:

The dura mater.

The pia mater.

The arachnoid. 
The brain substance is of two kinds, differing in density and colour:

A grey or cortical substance, and

A white or medullary substance.

The grey substance forms a thin lamella over the entire surface of the convolutions of the cerebrum and of the laminæ of the cerebellum, but it is likewise found in the centre of the spinal cord.

The microscopic elements of the brain are white nerve fibres measuring from $\frac{1}{5000}$ to $\frac{1}{14000}$ of an inch in diameter, grey nerve fibres which are from one-half to one-third less than the white in diameter, and nerve cells measuring between $\frac{1}{300}$ and $\frac{1}{1250}$ of an inch in diameter, also nerve granules between $\frac{1}{5000}$ and $\frac{1}{10000}$ of an inch in diameter, with a varying number of pigment granules.

The weight of the human brain is from $2 \mathrm{lb} .5 \frac{1}{2} \mathrm{ozs}$. to $3 \mathrm{lb}$. I oz. The chemical constituents of the brain are albumen and fatty matter, including two acid compounds containing a large amount of phosphorus.

The functions of the brain are for the manifestation of the intellectual faculties - such as the emotions, passions, volitionand of sensation. The evolution of nerve force connected with mind emanates directly from the hemispherical ganglia.

The spinal cord, by its connection with the brain, is the essence of combined movements. The brain alone furnishes conditions necessary for intelligence, the spinal cord for movement; and together they connect the balancings and co-ordination of motor and sensific power.

The principal functions of the nerves are those of sensation and volition, motor and reflex action. The sensory actions are produced by the afferent or centripetal nerves, the motor by the efferent or centrifugal nerves.

Under the microscope nervous tissue is found to consist essentially of nerve cells and their branches. The nerve cells are contained in the brain and spinal cord, and in smaller collections of cells on the course of the nerves called ganglia. The part of the nerve centres containing cells is called grey matter. Long branches of the nerve cells are known as nerve fibres. These become sheathed, and are contained in the nerves and in the white matter of the brain and spinal cord. The bodies of nerve cells differ in size, shape, and arrangement. Nerve fibres are of two kinds-medullated and non-medullated. Medullated nerve 
fibres are found in the white matter of the nerve centres and in the nerves originating from the brain and spinal cord. Nonmedullated fibres occur in the sympathetic nerves.

A nerve is composed of a number of bundles of nerve fibres bound together by connective tissue. The nerve fibres which form the conducting portions of the nervous system may be classified into three main groups, according to the direction in which they normally conduct nerve impulses. These three classes are:

I. Efferent nerve fibres.

2. Afferent nerve fibres.

3. Intercentral nerve fibres.

Efferent or centrifugal nerves are those which conduct impulses from the central nervous system to other parts of the body. When, for instance, there is a wish to move a limb, the impulse starts in the brain and travels a certain distance down the spinal cord, it leaves the spinal cord by one or more of the spinal nerves and so reaches the muscles of the limb, which are then thrown into contraction. Such nerves are called motor nerves.

The efferent nerves are classed as follows:

(a) Motor.

(b) Accelerator.

(c) Inhibitory.

(d) Secretory.

(e) Trophic.

The motor nerves go to voluntary muscles and also involuntary muscles, such as the vasomotor nerves, which supply the muscular tissue in the walls of arteries.

Acceleratory nerves are those which produce an increase in the rate of rhythmical action. An instance of these is seen in the sympathetic nerves that supply the heart.

Inhibitory nerves are those which cause a slowing in the rate of rhythmical action, or, it may be, its complete cessation. Inhibitory nerves are found supplying many kinds of involuntary muscle. A very typical instance is found in the inhibitory fibres of the heart, which are contained within the trunk of the vagus nerve.

Secretory nerves are found supplying many secreting glands, such as the salivary glands, gastric glands, and sweat glands. The impulse which travels down a secretory nerve stimulates secretion in the gland it supplies. 
Trophic nerves are those which control the nutrition of the part they supply.

Afferent or centripetal nerves are those which conduct impulses in the reverse direction-namely, from all parts of the body to the central nervous system. When one feels pain in the finger, for instance, the nerves of the finger are stimulated, an impulse travels up the nerves to the spinal cord and then to the brain. The mental process set up in the brain is called a sensation. The sensation, however, is referred to the end of the nerve where the impulse started, and the sensation of pain does not appear to occur in the brain, but in the finger. This is an instance of a sensory nerve. The nerves of sensation may be grouped as follows:

I. The nerves of special sense, such as of sight, hearing, taste, smell, and touch.

2. The nerves of general sensibility-that is, of a vague kind of sensation not referable to any of the special senses, as, for instance, we may take the vague feelings of comfort or discomfort in the interior of the body.

3. The nerves of pain.

Intercentral nerves are those which connect nerve centres together; they connect different parts of the brain and of the spinal cord to one another, and are very complex in their arrangement.

The nervous system, from a dental point of view, is a subject much too vast for us even to lay down a brief description which is at all coherent or even intelligible, but as parts of the brain, spinal cord, and the nerves emanating therefrom are involved in some way or another in our daily work, it will be as well for us to review them in a general way here and refer to them again in future chapters as they arise.

The brain being the presiding organ of the body its functions are such that its delicate intricacies of mechanism must be always balanced, otherwise dislocation or partial dislocation of the system occurs. The patient's general health may be impaired without the dentist knowing it, or the imagination may produce nervous phenomena which we call shock, or more serious still maybe the condition of the brain whereby it is affected by drugs used as anæsthetics, both local and general. Again, the brain may be made to respond to certain drugs for the purpose of 
counteracting these conditions, which shows that the brain is in itself a most wonderful and complicated piece of mechanism, to which the most highly complicated electrical apparatus is as nothing when compared with it.

The brain consists of a large mass in front called the cerebrum, and a smaller mass behind called the cerebellum, which is placed at the base of the cerebrum, and a third division called the medulla oblongata. But this classification is not quite satisfactory because it is not sufficiently comprehensive of the many departments or sections of the brain.

In the early stages of embryology we find the brain being gradually formed from a tube called the neural tube. This tube is part of the spinal cord, and its anterior end becomes expanded into three vesicles which are called the primary cerebral vesicles. These are marked off from each other by intervening constrictions, and are classified as:

The fore-brain,

The mid-brain, and

The hind-brain.

The last one is continuous with the spinal cord. As the brain develops through being subjected to mental exercise, it finally becomes matured into the complicated structure that we find in the adult. Anatomists prefer to keep to this classification in studying the adult brain. The fore-brain develops into a large section called the cerebrum; the mid-brain does not develop into anything like the same size but joins the cerebrum to the hind-brain; the hind-brain consists of the cerebellum, the pons Varolii, and the medulla oblongata, or the bulb, as it is sometimes called. This latter is continued into the spinal cord. The above serves as a division of the main parts of the brain.

The cerebrum is divided along the middle into two parts, the right and left hemispheres, which remain united, however, by a thick transverse layer or band called the corpus callosum. This band consists of bundles of fibres which run from one hemisphere to the other. A good conception of the corpus callosum is obtained by examining a mesial section of the brain (or a good illustrated figure of this section), when it will be seen to form an arched structure about 4 inches in length. Its anterior extremity reaches to within about $I \frac{1}{2}$ inches of the frontal pole, and its posterior extremity about $2 \frac{1}{2}$ inches from the occipital pole of 
the hemisphere. The hemispheres are again imperfectly divided up into lobes by deep fissures. Shallower depressions called furrows or sulci subdivide the surface of the lobes into convolutions.

Under the corpus callosum are three ventricles or cavities which contain a watery fluid. The mid-brain, which joins the cerebrum or fore-brain to the cerebellum or hind-brain, is a short constricted portion which connects the cerebellum with the cerebral hemispheres. It is directed upwards and forwards and consists of:

I. A ventro-lateral portion composed of a pair of cylindrical bodies named the crura cerebri.

II. A dorsal portion consisting of four rounded bodies named the corpora quadrigemina eminences.

III. An intervening passage or tunnel called the aqueduct of Sylvius, which presents the original cavity of the midbrain and connects the third ventricle already mentioned with the fourth ventricle, which is situated beneath the third ventricle.

The hind-brain, consisting of the cerebellum, the pons Varolii, and the medulla oblongata, is situated behind the cerebrum, but in the basal portion of the skull. The cerebellum has long been a field of dispute among physiologists, and has never been satisfactorily cleared up or defined, but it is considered to bear an important part in the acts of balancing and the control of combinations of muscular movements as in walking. If the cerebellum of a bird be destroyed it is unable to keep its balance and falls over. Its principal function, however, has been supposed to be that of co-ordinating the action of the two sides of the body. Paralysis of the cerebellum produces a condition very like drunkenness: the two halves of the body do not work together, but act independently of each other, and the result is that the legs produce an unsteady gait, the eyes present a double image, and the tongue utters an indistinct speech.

The pons Varolii is a commissure or bridge which connects the hemispheres of the cerebellum. Just below the pons Varolii the medulla oblongata, or bulb, is situated. This section of the brain is a cranial prolongation of the spinal cord, of a similar structure, but differing by a peculiar arrangement of the strands of the cord before entering into and forming a connection with 
the brain. In it are found the great ganglionic centres which control respiration, circulation of the blood, deglutition or the act of swallowing, vomiting, etc. In the medulla there is a wonderful exchange centre whereby the nerve trunks, consisting of bundles

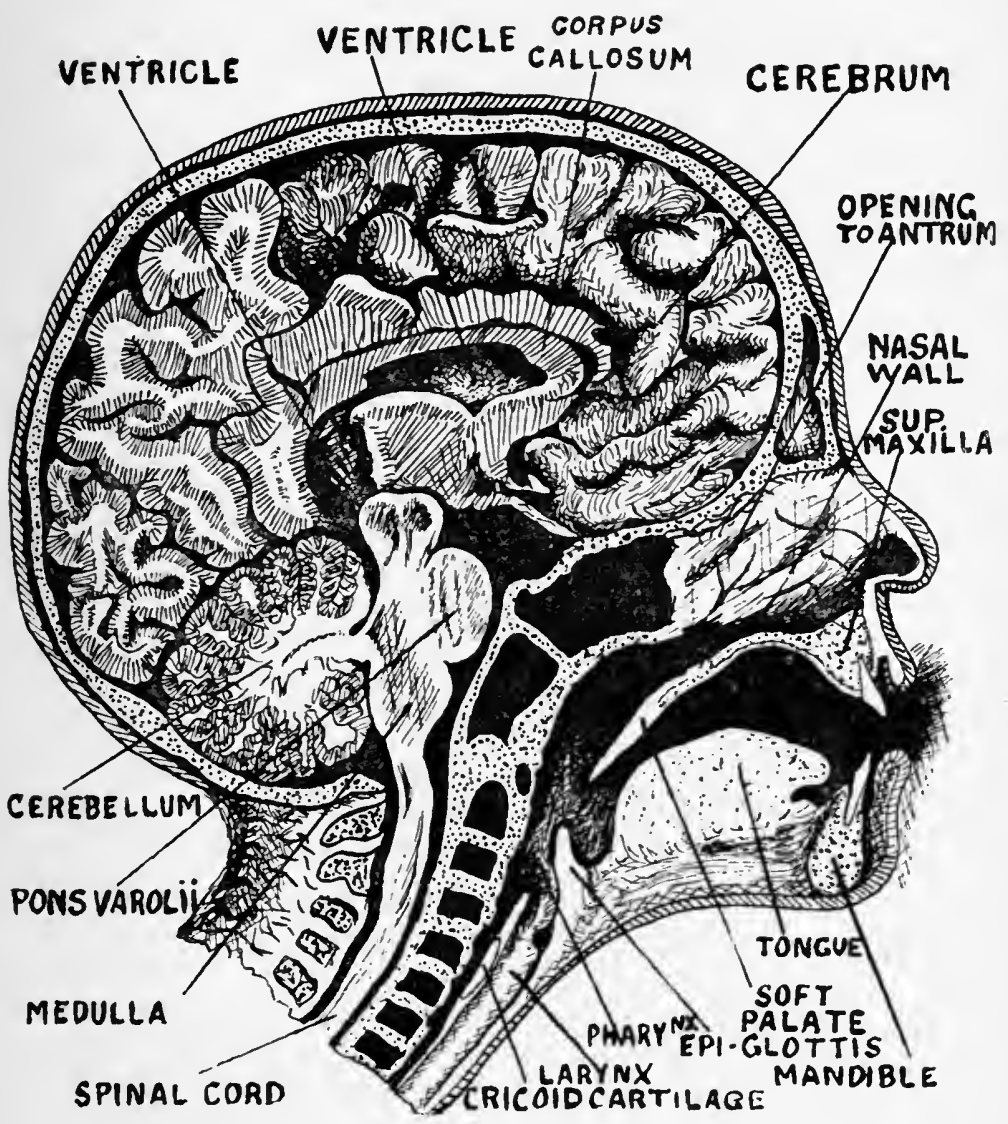

Fig. 30.-Sagittal Section of a Child's Head.

Prepared from a photograph to indicate the principal features involved in dental anatomy of the head.

of fibres from the spinal cord, cross each other on their way to their nervous centres in the brain itself. The crossing of the nerve fibres in the medulla or bulb on their way to different parts of the body results in the left side of the body being controlled by the right side of the brain, and vice versa (Fig. 30). 
Three membranes cover the brain, which comprise an outer tough one called the dura mater, a middle one called the arachnoid membrane, and an inner delicate one called the pia mater. This is an exceedingly sensitive and delicate membrane containing a very fine network of bloodvessels. The spaces between the membranes contain fluid, and this, with the fluid contained in the four ventricles, helps to protect the brain from harmful shock. The brain as a mass is composed of a layer of grey matter which covers the surface, dipping into the fissures and furrows, and also a mass of white matter consisting of nerve fibres, which lie underneath. On the quantity of grey matter and, in part, also, of the depth of the furrows, depends the intellectual power of the individual. Some of the fibres of the white matter carry messages from one part of the brain to another, others unite in bundles to form nerves, which pass out into the face and head and down into the spinal cord.

The spinal cord is a column of nerve substance connected above with the brain through the medium of the medulla, or bulb, situated in the spinal canal. In transverse section it is approximately circular, but the cord is not of the same size throughout its course. It is enlarged in two regions, one in the cervical or neck region and the other in the lumbar or loin region. These are the situations whence the large nerves for the supply of the limbs issue. The cord is composed of grey and white matter; the white matter is situated externally and constitutes its chief portion. The grey matter is in the interior, and is so arranged that in a transverse section of the cord it appears like two crescentic masses connected together by a narrower portion or isthmus called the posterior commissure or band. Passing through the centre of this isthmus in a longitudinal direction is a minute canal. In a transverse section this appears as a hole. This central canal is continued throughout its entire length, and opens above into the space at the back of the pons Varolii and medulla, called the fourth ventricle of the brain. The spinal cord, therefore, consists of two symmetrical halves separated by fissures, but joined in the middle by the commissures or bands with the canal containing fluid right in the centre. The white matter of the cord is made up of a bundle of medullated nerve fibres of different sizes running mainly in a longitudinal direction, and also of a supporting material which is of two kinds: firstly, an ordinary fibrous connective tissue with elastic fibres in which 
the bloodvessels are carried, and secondly, of neuroglia, which supports the nerve fibres. The grey matter of the cord consists of nerve fibres, most of which are very fine and delicate; also of nerve cells with branching processes, and of an extremely delicate network of fibrillæ of axis cylinders and other delicate structures. We will now proceed to point out the several parts of the nervous system in their functional aspects, which with some reflection will show the parts we can take more or less into consideration in operating on our patients.

There is first of all the patient's own control of himself. This function of the brain lies in a part called the cortex.

\section{The Cortex of Grey Matter.}

The cortex to which we refer is termed the cortex of the cerebrum or the cerebral cortex. This is generally supposed to be the seat of thought, consciousness, volition or will-power, and of all the higher attributes of the animal. These facts have been demonstrated mainly by:

I. Comparisons of the attributes of various animals and the development of their nerve centres.

2. Investigations of the attributes of animals congenitally defective in portions of the nervous system.

3. Observations of the losses inflicted by definite lesions of injury or disease upon the nerve centres.

4. Artificial infliction of cerebral inperfections such as the experimental removal of portions of the brain substance.

By these and other means the foregoing facts have been demonstrated to be highly probable.

A reflex act, however orderly, must immediately follow the stimulus that causes it. A physical act, on the contrary, may be the result of stimulus received long before. The storage of this stimulus is called memory. In all probability no stimulus is ever lost, nothing is really ever forgotten, and the most trifling incidents of the distant past are often revived in dreams, while the repetition at another time and place of some associated stimulus, such as a peculiar scent or sound, will often reproduce faces, words, or scenes, and even experiences of long ago that we had fancied had faded altogether from our minds.

The power to exercise conscious thought, occurring either through the immediate stimulus of any present experience of 
pleasure or pain, or through being called up by a store of past experiences and the exercise of will-power to do something mentally, or some definite action physically, is contained in the cerebral cortex.

The cerebrum consists of two halves called the cerebral hemispheres, which are separated by a deep longitudinal fissure, and connected by a large band of transverse commissural fibres known as the corpus callosum. The interior of each hemisphere contains a cavity of complicated shape called the lateral ventricle. The lateral ventricle of each hemisphere, being the first and second ventricles, opens into the third ventricle. Each hemisphere is covered with grey matter, which passes down into the fissures. This surface grey matter is called the cerebral cortex. The amount of this outside crust of grey matter varies directly with the amount of convolution of the surface.

\section{Lobes and Convolutions of the Cortex.}

The surface of the brain is marked by a great number of depressions which are called fissures or sulci, and it is this folding of the surface that enables a very large amount of the precious material called the grey matter of the cortex to be packed within the narrow compass of the cranium. In the lowest vertebrates the surface of the brain is smooth, but going higher in the animal scale the fissures make their appearance, reaching their greatest degree of complexity in the higher apes and in man. In an early embryonic stage of the human fotus the brain is also smooth, but as development progresses the sulci or fissures appear, until the climax is reached in the brain of the adult. The sulci, which make their appearance first, both in the animal scale and in the development of the human fœtus, are the same. They remain in the adult as the deepest and best-marked sulci; they are called the primary fissures or sulci, and they divide the brain into lobes. The remaining sulci, called the secondary fissures or sulci, further subdivide each lobe into convolutions. A first glance at an adult human brain reveals what appears to be a hopeless puzzle. This, however, is reduced to order when one studies the brain in different stages of development or compares the brain of man with that of the lower animals.

There are two principles involved in the working of the nervous system as a whole, which we must examine in order to under- 
stand how this vast and complicated mechanism carries out its functional activity. These principles are-

Nerve force.

Reflex action.

Nerve force has reference to the nature of the message to and from nerve cells by means of nerve fibres. We are accustomed to speak of this particular form of energy under the name of nerve force. This may be defined as the particular kind of

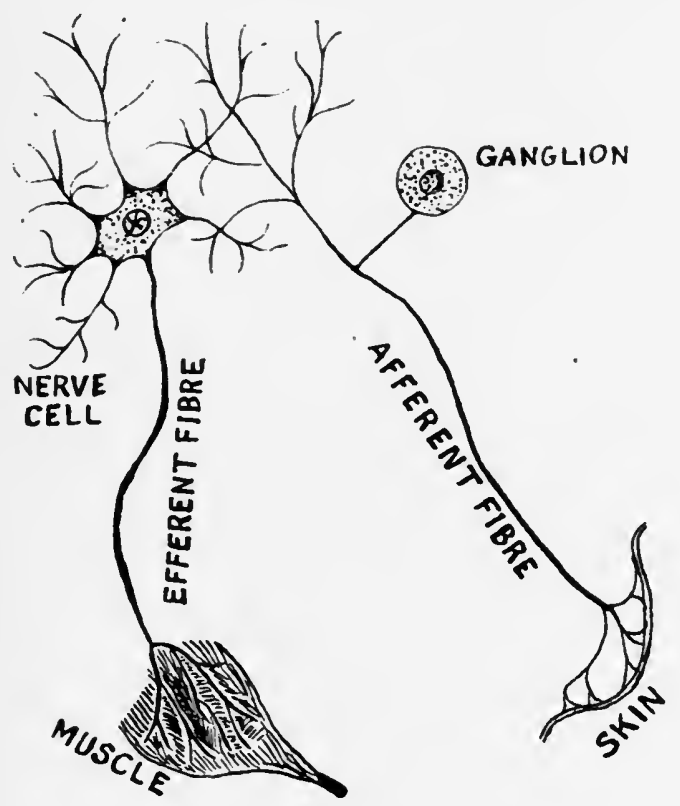

Fig. 31.-Diagram to illustrate Nervous Reflex Action.

energy which is exercised and originated by nerve cells, and by them alone. It may be regarded, therefore, as a property of the protoplasm or living matter of which nerve cells are composed. The rate at which nerve force travels has been calculated by means of delicate instr uments (see diagram, Fig. 3I).

Reflex action is the principle on which the nervous business of the body, so to speak, is carried on. Actions result from the transmutation of afferent into efferent impulses, by means of the irritable protoplasm of a nerve cell. They constitute the function of the spinal cord, the grey matter of which consists of 
a multitude of reflex centres. There is also another set of ganglia, which are related anatomically in a similar way to some of the cranial nerves, and physiologically with the involuntary muscles and glands in the head region, as well as with some of the thoracic and abdominal organs. Thus we have the ciliary ganglion in connection with the third cranial nerve, also the spheno-palatine, otic, and submaxillary, in connection with other cranial nerves.

In order that reflex action may be carried out, we find the constitution of the nervous system presenting us with a definite scheme whereby all the wants of the body in respect of instantaneous results can be brought about. This principle will account for the unconscious movements which may be observed during our operations on the teeth, when the pupil of the eye may suddenly dilate, or movements of various parts of the body may occur. This principle is again demonstrated in a very important manner when a patient is under the influence of a general anæsthetic.

For a reflex action to take place three things are necessary: (I) An afferent or sensory nerve, which carries the impulse from a sensory organ such as the skin, or the fibres of the dental pulp, or any other sensory part of the body; (2) a nerve centre, consisting of nerve cells, to receive the afferent or sensory impulse and send out the efferent impulse to the muscle involved; and (3) an efferent or motor nerve along which the efferent impulse may travel. 


\section{CHAPTER IX}

\section{THE PARTS OF THE NERVOUS SYSTEM WHICH ARE DIRECTLY INVOLVED IN DENTAL OPERATING}

THE third division of the brain, called the medulla oblongata, or bulb, is the continuation upwards of the spinal cord. It is not more than $I$ inch in length and it may be regarded as commencing at about the level of the magnum foramen at the base of the skull in the occipital bone. From this it proceeds upwards in a very nearly vertical direction and ends at the lower border of the pons Varolii. At first its girth is similar to that of the spinal cord, but it rapidly expands as it approaches the pons, and consequently it presents a more or less conical form.

The medulla oblongata, although unconnected with any of the higher operations of the mind, is an extremely important part of the brain through its position in the nervous system. Through it run the nerve fibres passing from the brain to the body, whilst its own nerve cells or centres give origin to certain important nerves governing the heart, breathing, swallowing, and the vasomotor system (see Fig. 30).

Another feature of the medulla is that in its lower part the nerve fibres passing from the brain on each side cross to the opposite side of the spinal cord. This is called the " decussation of the pyramids." This feature is of great interest, as it shows the reason why an injury on one side of the brain is seen to affect the other side of the body. A person stricken with apoplexy on the right side will be found to have an injury or lesion on the left side of the brain, or vice versa.

Lying above the medulla we find a bandlike structure known as the pons Varolii. This consists of nerve fibres which connect the cerebrum with the cerebellum and the medulla. Above it are two white masses which pass upwards to the under surface of the cerebrum. These masses are known as the crura cerebri. They carry the nerve fibres from the medulla into the cerebrum itself. The pons Varolii may be regarded, as its name indicates, as a 
bridge connecting the medulla, not merely with the cerebrum, but with the cerebellum also.

The nerves that are directly involved in the dental tissues are the fifth pair of cranial nerves. The trigeminal, trifacial or fifth nerve arises from the surface of the pons Varolii on its outer part by two roots, a large sensory root and a small motor root. The two roots proceed forward in the posterior fossa of the base of the skull and pierce the dura mater near the superior border of the petrous portion of the temporal bone. The large sensory root gradually conceals the small motor root in its course forwards and expands into a large flattened ganglion called the Gasserian ganglion, which occupies a cavity in the dura mater which is situated on a depression near the apex of the petrous bone. It is somewhat crescentic in shape, with its convexity directed forwards. Internally it is in relation with the internal carotid artery and the posterior part of the cavernous sinus. The motor root runs forward and passes below the ganglion without having any connection with it whatever at the present. The Gasserian ganglion gives rise to three great divisions of the fifth nerve. These are branches which are termed:

I. The ophthalmic nerve or division.

2. The superior maxillary nerve or division.

3. The inferior maxillary nerve or division.

The ophthalmic nerve passes forward to the orbit through the sphenoidal fissure. It supplies the upper region of the nose, around the orbits, and the frontal region (see Fig. 25).

The superior maxillary nerve courses forward from the Gasserian ganglion in relation to the lower part of the cavernous sinus. Passing through the foramen rotundum it traverses the spheno-maxillary fossa (see Fig. 25). It enters the orbit as the infra-orbital branch through the spheno-maxillary fissure, and passing through the infra-orbital canal it finally appears on the face through the infra-orbital foramen. There are a great many branches of each division of the fifth nerve as a whole, and its ramifications do not all enter into our studies, therefore we mention those only which come within our scope (see Fig. 32).

In the spheno-maxillary fossa the superior maxillary nerve gives off (I) two short, thick, spheno-palatine nerves, which are the short or sensory roots of the palatine ganglion, called Meckel's ganglion; (2) a posterior dental nerve which descends 
through the pterygo-maxillary fissure to the outside of the jaw and proceeds forwards through small foramina in the alveolar wall and supplies the molar teeth. This nerve forms a fine plexus with the middle dental nerve before reaching the fine filaments that enter the roots of the teeth. The infra-orbital nerve is the terminal branch of the superior maxillary nerve, which enters the orbit through the spheno-maxillary fissure and traverses the infra-orbital canal, which finally comes out on the face just below the orbit. In the infra-orbital canal it gives off a middle dental branch, which descends to the biscuspid teeth, and also an anterior dental nerve. These last two branches descend the bony canals inside the outer wall of the antrum and reach the alveolar arch, where they supply the two bicuspid teeth, and the anterior nerve supplies the incisors and canines.

The spheno-palatine, or Meckel's ganglion, occupies the upper part of the spheno-maxillary fossa. It is a small reddish-grey ganglion, suspended from the superior maxillary nerve by the two short spheno-palatine branches which constitute its sensory roots. The branches from this ganglion are: (r) The pterygopalatine or pharyngeal branch, which passes backwards through the pterygo-palatine canal to supply the mucous membrane of the roof of the pharynx; (2) the posterior palatine nerves, three in number, are directed downwards to the palate through the posterior palatine canals. The large posterior emerges on the under surface of the palate through the large posterior palatine canal and at once separates into numerous branches to supply the mucous membrane of the hard and soft palate. The small posterior palatine nerve descends through the small posterior palatine canal, and piercing the tuberosity of the palate bone, is distributed to the mucous membrane of the soft palate, uvula, and tonsil. The accessory posterior palatine nerves are one or more small twigs, which pass through accessory posterior palatine canals and supply branches to the mucous membrane of the tonsil, soft palate, and uvula. There are also other branches to the nose. The naso-palatine nerve, after passing through the spheno-palatine foramen, crosses the roof of the nose, and extends obliquely downwards and forwards along the nasal septum, grooving the vomer bone in its course to reach the anterior palatine canal through the foramina of scarpa. The nerves of this branch from each side of the maxillæ unite within 


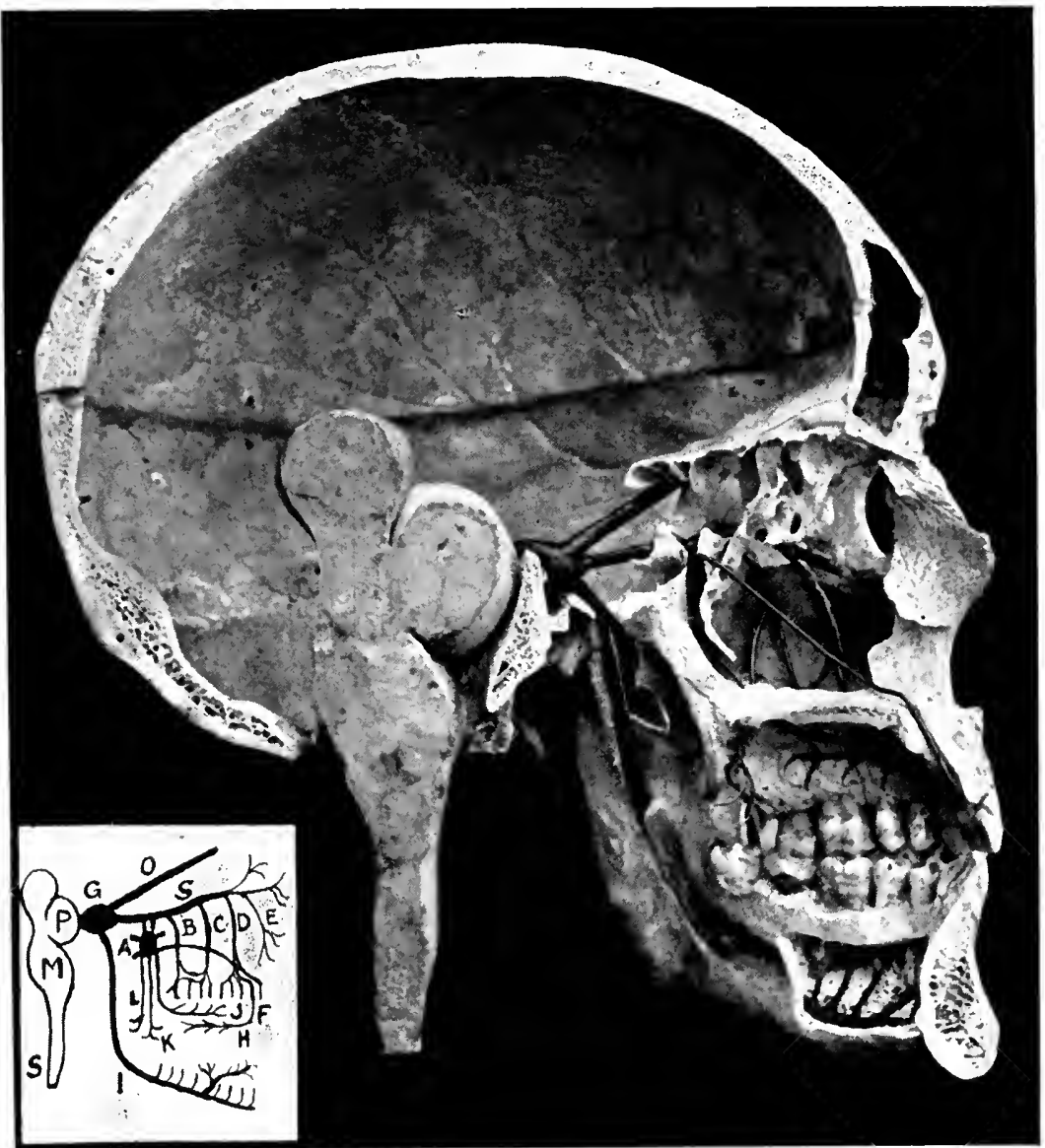

Fig. 32.-Demonstration of the Fifth Cranial Nerve,

Showing the Position of its Origin, the Dental Branches to the Roots of the Teeth and also to the Palate.

Its origin consists of a large sensory trunk and a small motor trunk arising on the surface of $P$, the PoNs VARoliI. The fibres of the sensory root pass downwards through $\mathrm{NI}$, the Medulla into $\mathrm{S}$, the SpINAL CORD as far as the second cervical nerve. The fibres of the motor root pass upwards and backwards to the walls of the Aqueduct of Sylvius. The sensory trunk proceeds a short way and expands into $\mathrm{G}$, the Gasserian Ganglon. It then separates into three divisionsO, the Ophthalmic Branch, S, the Superior Maxillary Branch, and I, the Inferior Maxillary Branch. 
The Superior Maxillary Branch passes through the Foramex Rotundum, branching into A, MECKEL's GANGLIon, which sends off L, Accessory Posterior Palatine, K, Small Posterior Palatine, J, Large Posterior Palatine, and F, Naso-Palatine, passing through the Anterior Palatine Canal. It runs then across the Spheno-Maxillary fossa and enters the Orbital canal, which is situated immediately below the Orbital surface of the Superior Maxilla (see Fig. 28), where it becomes the Infra-Orbital nerve, passing through the Infra-Orbital Foramen to reach the face, E. Just before entering the Orbital, it sends off B, Posterior Dental, through the posterior wall of the Antrum to the molar roots; in the canal, C, Middle Dental to bicuspid roots, forming a loop with Posterior Dental; D, Anterior Dental, through the solid portion of Superior Maxilla to the roots of canine and incisors.

I, The Inferior Maxillary Branch runs downwards through the Foramen Ovale. It is formed by the union of the sensory trunk with the small motor trunk and supplies fibres to a large number of muscles. After giving off several branches it enters the Inferior Dental foramen as the Inferior Dental Nerve, which then passes down to supply the molar roots and the second bicuspid, where it sends off the Mental Branch through the Mental Foramen. The Inferior Dental continues from this point as the Incisive Branch supplying fibres to the canine and incisors.

The specimen shows the relative positions of the SPINAL CORD, the MEDUlla, the Pons VaroliI, the Crus Cerebri (behind and above the Pons); on its posterior border lies the AQueduct of Sylvius, which connects the third ventricle with the fourth ventricle of the Brain. The nasal wall and part of the Sphenoid Bone has been well cut away to show the positions of the nerve branches. The Infra-orbital Canal can be seen running along the floor of the orbit, but the Infraorbital Foramen is hidden by the remains of the nasal wall. The depth of the Antrum is shown, but the floor which descends to the apices of the molar and bicuspid teeth is hidden by the palate portion of the Superior Maxilla. The Spheno-Maxillary fossa can be seen lying behind the posterior antral wall, where MEckeL's GANGLIoN may also be seen. The Posterior Palatine canals lie in the solid portion below. The Anterior Palatine canal is clearly shown and also the solid portion of the Superior Maxilla.

The cortical plate and part of the process has been removed from the internal side of the Mandible to show the fibres supplying the roots of the Iower teeth. The Inferior Dental Foramen is clearly seen. The Mental branch of the Inferior Dental Nerve may be seen passing upwards and outwards below the first bicuspid tooth of the Mental Foramen on the external surface of the jaw (see Fig. 34). The position of the Mental Foramen varies and is never constant; sometimes it lies between the Ist Molar and 2nd Bicuspid; in this specimen it is present immediately below the rst Bicuspid. Usually it is found between the roots of the ist and 2 nd Bicuspids. The section of the Mandible has been cut through at the centre of the prominence of the chin called the SyMPHYSIs. 
the foramen and, appearing on the palate, then branch out again, supplying the mucous membrane of the hard palate. They communicate posteriorly with terminal filaments of the large posterior palatine nerves.

\section{Third Branch of the Fifth Nerve.}

The inferior maxillary nerve distributes branches to the teeth and gums of the lower jaw, parts of the face and lower lip, and the muscles of mastication. It also supplies a large branch to the tongue. It is the largest of the three divisions of the fifth nerve, and is made up of two roots: a large sensory root proceeding from the inferior angle of the Gasserian ganglion, and a small motor root which passes beneath the ganglion and unites with the sensory root just after its exit from the skull at the foramen ovale (see Fig. 25). Immediately beneath the base of the skull the nerve divides into two trunks, the anterior and posterior. Previous to its division it gives off a recurrent branch and also a nerve to the internal pterygoid muscle (see Fig. 32).

The inferior dental nerve is the largest of the three branches of the inferior maxillary nerves. It passes downwards with the inferior dental artery, at first beneath the external pterygoid muscle and on the inside of the ramus of the lower jaw, and enters the dental foramen of the inferior dental canal, which is situated at a point approximately about an inch behind and a little above the level of the lower wisdom tooth; it then invariably passes quite close to the posterior root of the wisdom tooth, and then passes about a quarter of an inch below the molars and bicuspids. At a point which varies either between the bicuspid roots, but below them, or between the first molar and second bicuspid, there is an opening or foramen which allows a branch to pass out into the lower lips and cheek. This foramen is called the mental foramen. The nerve continues in the canal under the incisor teeth, and is called the incisive branch, and is continued onwards within the bone to the middle line and supplies the canine and incisor teeth.

It will be seen from the above description that the terminal branches of the three divisions of the fifth nerve emerge from foramina or openings on to the face. The terminal branch of the first division emerges through the supra-orbital foramen just above the top of the orbit. The second emerges through the infra-orbital foramen just below the floor of the orbit. The third 
passes through the mental foramen on the side of the lower part of the face in the region of the bicuspids. It will have well served our purpose if the varied intricacies of the branches of the fifth pair of nerves have been outlined clearly enough to account for the supply of all the roots of the teeth, the gums, and palate.

The nerves controlling the vasomotor system originate in the medulla of the brain. Each bloodvessel is thereby maintained in a state of moderate contraction, being neither unduly contracted nor unduly expanded. If some stimulus or other affects these nerve centres then the bloodvessels may either contract or dilate, according to the nature of the cause underlying the nervous impulses. In the first case, the contraction limits the bloodsupply by keeping the blood back in the arterioles, thereby causing pallor, or a condition of bloodlessness. On the other hand, if the nervous control causes the arterioles to expand or dilate a greater blood-supply is given out and produces a flushing or blushing condition, and deepens in redness accordingly. The heart's action is controlled by a special nervous mechanism set apart for the purpose of governing its movements. In ordinary health the heart beats normally through the controlling action of two sets of cardiac nerves. Embodied in the substance of the heart we find a mass of nerve cells known as cardiac ganglia. The greater number of these are engaged in stimulating or quickening the heart's action, but a smaller number are engaged in inhibiting or slowing the heart's action.

The inhibitory or slowing action is caused by the cardiac branches of the tenth pair of cranial nerves, called also the pneumogastric nerve, and the vagus or wandering nerve. The tenth nerve has its origin in the medulla. Its main course is from the neck to the abdomen, and it gives off large branches also to the respiratory passages, the pharynx, stomach, liver, and spleen.

The quickening or acceleratory action of the heart is caused by the cardiac branches of the sympathetic nervous system.

The cardiac branches of the vagus and the sympathetic nerves consist of efferent fibres. There are, in addition to these, some afferent or sensory nerves belonging to the heart. The main one is called the depressor nerve. This nerve passes from the heart and joins the vagus, then proceeding upwards to the medulla. This is regarded as the relief nerve of the heart. It is brought into action when, for some reason or other, the heart's action is 
much impeded, and when the heart has a tendency to become exhausted with increased work which it finds difficult to perform. In such a case an impulse from the heart is transmitted along the depressor nerve to the medulla, where it is received, and an impulse is then transmitted to the vasomotor centre, which causes the arterioles to dilate, especially those in the splanchnic area of the abdomen. In this way the heart is relieved from the resistance of the blood-pressure against which it becomes embarrassed.

The blood-supply to the teeth and immediate tissues is from the branches of the internal maxillary artery. The blood courses from the heart into the aorta, then through the common carotid artery to the external carotid artery, and then to the internal maxillary artery.

The various branches then take their name from the branches of the fifth pair of nerves.

The veins return the blood through the posterior and inferior dental canals, which follow closely the corresponding arteries. These veins, with others, form the pterygoid plexus. At the back of the plexus the venous blood empties into the internal maxillary vein, through the temporo-maxillary vein, then through the external jugular vein, through the innominate vein into the superior vena cava, emptying into the right side of the heart. 


\section{CHAPTER X}

\section{THE MUSCLES AND ORGANS IN THE IMMEDIATE VICINITY OF THE ORAL CAVITY}

\section{What are the Functions of the Oral Cavity?}

IN order to complete our outlines of the anatomical and physiological parts with which every dental operator's work is involved in carrying out one operation or another, the remaining parts for our consideration might very usefully be surveyed by raising the question, What are the uses or the functions of the mouth and oral cavity? In proceeding to endeavour to answer this question we would remark that the question is apparently a simple one, but the answer is a comprehensive one. In the first place it is somewhat difficult to arbitrarily define what is the extent or limitation of the oral cavity when applying the word "function" to it. The superficial surface of the cavity, which is covered by the mucous membrane, can be seen by the naked eye, and is thus easily estimated as to its size, but the consideration of the practical work which the oral cavity is constantly carrying out, involves the existence of deep structures, which are of vital importance to the operator's general work.

\section{These Normal Functions must not be interfered with by the Dental Operator.}

The functions of the oral cavity involve four separate processes:

I. The action of speaking.

2. The action of masticating.

3. The action of swallowing.

4. The action of expression.

These four distinct actions, which form the functions of the mouth, must necessarily be regarded as being the laws to which all dental operating must be subservient. If we construct a set of artificial teeth in an edentulous mouth we are at once involved in all four actions of the oral cavity, and although we may not 
always be able to harmonize these functions equally in inserting a set of teeth, yet they must receive our profound consideration, otherwise great discomfort arises to the wearer. After the natural teeth have been extracted, the bony jaws absorb, and the absorption goes on very often in the bone, quite a distance away from the actual area of the oral cavity. Also the bone will become absorbed on its alveolar surface to such an extent that it is difficult to obtain a correct or satisfactory alignment of the plate or denture.

The muscles are attached firmly by tendons, or closely bound cords of muscle fibres, to their proper place on the bones. In the upper and lower jaws we have to consider that there are numerous and powerful muscles attached to every part of them, and the existence of great shrinkage or contraction of the bone brings the fitting of the denture into a state of interference with the working of these muscles, which results in the denture being pressed by them, and pain ensues, or the plate is constantly being dislodged or rocked, leading to chafing and inflammation of some part, or a generally painful condition.

Again, the muscular attachments of the tongue, if interfered with, embarrass that organ and the action of speech is interfered with, or the act of swallowing may be made difficult. The various voluntary and involuntary facial expressions produced by the patient through the action of the facial muscles will often interfere with the setting of a denture.

We have also to consider the main purpose of fitting a denture, which is to restore the mechanism of mastication. If the elevation of the bite or the proper relation of the plane of the lower surface to the plane of the upper surface is not approximately correct, the muscles cannot work harmoniously, and the whole process is incomplete.

In filling teeth we have to consider also the nature of the fluids of the mouth, because they may be a source of infection to the teeth, thereby causing caries. Also the position of the salivary glands and their ducts will enable us to control the flow of saliva, so that the filling of cavities and the setting of crowns and bridgework may be properly carried out.

The act of deglutition, or swallowing, is often seriously affected when we administer either a local or a general anæsthetic, and a general conception of how this process is carried out will enable us to cope with any obstruction that interferes with it. 
The muscles of the mouth within and without and the salivary glands constitute, therefore, our subject in this chapter, and our description will extend from the lips and cheeks externally to the pharynx and larynx internally.

\section{Mastication and Expression.}

The primary functions of the oral cavity are the mechanical processes of mastication and swallowing or deglutition. The act of mastication is performed by the biting and grinding movements of the lower range of teeth against the upper ones, which is of a rotary nature. The lower jaw does not move up and down like a hinge, but travels within an elliptical circuit. Although the teeth in this way grind the harder parts of food, yet maceration is materially assisted by the simultaneous movements of the tongue and cheeks, which crush the softer portions against the hard palate and gums, and partly by returning the morsels of food again and again, as they are squeezed out from between them until they have been sufficiently chewed. With the process of mastication the food is incorporated with the saliva, and the more thoroughly this takes place the more ccmplete is the bolus, or mouthful of food, ready for its entrance into the stomach.

The functions of expression and mastication being carried out by means of various muscles, all of which participate in the action of mastication, the study of them is better carried out by classifying them into those sections:

I. The maxillary region.

2. The mandibular region.

3. The intermaxillary region.

4. The temporo-mandibular region.

5. Pterygo-mandibular region.

We do not propose to follow this classification, although it is a perfect one for the thorough study of all the muscles involved in those parts.

If we examine a face from the front we shall note that there are the lips and the orifice of the mouth. All the muscles run in pairs, so that we shall generally refer to one side of the face only, although it must be understood that there is a right and a left side to the face with the corresponding right and left muscles of the same name, unless otherwise stated. 


\section{The Muscles of the Front Part of the Mouth in the Upper Region.}

The orifice of the mouth consists of a muscular ring which is capable of contracting into a circle or extending into an oval form. This muscular ring is called the orbicularis oris, and consists of muscular fibres having different directions and surrounding the orifice of the mouth. These fibres are partially derived from the other facial muscles, which are inserted into the lips, and are partly fibres proper to the lips themselves. The orbicularis oris in its ordinary action produces the direct closure of the lips. By its deep fibres, assisted by oblique ones, it closely applies the lips to the alveolar arch. The superficial portion brings the lips together and also protrudes them forwards.

The muscular ring which we have described is further controlled by a series of muscles which work the area outside the lips. In the upper part of the face they extend from the orbit, and in the lower part of the face from the chin.

In the maxillary region, which includes the lips of the upper part of the mouth and just round the side near the bicuspids, we find these muscles:

Levator labii superioris proprius.

Levator anguli oris.

Zygomaticus major.

Zygomaticus minor.

The levator labii superioris arises from the lower margin of the orbit immediately above the infra-orbital foramen, some of its fibres being attached to the maxilla, others to the malar bone. These fibres converge and become inserted into the muscular substance of the upper lip. This muscle is the proper elevator of the upper lip, carrying it at the same time a little forwards. It assists in forming the naso-labial ridge, which passes from the side of the nose to the upper lip, and gives to the face an expression of sadness.

The levator anguli oris is attached to the maxilla just below the infra-orbital foramen and is inserted into the angle of the mouth just at the corners, and becomes intermingled with the zygomaticus major, the depressor anguli oris, and the orbicularis oris. This muscle raises the angle of the mouth at the sides.

The zygomaticus major is a slender muscle which arises from the malar bone, in front of the zygomatic suture, and descends 
obliquely downwards and is inserted into the angle of the mouth in a similar way to the levator anguli oris. This muscle draws the angle of the mouth backwards and upwards as in laughing.

The zygomaticus minor arises from the malar bone immediately behind the maxillary suture and passes downwards and becomes inserted in a similar way to the preceding ones. Its position is between the levator labii superioris and the zygomaticus major. This muscle being inserted into the outer part of the upper lip and not into the angle of the mouth, draws it'backwards, upwards, and outwards, and thus gives to the face an expression of sadness. This group of muscles is supplied by the facial nerve, which is the seventh pair of nerves. It consists of motor and sensory fibres.

\section{The Muscles of the Mouth in the Lower Region.}

The mandibular region, which takes in the lower lip and corners of the mouth, includes these muscles:

The levator menti.

Depressor labii inferioris.

Depressor anguli oris.

The levator menti is a small muscle situated on each side of the frænum of the lower lip; its fibres are attached on each side of the chin part of the jaw, called the symphysis, and pass upwards in the region of the incisors to the lower lip. This muscle raises and protrudes the lower lip, and at the same time wrinkles the integument of the chin, expressing doubt or disdain.

The depressor labii inferioris is a small quadrilateral muscle. It arises from the external oblique line of the mandible between the symphysis of the chin and the mental foramen and passes obliquely upwards and inwards, to be inserted into the lower lip, its fibres blending with the orbicularis oris. This muscle draws the lower lip down and a little outwards as in the expression of irony. The depressor anguli oris is triangular in shape, arising by its broad base from the external oblique line of the mandible, whence its fibres pass upwards to be inserted into the angle of the mouth, and is the antagonist of the levator anguli oris and the zygomaticus major; acting with the levator anguli oris it will draw the angle of the mouth directly inwards (Fig. 33).

From the corner of the mouth and running horizontally backwards lies the risorius muscle, which consists of a narrow bundle 
of fibres, and which arises in the covering of the masseter muscle and passes horizontally forwards and is inserted into the skin at the angle of the mouth against the orbicularis oris. This muscle

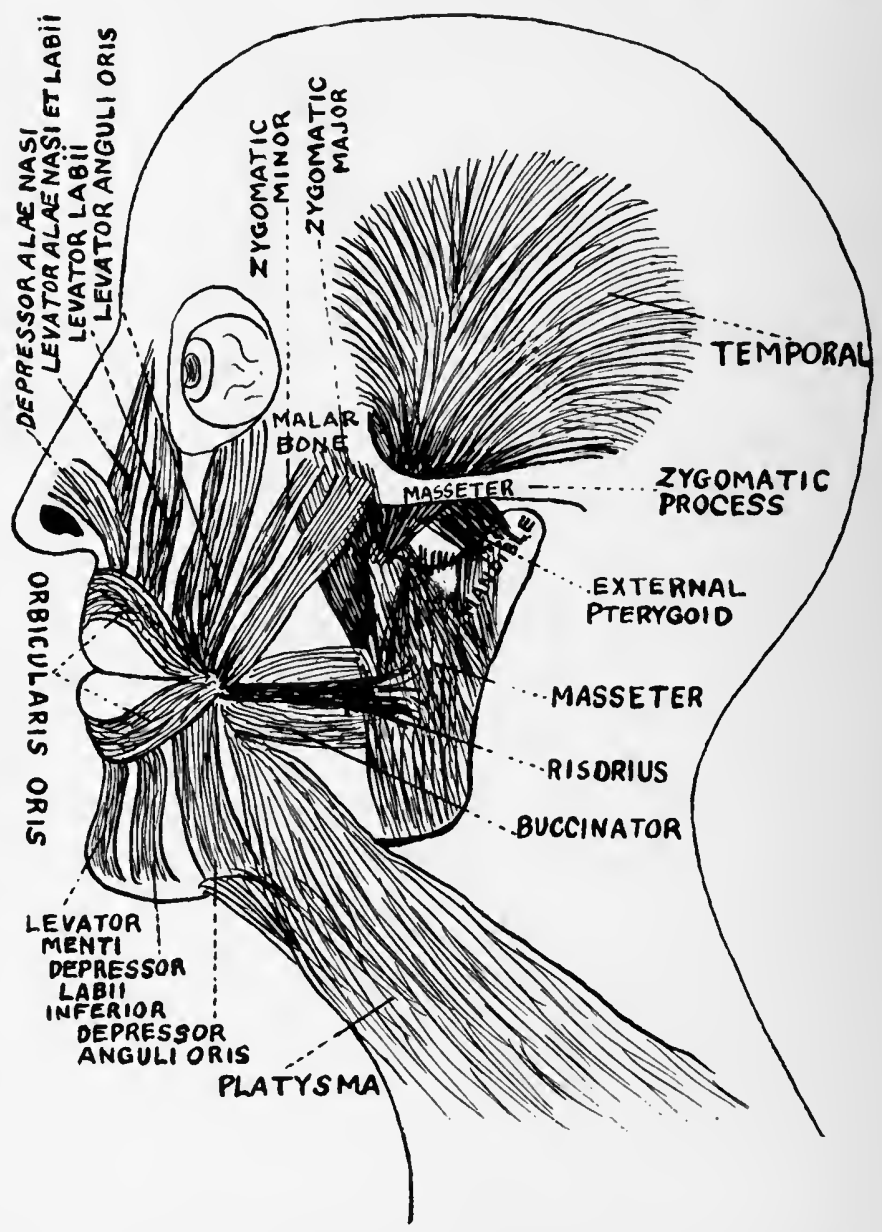

Fig. 33.-Scheme to indicate the Muscles of Expression and Mastication.

The external pterygoid can only just be seen. The internal pterygoid cannot be shown as it lies inside the angle of the mandible.

varies much in size and form. The risorius retracts the angles of the mouth and produces the unpleasant expression which is sometimes seen in tetanus and is known as " risus sardonicus." 


\section{The Muscles of the Side Region more directly involved in Mastication.}

Passing from the lips, where the muscles are largely involved in facial expression, as in speech, we come to the side of the jaws. The muscles here are powerful in their movement and action, and are employed to work the movements of the cheeks and the lower jaw.

The buccinator muscle is a broad thin muscle, quadrilateral in form, occupying the interval between the jaws at the side of the face. It arises from the outer surfaces of the alveolar processes of the upper and lower jaws corresponding to the three molar teeth. The fibres converge towards the angle of the mouth, where the central fibres intersect each other, those from below being continuous with the upper segment of the orbicularis oris, and those from above with the lower segment. The buccinator is in relation by its superficial surface, behind, with a large mass of fat which separates it from the ramus of the lower jaw, also from the masseter muscle and from a small portion of the temporal muscle. In front, the superficial surface of the buccinator is in relation with the zygomatic, risorius, levator anguli oris, and depressor anguli oris muscles. The deep surface is in relation with the buccal glands and mucous membrane of the mouth. The buccinators contract and compress the cheeks, so that during the process of mastication the foot is kept under the immediate pressure of the teeth. When the cheeks have been previously distended with air the buccinator muscles expel it from between the lips, as in blowing a trumpet. Hence the name, buccina, a trumpet.

The masseter muscle is a short, thick muscle, somewhat quadrilateral in form, consisting of two portions, a superficial and a deep one. The superficial portion, which is the larger, arises from the malar process of the maxilla, and from the anterior two-thirds of the lower border of the zygomatic arch. Its fibres pass downwards and backwards, to be inserted into the angle and lower half of the ramus of the mandib'e.

The deep portion of the masseter muscle is much smaller, and more muscular in texture. It arises from the posterior third of the lower border and the whole of the inside surface of the zygomatic arch. Its fibres pass downwards and forwards, to be inserted into the upper half of the ramus and the outer surface 


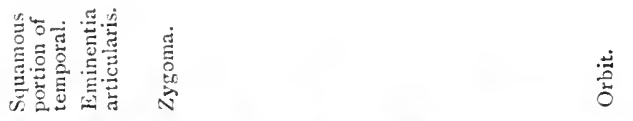

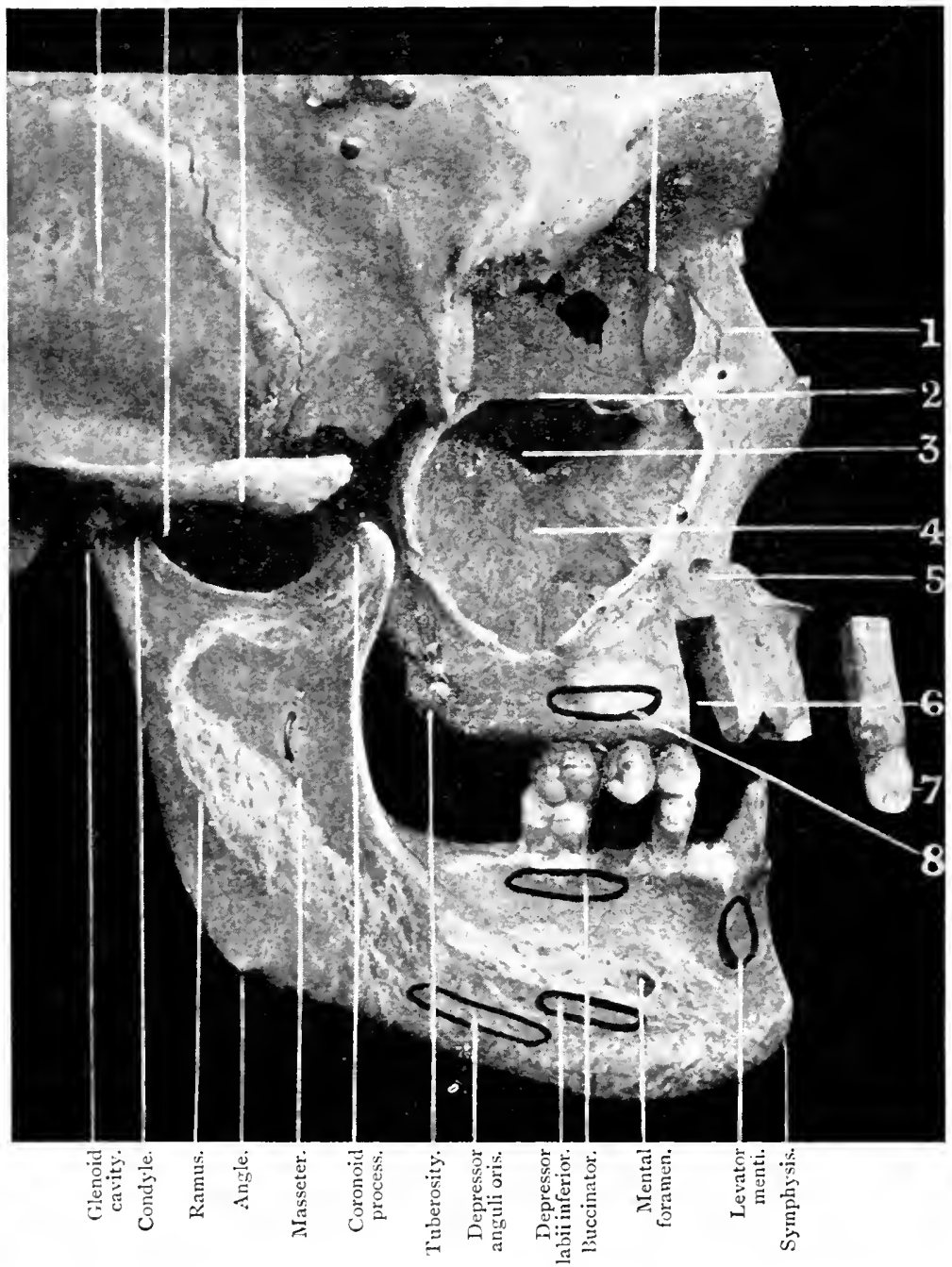

Fig. 34-A General Demonstration of the Main I'arts of the I)ENTAL REGION.

The malar bone and part of the superior maxilla have been removed to show the nasal wall and the boundaries of the antrum. The positions of some of the muscles are also indicated.

I, Nasal process; 2, orbital surface; 3, opening in nasal wall of antrum; 4 , the antrum; 5 , hole bored to show border of antrum; 6 , section to show solid part of bone; 7 , canine section; 8 , buccinator. 
of the coronoid process of the mandible. The deep portion of this muscle is partly concealed in front by the superficial portion; behind, it is covered by the parotid salivary gland. The fibres of the two portions are continuous at their insertion.

The temporal muscle is a broad radiating muscle, situated at the side of the head, and occupying almost the entire extent of the temporal bone. It can be distinctly felt by placing the hand over the temporal region and clenching the teeth tightly together. Its fibres converge as they descend and terminate in a tendon, which is inserted into the inner surface, apex, and anterior border of the coronoid process, and the anterior border of the ramus of the mandible nearly as far forwards as the last molar.

The masseter and temporal muscles are supplied by the inferior maxillary branch of the fifth pair of nerves.

The external pterygoid is a short, thick muscle, somewhat conical in shape, which extends almost horizontally between the zygomatic fossa and the condyle of the mandible. It arises by two heads, separated by a slight interval:

I. The upper from the inferior portion of the external surface of the greater wing of the sphenoid bone, and from the pterygoid ridge which separates the zygomatic from the temporal fossa.

2. The lower from the outer surface of the external pterygoid plate. Its fibres pass horizontally backwards and downwards to be inserted into a depression in front of the neck of the condyle of the mandible, and into the front of the interarticular fibro-cartilage of the temporo-mandibular articulation, which latter part we shall describe later on in this chapter.

The internal pterygoid is a thick quadrilateral muscle, and resembles the masseter in form. Its arises from the pterygoid fossa, being attached to the inner surface of the pterygoid plate and to the grooved surface of the tuberosity of the palate bone, and by a second slip from the outer surface of the tuberosities of the palate and maxilla. Its fibres pass downwards, outwards, and backwards, to be inserted by a strong tendinous lamina into the lower and back part of the inner side of the ramus and angle of the mandible as high as the dental foramen. These muscles are supplied by the inferior maxillary nerve.

The temporal, masseter, and internal pterygoid muscles raise the mandible against the maxillæ with great force. The external 
pterygoids assist in opening the mouth, but their main action is to draw forward the condyles and interarticular cartilages, so that the mandible is protruded and the lower incisors projected in front of the upper ones. In this action they are assisted by the internal pterygoids. The mandible is retracted or drawn back by the posterior fibres of the temporal muscles.

\section{The Importance of the Temporo-Mandibular Articulation.}

A very important part of the muscular mechanism of mastication is the articulation of the mandible, and is called the temporomandibular articulation. This consists of the condyle of the lower jaw fitting and working into its socket, which is the glenoid cavity of the base of the temporal bone. This mandibular joint, which forms a hinge for the lower jaw to work in, while in the act of masticating and articulation, is controlled and held in place by five ligaments:

I. Capsular.

2. Interarticular fibro.

3. External lateral.

4. Internal lateral.

5. Stylo-mandibular.

The joint itself is connected by the capsular ligament, which forms a thin and loose but distinct capsule, attached above to the circumference of the glenoid cavity and the articular surface immediately in front, and attached below to the neck of the condyle of the mandible.

The interarticular cartilage is a thin plate of an oval form, placed horizontally between the condyle of the mandible and the glenoid cavity. It accommodates itself to the form of the glenoid cavity, and acts as a washer between the bony surfaces. Its circumference is connected to the capsular ligament, and is thus contained within it. Its surfaces are smooth and divide the joints into two cavities, each of which is furnished with a synovial membrane.

The synovial membranes, two in number, are placed one above the other below the interarticular cartilage. They provide the synovial fluid which lubricates the joint.

The external lateral, the internal lateral, and the stylo-mandibular cartilages are strong, sinuous cords, which act as auxiliary supports to steady the bones in working. 
The movements permitted in this articulation are very extensive. Thus the mandible may be depressed or elevated, or it may be carried forwards or backwards, a slight amount of lateral movement is also permitted. It must be borne in mind that there are two distinct joints in this articulation: one between the condyle and the interarticular fibro-cartilage, and the other between the fibro-cartilage and the glenoid cavity. When the mouth is but slightly opened, as during ordinary conversation, the movement is confined to the lower of the two joints. On the other hand, when the mouth is opened more widely both joints are concerned in the movement.

The mandible is depressed by its own weight. It is elevated by the anterior part of the temporal, masseter, and internal pterygoid muscles. It is drawn forwards by the simultancous action of the external and internal pterygoids, the superficial fibres of the masseter, and the anterior fibres of the temporal, and it is drawn backwards by the deep fibres of the masseter and the posterior fibres of the temporal muscles. The grinding movement is caused by the alternate action of the pterygoids of either side.

The temporo-mandibular articulation is quite superficial. Its position can be ascertained by feeling for the condyle of the jaw, the movements of which can be distinctly felt in opening and shutting the mouth. When the mouth is opened widely the condyle advances out of the glenoid cavity on to the eminentia articularis, and a depression is felt in the situation of the joint.

\section{Dislocation of the Lower Jaw.}

The mandible is dislocated only in one direction-viz., forwards. This accident is caused by violence or muscular action. When the mouth is open, the condyle is situated on the eminentia articularis, and any sudden violence, or even a sudden muscular spasm, as during a convulsive yawn, may displace the condyle forwards into the zygomatic fossa or space. The dislocation may be unilateral or it may be bilateral. The operation of reduction to put it into place is accomplished by depressing the jaw with the thumbs placed on the last molar teeth, and at the same time elevating the chin. The downward pressure overcomes the spasm of the masseter, temporal, and internal pterygoid muscles, and the elevation of the chin throws the condyle backwards. 
The above-mentioned muscles then draw the condyle back into its normal position.

The parts in close relation to the condyle of the mandible are liable to damage if any great force is applied to the bone, or if any inflammation of the joint may extend to the ear; or, on the other hand, inflammation of the middle ear may involve the articulation and cause its destruction, thus leading to ankylosis of the joint. The joint is also occasionally the seat of osteoarthritis, leading to great suffering during efforts of mastication. A peculiar affection sometimes attacks the neck and condyle of the mandible, consisting in hypertrophy and elongation of these parts, and consequent protrusion of the chin to the opposite side.

\section{The Action of swallowing Food, both Solid and Liquid.}

The act of deglutition or swallowing of the food follows mastication, and is a complex mechanical process which only takes a moment to occur, but it involves the action of many muscles, and any derangement or inflammation of the tissues at the back of the mouth will cause difficult swallowing. As this muscular act of swallowing is always present when a patient is under a general anæsthetic it is of great importance that nothing interferes with the muscles of the tongue and throat. To complete the consideration of swallowing we have to consider the importance of breathing, and to see that the larynx is working satisfactorily, which brings muscular action into play for its own process, and co-ordinates with the pharynx in the swallcwing of liquids or solids.

When the food is properly masticated it is transmitted in successive portions to the stomach by the act of deglutition or swallowing. This, for the purpose of description, may be divided into three acts:

Firstly, particles of food collected form a bolus, which glides between the surface of the tongue and the palatine arch until it has passed the anterior arch of the fauces, which is the hind portion of the mouth terminated by the pharynx and the larynx.

Secondly, the bolus or morsel is then carried through the pharynx.

Thirdly, it reaches the stomach through the œsophagus or gullet. 
These three acts follow each other rapidly. The first act is voluntary, although it is usually performed unconsciously; the morsel of food when sufficiently masticated is pressed between the tongue and palate, by the agency of the muscles of the tongue and palate, in such a manner as to force it back to the entrance of the pharynx. The second act is the most complicated, because the food must go past the posterior orifice of the nose and the upper opening of the larynx without entering them. When it has been brought by the first act between the anterior arches of the palate, it is moved onwards by the movement of the tongue backwards, and by the muscles of the anterior arches contracting on it and then behind it. The root of the tongue being retracted and the laryn $x$ being raised with the pharynx and carried forwards under the base of the tongue, the epiglottis, which is situated just behind the tongue, is pressed over the upper opening of the larynx and the morsel glides past it. The closure of the glottis is additionally secured by the simultaneous contraction of its own muscles, so that even when the epiglottis is destroyed there is little danger of food passing into the larynx so long as its muscles can act freely. In man the epiglottis is not drawn down as a lid over the larynx during swallowing. At the same time the raising of the soft palate, so that its posterior edge touches the back part of the pharynx and the approximation of the sides of the posterior palatine arch, which move quickly inwards like side curtains, closes the passage into the upper part of the pharynx and the posterior nares or basal area of the nasal cavity as it enters the pharynx and forms an inclined plane along the under surface of which the morsel descends. Then the pharynx, raised up to receive it, in its turn contracts and forces it onwards into the œsophagus or gullet leading to the stomach.

The passage of the bolus or morsel of food through the three constrictor muscles of the pharynx is the last step in this stage. The third act of swallowing consists of its passage through the œsophagus to the stomach. The swallowing both of solids and liquids is a muscular act and can therefore take place in opposition to the force of gravity.

In swallowing liquids the two mylo-hyoid muscles of the tongue form a diaphragm below the anterior part of the mouth. The stylo-glossus muscles draw the tongue backwards and elevate its base. The two hyo-glossus muscles act with these, pulling the tongue backwards and downwards. The action of these 
various muscles resembles that of a force pump projecting the mass of fluid down into the œsophagus. There is, however, no hard and fast line between the swallowing of solids and fluids, the more liquid the food is, the more does the force-pump action just described manifest itself.

\section{The Salivary Glands and Saliva.}

During mastication and swallowing of the food the particles are thoroughly mixed and incorporated with the saliva.

The saliva is the first digestive juice to come in contact with the food. The secretions from the different salivary glands are mixed in the mouth, and the liquid is transparent, slightly opalescent, of slimy consistency, and may contain lumps of pure mucin. The saliva is secreted by three pairs of salivary glands:

The submaxillary.

The sublingual.

The parotid.

The submaxillary pair of glands is situated in the angle of the neck at the extreme base of the tongue and just above the voicebox of the larynx. They are round and somewhat thickened bodies. The sublingual glands are situated beneath the tongue, just against the six-year-old molar, and are thick and deep. The submaxillary and lingual glands are connected by Wharton's duct - which is continued along the base of the tongue and empties itself into the mouth just behind the lower central teeth-and placed immediately beneath the tip of the tongue. One mouth of the duct is placed on each side of the tongue frænum.

The pair of parotid glands in the upper jaw are situated between the end of the mouth and the front part of the ear, one on each side of the face. They are triangular in shape, of superficial thickness, and are spread out on the side of the muscles of the face. Each one is widest at the top and tapers downwards until it almost touches the submaxillary gland in the neck. The saliva from the parotid is carried by Stensen's duct along the external part of the face, then penetrates the buccinator muscle at right angles, and empties itself just against the six-year-old molar. It will be observed that the calcium deposit, called tartar, collects on the back part of the lower front teeth and on the sides of the upper six-year-old molars. 


\section{The Mixture of the Saliva from the Three Pairs of Glands.}

The three forms of saliva, secreted by the three pairs of salivary glands, make a mixture in the mouth which is modified according to the nature of the food being masticated. The sublingual saliva is richest in solid matter $\left(2^{\circ} 75\right.$ per cent.), the parotid saliva is poorest in total solids $\left(0^{\circ} 3\right.$ to 0.5 per cent.) and contains no mucin. The submaxillary saliva contains 2 I to 2.5 per cent. The action of saliva is twofold, physical and chemical. The physical use of saliva consists in moistening the mucous membrane of the mouth, assisting the solution of soluble substances in the food, and, in virtue of its mucin, lubricating the bolus of food to facilitate swallowing.

The chemical action of saliva is due to its active principle called ptyalin. This substance can be precipitated by adding alcohol to saliva in a test tube. Its function is to convert all starchy substances into sugar. The starch is first split into dextrin and maltose, the dextrin being subsequently converted into maltose also. Ptyalin acts best at about the temperature of the body $\left(35^{\circ}\right.$ to $40^{\circ} \mathrm{C}$.), and in a neutral medium; a small amount of alkali makes but little difference; but a very small amount of acid stops its activity. The conversion of starch into sugar by swallowed saliva in the stomach continues for a certain time, it then ceases owing to the hydrochloric acid secreted by the glands of the stomach. The acid which is first poured out neutralizes the saliva and combines with the proteins of the food, but when free acid appears ptyalin is destroyed.

\section{The Larynx.}

There is one important part of the throat which the dental operator must always regard with care. We refer to the larynx or that part of the trachea which is built up on the cricoid cartilage. It contains the arytenoid cartilages and vocal ligaments, terminating at the top with the glottis and epiglottis. The thyroid gland is situated on the external part of the larynx. The epiglottis is situated immediately behind the tongue, and is the covering over the glottis, which is the mouth of the windpipe. The glottis is sometimes called the rima glottis or the fissure or chink of the glottis, and constitutes a narrow aperture covered by the epiglottis when one holds one's breath or swallows (see Fig. 30). It contributes by dilatation and contraction to the 
modulation of the voice. The epiglottis is a flap of yellow cartilage placed in front of the superior opening of the larynx, and at ordinary times projecting upwards immediately behind the base of the tongue. During the act of swallowing, however, it is carried downwards and backwards so as to cover and protect the entrance into the larynx. The tubercle or cushion of the epiglottis is a tumescence or cushion of mucous membrane of the lower part of the epiglottis to enable that structure to close the pharynx more accurately when it is depressed. At the base of the larynx we find the cricoid cartilage, which is a ring of gristle forming the top of the trachea or windpipe. Between this base of the larynx and the epiglottis there is situated the arytenoid cartilages. These are two pyramidal bodies which are articulated at their bases with the oval articular substances which exist on the upper margin of the cricoid cartilage in the human larynx. These two cartilages are joined by the arytenoid muscles. The vocal cords are elastic membranes, the edges of which form the side of the glottis. They are attached in front to the thyroid cartilage and end behind in a process of the arytenoid cartilages, and nearly close the aperture of the windpipe. These vocal cords are called the inferior thyro-arytenoid ligaments. In addition to these there are upper false vocal chords, which are not immediately concerned in the production of the voice. These are called the superior arytenoid ligaments. The thyroid bodies or glands are attached to the front and sides of the larynx and trachea, and vary considerably in size. They are sometimes heavier in the female, in whom they become enlarged during menstruation and pregnancy. Each lobe is about 2 inches in length, its greatest width is about $1 \frac{1}{4}$ inches, and its thickness about $\frac{3}{4}$ inch. An enlargement of this gland is called goitre, and seriously interferes with breathing in the larynx, and we must especially be careful about it when administering a general anæsthetic.

\section{Accidents to the Larynx.}

Foreign bodies often find their way into the air passages. These may consist of large, soft substances, as pieces of meat or clots of blood, which become lodged in the upper aperture of the larynx and cause speedy suffocation unless rapidly got rid of or unless an opening is made in the air passage below, so as to enable the patient to breathe. Smaller bodies, frequently of a 
hard nature, such as fruit-stones, buttons, pieces of bone, and -during extraction of teeth, particularly under a general anæsthetic - a tooth, may slip down and find their way through the rima glottis into the trachea or even the bronchial tubes. The dangers then depend not so much upon the mechanical obstruction as upon the spasm of the glottis, which they excite from reflex irritation. If the foreign body is not coughed up by its irritation inside the trachea or the larynx, then inversion of the patient must be quickly carried out, and, failing that, tracheotomy is resorted to as a means of saving the patient from suffocation.

\section{NOTE.}

The following works have been consulted in preparing the outlines contained in the foregoing chapters.

Gray's Anatomy.

Halliburton's "Handbook of Physiology."

Marshall's "Operative Dentistry."

Gorgas' "Questions and Answers for Dental Students."

Holden's "Human Osteology."

Todd and Bowman's "Physiology and Anatomy."

Underwood's "Notes on Anæsthetics."

Quain's Anatomy.

Dowsett's "Dental Surgery Notes."

Underwood's "Aids to Dental Anatomy and Physiology."

Broomell and Fischelis" "Anatomy and Histology of the Mouth and Teeth."

Andrew Wilson's "Modern Physician."

Cunningham's "Textbook of Anatomy."

Lloyd's Encyclopædic Dictionary. 


\section{CHAPTER XI}

\section{THE CAUSES OF INFLAMMATORY AND SEPTIC DISEASES OF THE ORAL CAVITY}

AFTER having examined the anatomy and physiology of the dental region and the parts of the body involved while in a normal state of health, the question reasonably arises: What causes these parts or tissues to depart from their normal condition and thus become diseased? To answer this question we must familiarize ourselves with certain pathological processes. The study of disease and morbid anatomy is called Pathology.

This science examines and investigates morbid conditions and diseases in relation to their causes. Disease is a term used to denote any alteration of the normal vital processes of the body under the influence of some unnatural or hurtful condition, called the morbific cause. If accompanied by change of structure, it is called organic or structural; if not, it is said to be functional. So that we say that the cause of disease arises from some unnatural condition interfering with the normal conditions of health and manifesting itself in a change of structure of the organs and tissues, or in functional derangement.

The study of disease includes:

r. Symptomatology, or the investigation of morbid conditions and symptoms.

2. Etiology, or the causes of disease, the specific agents or causes generating or producing disease.

3. The part or locality which is the seat of structural disease.

4. The nature and extent of morbid alterations, or lesions, or anatomical signs or evidence, of its existence, in connection with its symptoms, causes, and course during life. This constitutes morbid anatomy.

5. Morbid histology, or the elementary constituents of disease products.

The form of disease may be neurotic, dynamic, adynamic, constitutional, malignant, hereditary, cutaneous, etc. 
Physicians are accustomed to treat of the causes of diseases under two classes. One they call predisposing or indirect causes, which includes conditions which would not in themselves necessarily be capable of causing disease, but which might certainly contribute towards it. The other class they term exciting or direct causes. Under the latter head the disease is actually confronted as to its conditions and direct cause.

Diseases are clinically divided into general and local. General are those which exhibit widespread symptoms; local are those which are confined to a limited area of tissues.

Accompanying most diseases there is a process called " inflammation." This process should be well understood by the dentist, because his work involves either its prevention or its dispersion. This is particularly true when we consider the delicate nature of the tissues on which he has to operate (see Chapter IV., p. 46). Inflammation is the process of reaction exhibited by live tissues to irritation. The irritation may be mechanical, thermal, toxic, chemical, electric, etc.; but in order to produce inflammation the irritant must be directly or indirectly continuous in its action. After an irritation ceases to act the process is simply one of repair. The part involved becomes characterized by heat, redness, and pain, owing to a stoppage of function in the microscopic elements of the involved tissues, or to changes in the bloodvessels and blood, and exudation of the liquor sanguinis with permeation of leucocytes or white corpuscles, without rupture of the walls of the bloodvessels, into the contiguous parts; so that inflammation is an extremely complex process with excessive exudation, terminating favourably in what is called "resolution" when the abnormal process ceases and recovery ensues. If the inflammation does not cease it goes on to varying degrees of suppuration, ulceration, induration, and mortification; this latter condition is known as gangrene when applied to soft tissues, and necrosis when applied to bone.

The course of inflammation is characterized by hyperæmia with proliferation of the cells of a tissue or organ and attended with one or more of the symptoms peculiar to its condition. A medical term, or word, ending in "itis" or "ia " means inflammation; ending in " rrhœa," means a discharge or running. The main part of the word signifies the part to which it applies, such as periostitis, gingivitis, ophthalmia, pneumonia, otorrhœa, 
pyorrhœa, etc. The symptoms of inflammation are heat, swelling, and pain, with disordered function. The first cause being irritation, which is usually first manifested in the vascular system, the capillary vessels are stimulated into active contraction and relaxation, causing a more rapid circulation of the blood, inducing warmth with some degree of uneasiness and discomfort. If it continues it becomes severe in intensity, and is called " acute," which is characterized by warmth, sense of fulness, swelling, and increasing pain, caused by the undue volume of blood in the parts pressing upon the nerve filaments of the inflamed tissue in proportion to the expansion of the vessels. If the inflammation does not become acute, but still continues, it is called "chronic"; the symptoms are not so active but more prolonged, becoming less susceptible to the influence of an irritant and with much less pain than in the acute form.

The course of inflammation either ends by resolution, which is the subsidence of the trouble, with the return of the tissues to their normal condition, or it goes on to suppuration, which is the breaking down of the tissues, thereby producing pus. Inflammation is essentially a protective process, closely allied to repair and body growth. It must not be regarded as an evil, but is a process of vital resistance. Pus formation is but a way of ridding the body of the poisons which it cannot neutralize or carry off through the system.

In order to bring this subject closer to our dental area we will associate inflammation with what is called "infection." Infection is always bound up with the subject of inflammation, and we know by daily experience in our dental operating that we come across one or the other quite frequently, especially as pyorrhœa alveolaris is so prevalent. Inflammation caused by infection is due to the successful invasion of a part of the body by micro-organisms. The micro-organisms which produce suppuration are named:

Staphylococcus pyogenes aureus.

Staphylococcus pyogenes albus.

Streptococcus pyogenes.

Micrococcus pyogenes temis.

These microscopic bodies are terrible in their activities and cause suppuration by their action upon the tissues, the leuco- 
cytes or white corpuscles of the blood, and the embryonal cells. Suppuration spreads by the dissolving action of the pus breaking down the tissues, the débris of which, with the white corpuscles, form pus.

Pus is a thick cream-like liquid of specific gravity about Iozo, but varies; sometimes it is thin and mixed with blood, other times it will be found very thick, and sometimes of a cheesy consistency. It is the product of suppuration, consisting of degenerated leucocytes or white corpuscles, living and dead, liquor sanguinis from the blood, granular detritus from brokendown cellular structures, fat globules, and sometimes coagulated fibrin, with many micro-organisms.

The process of septic inflammation is often likened to a battle going on between the blood and the micro-organisms; the leucocytes of the blood having the power of attacking the invading enemies and destroying them, but this power is more or less according to the immunity and resistance of the blood (see Chapter VII., p. 97). In the process of the defence of the leucocytes a rampart is formed by the fixed tissue-cells being excited to a greater proliferation, whereby a wall of cells is thrown around and throughout the inflamed area, which thus tends to localize the infection. It appears that around the invasion of the staphylococcus this wall is accomplished with comparative ease, but around the streptococcus it is not easily accomplished, and it is for this reason that the one remains localized and the other so commonly becomes diffused.

An abscess is a circumscribed cavity containing pus. An empyema is a collection of pus in an anatomical cavity such as the pleural cavity or the antrum.

If the formation of pus continues without being liberated there is danger of serious complications with the blood-stream. The condition of congestion or stasis which accompanies inflammation is an unfavourable factor, because the capillary vessels become so engorged with blood that the circulation at that part becomes blocked and stagnant, thus forming a favourable breeding-ground for virulent bacteria to flourish in.

The complications that attend accumulation of pus through lack of eliminative drainage are termed " secondary infections," and are very serious in their consequences. It is very important that we realize the nature of these infections. If septic inflammation spreads along the blood-stream the condition is known as 
(a) pyæmia or (b) septicæmia, according to its mode of transfer. Pyæmia consists of small infected blood-clots, which are septic emboli breaking away and floating in the blood-stream, being carried away to distant parts of the body, where they may become lodged in the capillaries of a vital organ, such as the lungs, liver, or brain, and commence a new focus of infection. Ultimately a pyæmia may set up numerous infections in many parts of the body.

Septicæmia is caused by bacteria getting into the bloodstream, without blood-clots, but being free are exposed to the germicidal action of the blood and are more apt to be destroyed (see Chapter VII., p. 98). It is a grave and dangerous complication according to the amount of highly poisonous substances which the bacteria secrete and give off. These secretions form the poisonous principles called " toxins."

Apart from septicæmia caused by septic infection from pus, this condition may be easily set up through the introduction of food in which ptomaines or toxins have been deposited by micro-organisms before it was eaten.

The result of this infection is to produce a general diseased condition of the body at large, accompanied by fever, temperature $\operatorname{IOI}^{\circ}$ to $103^{\circ}$, great prostration, disorders of the nervous system and internal organs, tendency to heart failure, delirium, cold clammy skin, and coma, ending in death. Chronic inflammation, if not checked, may lead to hypertrophy, induration, swelling or congestion, atrophy, tumefaction, and ulceration.

Hypertrophy is the excessive growth of tissue by great increase of the cell elements.

Induration is a circumscribed, hard swelling due to an enlargement of individual cells as a result of chronic inflammation.

Tumefaction is a condition due to an abnormal quantity of tissue of a different character from that of the surrounding tissue, especially in colour and texture.

An ulceration is an open surface or sore in a condition of suppuration with no inclination to heal.

An ulcer is the defect that remains after a local surface destruction of tissue from bacterial invasion, from breaking down of a tumour, or following a number of inflammatory processes the cause of which we may not understand. The varieties of ulcer are very numerous. 
We have endeavoured to lay stress on and to accentuate the importance of the dangers attending inflammation, especially with regard to the toxic effects of the ptomaines, or poisons excreted by bacteria.

At this point we ought to have some conception of the nature of micro-organisms, since our work is largely occupied in using antiseptics and methods of sterilization.

Bacteriology is the science of investigating bacteria or living micro-organisms. Bacteria are vegetable organisms so small that they cannot be seen with the naked eye. They live as parasites on the organic matter of animal or vegetable bodies; air, earth, and water teem with them. Our skins are crowded with them, and they swarm in countless millions in our intestines. Fortunately the great majority of species are harmless to man. A great many, in fact, are helpful and necessary in the general economy of life. Professor Metschnikoff, the eminent bacteriologist, has said that " life without microbes is not conceivable at the present day" (see Enzymes, Chapter VII., p. I03). Some bacteria cause fermentation to take place, such as grape-juice into wine, wine into vinegar, malt into beer, ripening cheese, curing tobacco, and a hundred other useful purposes in the industries.

In the examination of the causes of disease in the human body and animal tissues bacteriologists divide microbes into pathogenic and non-pathogenic. The non-pathogenic are more or less harmless under ordinary conditions, but the pathogenic micro-organisms are actual generators of disease, which under suitable or favourable conditions thrive and become virulent.

Most bacteria are aerobic because they live only in the presence of air, others are anaerobic because they live only where air is absent, while a few can exist under both conditions.

The nose, pharynx, and mouth are teeming at any time with bacteria, and they are constantly present in favourably clean mouths. Professor Miller of the University of Berlin, to whom science is indebted more than to any other bacteriologist for our knowledge of the mouth bacteria, made an estimate, in a certain very unclean mouth, that there were not less than I,I $40,000,000$ of micro-organisms present. He also states that nearly all of the pathogenic and many of the non-pathogenic micro-organisms have been found in the human mouth.

While bacteria are vegetable bodies, there are other diseaseproducing microbes which belong to the animal kingdom. 
Among the non-pathogenic micro-organisms are included saprophytic germs. These are sometimes spoken of as " nature's scavengers," from the fact that they prey upon dead and decomposing vegetables and animal matter. These organisms may become indirect causes of disease when they gain access to wounds in which there are pent-up discharges and dying tissues. Under such circumstances they propagate with great rapidity and produce certain poisonous and irritating substances called " ptomaines," which produce similar consequences as we find described under Septicæmia.

The chief difference between the non-pathogenic or saprophytic germs and pathogenic germs is that the former act as indirect causes of disease by the production of poisonous substances which are absorbed by the system, but they have no power to penetrate the tissues or to enter the circulation, while the pathogenic germs possess this power, and act as direct diseaseproducing agents.

Pathogenic bacteria are divided into:

I. Micrococci, or globular-shaped bacteria.

2. Bacilli, or rod-shaped bacteria.

The micrococci live either singly, or in pairs called " diplococci," or in groups of four called "tetracocci" ; others in a chain like a necklace, called " streptococci" ; or like a bunch of grapes, called "staphylococci"; or in a form of a woolpack, called " sarcinæ" (see Fig. 35).

The bacilli, or rod-shaped types, are also classed with curved bacteria known as " vibrios," "spirilla," and " spirochætes."

Bacteria multiply with astonishing rapidity. This process is carried out by segmentation, or the spontaneous division of a micro-organism into sections, each section being complete in itself and in turn subdividing into others; or by gemmation, or the process of proliferation by budding-that is, the growth of one organism out of another by means of spores.

Bacteria require moisture, warmth, and food, for their growth and development, which conditions are very constant in the human body. Fortunately, under healthy conditions and the high resistance of healthy blood, the tremendous rate of multiplication is kept down, otherwise there would soon be an end of our existence. Modern methods of dealing with pathogenic bacteria with antitoxin serums and vaccine therapy, also 

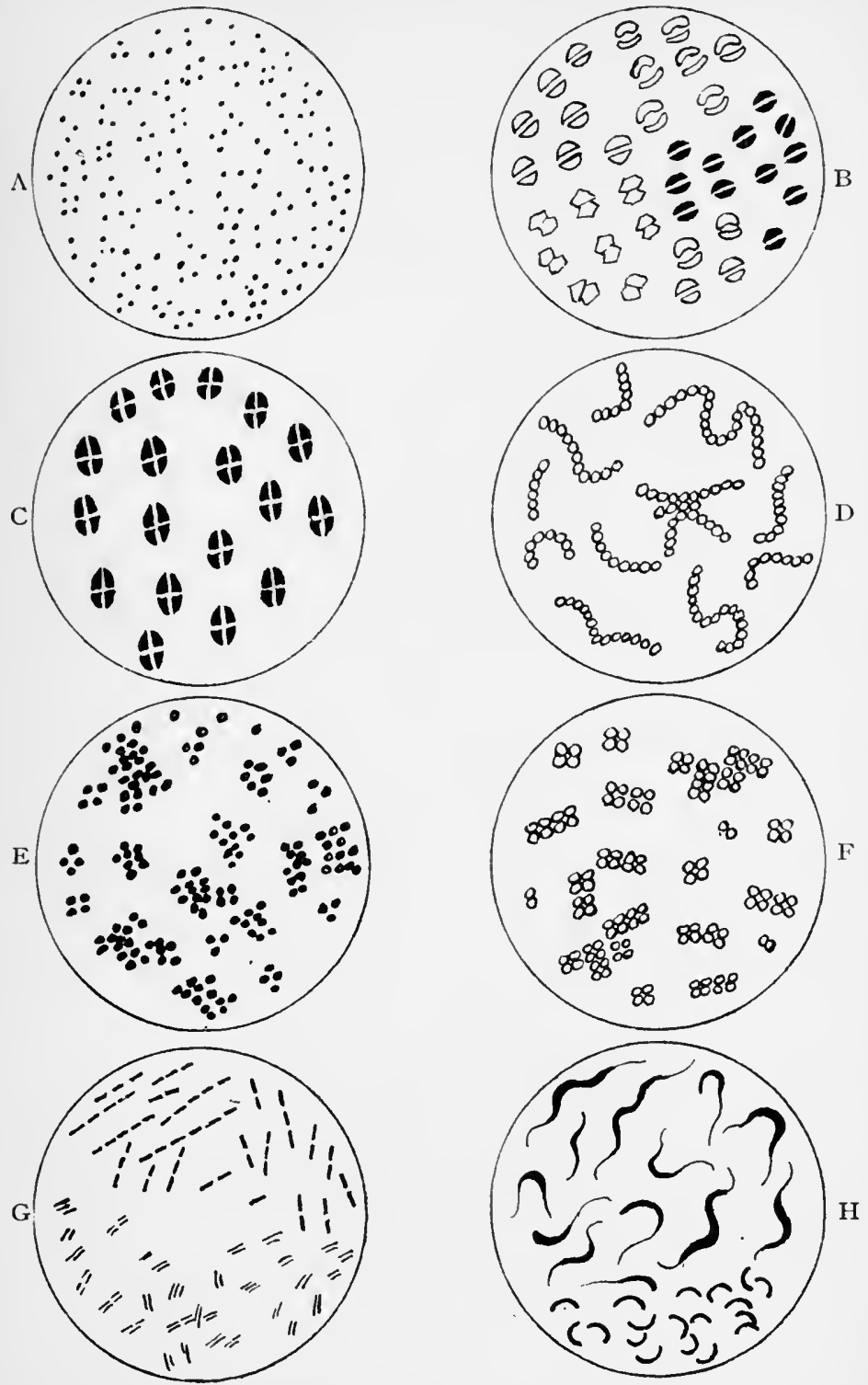

Fig. 35.-Types of Bacteria. (Redrawn from Riddle's " Encyclopædia of Medicine.")

A, Micrococci; B, diplococci; C, tetracocci; D, streptococci; E, staphylococci; F, sarcinæ; $G$, rod-shaped bacilli; $H$, curved bacilli. 
various methods of sterilization with germicidal solutions and antiseptics, keep the danger well in hand. The dangers to be feared are mostly from inability on the part of the patients to realize the gravity of having unhealthy conditions of their mouths and bodies neglected, which give free play to the ravages of these enemies of mankind.

\section{REFERENCES.}

Lloyd's Encyclopædic Dictionary.

Wilson's "Modern Physician."

Blair's "Surgery of the Mouth and Jaws."

Gorgas' "Questions and Answers for Dental Students."

Riddle's "Encyclopædia of Medicine."

Marshall's "Operative Dentistry." 


\section{CHAPTER XII}

\section{DISEASES OF THE ORAL CAVITY}

IT is now necessary that we should acquire a general knowledge of the diseases and morbid conditions of the oral cavity. We propose to put these into a classification with a definition of each condition. It must be understood that our list or classification is a long way from complete or even comprehensive from the oral surgeon's point of view. Also the ramifications and varieties of each disease, according to its importance or significance, would occupy the pages of a very large volume. It does not fall within our sphere of operations to treat them, excepting those which may be regarded as being directly associated with the teeth. But we ought to train our eyes to observe anything in the mouth that is not normal. It is surprising how one can spot any characteristic in the shape of the anatomical parts of the mouth and throat in a normal mouth. This habit will soon lead us to observe small abnormal matters, which will then become a matter of ease in detecting diseased conditions.

The throat, tonsils, soft palate, tongue, floor of mouth, cheeks, and gums, are often affected with some swollen, inflamed, hyperæmic, anæmic, or follicular condition which should affect our judgment in proceeding with our work. Medical men often send patients for dental treatment, especially children, who are affected with gland trouble in the neck, in the neighbourhood of the jaws and ears. If we proceeded with the treatment of the teeth without observation first we might easily make matters worse. The wise course to take would be to make our observations partly by seeing the condition first, and then, by questioning, form our judgment of the condition of the patient in relation to dental treatment. Our knowledge should then help us to form some idea of the nature of the condition and to let the patient understand that we have observed it, but not necessarily to discuss it, because we are not medical men, and the condition might be a passing one or it might be something of a prolonged nature, or even something serious which demanded surgical 
treatment. So that it is not wise to even hazard an opinion, as these conditions are not within our sphere of operations. Again, a patient might be referred for dental inspection and treatment where glandular trouble is present, the medical man not being satisfied as to the cause of the trouble. As the lower jaw is drained by the lymphatic vessels into the neck, there might, of course, be some point of infection existing in the lower teeth. But if we extracted a lower molar straight away, without observation, the swelling might become larger almost immediately and other symptoms intervene which the patient might attribute as the result of the extraction, which might or might not be the case, whereas treatment of a preparatory kind might have averted the after symptoms. Root treatment applied to an upper tooth in the region of the antrum, where antral suppuration might already exist, might cause some worse condition which, had we known, would have guided us in our methods of treatment. In innumerable ways our treatment of the gums and teeth should be subjected to reflection and judgment according to conditions present.

Our consideration will be directed as follows:

(a) Diseases of the throat, tongue, nerves, salivary glands and ducts, jaws, mucous membranes and gums.

(b) Secondary diseases as sequelæ of dental infection.

The throat, or the upper part of it, can easily be seen from the opening of the mouth. The farthest part in view is the pharynx or pharyngeal wall, which is more or less flattened and wide. It is partly hidden by the soft palate and the tonsils. The pharyngeal wall is sometimes in a relaxed and congested condition; and sometimes small, whitish pimples or even patches may be seen. This condition might be a relaxed state of the mucous membrane, or inflammation called " pharyngitis " may be present. In children there may seem to be a congestion and the parts somewhat out of position, which might indicate adenoid growths in the upper part of the pharynx. In adults we might suspect polypus growths in the nose which would possibly cause an obstruction in the nasal passages. This state of the breathing passages might make matters awkward if we applied the rubber dam for filling operations or in nasal anæsthesia. We might also be misled in this condition on finding in our examination that the patient apparently could breathe 
through the nostrils, but during the operation it might turn out that the patient could expire and not inspire, through the obstructions acting as valve flaps in the passages. The tonsils are often in a diseased state. They may be swollen temporarily or they may be in a continued enlarged form, even to almost meeting each other. This state of things amounts to almost complete stoppage under $\mathrm{N}_{2} \mathrm{O}$ anæsthesia, because the air-passage from the mouth would be constricted by the balling up of the tongue against the tonsils and the pressure of the velum of the soft palate downwards on them so as to complete the closure. Inflammation of the tonsils is called " tonsilitis " and also quinsy. There is also abscess of the tonsil. There may also be present a fistulous tract from either the dental tissues or the various parts of the head which form some part of the nasal cavities.

The tongue is an important organ in our observations, as it presents many characteristic signs which may guide us as to a possible derangement of the patient's health. It may be unduly large and show signs of this by deep indentations on its sides caused by pressure on the teeth. Its colour may be modified or exaggerated. Undue redness is often supposed to indicate derangement of the stomach, but this is not always the case. A livid or purple tongue is usually dependent upon deficient aeration of the blood; or it may be very pale, which is a sign of poorness of blood or of great debility. The furred tongue of a yellowish hue is commonly indicative of a disordered liver. A brown colour is usually a sign of a, low state of the system. A smooth, red, glossy tongue is not uncommon in chronic diseases. The papillæ of the surface of the tongue are sometimes large and bright red in colour, and towards the base large round papillæ may occasionally be seen, which probably proceed from stomach disorder of a temporary nature.

Nodules on the tongue indicate many conditions, and may be the early stages of a tumour. Ulcers of many kinds may also be present. Various forms of cancer attack the tongue and may be developed by the irritation of the sharp, ragged edges of a tooth or root. The same cause may also produce septic infection of the tongue if pus is trickling constantly from an infected socket as in pyorrhœa or suppurative periostitis. There are also tubercular ulcers of the tongue.

Syphilis may be seen on the tongue, lips, cheeks, and tonsils. The signs are the chancre, or canker, which appears in two forms, 
the smooth and the ulcerated, and also mucous patches. The first appears as a smooth, round, or oval excoriation, not elevated, sharply outlined and covered with a greyish purulent secretion. It is distinctly hard or indurated, but not ulcerated. The ulcerated chancre is indurated, but the surface shows a shallow, concave, or saucer-shaped ulceration. Mucous patches are usually multiple and rarely appear without other signs of secondary syphilis. Their appearance varies, but they are usually round or oval, almost white in colour, and slightly elevated. They are rarely painful unless ulcerated.

In all of its manifestations, syphilis is due to the infection with the Bacillus spirochata pallida, which is transmitted only by contact. The hard chancre is often situated on the lip. It first appears as a crack or superficial abrasion, surrounded by some induration. The contraction of syphilis by infection might well be a source of anxiety to the dental operator; he is constantly coming into direct touch with patients' blood with his hands, and there is also the danger of splashes into the eye, which is a vulnerable part for receiving infections.

The seventh pair of facial nerves and also the fifth pair, or trigeminal nerves, are the seats of pain which may be present in any part of their trunks and branches; it is called "neuralgia," and is manifested by severe paroxysmal pain in a nerve, apart from the pulps of the teeth. It may be caused by irritation, or by sympathetic action with inflammation of surrounding parts, or by debility, overwork, and general depression. Neuralgia is a term used frequently to cover up an undiscovered cause. This is quite legitimate in itself as a medical term, but there are dozens of causes arising from dentition, impacted wisdom teeth, leaking fillings and crowns, acid conditions of the mouth affecting the necks of teeth under the gums, especially in pyorrhœa and gingivitis, abrasion of plates against the teeth causing tension of the pericementum and irritation, and also acid conditions inside bands and ligatures of plates. Neuralgic cases lay upon the dental operator some degree of responsibility in order to really find a dental cause, and, however conscientious he may be, it will sometimes baffle him, only to be discovered at some future time or by someone else. The study of neuralgia, coupled with practical experience of examination of conservative work in the mouth, is capable of inspiring reflection as to what may 
result from careless or inexperienced practical conservative work. One shudders at the septic conditions of many teeth under crowns and bridges, and one may well add to this the amount of remote pain which passes for neuralgia or odontalgia. The ultimate failure of dental operations may be expressed in neuralgia and septic infection. No amount of pathological knowledge of disease will make up for lack of mechanical skill in operative work, and contrariwise the most exquisite mechanical work must be subservient to the relentless laws which control the inviolability of the nerves and blood-stream. These two vital parts of the body are the themes running through these lectures (see Chapter XX., Root Treatment).

The salivary glands and ducts are liable to inflammation. parotitis, commonly known as "mumps," is an infectious inflammatory disease which may affect one or both parotid glands. Inflammation may be caused in the salivary ducts by deposits inside them. The mouth of Stensen's duct may become irritated by contact with the upper molars, where, in some mouths, we find a large deposit of tartar or salivary calculus. This deposit must necessarily become infected with microorganisms; the one which commonly inhabits this part of the mouth is the Leptothrix buccalis. Stensen's duct is not often affected, but inflammation is often found in Wharton's duct in the floor of the mouth. There may be extensive swelling and severe pain with systemic symptoms. The calculus may form a hard mass within the duct. It is usually caused by some foreign substance getting into it, or by the entrance of the Leptothrix buccalis.

Ranula is a cystic tumour under the tongue due to dilatation of the ducts of the sublingual glands. A sublingual cyst may be mistaken for ranula; it is painless and contains a thick cheese-like material sometimes purulent and offensive.

After outlining the morbid conditions of the oral cavity generally, there remains to be considered the diseases and morbid conditions of the dental tissues. These concern us more directly and demand a further investigation. They consist of the jaws or maxillary bones, the gums, and the mucous membrane. This group presents us with a consideration of the bony foundations and the soft tissues covering them.

The upper and lower maxillary bones or jaws are prone to diseased conditions which are easily developed from apparently 
simple causes. The cause may be simple, such as a blow, or abrasion by food or spiculæ of bone in the food, exposure to chemical fumes, such as phosphorus, arsenic, or the action of mercury, or the entrance at some point by virulent microorganisms, which we term "bacterial invasion." Now these simple causes do not consistently set up trouble in themselves, but depend upon the physiological condition of the patient. There may be latent tendencies to disease in the blood already. The general health, though apparently good, may have some organic structural defects in the tissues themselves. Obviously

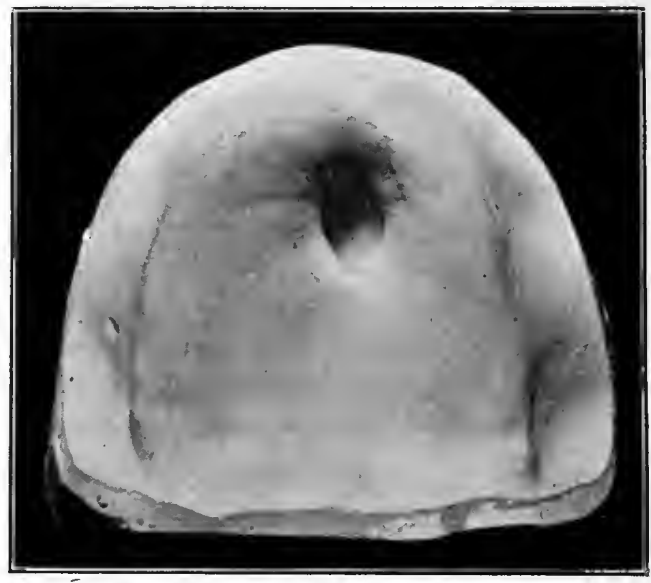

Fig. 36.-Perforation of Palate through Inflammation in the Nasal Cavity.

the resisting power of the blood and the nervous energy of the system must play a very important part.

Necrosis is a serious disease, which ends in the death of the affected part of bone. It is of a similar nature to gangrene or death of the soft tissues. The bone usually passes through the same stages as we find described under Inflammation (see Chapter XI., p. I45). Irritation commences upon the periosteum, inflammation follows, suppuration takes place, the pus having the property of spreading into the bone, the defence of the tissues by the leucocytes takes place, and the trouble is circumscribed or extends accordingly. The pus may work round or circumvent a piece of bone which has not been broken 
down by it; consequently through the cutting off of the bloodsupply this part dies, which in itself may become a source of putrefaction with its danger of producing septicæmia. When the inflammatory process has subsided and the track of suppuration has been arrested, the leucocytes of the blood set to work and separate the dead portion from the live bone. When this has been accomplished it is called " a sequestrum of necrosed bone," which then is gradually thrown off as a foreign body. But in the meantime, before this process is completed, there may be the immediate danger of secondary infections (see end of this chapter). In surgical treatment of bone inflammation in its suppurative stages, drainage is the primary means to be secured for combating it; the use of antiseptics are secondary, but important, of course, to secure asepsis; the leucocytes of the blood and the repairing energies of the periosteum will do the work of clearing it up, but they must be assisted, either locally or through the internal system, by means of suitable medicines, in order to conserve their vitality.

Bone itself is capable of becoming inflamed, and when directly damaged by violence is exceedingly painful. Professor Holden says that "though bone in health has but little sensibility, yet in disease it becomes greatly exalted." Inflammation of bone is called "osteitis." A dental abscess takes place in the bone following infection of the pericementum; this may be a more or less round cavity, small or large in size, which if not checked will form a fistulous tract ending in a fistulous opening. Fortunately, the greater proportion of these cases empty into the mouth near the apices of the roots, but sometimes the fistulous tracts travel in any direction: along the palate, emptying into the throat, causing septic pneumonia, septic gastritis, œdema of the glottis, and pharyngeal abscess, or into the antrum, causing empyema. A fistula is an abnormal tube or canal formed in any part of the body. If the fistula occurs in bone it naturally finds itself resisted, and burrows in the direction of least resistance. In some cases the pus gets directly into the bloodstream, causing a coagulation or thrombus in the veins, and will travel a good way, even as far as the base of the brain. This is a very serious condition. The name of the infection we refer to is "septic thrombosis of the plexus of veins of the cavernous sinus."

The following is a list of secondary infections that might be 
caused by any collection of pus from an infection in the mouth:

Cellulitis of the neck.

Cellulitis of the orbit.

Empyema of the antrum.

Edema of the glottis.

Post-pharyngeal abscess.

Thrombosis of cavernous sinus) By direct infection of

Thrombosis of pterygoid sinus $\}$ the facial veins.

Septic gastritis)

Appendicitis $\}$ By swallowing of pus.

Septic pneumonia, by inhaling septic matter.

Septic and pernicious anæmia.

Lymphadenitis | By infection of lymphatic

Lymphatic abscess $\}$ vessels.

Otitis media, or inflammation of the middle ear.

Mastoid abscess.

Fracture of the jaws is capable of being a serious matter, according to the nature of the fracture and the systemic condition of the patient. It may consist of the breaking away of a small portion of the outer plate of the socket of a tooth, or the cancellous process beneath the surface of the socket. If the patient is in a healthy condition the periosteum in co-operation with the blood will heal and repair the part, if kept in an aseptic condition. The fracture may consist of a clean break or crack running for a short distance and easily held in place by a simple splint, or it may be a long crack, or one running right across the jaws so as to leave a part entirely unsupported, which is a difficult matter to deal with. Or the fracture may be a compound one, with multiple cracks, and portions smashed up. This condition presents a serious complication to be dealt with by a surgeon of some experience in these cases.

\section{Dislocation of the Temporo-Mandibular Joint.}

The articulation formed by the adjusting arrangements of muscles around the neck of the mandible and the glenoid cavity of the temporal bone is sometimes thrown out of gear by accident, either by a direct blow or a fall, or in the course of operating in the mouth by forcing the lower jaw downward. It may be presented as a dislocation on one side only, which is termed 
" unilateral," or on both sides, when it is termed " bilateral." Dislocation consists of the slipping forward of the head or condyle of the mandible which becomes lodged just in front of the eminentia articularis, where it is locked by the varying degree of contraction of some muscles and the overdistension of others, according to the position assumed by the jaw. There are complications with some varieties of dislocation. In an ordinary case the capsular ligament with its synovial membranes will stretch to accommodate the extent of the abnormal position, but, in the case of a fall or a blow, the intracapsular ligament may become dislodged, involving a surgical operation to put the parts into place. In other cases the head of the mandible may be driven into neighbouring bones in some way or other, according to the violence of the cause.

The treatment or reduction of dislocation is usually regarded as being simply carried out by the operator standing behind the patient with his thumbs placed well back on the lower teeth and covered with a napkin, and his four fingers placed under the body of the jaw, then with a downward axis movement, which will depress the head and raise the chin of the jaw, he endeavours to overcome the antagonism of the pterygoid, masseter, and temporal muscles, and at the same time persuade the neck of the jaw with the intracapsular ligament into its place in the glenoid cavity. But there are occasions when the ingenuity of the operator will be taxed to the utmost in trying to get the jaw into place again, and especially if there is some abnormality in the articulation of the teeth which gives no evidence, when open, of its position. While many cases can be put right by simple manipulation, there are some that defy reduction almost. Either one side must go in first and then the other, or both must go in together from the sides or front. In powerfully built men, where all the parts of the temporomandibular joint are fully developed, we can understand the difficulties to be encountered in some cases, involving the necessity for administering chloroform, not for the purpose of relieving any pain, but for overcoming the intense spasm of the muscles and making them as flaccid as possible.

However, the danger of dislocation during operating, in everyday practice, is not very great if care is exercised. We sometimes come across cases where there seems to be a sort of false dislocation. The patient opens his mouth, and in order 
to close it he draws the lower jaw backwards, and with a sudden jerk the mandible closes quickly.

After a difficult case of reduction of dislocation the patient's lower jaw must be kept in a firmly supported position for a few

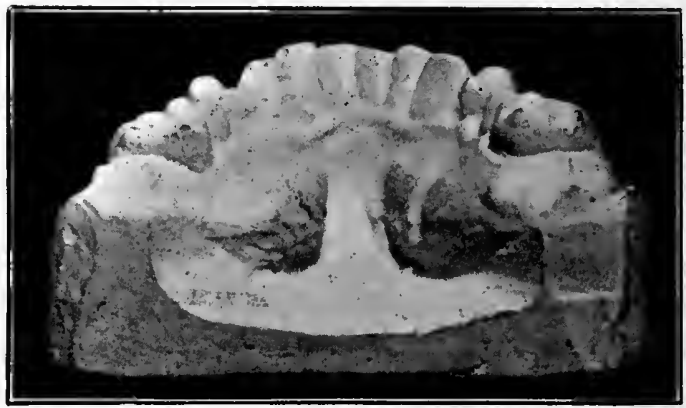

Fig. 37.-Hypertrophy of Bone in Lower Jaw.

days by using a four-tailed bandage in order to give the parts a rest until all risk of possible inflammation passes away, and also to avoid complications with the ear.

Contraction of the muscles of the jaws, making opening and

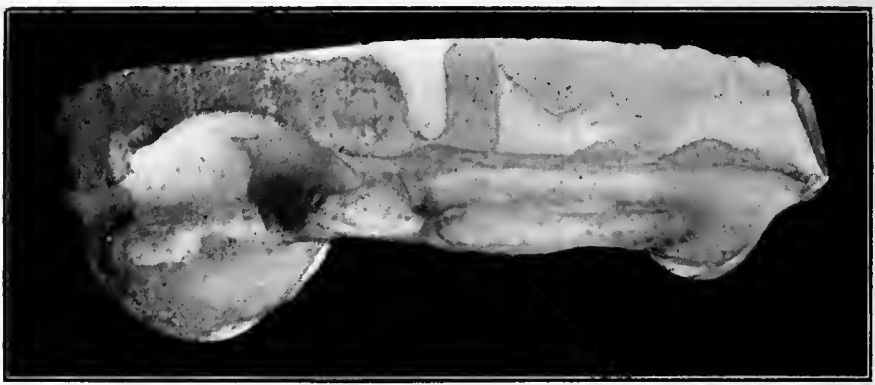

Fig. 38.-Odontoma in Upper Jaw.

(Horizontal View.)

closing difficult, may be caused by inflammatory conditions in the mouth, involving the masticatory or the pharyngeal muscles. Tonsil troubles, an erupting lower wisdom tooth, or any dental abscess, maycause it. Tetanus and hydrophobia will cause lockjaw. 
There are a variety of tumours which appear on the upper and lower jaws. They are usually classed as (a) malignant, and $(b)$ non-malignant or benign. Some are of an inflammatory type, while others may be hard and painless. Many of them attain a large size.

There is also another class of tumour of the jaws called "odontomes"; some of these are formations of tissue such as enamel, dentine, and cementum, mixed up like granite in a

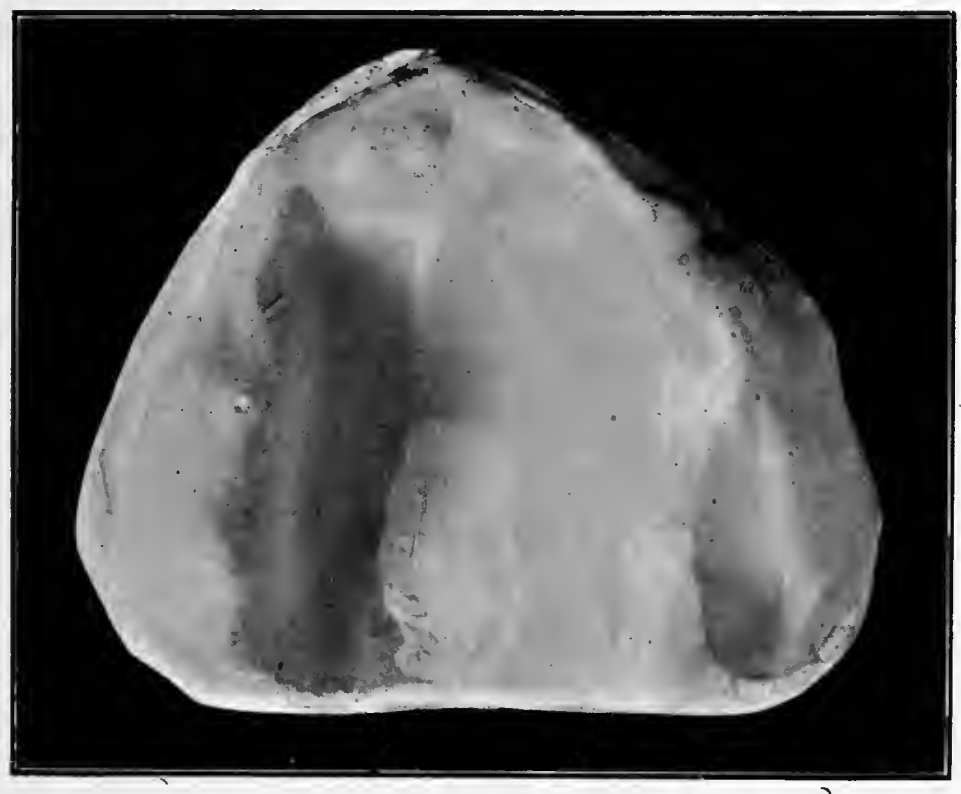

Fig. 39.-Odontoma in Upper Jaw.

(Palatal View.)

tumorous mass, and others are dentigerous cysts containing a tooth or fragments of teeth within them, with fluid or semifluid substance as well.

The mucous membrane and the gums are liable to many diseased conditions :

Stomatitis.

Gingivitis.

Hypertrophy.

Hyperæmia.

Tumours. 
There are several varieties of stomatitis, but there are three main forms.

Follicular stomatitis, which affects the mucous follicles of the mouth.

Ulcerative stomatitis or noma, which attacks the gums and the cheek where it comes into contact with it. It is a serious. and dangerous condition, ending sometimes in sloughing of the soft tissue, and necrosis of the jaw.

Gangrenous stomatitis, or cancrum oris, is a serious disease affecting unhealthy children. It is of a constitutional character.

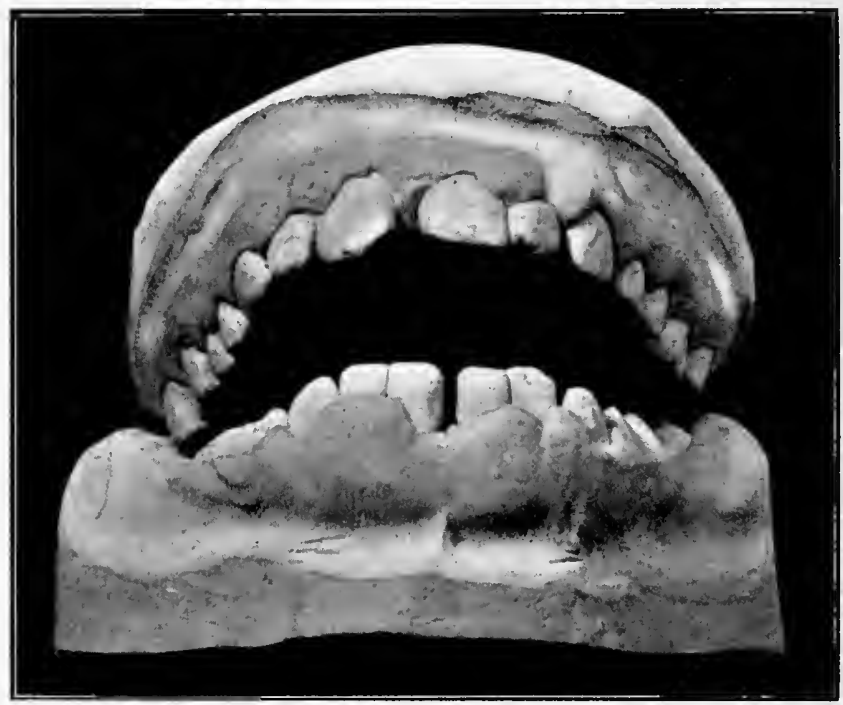

Fig. 40.-Hypertrophy OF Gums.

The cellular tissue of the cheeks are infiltrated with pus and fibrin. The skin is tense and glistening, followed by ulceration and gangrene. It is very often fatal. The treatment must be prompt and energetic by administering internal medicines and the ulcer must be destroyed with powerful caustics such as fuming nitric acid, nitrate of silver, or acid nitrate of mercury.

Gingivitis: see chapter on Children's Teeth.

Hypertrophy of the gums is an excessive growth of normal tissue as a result of chronic inflammation of the gums and mucous membrane. It is caused by an excess of blood, which 
is devoted to the development of extra growths. The papillashaped masses of gum are thick and warty. The treatment of this condition is carried out by using the electric cautery to remove the growth, with the use of suitable mouth-washes.

Hyperæmia of the gums is a mild inflammation which is often seen. It is a deep red or purple in colour with slight swelling.

There are a number of tumours to be found on the mucous membrane and gums. Some are malignant and serious in their condition; others are not so harmful, but must be attended to when active.

Epulis is the name applied to any kind of tumour which grows from the gum. They will grow to a large size in some cases, forcing the teeth apart, and must be removed even if the contiguous teeth have to be extracted to eradicate successfully the roots of the growth.

A polypus of the gum is a smooth, rounded growth, painless and very vascular. It originates from the border of the periosteum. It is easily and successfully removed by the use of the electric cautery, or caustics such as potass. cum calce, or trichloracetic acid (see chapter on Materia Medica).

In Chapter IV., p. 47, we emphasize the importance of the dental periosteum or periodontal membrane and lay stress on its being a constant factor in everything we do in operating on the teeth. It will be better for us to consider the details of the causes of disease of this organ, which are multitudinal, as we come to them in remaining chapters.

There is, however, a disease, which should fall within this chapter, called chronic suppurative periodontitis, which is an inflammation of the dental periosteum. It is commonly met with in some form or other in daily practice. It may be chronic or acute, but differs from a localized dental abscess. It may be caused by a great number of things arising from the teeth themselves, or from details in operative work.

In the chronic form or type, it may go on for years without any great pain, excepting periods of more or less neuralgia which pass off. As time goes along the sockets are subject to a slow inflammatory process, which goes through the same stages as acute inflammation, but is more general than a localized abscess. Small abscesses occur in the area affected around the roots of the teeth at any part of them, thus differing from an apical abscess at the ends of the roots. These small abscesses 
heal up from time to time. The periodontal membrane then deposits, a little more cementum on the root at that spot in order to fill up the space caused by the recession of the abscess. The condition of the root with the added cementum is called "dental exostosis" (see Fig. 4I). An interesting feature of this inflammatory condition is that although the abscess trespassed

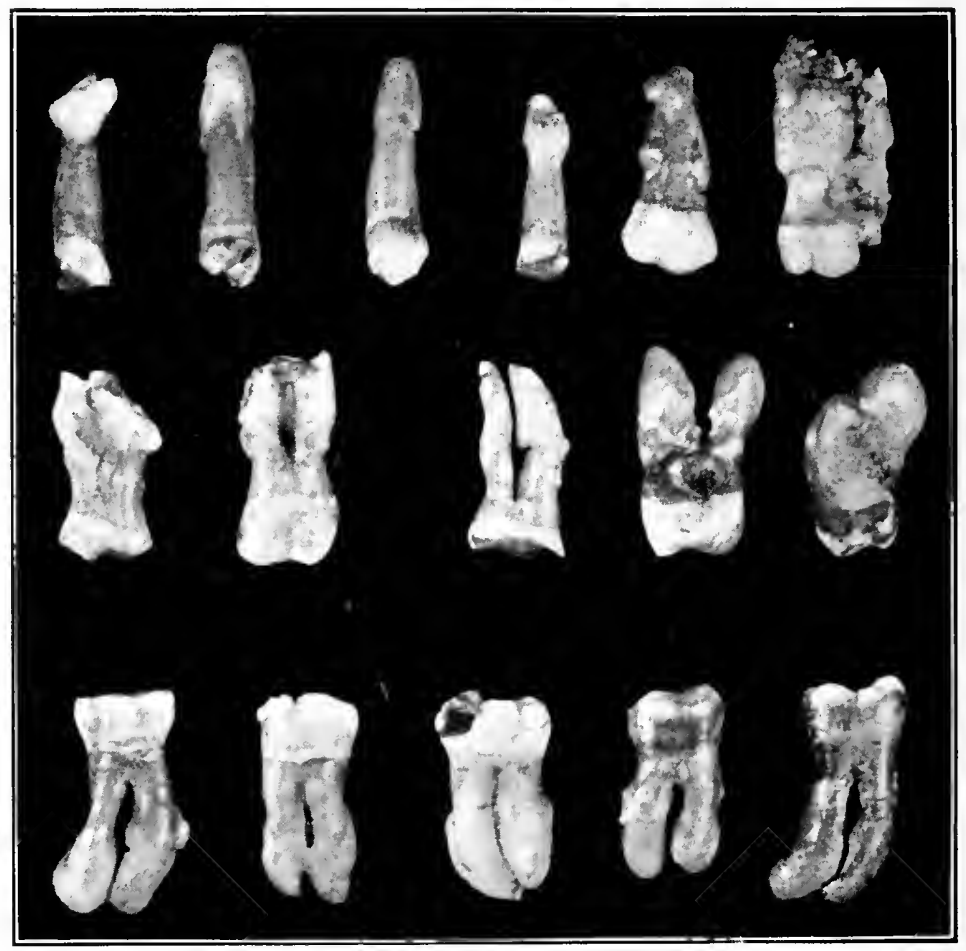

Fig. 4I.-Exostosis of Roots of TeEth.

upon the bone and removed it, yet on the healing and consequent subsidence of the sac, the deposit of the bony substance is upon the root instead of the bone. This condition of dental exostosis is the cause of many a difficult tooth extraction. The tooth may be positively loose and yet defy the efforts of the operator to remove it.

Closely allied to this condition is the disease known as " pyorrhœa alveolaris." This is a general cervical periodontitis. It 
commences at the gingival margins of the gums between the teeth in a similar way to hypertrophy of the gum; it is not a distinct growth, but an inflammatory swelling. These points of gum may be seen with a balling tendency to stand away from the necks of the teeth at the interdental spaces. The next stage

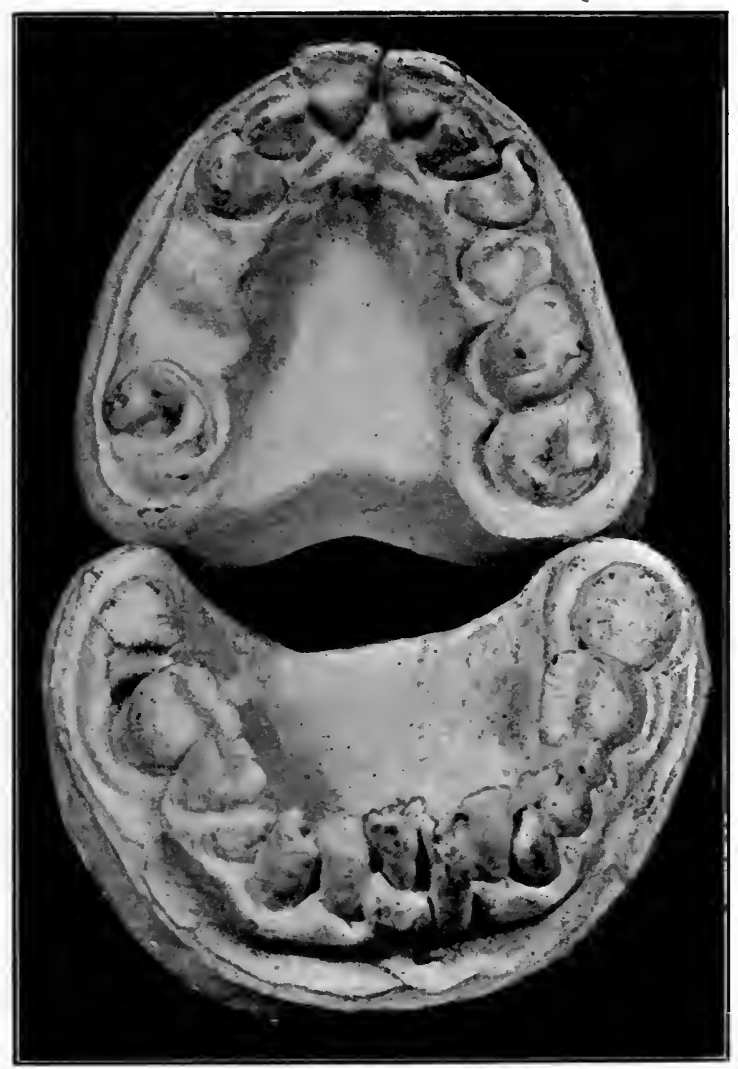

Fig. 42.-Pyorrhea Alveolaris.

involves the centre of the affected tooth, where the disease attacks the socket; this is shown by the deep red colour accompanied by a trace of cheesy-looking pus. In course of time the shrinkage of the gums from the necks of the teeth affected becomes marked, and the thickening of this tissue is greater. Pus pockets are then formed, which burrow down the sockets, 
leading to absorption of the bone, which, becoming infected by putrefying serum and particles of food, and aggravated by further irritation as the teeth loosen, becomes necrotic or necrosed.

This disease is very slow, but progressive. It is not passive or varying like a chronic condition. The worst feature is the constant infection of the general system, which in very advanced and active cases is productive of dangerous secondary symptoms. It is a subject which causes a deal of contention between medical men and dentists. 'Considering that the general system is more important than a part of it, and the teeth being a particularly small part of the general system of organs, one can quite understand the medical man demanding the total removal of any cause of septic infection which is due to the presence of the teeth in the alveoli of the jaws. In fairness to them the $y$ have constantly and daily to undertake the responsibility of saving patients' lives. In order to carry out this responsibility they have to resort to grave and complicated operative measures to vindicate their reputation for skill. One can also quite understand that they have little patience over so grave a condition of obvious infection, when so simple an operation of total removal will completely cure the pyorrhœa. The seriousness of the menace is not to be trifled with.

The dentist is engaged in his surgery, and his patients visit him; he sees them in a favourable condition of health; he may hear of symptoms which have threatened the patient, and after consultation, he decides to extract the affected teeth. The dental profession are justified in placing a very high value on the teeth themselves; it is their work to save and preserve them if possible. It is a matter of very deep concern and regret to them to have to remove many of the beautiful and perfect specimens of teeth thus sacrificed through this prevalent disease. Our experience is that the more perfectly shaped and the stronger the types of structural substance, the worse is the accompanying pyorrhœa.

The public must become more interested and alarmed themselves as to the condition of their teeth and sockets; so many of them are concerned about the appearance and usefulness of the crowns only. Some become anxious on hearing the serious experience of others. However, they leave matters affecting their health almost entirely to their medical advisers. 
Now medical men constantly face the realities of these cases of secondary infection, which we have already mentioned in a list, and in dental books they look quite harmless, as they do not often turn up in dental practice. Let there be no mistake, they are potentialities which only medical men can realize, because they have to wrestle with these serious cases daily, not only from dental causes, but all kinds of causes generally. They are skilful men, and carry their responsibilities with ease, but they have learnt from bitter experience of the terrible activities of the streptococcus. In these cases there is no playing with time, the enemy of the patient's health must be grappled with and removed by some means or other as quickly as possible. So we can understand their attitude towards pyorrhœa and suppurative periodontitis, especially where it is only suspected under a crown or bridgework. Some straight heart-to-heart criticisms have appeared in the Dental Cosmos* during the last few years with reference to these conditions, written by eminent oral surgeons in America, and their indictments have extended to the medical profession in this country. This is significant, and indicates that the dentist must be ready to let the medical man take the responsibility of the wisdom of retaining doubtful foci of infection in a patient's mouth. In the Dental Cosmos, March, I920, p. 371, there appears an article by Robert Burns, Jnr., D.D.S., entitled, "Why Pulling Teeth Fails: A Plea for their Surgical Removal when Evulsion of these Organs is Indicated by Infection." In this article Dr. Burns goes farther than mere extraction of affected teeth; he proposes the necessity of the surgical removal of the teeth in order to eradicate alveolar infection. He says: "Teeth that have long been infected with pyorrhœa, where the pockets are deep and the alveolar process largely gone through infection, should by no means ever be 'pulled,' no matter how loose they may be. In such cases close study of good radiograms will give an idea of the amount of infection and the destruction wrought in the bone. Simple extraction only by greatest good luck would rid the field of this tissue." He concludes by saying: "We are prone to underestimate the dangers of dead (or infected) teeth, and finally to treat their removal as a small matter rather than to attach

* See Dental Cosmos, March, I920: "The Significance of Pulpless Teeth in Health and Disease," by B. B. Machat, D.D.S., Brooklyn, New York. 
thereto the same significance that the removal of dead infected bone would demand elsewhere in the body."

The main principle in determining the retention of teeth with pyorrhœa, or suppurative periodontitis, is the consideration of the probable degree of resistance and immunity of the patient's blood as the deciding factor in retaining dead teeth for conservative treatment, or the retention of a morbid pericementum in pyorrhœa. Dental surgery cannot be an exception to the principles of general surgery.

However, the whole combined medical and dental world are busy in collaboration, endeavouring to find out a solution of the pyorrhœa problem. There is no doubt that there are specialists who do understand the condition, and treat it by using amœbicides and bactericides together with thorough surgical curetting; but this is really a specialist's task, and one can see a probability of its permanent success if thoroughly done in this way. But it is a matter of conviction whether superficial treatment ever touches the foundation of the disease. It may improve for a time, until the patient loses interest, when it gradually gets the upper hand again. We refer to this subject again in the chapter on Dental Materia Medica and Therapeutics.

\section{REFERENCES.}

Blair's " Surgery of the Mouth and Jaws."

Wilson's "Modern Physician."

Dowsett's " Dental Surgery Notes."

Gorgas' "Questions and Answers for Dental Students." 


\section{CHAPTER XIII}

\section{DENTAL MATERIA MEDICA AND THERAPEUTICS}

BEFORE examining the various processes of filling cavities and procedures in the dental surgery, we propose to describe the various drugs that are employed for dental purposes.

The origin and characteristics of drugs come under the subject of Materia Medica.

The compounding of drugs is termed "pharmacology." The application of drugs and their action on animal tissues in health and disease is called "therapeutics."

Therapeutics deals with the form of drugs, the methods of application, and time that they should be administered. It includes a knowledge of the incompatibility of drugs one with another, and also a classification or grouping of drugs according to their action on animal tissues.

So that the drugs we employ should be defined, as to what they are, whence derived, what their appearance is, what their action and dosage, what changes are produced in tissue by their action, and also the nature of the action itself, which is called "pharmaco-dynamics."

The utilization of the action of drugs is for three purposes-the prevention, relief, and cure of disease. The indications for applying or administering drugs in order to employ their action depends on :

The nature and seat of disease.

The symptoms present.

The condition of the organs.

The nature of the parts affected.

The methods employed are-internal administration remedially by the mouth and rectum, internally by inhalation, local and external application, hypodermic injection, and electricity.

The process of the action of a drug in itself-that is, the pharmaco-dynamic forces going on to produce the specific action on tissues--is known only in a few instances. 
There are two factors always involved when studying their action :

I. The power possessed by the drug.

z. The reactive power possessed by the organism.

The particular powers possessed by a drug are theoretically unalterable according to the constancy of chemical laws, but there are factors which may modify them. Chloroform is altered by long exposure to light. Tincture of iodine will develop hydriodic acid and acetic acid. Carbolic acid on long keeping will turn red owing to the development of rosolic acid by absorption of carbonic acid and oxygen. But, provided that drugs are pure and true to their derivation, these alterations are not serious as a rule. But what is important is that all drugs should be of first-class quality. There are so many ways of adulterating them that it is imperative that we secure good quality drugs in order that the small quantities which we use will react to what we expect of them. They should be strictly kept in wellcorked or stoppered bottles, and so protected that neither air, damp, nor fumes can act on them, or heat and light.

The reactive power which is expected might be modified by alteration of the tissues. They might require either stimulation or depression. Factors are also present by age, individuality, idiosyncrasy, and sex.

Drugs act on the cell in three ways:

I. There is a superficial combination of the cell wall with the drug substance which lasts as long as the cell is active and is not injured; the drug substance does not enter the cell itself.

2. A combination of the cell contents with the drug substance which is produced as a result of the easy penetration into the protoplasm proper.

3. An intermediate action which takes time, from minutes to days, in which to react.

Some drugs have a selective or specific action, such as alcohol on the brain, strychnine on the spinal cord, arsenic on the dental pulp, anæsthetics on nerve and brain cells, etc.

The science of chemistry, which deals with the bases, compounding, and manufacture of chemical substances, classifies them by grouping according to their molecular construction and chemical affinities, but in therapeutics all chemicals which are used as drugs are classified according to their general action. 


\section{Classification of Drugs.}

Adjuvants.-Substances which assist in the action of the principal drugs.

Anæsthetics.-Drugs which produce general insensibility to pain.

Anæsthetics, local.-DDugs which produce insensibility to pain in a localized area of tissue.

Analeptics.--Restorative drugs.

Analgesics.-Drugs which allay pain.

Anodynes.-Drugs which relieve pain.

Antacids.-Drugs which neutralize acids.

Antiseptics.-Drugs which inhibit or check the growth of micro-organisms.

Anti-spasmodics.-Drugs which relieve nervous irritability and spasms.

Anti-toxins.-Defensive proteins developed in the body as a result of the inoculation of a poison and acting as a neutralizer.

Astringents.-Drugs which produce contractibility of tissues and arrest discharges.

Cardiac stimulants.-Drugs which increase the heart's action. Caustics.-Drugs which destroy living tissue.

Counter-irritants.-Substances which by counter-irritation relieve some other irritation.

Deodorants.--Drugs which destroy foul odours.

Disinfectants.-Drugs which chemically destroy and render infectious material sterile or inert.

Escharotics.-Substances which produce caustic effects.

Hæmostatics.-Drugs which arrest hæmorrhage.

Irritants.-Drugs which cause irritation.

Neurotics.-Drugs which act on the nervous system.

Obtundents.-Drugs which alleviate pain locally by partial anæsthesia.

Prophylactics.-Substances which prevent the contraction or development of disease.

Specifics.-Drugs which have a direct curative influence on certain specific diseases.

Stimulants-Drugs which increase functional activity.

Styptics.-Local hæmostatics.

Topics.-Local applicators. 


\section{Description of Drugs used in Dentistry.}

Peroxide of Hydrogen.-This chemical may be prepared by the action of diluted sulphuric acid on barium peroxide in presence of water. It is prepared in $10,20,30$, and Ioo volume strength. Ten volumes indicates that there are Io volumes of available oxygen. It may also be produced naturally in many ways. Dose: Io volumes, $\frac{1}{2}$ to 2 drachms. Its action for dental purposes is to remove pus, which it does by a frothing or bubbling process. This is a very useful chemical, because it will fetch matter away from otherwise inaccessible places, such as pyorrhøea pockets and under badly fitting crowns. It is usually employed in Io or 20 volume strength, but is sometimes used in roo-volume strength for root treatment and bleaching cavities, but as it is very caustic it has to be used accordingly.

Its uses are for swabbing (Io volumes full strength) around the necks of teeth after scaling; before filling a cavity, following it with absolute alcohol; and before fixing a crown, because the cement used will probably drive septic matter down, especially between teeth. Some operators use it for removing pus from the apices of canals in root treatment, but this must be done with great caution as there is a risk of the gases getting shut in by a flap of débris. There is really no advantage in using it high up the root because after the first dressing the pus can usually be syphoned out with paper points. Some operators also apply a swab to cleanse a septic socket after extraction; this too is unwise, as there have been cases of septic absorption by gaseous pressure through its use.

For patients' own use a solution of peroxide of hydrogen, Io-volume strength, I teaspoonful to 3 of water, makes an excellent mouth-wash in cases of gingivitis and pyorrhœa, also where any septic surface requires cleansing. It should be used every morning before a meal. In some mouths there seems to be an acid reaction on the teeth; if the patient complains of this, make it every other morning. Its use is advisable before applying any other mouth-wash or a dentifrice because, if the parts are cleared out, the antiseptic can be carried in to its place.

It is not a powerful germicide, but is a good antiseptic. Its chief property is its power of removing pus, and also of attacking blood in the same way; hence its use as a mouth-wash should 
be discontinued for quite a fortnight after extraction of teeth, as the thrombus or blood-clot which becomes part of the repairing process may be removed and a dry socket result.

Absolute Alcohol.-This drug is useful for cleansing the surfaces of teeth and drying out cavities, also as an obtundent of sensitive dentine dried with hot air, or mixed with other drugs. It is a solvent of many substances, and is used in tinctures for this purpose.

Adrenalin and Suprarenin.-This is the active principle of the suprarenal gland. It was first isolated by Takamine. The function of the suprarenal gland appears to be to maintain a proper-degree of contraction of the arteries in order to correct blood-pressure. Adrenalin chloride, which is prepared from the natural gland, suprarenin chloride, a synthetic product, and other suprarenal preparations, are used in medicine as powerful vaso-constrictors. It is so potent that even in weak solutions it must be used with caution, as patients vary in their toleration of it. The adrenalin solution supplied by Messrs. Parke, Davis and Co., is I grain of adrenalin chloride in I, 00o minims of sterilized water with chloretone. It is used in cocaine, eucaine, and novocaine solutions for local anæsthesia. For injection purposes in extraction of teeth $I$ in 50,000 is generally used, but many practitioners prefer I in I00,000 as being more suitable and secures the desired effect. It is employed as an adjuvant, its action being vaso-constricting, and localizes the action of the local anæsthetic, securing a better anæsthesia. The reason for using a weak proportion of adrenalin is that as it is such a powerful vaso-constrictor, if the sockets of the teeth are septic, the condition of stasis that follows and which sometimes persists for some hours afterwards favours the breeding and multiplication of micro-organisms, which will cause further infection, especially in the lower jaw in the wisdom region, as there is less chance of drainage than in the upper. This drug is also used as a valuable styptic: a small plug of cottonwool is saturated with it and well plugged into the socket. but a convenient and better form for plugging is the adrenalin gauze and tape.

Perchloride of Iron is used as a hæmostatic (dose 5 to I 5 minims), or as a styptic by saturating a piece of cotton-wool and packing it into the tooth socket. It is powerful in its action, but is easily washed away because, being a solution, it is squeezed out 
as it enters the socket and is washed away with the saliva. It is also an undesirable agent in the mouth, as it attacks the enamel of the teeth and turns it a dark cocoa-brown colour which cannot always be removed.

Tannin or Tannic Acid is a hæmostatic, styptic, and astringent. It is administered as a hæmostatic in 5 to Io grain doses. As a styptic it is applied on cotton-wool which has been dipped in carbolized resin. It acts by coagulating albumen.

This chemical, which is in the form of fine fibrous crystals, is of a gummy nature when moistened. It is a powerful astringent and tanning agent, and is largely used in tanning hides into leather, irrespective of thickness. Even thick bull-neck hides are successfully tanned with it. Tannin enters into chemical combination with animal tissue and cannot be separated afterwards. The mummifying action which is demonstrated on such a large scale in manufacturing leather can well be utilized as a dental dressing where the amount of tissue is so small. In the six-year-old molars, where it is undesirable to devitalize the pulp because of the ends of the roots not being complete, the soft tissue can be utilized as a pulp cap instead of removing it. Direct pulp capping is not very successful; it may be so in a few cases, but the pulp invariably slowly dies. In the case of incomplete roots there is danger of the arsenic getting out at the ends and setting up pericementitis or even necrosis. Past experience has shown that the majority of direct capping cases come back after a time with septic trouble owing to the death of the pulp. Tannin mixed with pure cinnamon oil is a valuable dressing for tanning soft decalcified dentine. Take 2 drachms of tannin and place on a glass slab and then spatulate a little cinnamon oil with it gradually. Cinnamon is the only oil that will enter into complete combination with tannin. Cassia and other oils will mix with it; the tannin is visible as a mixture only, but with cinnamon it becomes homogeneous. If, after standing for some hours, it becomes too thin, then spatulate some more tannin into it. Warming it over a water-bath makes it better still. It takes very little oil to dissolve a large amount of tannin; experience in mixing small quantities will prove this. When finally prepared it should be of the consistency of soft wax. It can be put away in a small ointment pot ready for use. If it should become hard by long keeping, it can easily be cut with a warmed spatula. Even the 
warmth of the mouth melts it when in this condition. To apply it to a cavity, cut a tiny piece of card, such as a thick visiting card, and spread a small portion of the paste on to it and carry to the cavity, applying the paste side to the dentine. Seal it in with nastic or gutta-percha, leave for a few days or a week, and apply again if it is a bad case, or apply a neatly shaped cap and leave it in place to be covered by cement or amalgam directly placed over it. There is no pain or irritant action from it. If pain should develop after having filled the cavity, it is clearly through not having covered the area of the pulp so as to insulate the thermal conductivity of the amalgam, if used, or from pressure in filling.

Tannin mixed with Dentalone or Dr. Buckley's phenol compound is very useful as a drying or toughening dressing after arsenic has been applied to a pulp.

It is also used mixed with eau de Cologne or alcohol and applied to pyorrhœe pockets as an astringent.

Essential Oils.-There are several kinds used in dentistry, such as cinnamon, eucalyptus, cassia, thyme, geranium, ylangylang, clove, peppermint, gaultheria, cajuput. The last named and eucalyptus are solvents of gutta-percha.

Essential oils are volatile, and on being kept exposed to the air become thick and sticky. Their volatile properties depend upon the presence of gases which they contain, some being more volatile than others.

Cinnamon Oil is a potent germicide. It also has great penetrating power. It is a reliable antiseptic in cavities at the back of the mouth and is not irritating. A suitable lining for deep cavities at the back of the mouth may be made by mixing a tiny drop of the oil with oxy-phosphate of zinc cement.

Cinnamon oil is very useful and reliable in root treatment as a dressing for septic canals, but if any free oil should escape at the apical ends of a root, it will irritate the periodontal membrane causing much pain. It acts by liberating cinnamic aldehyde, which forms a large part of the oil. On account of the discoloration of teeth in front of the mouth some operators prefer to use cinnaldehyde, which is colourless, and is derived from cinnamon oil, as Eugenol is taken from clove oil. The aldehyde property of the oil may well be utilized in root treatment providing it is controlled. This can be done by using fibres of asbestos, or wisps of bibulous paper just 
moistened with it. As a root filling it may be mixed with pure calcined zinc oxide. The oil has a peculiar effect on zinc oxide, it appears to have a fusing action on it; a very small drop will take up a lot of the oxide and makes an excellent cement for plugging the canals. It will set in time. It is essential that we secure the right quality of cinnamon oil for mixing with the calcined zinc oxide, and also for mixing with tannin as a decalcified dentine dressing (see tannin). It seems a difficult matter sometimes to get the right kind, and there seems to be some confusion in the drug trade as to the importance of the difference between the cinnamon oils on the market and oil of cassia. The writer has used the root filling and the capping paste constantly for many years, and has had, once or twice, to discard the so-called pure cinnamon oil because it failed to act as desired. The best oil is obtained from the bark of the Ceylon plant. The oil from the leaf is inferior. The oil from the Chinese plant is dark, and is known as oil of cassia. Oil of cinnamon has the credit of causing bad discoloration in teeth where it has been used, but our experience does not verify this. The pure oil is light in substance and also in colour. The inferior oils are thicker and darker. Cinnamon oil mixed with iodoform is a good dressing for root canals, and is very persistent.

Oil of Cassia mixed with equal parts, by weight, of phenol crystals, is a good antiseptic for back teeth cavities, non-irritating and obtundent. Dr. Black's I-2-3 for putrescent root canals is worthy of attention; it is efficient and non-irritating. (See chapter on Root Filling.)

Dentalone is a valuable dressing as a sedative for inflamed pulps; it will be successful where other drugs have failed. Its ingredients are chloretone crystals in oils of clove and cassia with methyl salicylate. (See chapters on Cavity Preparation and Devitalization; also Chloretone.)

Chloretone is useful as an analgesic dressing; it is soluble $I$ in 200 of water, I in Io of glycerine, I in $5^{\circ}$ of liquid paraffin, I in $\mathrm{I} 2$ of olive oil. It is the main drug in Dentalone, which has been mentioned under oil of cassia. Chloretone inhalant is also very useful for painful sockets a few days after extractions. It is a solution of chloretone, oil of cinnamon, camphor, and menthol, in liquid petroleum oil. It is useful as an anodyne dressing in pericementitis in root treatment. Is useful also as a suitable antiseptic lubricant for hypodermic syringes. 
Eugenol is the active principle of clove oil, of which it should contain at least 85 per cent. It is a useful drug as an anodyne. It can be used for cavity dressing and root treatment. It can be mixed with oxide of zinc to form a paste for lining cavities, especially in children's teeth. It does not set at once, but does so in a few hours, and the saliva will not affect it. It can be covered with cement straight away. Adhesion of the oxy-phosphate of zinc cement will take place better than with cement alone. It can also be used in mixing oxy-phosphate of zinc cement by adding a small spot to the mix, and using it as a cavity lining. If a cavity is swabbed with it and barely dried, it will cause the cement to hold more firmly in those cases where it is difficult to get much undercut.

Chloride of Calcium is employed as a powerful hæmostatic in cases of suspected hæmophilia, or cases where the patient has previously had severe secondary hæmorrhage. It is administered in the form of tabloids (dose 5 to 15 grains, three times a day) for a few days or a week before the operation, and continue, if necessary for a day or two after. There is a possibility of blood clotting in the bloodvessels by too long use in certain types of people. Lactate of calcium may also be used for the same trouble instead of the chloride.

Calcium Sulphide.-Dose $\frac{1}{4}$ to I grain. It is largely used in medicine for boils, carbuncles, and scrofulous sores, especially in the glands of the neck. In abscess of the ear, or wherever there is pus formation, calcium sulphide will act beneficially. In follicular tonsilitis $\frac{1}{2}$ grain may be given every hour until alleviation, then every two hours. It is said to act as a specific. In the treatment of contagious diseases of childhood calcium sulphide seems to act similarly to antitoxin- that is, it increases the inherent power of the blood to destroy bacteria and their poisons. In periostitis and alveolar abscesses it has been found of service. Stearettes of calcium sulphide freshly made should prove useful in suppurative conditions in fairly large doses, say I grain in each. In this form they would avoid decomposition in the stomach. The aperient action on the bowels must be watched. (From Martindale and Westcott's Extra Pharmacopeia on caix sulphurata.)

Phenol or Carbolic Acid is a powerful, antiseptic escharotic, and anodyne, mixed with glycerine. It is extensively used in dentistry. As a cavity dressing it must not be used in the 
crystal form or the 85 per cent. liquid form, as its action coagulates albumen. The effect of this produces an escharotic sealing of the dentinal tubes by attacking the nerve fibrils within them, and closing micro-organisms in the tubes without destroying them. Also the escharotic coagulation of soft tissue where a pulp is partially exposed will preclude the possibility of applying another drug with effect, such as arsenic, because it cannot penetrate the coagulum.

The coagulating property of carbolic acid can be removed and its valuable antiseptic action retained by mixing it with other chemicals. Dr. Buckley advocates a useful formula:

\begin{tabular}{|c|c|c|}
\hline Liquid carbolic acid & - & 3 fluid drachms. \\
\hline Thymol crystals & . & $4^{\circ}$ grains. \\
\hline Menthol crystals & $\cdots$ & $\ldots$ \\
\hline
\end{tabular}

Phenol crystals may be mixed with essential oils (see Cassia) or with glycerine. If equal parts of carbolic acid crystals with thymol, menthol, or camphor crystals are placed in a stoppered bottle they will become liquefied and are very useful as antiseptic dressings for front teeth.

Carbolic acid is a good mouth-wash for syringing out septic sockets after extractions, especially when a local anæsthetic has been used and the socket is very painful; I in I2O in hot water is suitable (see chapter on Extraction of Teeth).

Phenate of soda and Bobœuf's Phénol Sodique are invaluable for syringing painful sockets after extraction of teeth, but they are not suitable where local anæsthetics have been injected on account of their containing alkali which might be free in the solution.

The dose of carbolic acid is I to 3 grains. It is very poisonous.

Zinc Sulpho-carbolate is recommended by Whilslar for treatment of pyorrhœa pockets in a Io per cent. solution which he injects deeply with a pyorrhœa syringe. Messrs. Parke, Davis and Co., supply tabloids of this compound, each one containing 5 grains, so that ten tablets dissolved in I ounce of cinnamon water give a Io per cent. solution.

Oil of Gaultheria or Wintergreen is a useful antiseptic on account of its anodyne property. It may be used in root treatment. It should contain 99 per cent. methyl-salicylate.

Methyl-salicylate may be used with an equal part of carbolic crystals as a colourless antiseptic for cavities in front teeth.

Carbolized Resin is a useful emergency dressing. When a patient has a painful tooth with pulpitis and the operator is 
busy, a pellet of wool dipped into the resin will sooth the tooth until it can be properly dressed. It is prepared by mixing:

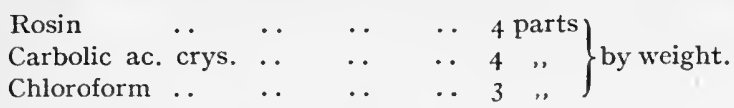

This preparation is very useful as a styptic in conjunction with the astringent property of alum. It has the advantage of fixing the styptic where it is required, as the carbolized resin sets when the chloroform has volatilized, and, with the cottonwool carrying it, forms a hard plug which has the further advantage of being antiseptic, whereas cotton-wool and gauze are apt to become foul in a short time.

Para-mono-chlor-phenol is prepared by passing chlorine into phenol. It comes in the form of needle-shaped crystals. Merck of Darmstadt is a reliable manufacturer of this product. The crystals should be separate. It is practically insoluble in water, although the crystals appear very moist, but soluble in glycerine, alcohol, and essential oils. It may also be rubbed or spatulated with vaseline into a mixture. It is a powerful antiseptic and obtundent. For hypersensitive dentine take a small crystal and place it in the dried cavity, then take a heated oval or flattened burnisher and touch the crystal with it, which will instantly volatilize part of its mass, going off into vapour, while the remainder disappears into the dentinal surface. It is also useful when a devitalized pulp is sensitive on removal. It can be applied mixed with Dentalone or 5 to Io per cent. in glycerine. In the latter form it is useful in painful apical pericementitis when treating a root canal in the proportion of 2 to 5 per cent.

Erythrophleine Sulphate has been used recently, and is efficacious as an obtundent of sensitive dentine. It must be used cautiously, however, and applied in minute doses of $\frac{1}{40}$ to $\frac{1}{24}$ grain. It is applied in a similar way to arsenic, sealed in the cavity, and left for twenty-four but not longer than fortyeight hours or the tooth may become very sore from slight inflammation of the pericementum. It is a drug that undoubtedly has valuable obtundent properties, but requires some experience in order to get the best results. "Throphleol" is a proprietary preparation containing 50 per cent. of erythrophleine sulphate in Eugenol, which is a convenient form for dental use. (Martindale and Westcott's Extra Pharmacopœia.)

Arsenic, or arsenious acid, or arsenic trioxide, is used as a 
devitalizing agent for treatment of pulps. It is mixed with creosote or carbolic acid as a rule, but other drugs are mixed with it, such as cocaine and morphia to prevent pain in its action (see chapter on Root Treatment). It is also prepared for use as fibre, or pellets of wool and discs to facilitate application. The dose is from $\frac{1}{60}$ to $\frac{1}{10}$ grain. Caution must be used to seal it in the cavity effectually or it may leak out and devitalize gum tissue and bone, also causing necrosis. If it leaks from the tooth at either end severe pericementitis will supervene.

Calcined Alum is a powerful astringent and tanning agent. It is a very useful chemical substance, and forms an important ingredient in mummifying pastes. It is a powerful astringent for controlling hæmorrhage used in conjunction with carbolized resin (see chapter on Extractions).

Thymol is in the form of crystals derived from thyme and other oils. It melts at a low temperature. It is almost insoluble in water I in I,500 parts, glycerine I in 200 parts, equal parts of alcohol and glycerine $I$ in 8 . It liquefies when put with phenol, menthol, and camphor crystals. It is a powerful germicide and very persistent and penetrating. Dr. Miller of Berlin recommended it in combination with other medicaments for use in nummifying pulps. It is very irritating, but its irritant action is modified considerably by liquefying it with carbolic and camphor crystals; it can be used for dressing cavities in live teeth in this form. These combinations are suitable for root treatment in putrescent roots. Dr. Kirk recommends thymol and carbolic crystals for root treatment; it is bland and non-irritating, and he has named it "Thymo-phen."

Thymol crystals well pulverized to a powder are universally used as an ingredient in pulp mummifying pastes. Dr. Soderberg's formula, which has been proved to answer this purpose well, consists of:

Powder $\left\{\begin{array}{l}\text { Pure zinc oxide } \\ \text { Thymol crystals } \\ \text { Calcined alum }\end{array}\right\}$ Equal parts by weight, well pulverized.

Liquid: Pure glycerine. Mix to a paste as required.

Thymol is also largely used as an ingredient of tooth pastes and mouth-washes.

Liquor Formaldehyde or Formalin... This is an aqueous solution containing formic aldehyde gas to the extent of 35 to 40 per cent. It is an active and powerful germicide, and is compara- 
tively non-poisonous. Its use is restricted in dentistry, owing to its intensely irritating properties, but is a potent agent for preventing decomposition and fermentation. It is also used for embalming and mummifying animal tissues for trade and scientific purposes. It may be added to Dr. Soderberg's mummifying powder to the strength of 3 to 5 per cent. by weight and well mixed in order to distribute it equally. If applied by mistake to a cavity in a live tooth it will cause unbearable pain. If used in too great strength in root treatment pastes it may not give rise to much pain but the periapical tissues will be irritated in course of time and inflammation will probably supervene.

Glycerine is an emollient and useful as an adjuvant to prevent irritation from the action of drugs such as carbolic acid, thymol, cinnamon oil, etc. It is also a useful vehicle for securing the penetration of these drugs into the tooth tissues in root treatment owing to its hygroscopic property. Care should be taken to see that it is kept in a specially well-corked or stoppered bottle, as it quickly absorbs water and its properties will deteriorate. Specific gravity I250 is the best strength for use in root treatment. "Glyco-Thymoline" is an excellent mouth-wash for general inflamed conditions of the mouth and throat.

Chinosol is a powerful antiseptic and is used for syringing septic sockets after extractions in the strength of $\frac{1}{10}$ per cent. It is supplied in tabloids of a lemon-yellow colour.

Zinc Oxide is used as an ingredient of mummifying and rootfilling pastes. It is also the base in the manufacture of zinc cements.

Tincture of Iodine is prepared in two strengths. The stronger, called " tincture iodi fort.," or " liniment of iodine," is the most suitable for dental purposes. It is used as a counter-irritant in pericementitis, and when mixed with tincture of aconite is a very comforting medicament for this purpose.

Tincture of iodine is a suitable antiseptic for painting on the gums in order to sterilize the field for injection of a local anæsthetic. It has a decided action on mucous plaques. This property may be applied to advantage when cleaning and polishing children's teeth in cases of green stain where the faces of the teeth have deep, dark streaks which appear to be in the substance of the enamel. The iodine is painted on the teeth with a piece of amadou and allowed to stay for a couple of 
minutes. when the stains and anything else present are lifted out from the surface and easily polished off with tooth-paste and superfine pumice on a polishing brush in the dental engine.

Allowance must be made for the alcohol used in the tincture evaporating, and a little should be added now and again to make up the deficiency. Some operators prefer glycerine of iodine-glycerine 50 parts, iodine I part.

Tincture of iodine is used also as a counter-irritant.

Tincture of Aconite.-This medicament is used in conjunction with liniment of iodine:

$\begin{array}{llllll}\text { Liniment of iodine } & \ldots & \ldots & \ldots & \ldots & 2 \text { parts. } \\ \text { Tincture of aconite } & \ldots & \ldots & \ldots & \ldots & \text { I part. }\end{array}$

It is employed as a counter-irritant and anodyne in periodontitis, painted on the gums around the affected tooth. The dose of the tincture of aconite when used in medicine is 2 to 5 minims. It is a powerful heart depressant, and must be used with great caution. One to 2 minims of the above mixture can safely be applied to the gums, even in heart patients. The best way to control the amount is to use a piece of amadou about $\frac{1}{8}$ to $\frac{1}{4}$ inch square.

Cresylic Acid is an important chemical. It is a mixture of ortho-, meta-, and para-cresols. It is a very powerful germicide, but not very soluble in water considering its strength. Soluble $I$ in 50 of water. It forms the base of a number of well-known germicides on the market. Lysol is a saponified cresol, very useful for sterilizing instruments, as they do not rust when kept for some time in a weak solution. Strong solutions will attack the electroplating. It is a reliable sterilizing agent and germicide. A 2 per cent. solution ( 2 teaspoonfuls in I pint of water makes a 2 per cent. solution) is sufficient for instruments. Blood should be washed off first in cold water.

Camphenol is a cresol compound solution, manufactured by Messrs. Johnson and Johnson, New Brunswick. It is composed of cresol, camphor oil, and gelatin, and is admirably suited for use as an antiseptic and healing mouth-wash in septic and inflammatory conditions. It has a distinctly healing action on the oral tissues, and has proved invaluable for relieving pain in pericementitis, dental abscess, and pyorrhœa. If the patient is in great pain, a $\frac{1}{2}$ per cent. solution used hot every half-hour or hour will invariably give relief. The patient can make a I per cent. solution as a stock bottle, pour out as required, adding an equal quantity of boiling water, which reduces it to 
$\frac{1}{2}$ per cent. and is hot. It is also very efficacious in sequelæ cases after extraction of teeth; it stimulates healing in a marked manner. The writer has carried out investigations with this preparation, and finds it invaluable for preventing trouble and also for dealing with it when it occurs. It is supplied in I-ounce bottles, and can be given to the patients with instructions written out for their use.

Sulphuric Acid is used as recommended by Dr. Callahan for placing in the pulp chamber of a dead tooth, such as a molar, in order to find very fine and obscure canals. He recommends a 50 per cent. solution for this purpose. A tiny pellet of asbestos wool saturated with it is placed in the cavity and sealed in for twenty-four hours, when a black spot will show where the canal is. It is also useful applied with a wisp of asbestos wool, or a platinum broach, to deal with sensitive remnants at the end of a root canal after devitalization with pressure anæsthesia.

Zinc Chloride is a caustic and anti-putrescent. It is very poisonous. Is very useful for stimulating indolent sockets that show little inclination to heal some time after the extraction of teeth. Syringe the socket or dry it out, then introduce a tiny piece of crystal or pass a silver or platinum ball-ended probe with a little zinc chloride on it. It is very hygroscopic, and there is usually some liquid in the bottle. On account of its stimulating properties on septic tissues zinc chloride aural drops (guttæ zinci chloridi [aural]) is a useful form for pyorrhœa pockets:

\begin{tabular}{|c|c|c|c|c|}
\hline Zinc chloride & . & $\ldots$ & - & . \\
\hline Glycerine & $\cdots$ & & & \\
\hline Alcohol .. & .. & & & . \\
\hline
\end{tabular}

Must be used with caution (Martindale and Westcott).

Copper Sulphate is a very poisonous chemical, but is a potent germicide and astringent. Dose, $\frac{1}{5}$ grain. It is used in pyorrhœa by powdering the crystals and packing them down into the pockets, but caution is necessary as to the amount used, and too large an area must not be attempted. It is soluble in water I in $3 \frac{1}{2}$, glycerine $I$ in 2 , insoluble in alcohol.

Bifluoride of Ammonia.-This preparation has been investigated by Dr. Joseph Head, M.D., D.D.S., Philadelphia, U.S.A., as a specific for pyorrhœea and a solvent of tartar deposits on the roots of the teeth. It is well worthy of attention (see Dental Cosmos, IgI2, p. 4I). 
Aromatic Sulphuric Acid.-The United States Pharmacopœia preparation is very useful for hyperæmic gums that occur around the necks of teeth and where crowns are present. It has been used as a caustic, styptic, and antiseptic.

Trichloracetic Acid.-This is a chemical substance prepared either by the chlorination of acetic acid or by the action of acetic acid on chloral hydrate. It is in the form of deliquescent crystals, melting at $55^{\circ}$, very soluble in water, alcohol, and ether. It is a quickly acting escharotic. The application of a $5^{\circ}$ per cent. solution produces a dry adhering eschar which is quickly thrown off. There is said to be no secondary inflammation. For use as an astringent lotion or gargle $I$ to 5 per cent. in water may be used, or I in I of glycerine for local application. Tobacco stains on the teeth can be removed by its cautious use, 25 per cent. or less. In the early stages of pyorrhœa, after swabbing out the pockets with Io or 20 volumes strength peroxide of hydrogen, it is a practical remedy and well worth trying as it has a powerful astringent and drying action as well as being escharotic. It appears to drive the blood out of swollen hypertrophied gum. This property of drying may be tried when superficial bleeding occurs when filling a cavity near the cervical edge or when putting a crown on.

Its action in pyorrhœe pockets is probably dual-killing the infective organisms and dissolving the calcium carbonate of the tartar. It should be tried before sound teeth are sacrificed: (See Martindale and Westcott's Extra Pharmacopœia, "Acida Chloracetica.") The writer has given some attention to this drug and finds that it is well worthy of a prominent place in the dental armamentarium.

Hypochlorites of Calcium and Soda have been brought, during the last few years, into extensive surgical use with remarkable success. They contain hypochlorous acid, which is stated to be the most powerful antiseptic known. "Eusol," Dakin's solutions, chloramine " $T$ " and di-chloramine " $T$ " are well-known preparations which come under this heading.

Phenol Sulphonic Acid is particularly useful where a powerful stimulating antiseptic is needed, such as in fistulous tracts, bone absorption around the apices of teeth as found in chronic abscesses, pyorrhœa pockets, and as a preliminary treatment in the removal of calcareous deposits about the teeth. The detachment of bone sequestra in necrosis is enhanced by its 
early application. It may be used as a substitute for Dr. Callahan's 50 per cent. sulphuric acid for the enlargement of root canals. It is applied in pure form or 50 per cent. solution with an iridio-platinum loop or a broach of the same metal wrapped with fibres of asbestos wool (see article in Dr. Prinz's Materia Medica).

Martindale mentions a 3 per cent. solution as being useful in gingivitis and pyorrhœa. It reduces swelling, arrests flow of pus, and the gums return to their natural shape. As this medicament is such a useful remedy in septic cases we will quote Dr. Buckley's description of it in full from the Dental Cosmos, Igro, p. 434:

"In complicated abscesses, good results may be obtained by forcing phenol-sulphonic acid in contact with the bone or forcing it through the tooth and sinus, adding as much water as you have of both constituents, and thus using it in a $5^{\circ}$ per cent. strength.

"There is a little trick of pharmacy associated with this remedy. If water is added to phenol sulphonic acid, it will precipitate the phenol. If, however, the phenol is heated, and while it is hot an equal amount of sulphuric acid is gradually added, and then, while the mixture is still hot, an amount of water equal to the mixture, phenol-sulphonic acid $5^{\mathrm{c}}$ per cent. strength is obtained, which can be diluted to any strength and used wherever indicated. Sometimes this solution is applied to pyorrhœa pockets. I do not like to use acids in treating pyorrhœa because a hypersensitiveness of the teeth is produced by their use. It is seldom necessary to use acids in treating pyorrhœa, but sometimes the pus persists in forming in a pocket even after the roughened portion of the bone and all of the destroyed pericemental membrane seems to have been thoroughly removed mechanically or surgically. This flow of pus is sometimes stopped by injecting (or swabbing) phenol sulphonic. acid into the pocket. In that case a 15 per cent. solution suffices, but unless the precaution of heating the remedy in making it has been taken, the solution cannot be diluted without precipitating the phenol."

Menthol occurs as needle-shaped crystals, which are deposited on cooling peppermint oil. It is used for various purposes, but is somewhat refractory for use in solutions. It is practically insoluble in water, insoluble in glycerine, soluble 5 in $\mathrm{I}$ of absolute alcohol. 
Creosote is a mixture of phenols, guaiacol, and cresol. It has an unpleasant, persistent odour. It was widely used many years ago, but its use has been largely replaced by more suitable preparations. If employed at all it must be beechwood creosote, and should not be confounded with coal-tar creosote, which is very poisonous. It is still used, however, by many operators with much success as a medicament for root treatment. It is one of the most powerful deodorizers, anti-putrescents, and antiseptics. Being a caustic it must not be used in full strength. For root treatment mix I drachm of beechwood creosote with 7 drachms of glycerine, specific gravity I250 (see chapter on Root Treatment and Filling).

Nitrate of Silver is a powerful caustic and astringent. It is used in dentistry as an application for arresting dental caries and erosions on the surface of the teeth. It is very useful for this purpose as these surfaces are usually very painful for shaping into cavities for filling. It is also a powerful coagulator of albumen, deeply penetrating and rapid in its action. It turns everything it touches black. It is very poisonous; the average dose is $\frac{1}{5}$ grain. The compound formed by the nitrate with the organic constituents of the tooth is insoluble, with the exception of the presence of a few substances. The nitrate can also be absorbed by the affected dentine and stimulates the subjacent dentine to more healthy action. (See article on Silver Nitrate in Dr. Prinz's "Dental Materia Medica and Therapeutics").

Dr. Howe's method of root treatment with silver nitrate is well worthy of attention.

Growths in the mouth, such as small tumours, polypi, or hypertrophied gum, are readily destroyed by applying the crystal or a concentrated solution. As it is so poisonous it must be used with great caution. The safest way of applying it is by taking a silver wire, heating it, and plunging it on to a crystal, forming a bead, or if a solution is used, take a tiny square of amadou held in the tweezers. The stains of silver nitrate are removed by a solution of sodium hyposulphite or ammonium chloride.

We have devoted a chapter to the consideration of drugs used in dental surgery, in order that our readers may become conversant with their nature. Practitioners are apt to regard drugs as being incidental to the various methods of operating, whereas they are factors in themselves and well worthy of 
consideration and investigation as to their merits, potency, and action. It is only by personal observation, therapeutically, that one can know them authoritatively.

Dr. Buckley has said: "It is strange how some practitioners expect to find one drug or remedy which can be used in every case all through their practice."

We have pointed out that the employment of drugs for curative or remedial purposes depends on the conception of two subjects which are intimately bound up with each other, Materia Medica and Therapeutics. To put it in another way, it consists of the drug and the organism. The practitioner has at his command an armamentarium which is quite as potent and reliable as the instruments which he uses. It remains, therefore, to learn how to use and manipulate it in order to fully utilize. the whole of his equipment.

REFERENCES.

Dr. Prinz's " Dental Materia Medica and Therapeutics."

Dr. Buckley's " Dental Materia Medica."

Martindale and Westcott's Extra Pharmacopœia. 


\section{CHAPTER XIV}

\section{LOCAL ANESTHESIA OR ANALGESIA}

THE use of local anæsthesia is considered a great boon both to the patient and the dental operator, as it can be employed at any time with ready convenience. In general surgery it has been brought to a high scientific level with methods of block anæsthesia for the facial regions and upper limbs; also spinal analgesia for operative measures on the abdomen and lower limbs. In spinal analgesia an injection of a local anæsthetic is made in the lumbar region under supreme aseptic conditions.' The cerebro-spinal fluid which occupies the spinal canal is drained off and the anæsthetic is again injected with a large quantity of a weak solution. The cerebro-spinal fluid is constantly being secreted, and when the anæsthetic has been absorbed, the canal becomes normal. The certainty of anæsthesia is a constant factor and precludes any doubt as to the patient being completely insensitive to pain. The cerebro-spinal nerves running off from each side of the spinal cord are affected as the anæsthetic solution rises, until the surgeon is satisfied that it has reached the region he desires. If continued it rises until it reaches the ventricles of the brain and universal analgesia supervenes, which in some cases extends to the mouth and the nose.

Block anæsthesia secures a similar guarantee of painlessness. by the anæsthetization of the nerve-trunk which controls the zone of operation.

In dental operating the production of local anæsthesia is quite an insignificant matter compared with block and spinal analgesia, yet we are faced with conditions that are not so scientifically definite. We are called upon to extract a tooth in the routine of a busy practice. The operation must be done quickly. There is always present the uncertainty of the extraction of a tooth being entirely painless. Fortunately the majority of cases are painless. The point which we wish to emphasize is that the scientific accuracy accompanying major operations, 
anatomically and physiologically renders such procedures an exact science.

For dental operating the profession has universally decided, so far, that the analgesic effect shall take place in the direct region of the tooth structures. For the extraction of teeth the anæsthetic solution has to be injected into the gums, and reliance has to be placed on the anæsthetic, and great pressure, in order to reach the structures beneath the bone. The nerve filaments do not come into direct contact with the anæsthetic; it has to reach them rather than their being the centre of the anæsthetic zone (see Fig. 45). The success of this method is dependent, therefore, on the skill of the operator to take full advantage of the little things which are present to get the anæsthetic to the periodontal membrane which surrounds the root of the tooth to be operated upon either for extraction or cavity preparation.

The drugs employed for local anæsthesia in dentistry are usually cocaine hydrochloride, beta-eucaine hydrochloride, and novocain.

Cocaine is an alkaloid of the coca plant. An alkaloid is the active principle of a drug. Alkaloids occur in many plants and some in animal tissues. They form salts with acids, such as hydrochlorides, lactates, nitrates, etc. They are generally crystalline bodies, and act powerfully on the animal system, and are used in medicine as morphine, strychnine, etc. They are usually violent poisons.

Cocaine as a local anæsthetic has been in use for many years, and went through an experimental period when practitioners naturally took full advantage of its powerful anæsthetic property. But it was found also, in the large doses employed, that it had alarming systemic effects, and several deaths were reported. However, its use is now better understood, and in dentistry it may be employed in $\frac{1}{2}$ to $\mathrm{I}$ per cent. solutions with comparative safety if suitable judgment and precautions are taken. There is no doubt that the purity of the alkaloid is the first consideration. There are two varieties of plants that produce the coca drug: one is grown in Northern Peru, and the other in Bolivia, which is considered better for medicinal purposes. The preparation of the alkaloid by reliable manufacturers is another factor.

The unfavourable symptoms which gave cocaine a setback through its incautious use gave chemists an impetus to investigate the subject, which resulted in eucaine and novocain being pro- 


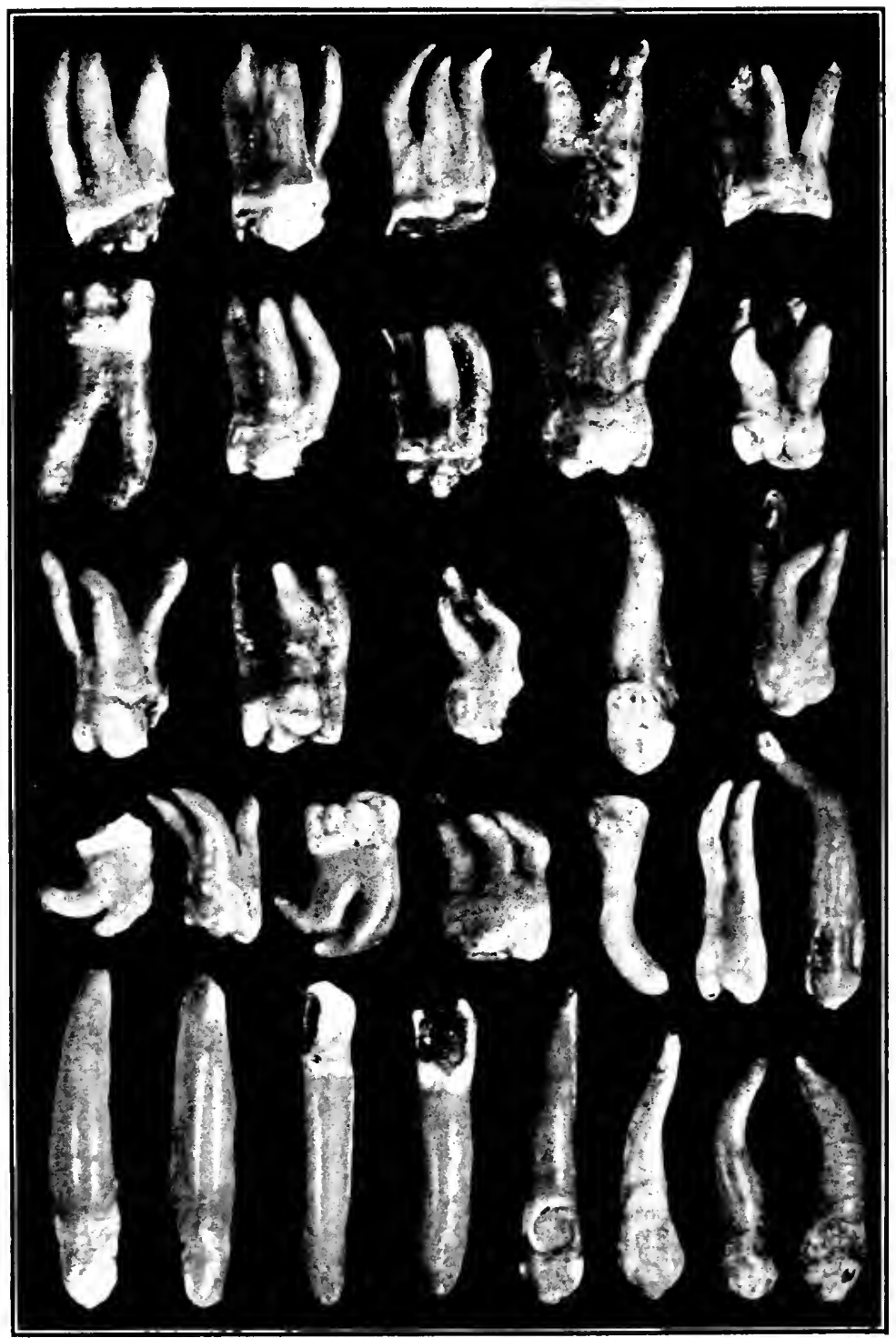

Fig. +3.-Difficllt Extractions by Various Operators with Local ANESTHESIA. 
duced as synthetic alkaloids for substitution of cocaine. Novocain has given great satisfaction on account of its efficiency and greater safety; authorities agree that it is six or seven times less toxic than cocaine.

Cocaine, however, still has its advocates. The writer has had opportunities of forming an opinion that it has a very wide use in spite of those who find novocain safer. One half to $\frac{3}{4}$ per cent. of cocaine is used as a safe solution. The dose of the alkaloid is $\frac{1}{4}$ grain. One grain dissolved in roo minims of water is $x$ per cent., so that $\frac{1}{4}$ grain equals 25 minims of $I$ per cent. solution or 50 minims of $\frac{1}{2}$ per cent. solution. Chloretone added to the solution is claimed to preserve cocaine solution and to enhance its anæsthetic effect. It is soluble I part in 200 of water.

Eucaine is not largely used in dentistry.

Adrenalinum or suprarenin is added to the anæsthetic solution in order to localize its action to the region of the tooth to be extracted and to retard its absorption. When this drug is present the solution should be colourless and clear; if decomposition has set in it will become pink, and finally brown; in this condition it must be discarded. Many operators realize the potency of this drug, and it is well to understand why we use it, the dose we are administering, and the action it is producing on the tissues. It is a powerful vaso-constrictor, and the strength usually employed with cocaine is $I$ in 50,000 , but many men prefer $I$ in roo, ooo because it is desirable to curtail the period of its action. The reason for this is, that if vaso-constriction continues, which causes a condition of stasis or arrested circulation, the resistance of the blood cannot counteract septic development if infection is present.

Novocain is a vaso-dilator and therefore requires a larger proportion of adrenalin or suprarenin. Dental tablets $\mathrm{E}$ are supplied containing:

Novocain, 0.02 gramme, or approximately $\frac{1}{3}$ grain.

Adrenalin, o.0005 gramme, or about $\frac{1}{1000}$ grain.

One tablet is dissolved in I cubic centimetre of water or approximately $x 7$ minims, which makes the proportion about $\mathrm{I}$ in $\mathrm{I} 7,000$. The same percentage of novocain as tablet $E$ is supplied in solution already prepared, and contains in addition, thymol $\frac{1}{100}$ grain, approximately. The addition of thymol to novocain (and also to cocaine) solution has been advocated as a useful and non- 
toxic antiseptic, and assists the anæsthetic action. The strength of $I$ in I,700 ensures its complete solution, as the solubility of thymol is $I$ in $r, 500$. If the thymol could be added to the dental tablet $\mathrm{E}$ it would be appreciated by many operators. Some men prefer to lessen the proportion of adrenalin; this can be done by putting two tablets dental $\mathrm{E}$ with one tablet $\mathrm{F}$ (without adrenalin) into 75 minims of sterilized water, which makes a 2 per cent. solution or thereabout. The strength of adrenalin would then be about $I$ in 3,400. Another important factor in the anæsthetic solution, whether it is cocaine or novocain, is that it should be rendered isotonic by the addition of sodium chloride, $I 5 \frac{3}{4}$ grains to 4 ounces of sterile water.

Dr. Halliburton has described the importance of Ringer's solution and the investigation of its constituents in the blood, and also the cerebro-spinal fluid. Fischer's modification of Ringer's solution is considered an improvement for dental purposes and is supplied in tablets for preparing the solution for novocain. It consists of the chlorides of sodium, potassium, and calcium.

The object of using physiological or normal saline solution, or Ringer's solution, is to render it isotonic. In this condition it is non-irritant to the cells of the tissues. The anæsthetic solution is carried into the cells by a principle called " osmosis." This process is well understood by physiologists and chemists. It consists of the passing of dissimilar chemical substances and gases through a membranous diaphragm or membrane. There are many instances of it in the animal body, and also plants, whereby their functions are carried on.

If the anæsthetic solution is of less density than the blood it will cause swelling of the cells and is said to be hypotonic; if of greater density it will cause shrinkage and is said to be hypertonic. If it is isotonic, it is of the same specific gravity, or freezing-point, as the blood, and will not irritate or disturb the normal condition of the tissues, whereas hypotonic and hypertonic solutions will cause disturbance and probably sloughing or gangrene.

The hypodermic instruments include a good syringe for the injection, suitable hypodermic needles, a Dappen's glass for holding the solution when in use, a test-tube for bringing the solution to the boiling-point, especially with novocain, a holder for the test-tube, a large glass-stoppered pot for holding the 
syringe and needles when not in use, another one for holding the anæsthetic bottle or tubes. These pots keep dust away and preserve the instruments from air contamination. A wellmade electro-plated metal case will hold the instruments very well.

The main instrument is the hypodermic syringe. This should be strong and withstand considerable handling both in use and in constant cleaning, when it is liable to be dropped. An all metal one answers this purpose better than a glass one. There are glass compounds on the market for scientific purposes, such as fused silica tubes and "Vitreosil" tubes, which would be strong enough. They can be bought with closed ends. Such a tube could be improvised, but as there is no practical advantage in having a clear barrel it is better to avoid having the necessary joints, which after a time get out of order. There are many syringes on the market, the D. M. Co.'s "Washerless Syringe" and the "Imperial" are popular. The former consists of a hard rubber core at the end of the plunger, the latter has washers or packing in the head of the barrel. In spite of the number of syringes which have been put on the market to appease the operator's annoyance, it appears from experience, so far, that packing and washers are necessary. The point to consider, then, is, how can we keep the working parts of the syringe clean and the packing and washers in a desirable condition? There is more in this point than the manufacturers seem to appreciate. Many operators have a box containing syringes that have been discarded, the metal parts are beautifully made, but the joints and washers have rendered them useless. There are two things to be learnt from this discarded box. One is that the metal parts are as good as new in appearance, which proves that the metal keeps in perfect order when kept quite dry and away from the air. The second thing is that where washers and packing have been, the metal is corroded and black. We have discovered soon after use that washers and joints disturb our peace of mind, not from an aseptic point of view, because we have boiled or otherwise sterilized them, and this process only aggravates the trouble, but because of corrosion at these parts. A syringe comes to us well greased, and the first thing to do is to clean it, but how are we to clean leather washers, or any kind of washer, without impairing them ? Particles also break off and clog up the nozzle. Rubber is equally unsatisfactory 
because the corrosion disintegrates it quicker than other packing. Rubber, however, can be boiled and cleaned by itself, which is satisfactory, but it must not be left in contact with metal. Salts of nickel and brass are very poisonous; damp affects them quite apart from being wet. If a syringe has been perfectly cleaned and well dried, but the interior left wet, or even damp, it will produce a blackish stain on clean cotton-wool or a napkin within twenty-four hours, and if washers and packing are present the staining will be greater. Therefore all washers or any part of the syringe where the anæsthetic solution comes into contact, and damp can get in or underneath, should be taken out at least once a week. Some operators go some time, perhaps a month, without requiring the use of a hypodermic syringe. Such is

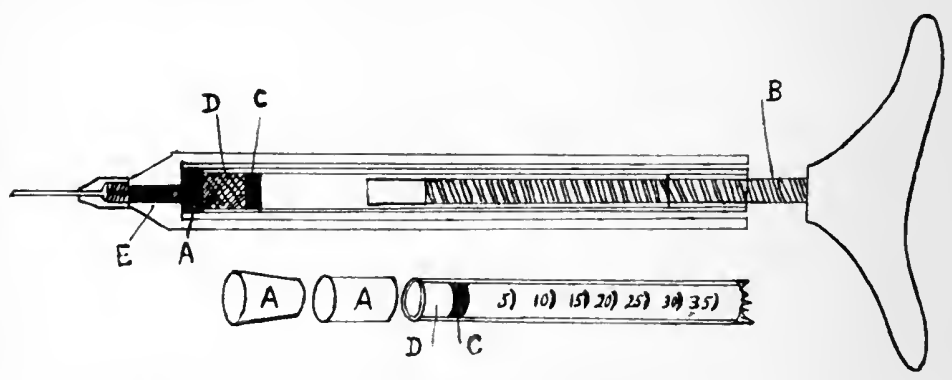

Fig. 44-Author's Suggestion for keeping a Rubber Plug separate from the Metal Piston when not in Use.

A. End of cork fitting in barrel; D, C, body of cork fitting in plunger; $\mathrm{E}$, hole of nozzle leading to needle; $\mathrm{B}$, extending handle bar with screw seating in plunger tube.

often the case in a busy conservative practice. When it is required and the syringe shows evidence of oxidation around the washers he discards it. In a busy practice in an industrial centre where the syringe is in constant daily use it does not matter so much, as it is being constantly cleansed by the solution and the amount of oxidation would not appear so great. However, if we could keep the metal parts separate and adjust the washer at the time of using only it would obviate the trouble.

The writer has improvised a syringe which consists of a strong, open, tubular piston with the usual extending screw bar at one end to hold a rubber cork which must not be too soft. The tube must not be blocked in any way, as the cork plunger will not stay in position. A vela rubber cork can easily be made by forming one accurately in wax, then making a plaster mould 
from it, which gives a matrix in which wax forms can be moulded and removed. In flasking, the cork should be completely covered excepting the end which goes into the piston. A cork can easily be put into a flask when flasking a plate. If they could be supplied by the dental manufacturers so much the better. The hypodermic needles, being made of metal, come under the above statement, so that after use they should be cleansed from blood, well washed and dried by blowing hot air through them, and carefully put away. There is no need to put a wire through them.

Operators vary in their methods of injecting the anæsthetic solution. Some depend on the direction in which the arterial blood is flowing and allow for that accordingly. But in any case our object is to reach the periodontal membrane which lines the alveolus of the tooth to be extracted. As we have already stated, the success of our methods depends on a few important points:

I. Knowledge of the nature of the bone.

2. The nature of the soft tissues involving the gum and bloodvessels.

3. The confidence in our anæsthetic solution and its osmotic properties.

4. The mechanical pressure to be applied with the syringe.

Although we have separated these points in order that they may be appreciated, they become one in the application of the injection.

The examination of the bone of the jaws in detail is recommended. Take sections at various parts.

We shall find in the upper that the teeth lie in the outer portion of the bone, right round from wisdom to wisdom teeth, with the exception of the inner roots of bifurcated bicuspids and the palatine roots of the molars. Therefore it is of no use to inject on the inner or palatal side excepting at the gum margin, which is the only part likely to reach the alveolus and the palatine molar roots.

In the lower, the teeth lie near the front portion of the bone as far as the canines, the bicuspids lie deeper at the apices, the molars lie towards the centre, and are difficult to reach.

There is a great difference between the upper and lower jaws. The upper is more favourable for successful local anæsthesia, as the bone, although more solid generally in the alveolar region, 
is more porous and admits of the transmission of the anæsthetic effect. Moreover, the gum is thicker and will hold the solution better. The lower jaw has a thick outer or cortical plate which is very dense, almost like ivory, but there are tiny perforations about the septa between the necks of the teeth; the gum is thinner than in the upper, and the face of the outer surface is concave so that the needle cannot travel down as it can in the upper. The path of the injection should be down the centre of the axis of the root of the tooth where the bone is necessarily thinnest (see Fig. 45). The use of a straight needle is all that is usually necessary, and if we realize the direction that the anæsthetic should travel we shall have success. The gums vary in their nature, some tissues are thin and anæmic, others

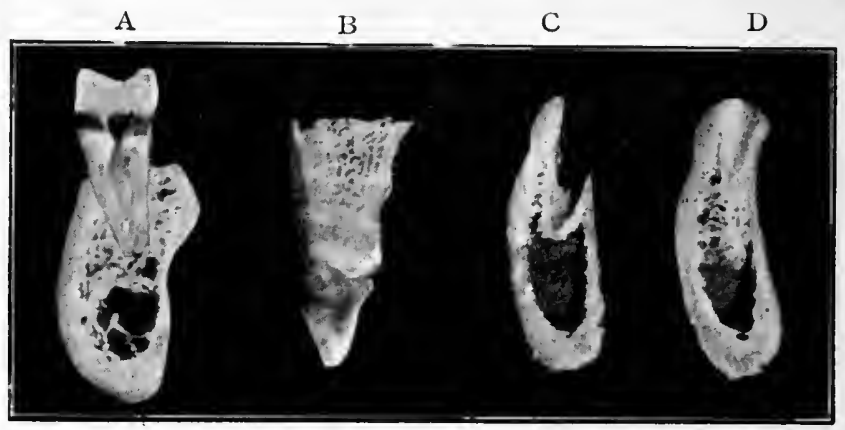

Fig. 45.-Sections of Bone.

A, Lower twelve-year molar; B, upper canine; C, lower second bicuspid, centre of socket; D, lower second bicuspid, side of socket.

are thicker and flocculent in appearance, and some are swollen and congested. If an abscess is indicated either by swelling or a fistulous opening it should be avoided and the injection made around it. It requires the practice which comes from experience to effect the entrance of the needle in the varying conditions of the gums. If the gums are healthy and dense the anæsthesia will probably be better, as the pressure will carry the solution well in. In loose tissues difficulty will be found to retain it. We must observe the face of the gum and avoid superficial veins, which can be seen. If the needle is carried up the face of the gum very far, care must be taken to tighten the soft tissues which form the junction of the lips and cheeks, as the solution will quickly disappear and be useless, whereas we want it directly against the bone. Care also must be taken not to 
push the needle against the bone or the point of the needle may break. The rustless steel needles supplied by Messrs. De Trey and Co. are excellent, they are fine in size and easily penetrate the gum, and in the lower are more likely to conduct the solution without leakage. Copper-headed needles are also good, because they do not jamb in the nozzle. It is only necessary to well inject on the labial and buccal surface of the gums, starting at a point about an eighth to a quarter of an inch from the gum margin, which allows the solution to travel round the gum about the neck of the tooth to reach the periodontal membrane, which will then conduct it down the alveolus. In the upper the bone is usually thin at the apical part of the roots, and advantage can be taken of this. The tongue side of the mandible and also of the palate is near fairly large bloodvessels. We must. observe the positions of these. This risk can be avoided by using the external side only. In the palate there are the anterior and posterior palatine canals and also the palatine blood-supply which ramifies the palatal surface. On the external surface of the lower we should remember the position of the mental foramen.

The precaution must be taken of expelling any air that may be in the syringe before injecting, as a bubble of air which might possibly get into a bloodvessel, especially a vein, might cause serious trouble, and if it reached the heart would probably cause alarming syncope. The best way is to tap the syringe in an upsidedown position and then squirt a little solution out of the syringe.

Pressure in injecting must be applied according to the density of the tissues. Undue pressure sometimes results in a white zone on the face in the region of the infra-orbital foramen in the upper, and the same phenomena in the lower, in the region of the mental foramen, and if it is very intense will anæsthetize the corner of the lips.

Many practitioners make a practice of giving every patient, when using cocaine, a dose of stimulant to act as a carminative. Validol is an excellent one, tincture of strophanthus with cardamoms is a powerful one (see end of chapter on Stimulants). If a patient is very nervous, or of a weak type, it is wise to administer a stimulant such as Messrs. Parke, Davis and Co.'s Nitroglycerine Elixir, Black's Cardiactis, or Bowen's Anti-shock.

The area of the gum for injection must be rendered aseptic before attempting to place the needle into it. Tincture of iodine or Dentalone are useful for this purpose, particularly the 
former. If the mouth is not a healthy one the patient should be advised to use a mouth-wash, night and morning, of peroxide of hydrogen, or Camphenol, three or four times a day, for a day or so. Peroxide of hydrogen ought not to be used at the time of injection because of the danger of driving tiny bubbles of gas into the tissues.

Some patients will exhibit signs of faintness and even collapse, partly under psychological imagination, the physiological action of the solution on the nervous and circulatory system, or from shock. Fortunately these cases are not frequent, but the operator must be prepared for them.

The symptoms of cocaine poisoning are not often seen now that the methods of injection are better understood. Large round beads of perspiration, of a characteristic type, are seen on the forehead, the patient feels dizzy and faint, with rapid and feeble pulse, difficult breathing, lips blue, and pallor. If the symptoms are not attended to they will become alarming, with respiratory spasms, and syncope following.

The first treatment is to administer a quickly acting stimulant such as aromatic spirit of ammonia, dose, 30 to 60 minims in water. Tincture of strophanthus is useful but very slow in action, dose, 2 to 5 minims in water. As this is such a powerful agent it is better mixed in a quantity, and the use of fresh proprietary restorative compounds obviates the difficulty. A second dose should not be given under half an hour afterwards. Push the patient's head well down between the knees. Postural treatment is very effective by placing the patient in a horizontal position with the head lower than the feet in order that the blood will gravitate to the head and reach the medulla (see Chapter IX.). If the symptoms become severe inject $\frac{1}{60}$ grain of strychnine sulphate; this is supplied in convenient capsules or ampoules for the purpose. Amyl nitrite is useful in threatened syncope. It is a powerful vaso-dilator, administered usually by inhalation: dose 2 to 5 minims. It is supplied in glass capsules covered with silk, which are crushed between the fingers to release the vapour; 3-minim capsules are convenient (Martindale's). It is largely employed as a restorative in bad cases of fainting.

Death through overdose of cocaine is by asphyxia, therefore attention must be given to the breathing, and if necessary artificial respiration must be performed. Inhalation of oxygen is useful. 
It is not prudent to attempt injection in a very septic mouth or if there is a severe abscess, especially in the lower jaw in the wisdom tooth region. There is extreme danger of severe secondary infection occurring both from the vaso-constricting action of the solution and the pressure from the syringe driving pus into the deeper tissues. The administration of nitrous oxide gas is suitable for such cases. If this is not possible such cases could be dealt with by the application of a topical solution of an anodyne. The following method, although an old one, is well worth consideration. It has constantly been used by the writer for twenty years with success for the extraction of teeth in young children, elderly people, and heart patients, and for the septic lower molars already described.

The employment of the method depends entirely on the gum being thoroughly dried. This is done with large pieces of amadou. The neck of the tooth and well around the whole region of the roots is painted with I or 2 minims of:

$\begin{array}{llllll}\text { Liniment of iodine } & . . & \ldots & \ldots & \ldots & 2 \text { parts. } \\ \text { Tincture of aconite } & . . & \ldots & \ldots & \ldots & \text { I part. }\end{array}$

This is applied with a small piece of amadou about $\frac{1}{8}$ or $\frac{1}{4}$ inch square. It is then followed by painting, in the same way, 2 or 3 minims of the following mixture:

$\begin{array}{llllll}\text { Liniment of capsicum } & \ldots & . . & . . & 3 \text { drachms. } \\ \text { Oil of cloves .. } & . & . & . . & . . & 20 \text { minims. } \\ \text { Oil of peppermint } & . & . & . & . . & 20 \text { } \\ \text { Absolute alcohol } & . & . & . & \ldots & 2 \text { drachms. }\end{array}$

The patients are directed to keep their mouth open and not to swallow. When they wish to do so they are to rinse their mouth out with warm water, once only. In the meantime we prepare a hot mouth-wash in a tumbler. Bobœuf's Phénol Sodique, which contains some oil of tar, is an excellent preparation for syringing painful septic sockets where severe pain is caused by the inflamed conditions. If used quite hot and discharged well into the socket with a fairly large syringe, immediately after the extraction it gives almost instant relief. The suitable strength for this purpose is a teaspoonful in a third of a tumbler of hot water or less water if it is very painful. This is not a suitable mouth-wash for syringing if cocaine or novocain has been injected, because there is alkali in it. An alkali will precipitate an alkaloid. The best mouth-wash for such cases is carbolic acid I in I 20 to 200 parts, or $\frac{1}{2}$ per cent. Camphenol. 


\section{CHAPTER XV}

\section{THE ADMINISTRATION OF NITROUS OXIDE GAS ALONE, OR IN COMBINATION WITH AIR OR OXYGEN, FOR DENTAL OPERATIONS}

Nitrous oxide gas has been used in dentistry for the last sixty years or more. It has gone through a strenuous investigation by many eminent men. Although it went through periods of popularity and for a time was in rivalry with local anæsthesia, yet it has never been abandoned or condemned, but has held a firm grip in dental surgery for short operations. The shortness of the available anæsthesia was the main drawback or limitation to its use. It was a matter of controversy as to whether nitrous oxide was really a true anæsthetic. Many medical men contended that it produced anæsthesia by partial asphyxiation, and was therefore a dangerous agent. This view was favoured by them because, being well versed in the administration of the hcavier anæsthetic vapours, ether, chloroform, and alcohol, they were apt to attach too much significance to the asphyxial symptoms which with the heavier anæsthetics are alarming in their importance because they have the patient soaked with a drug, whereas with nitrous oxide its effects are purely gaseous and are eliminated quickly. The outcome of many years of constant investigation and practical experience in thousands of gas cases by such men as Sir Frederick Hewitt of London, and Dr. Charles K. Teter of Ohio, has placed nitrous oxide gas in a highly creditable and safe position as a true anæsthetic.

Dr. Teter states that the dangers of nitrous oxide are technical only, while the dangers from all other anæsthetic agents are pathological as well as technical.

Sir F. Hewitt, in his work on " Nitrous Oxide and Oxygen for Dental Operations," has demonstrated beyond question that nitrous oxide gas, $\mathrm{N}_{2} \mathrm{O}$, is perfectly safe, and that the dangers which arise from asphyxial phenomena are caused by deprivation of oxygen or by mechanical obstruction.

Nitrous oxide gas is the anæsthetic par excellence for dental 
work, and its use has been well extended into general surgery for many major operations under certain conditions. It is also being used for producing analgesia for preparing cavities, light anæsthesia for nerve canal work and extracting pulps.

Operating on a patient under its influence demands a different style of operating to that of local anæsthesia because the patient is unable to help himself or the operator, who has to see that his work does not interfere with the patient's respiratory functions. Also he has to be more alert and agile with his hands in order to lessen the time and spare the patient unnecessary anæsthetic fatigue.

There are, and have been through all these years, many men who are expert at gas operations and who hold the anæsthetic in high esteem because they know by long experience that if skilfully administered it guarantees a painless operation. Of course, there are cases which are exceptional, but they are very few, and with the latest improvements in gas apparatus these exceptions are very robust, strongly built patients of both sexes whose thoracic capacity syphons more air into the lungs than the anæsthetic can overcome. But even with this type of patient it does not necessarily mean that they will not be suitable for successful anæsthetization. Alcoholic patients, of course, give some trouble, and it is a question whether abstaining for a day makes matters much better.

Now there are two definite methods of administration with nitrous oxide gas:

r. Short method, with the use of the ordinary face-piece.

2. Prolonged method, with the use of a nasal inhaler.

In general surgery, when long operations are performed, the prolonged method is conducted with a face-piece which entirely precludes the possibility of air getting in, and the patient can breathe either through the nose or mouth.

The first method has been in use since gas was first used in dentistry, although the face-piece is less cumbersome and engulfing in appearance than those in use years ago. We must be careful with certain types of patients or those who have certain diseases or probable lesions from them.

The following conditions require special care in order to avoid continued or well-marked asphyxial symptoms:

Endocarditis, or inflammation of the endocardium which is 
the internal serous membrane of the valves and cavities of the heart, usually caused by rheumatism and accompanied by well-marked valvular murmurs.

Bright's disease, with albuminuria, especially after scarlet fever.

Arterio-sclerosis, a brittle condition of the arteries, which fail to accommodate the pulsation caused by the heart's beat. This condition throws a strain on the heart, which would be seriously embarrassed if asphyxial strain were added under gas.

Valvular disease of the heart caused by rheumatic and other fevers.

Aortic aneurism, which is an overstrained condition of the aorta often caused by a localized arterio-sclerosis. It is in the nature of a tumour caused by the weakening of the arterial wall.

Goitre, an enlargement of the thyroid gland, will swell under the influence of gas, causing a restriction or constriction of the larynx. Exophthalmic goitre consists of enlargement of the thyroid gland accompanied by a prominent development of the eyeballs and an increased action of the heart.

Anæmia or bloodlessness, which is fairly common in young women.

Pregnancy.

Chronic alcoholics.

Heavy tobacco smokers.

These cases also are contra-indicated with cocaine.

Assuming that the patient is fairly healthy and can come into the surgery looking fit, there is no danger from $\mathrm{N}_{2} \mathrm{O}$ gas when administered by an experienced man, but care, of course, is needed.

The apparatus and instruments for the administration of nitrous oxide gas consist of:

Gas stand; gas bottles 25-, 50-, 100-, or 200-gallons capacity; 2 to 5 gallon rubber bag; tubing from bottles to bag and from bag to face-piece; a three-way stopcock to control gas and air; and a face-piece. An assortment of gags, plain vulcanite and Hewitt's are best for ordinary cases, Brunton's gag, Buck's gag, or any special gag that may be required. The simpler the gag is in construction the better. No rubber pads are required either on mouth-gags or Mason's gag; they are apt to slip when blood is about and they are uncleanly. A wedge for prising the mouth open, or, failing that, the handle of a pair of forceps will answer the purpose. Mason's gag, tongue forceps, (there are various patterns, but the smaller the blades the 
better), sponge holders for holding small sponges or swabs, and one or two large sponges for quickly sponging out the mouth; they must be soft and free from loose bits.

A cylinder of oxygen for inhalation, with a tube for putting into a patient's mouth, or an aluminium funnel attached to hold over patient's mouth, if in a horizontal position.

The patient should be instructed to remove or loosen any tight clothing, corsets, waistbands, collar, or belts. Studs should be taken out. Some ladies wear a tight ribbon band for ornament round their necks: this simple matter might lead to the venous blood being held back in the neck. When in the chair the operator must see that artificial teeth, and anything loose in the mouth, are removed. The position of the patient's head in the chair should be in a line with the body, not far back or too forward. If they show marked signs of nervousness, direct them to clasp their hands, which has the effect of locking them when going under the influence of the gas. With men, place their feet on the floor each side of the chair. In any struggling which might ensue there is less chance of their getting such a firm purchase as if they were against the foot-rest.

The face-piece, which has a rubber pad around the edge, should be well inflated with air, but not too full. Whiskers, moustaches, and thin faces require some manipulation to prevent air getting in at the sides. The gas cylinders should be well turned on before the patient enters the room. The patient is allowed to breathe through the face-piece, with air for a few breaths, then the gas is let in and the patient is taking gas without air; but if, after several breaths, there is any sign of cyanosis a full breath of air now and again will rectify it. But the gas should be without air until the patient is fully under its influence, excepting anæmic and weak people, when, after a few breaths of pure gas, a small percentage of air may be permitted through the airway of the stopcock.

The administration may be summed up by a description of the physiological action of the gas and the signs of anæsthesia.

The gas passes through the mouth, past the tonsils, the pharynx and epiglottis, through the glottis, down the larynx, the trachea, into the bronchi, right and left, into the bronchial tubes of the lung substance, and then into the lobules of the lobes of the lungs. Dr. Halliburton, in his "Handbook of Physiology," states: "Each lung is partially subdivided into 
separate portions called lobes, the right lung into three lobes and the left into two; each of these lobes again is composed of a large number of minute parts, called lobules. Each lobe may be considered to be a lung in miniature. On entering a lobule, the small bronchial tube divides and subdivides, its walls become thinner and thinner until at length they are formed only of a thin membrane of areolar, muscular, and elastic tissue. At the same time they are altered in shape; each of the minute terminal branches widens out funnel-wise, and its walls are pouched out irregularly into small saccular dilations called air sacs. The air sacs or vesicles may be placed singly, but more often they are arranged in groups or even rows. Outside the air vesicles is a network of pulmonary capillaries spread out. Between the air in the sacs.and the blood in the capillaries nothing intervenes but a thin membrane. The object of the complicated structure of the lung is to provide a very large surface for the interchange of the gases in a compact organ. The total surface of the inside of the lung has been variously calculated, but it may be taken to be about go square metres in the adult, or about the size of a carpet necessary to cover the floor of a good-sized room, ro yards by I2 yards."

The gas passes through the membranous walls on the principle of osmosis, into the blood; here it is taken up and held in loose combination with the hæmoglobin of the red corpuscles which finally reach the cells of the brain, where its influence gradually paralyzes the parts of the nervous system-the cerebrum, cerebellum, spinal cord-until the medulla is reached, when the signs of anæsthesia indicate the limit to which we may go. If pushed and continued, an overdose will complete the general paralysis and death take place.

Generally speaking the stages of the action of $\mathrm{N}_{2} \mathrm{O}$ gas may thus be summarized:

First stage :

Tingling in limbs.

Patient feels exhilarated and breathes more quickly and deeply.

Consciousness becoming lost.

Respirations deepen and become more regular.

Pulse fuller and somewhat quickened; the temporal artery just in front of the ear is a convenient pulse.

Hearing becomes acute. 


\section{Second stage :}

Patient is conscious to anything being done, but is not really under. To attempt any extraction at this stage would produce shock or struggling.

Patient may make movements.

Excitement may occur, especially if air is admitted.

Respiration is now deeper and quicker than normal and becoming regular.

Pupils gradually dilating.

Complexion is dusky.

Eyelids twitch and slightly separate.

\section{Third stage :}

Respiration becomes automatic and regular.

Stertor caused by the aryteno folds of the larynx becoming approximated to one another and closing up the airway, the second or third stertor should be the limit of depth of anæsthesia and the gas withdrawn, in this method.

Pulse is rapid and not so strong as in second stage.

Muscular system is relaxed; patient's arm when raised will flop down when released.

Conjunctival reflex of the eye has gone; test this by touching it with the ball end of the finger.

Pupil well dilated usually.

The patient's face is distorted, the eyeballs rotate, or there is a vertical nystagmus or turning up of the eyeballs. Patient is also cyanosed, badly in some cases.

The signs of anæsthesia are stertor, flaccidity of the muscles, and automatic breathing. Neither of these signs is absolutely conclusive, but taken collectively they are reliable; but the anæsthetist by constant practice recognizes the phenomena when they arrive. If the gas is unduly pushed there will be jactitation, convulsive movements, and opisthotonos or arching of the back. These phenomena are very inconvenient to the operator.

The operator commences his operation and must carry it out quickly, as there are only thirty seconds of available anæsthesia. He must be careful not to push the tongue back or force the lower jaw downwards, both of which will embarrass the breathing. He must also be careful in operating on the lower jaw not to lacerate the soft tissues at the base of the tongue, as that 
organ swells up considerably under gas. He must also be prepared for a tooth springing out suddenly and lodging in the throat. These things can be avoided by having a finger behind the tooth or using Carter's spoon. In the upper the operator should be quite accustomed to having a small napkin always in his hand; this will not worry him when he is used to it. $\mathrm{He}$ will find it useful to be hanging in a suitable position to catch a tooth if it should suddenly slip. The patient should be allowed to come round without any fuss or hurry, so long as the colour is returning satisfactorily and breathing is all right. Silence must be maintained throughout the administration. The patient is liable to shock if he is hustled, as it were, into spitting out, and so on. The symptoms of asphyxia being absent and the colour good there is no need for anxiety; the patient is waking up and should not be alarmed. The gag which was put into the mouth before commencing should be left in place until the patient is sufficiently conscious to know that we are removing it. Neglect of this precaution will sometimes make the patient declare that he felt everything. A bowl should be placed to his lips, and an instruction given to spit the blood out. A glass of water is then held to the lips for rinsing out.

Oxygen is also given in the short method of gas administration with Dr. Hewitt's apparatus, and his book on "Nitrous Oxide and Oxygen for Dental Operations" is very instructive as to the advantages with this method in order to reduce the asphyxial disadvantages with pure gas only.

During the early years of the use of $\mathrm{N}_{2} \mathrm{O}$ gas various endeavours were made, first, to reduce the asphyxial factors of gas, to which Sir F. Hewitt contributed a very great share. $\mathrm{He}$ proved that $\mathrm{N}_{2} \mathrm{O}$ gas was a true anæsthetic and acted on the brain centres irrespective of the deoxygenation of the blood, because by using oxygen he could produce non-asphyxial anæsthesia. Secondly, as the question was accepted as settled that $\mathrm{N}_{2} \mathrm{O}$ was non-asphyxial by the use of air or oxygen, then why not administer it continuously? This was a logical extension of its use, but the difficulties were great to devise an apparatus, which would necessarily have to be perfect, for conducting the continuous administration of a gas which, being devoid of free oxygen, exhibited asphyxial symptoms under a short administration. However, by the patience and ingenuity of many enthusiastic dentists and medical men, the points of 
difficulty have been overcome. Credit is due to such men as Paterson, Coleman, Hilliard, Hewitt, Teter, and others. Had Sir Frederick Hewitt been spared he probably would have continued his voluminous and successful investigation to the prolonged nasal method.

Dr. C. K. Teter, D.D.S., of Ohio, U.S., is an acknowledged authority on nitrous oxide gas and oxygen. From his writings in the Dental Cosmos we are indebted for a clear enunciation on the subject (see Dental Cosmos, I907, p. II40). He describes an opportunity he had of observing the actual effects of gas and oxygen on the tissues of the brain in the case of a boy who fell on his head, causing an epidural hæmorrhage. The pupil of the right eye was extensively dilated, and the muscles of the same eye were paralyzed from the pressure on the centres within the brain. He became unconscious almost immediately, and remained in that condition for about twelve hours before an operation was decided upon. His condition was very grave, and little encouragement was given as to his recovery. Nitrous oxide and oxygen was decided upon as the safest anæsthetic to give in this case. An opening was made in the skull and by delicate manipulation the brain was moved to one side and a large amount of clotted blood removed. After giving the details of his administration he goes on to state: "This operation gave me a long-wished-for opportunity to observe the human brain under anæsthesia and to notice the effects of nitrous oxide and oxygen upon it. I made the following observations:

" On decreasing the oxygen slightly for about five seconds I noticed a quick change in colour and a perceptible dilatation of the brain. This was not carried very far owing to the probable injurious effect of such a procedure. On increasing the oxygen to almost one-half of the mixture (50 per cent. gas $+5^{\circ}$ per cent. oxygen) it was but a few seconds, not more than nine or ten, until the brain assumed its natural colour and returned to its normal position. The rapidity of the action was a revelation to me and called forth some expressions of surprise from the attending surgeons. I can say without reserve that the dilatation and congestion here noticed were not due in the least to the anæsthetic action of the nitrous oxide, but the condition was entirely an asphyxial manifestation. The recovery of this patient was wonderful. The surgeons did not expect that he would regain consciousness for at least six or seven hours, but 
to their surprise he became conscious two hours after the operation and made very rapid recovery, regaining all of his faculties and usual vivacity." Dr. Teter also observes: " It is interesting as well as instructive to observe the effect of nitrous oxide and oxygen upon the brain, and to note the difference when nitrous oxide is administered with and without oxygen. When the nitrous oxide gas is administered alone we find that as soon as the asphyxial element begins to enter into the procedure the brain loses its natural pink colour and turns more or less gradually to a dark purple. If the administration be continued, without air or oxygen, it will take on an appearance resembling that of stagnant blood. As this discoloration progresses there is a dilatation of the brain, and the greater the discoloration the greater the dilatation, so that it will protrude through an opening in the skull. One can imagine what this would mean to a patient with a myasthenic heart, or one with apoplectic tendency. This accounts for the headache complained of sometimes after the administration of an anæsthetic. The condition of the brain is altogether different when a patient is anæsthetized with oxygen in combination with the nitrous oxide. I speak from actual observation, as it has been my good fortune to observe the brain while the patient was under the influence of this mixture."

We have made enquiries for a book by Dr. C. K. Teter on this subject, and have been told that he has published nothing in book form. However, his various writings which have appeared in the Dental Cosmos from time to time are most instructive. Dr. J. T. Gwathmey's work on "Anæsthesia," which is an excellent volume, contains a description of Dr. Teter's method of administration: This, studied with Sir F. Hewitt's work on "Nitrous Oxide and. Oxygen for Dental Operations" and his work on "Anæsthetics" will give any student a competent and comprehensive knowledge of the subject which would stand him in good stead in practical dental work.

The experimental stage of testing the practical value of the prolonged nasal method of administering $\mathrm{N}_{2} \mathrm{O}$ and oxygen has well passed and its position satisfactorily secured. It has been established as a definite and satisfactory way of carrying out dental operations, including extraction of teeth, opening up roots with extraction of live pulps, and preparing cavities for 
filling. There are many men in the Incorporated Dental Society who have been using this method for over seven years with success. Of course, it means that two men who are thoroughly

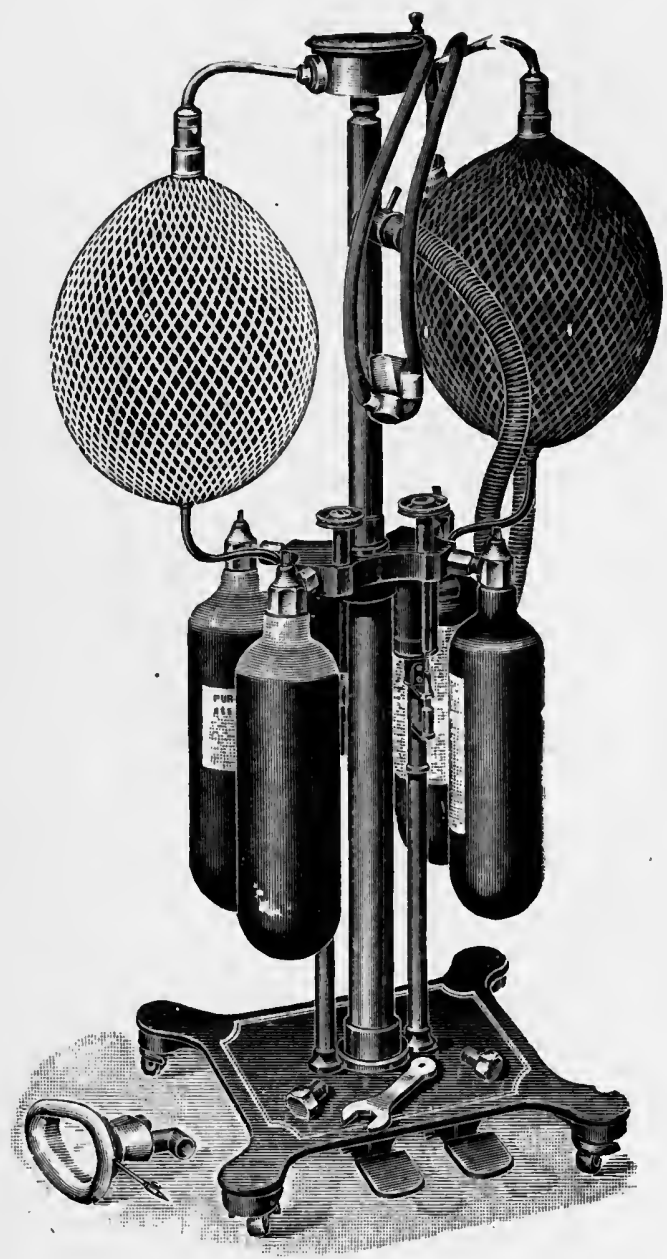

Fig. 46.-The Moylan-Jones $\mathrm{N}_{2} \mathrm{O}$ Gas and Oxygen Apparatus.

experienced must co-operate, and even a third assistant be present to manipulate anything that may be required during the operation.

, Mr. W. J. Moylan Jones has spent many years in . 
practical investigation of this subject and the outcome of his work has been the production of his apparatus, which deserves great credit because of the simplicity and certainty of its service. The principal features of his apparatus are the

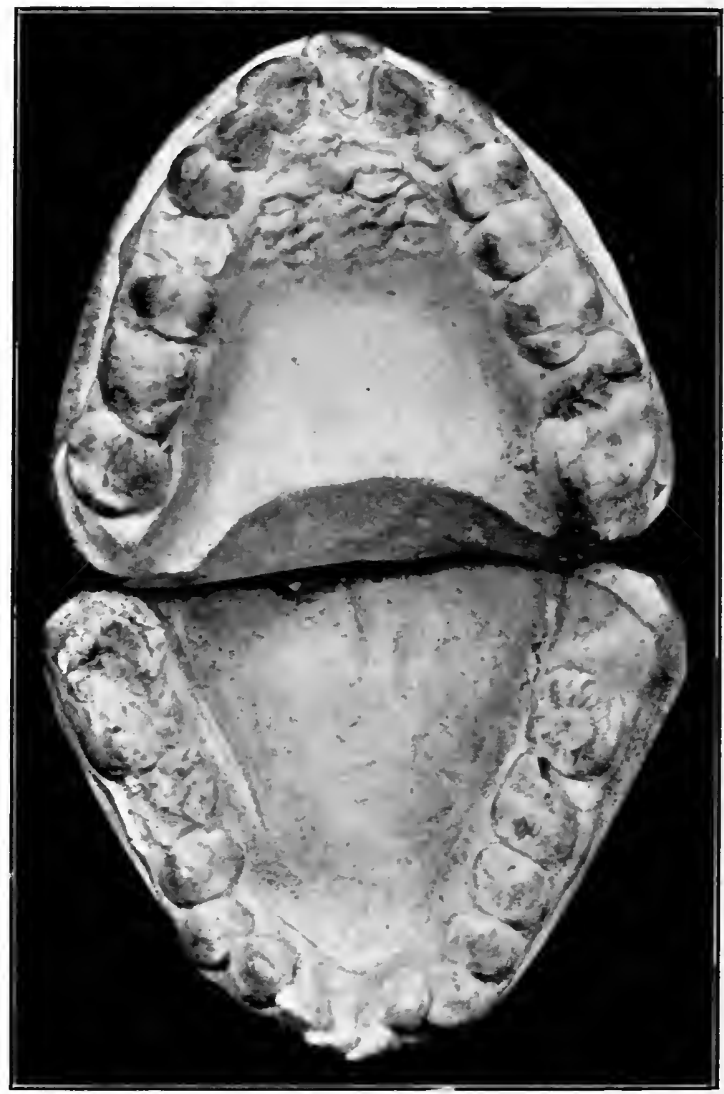

Fig. 47--Models of Patient's Mouth taken before Extraction of TEETH SHOWN IN FIG. 48 .

nasal inhaler and the cylinder, which consists of a fan which thoroughly mixes the two gases, having an entirely different specific gravity. To insure the gases being mixed at the moment of breathing, the fan is set in motion by the patient's own act of breathing. The construction of the fan is arranged so that there is not the slightest resistance. On the top of the 


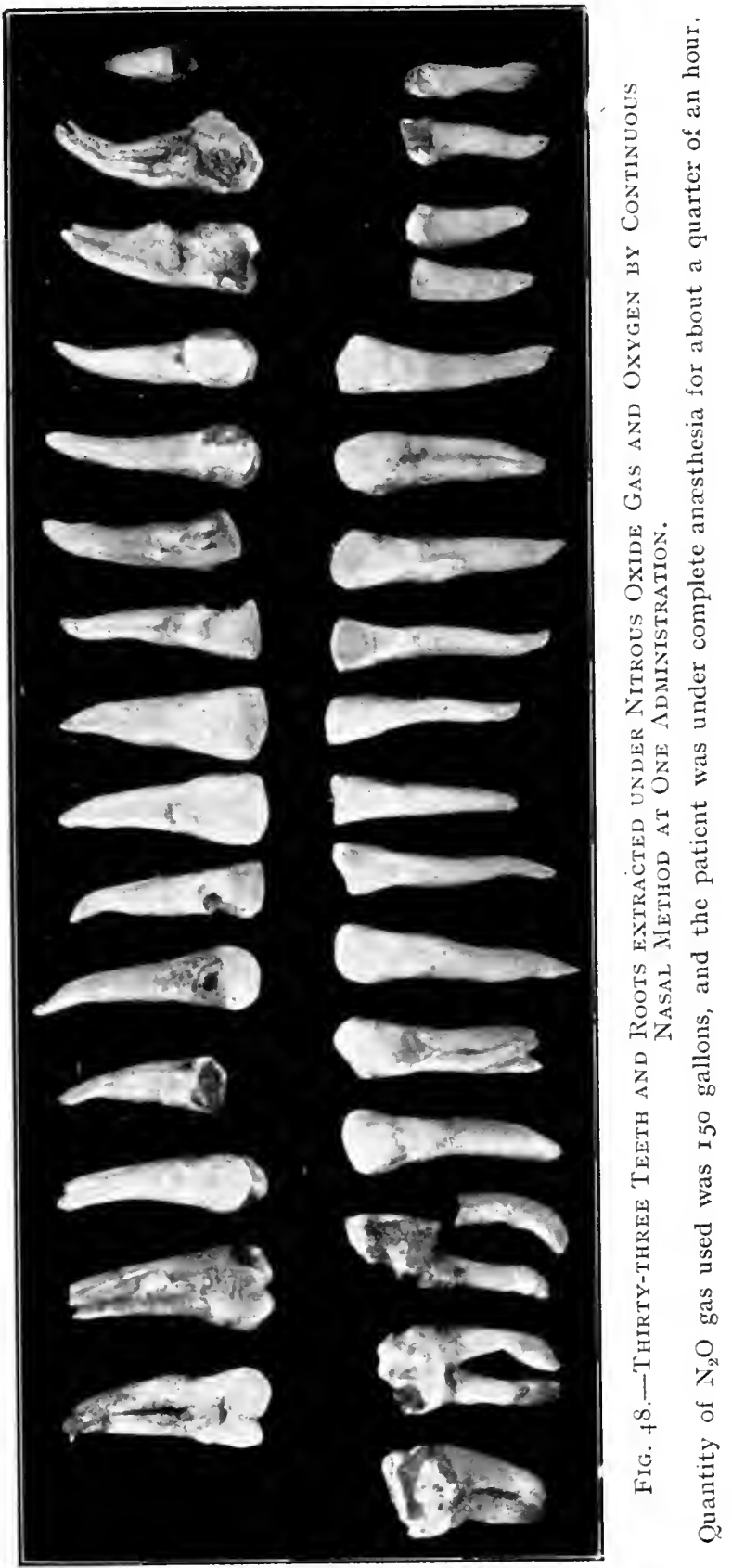


cylinder is a carefully worked out gauge, which, by turning a neat handle, registers the working of inner cylinders containing channels that deliver the gases as required in the central chamber where the fan is working. If the operator requires air it gives

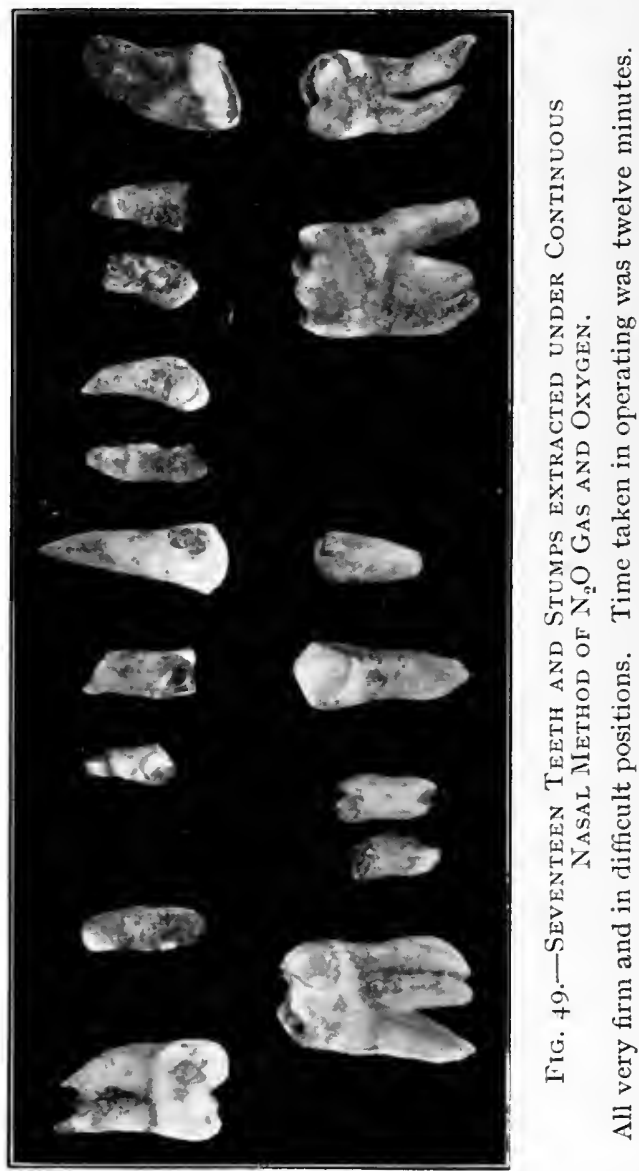

the proportion desired. When $\mathrm{N}_{2} \mathrm{O}$ is wanted, it gives the additional proportion desired up to full percentage. When oxygen is required the percentage -of gas and oxygen required is delivered and registered up to full percentage of oxygen, while the percentage of $\mathrm{N}_{2} \mathrm{O}$ is at the same time lessened to zero. The cylinder automatically subtracts or adds the proportions 
required instantly. If rebreathing into the gas-bag is desired, it can be done by turning a valve in the feeding-tube. With this method great pressure is sometimes required to keep up the anæsthesia. This also has been arranged for by nets which become tight, and the size of the bags draws up a ring which automatically squeezes them. Mr. Moylan Jones has demonstrated the certainty of its working many thousand times, and the writer has operated with him in hundreds of cases. The illustrations of two selected cases are here given to show the practicability of this method and its obvious advantages to both

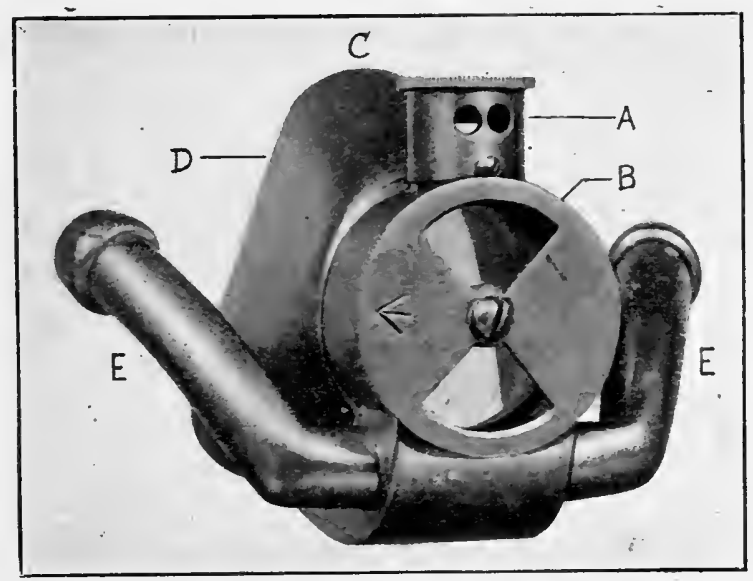

Fig. 50.-The Moylan-Jones Nasal Inhaler for Nitrous Oxide GAS AND OXYGEN.

A, Exhaling valve; $\mathrm{B}$, air valve; $\mathrm{C} D$, rim which fits any nose; $\mathrm{E} \mathrm{E}$, tubes for free supply of gases.

patient and operator in saving time which were not in any sense exceptional, both being young women of fragile build and very nervous (see Figs. 47, 48, and 49).

The nasal inhaler shown in Fig. 50 has some simplified practical points. Air can be admitted as desired. The rim will fit any nose without a rubber pad. There are very occasional noses with a very narrow bridge, but these are easily remedied with a small piece of cotton-wool. When the air-valve is closed the respiratory valve in the chimney works automatically and indicates the patient's breathing. It can be thrown out of action by turning the milled head as desired by the anæsthetist. 
The nose-piece is held firmly in position by a simple clamp at the back of the head.

The patient is prepared as described for short gas, and the clothing must be well protected from blood as there is considerable mopping out to be done sometimes, especially in pyorrhœa cases. The patient is then directed to breathe through the nose only, and may have to be reminded; a few breaths of air may be allowed before closing the air-valve. Attention is now given to the cylinder gauge by turning the handle to 25 per cent. air and 75 per cent. gas-one movement does both; another few breaths, then to $5^{\circ}$ per cent. air and $5^{\circ}$ per cent. gas, then on to full gas. As the patient breathes through the nose satisfactorily, the mouth, being open, syphons in a small amount of air, and in patients of ordinary type the administration goes on until the anæsthetic effects are showing themselves. There is no need to hurry, as the principle of a long induction brings the system gently under the true anæsthetic action of the $\mathrm{N}_{2} \mathrm{O}$ and a longer anæsthesia results. It is particularly applicable to this method. When the signs of anæsthesia are approaching, the handle can be put to 5 per cent. oxygen and 95 per cent. gas and kept there, provided that the patient shows no sign of excitement. In the meanwhile, if the patient should persist in breathing through the mouth after being reminded, the mouth cover is adjusted, and if necessary the nasal expiratory valve is turned off so that the patient receives the gas through the nose into the throat and respires through the mouth-cover valve. There is a little difficulty just here in a few exceptional cases where the soft palate is unusually long and the pressure of gas through the nose keeps it forced down on to the tongue so that it acts as a valve, preventing the expiration of the patient's breath. The momentary removal of the nose-piece now and again will remedy this. The patient continues to breathe 95 per cent. gas and 5 per cent. oxygen as long as it is suitable to the indications. The oxygen is not used as an anæsthetic in any sense, but is instantly at hand if the indications of the patient's face, ears, lips, and finger-tips, and also the breathing require it. It must be noted that the patient has a certain amount of oxygen kept in the lungs and blood for a while as the interchange of gases in the blood and lungs involves a percentage only (see chapter on Gaseous Interchange of the Blood). As the administration 
proceeds, oxygen will be required accordingly, and at its conclusion the patient's lungs can be flushed with pure oxygen according to the condition of the patient and the time occupied. The signs of anæsthesia are automatic breathing, slight snoring stertor, loss of conjunctival reflex, and flaccidity of muscles. Any symptom which is presented in short gas can be almost instantly removed by the oxygen. The skilful anæsthetist does not jump about with large percentages of oxygen, but by constant experience he knows pretty well how to adjust a percentage to the patient in hand. Symptoms must be watched for and anticipated by a few seconds, which allows time for the oxygen to take effect. The anæsthetist should be a man of cool temperament, self-confident, resourceful, with a keen, trained eye, and sympathetic yet firm manner. The patient is keenly sensitive to the transmission of psychological factors, and the anæsthetist should be quite capable of impressing a patient with his own confidence of the success of the administration.

Then there are some patients who require a different administration of the gases.* A strong robust person with an excitable temperament will, of course, tax the ingenuity of the anæsthetist. Gas is turned on and there is a very slow response to its action; the mouth is then covered with the hand, a rubber cover, or a celluloid cover fitted with an expiratory valve (the latter is preferable). This should bring the anæsthetic effects into play, if not deeply enough; then the expiratory valve of the nasal inhaler is turned off and the gas is forced through the nasal passages under pressure from the bag which has to be consistently kept up until the signs of anæsthesia are reached. The time of induction varies from one to three minutes. With robust patients this procedure usually suffices, but with alcoholics and very robust men it is sometimes necessary to stop the inhalation and start again by using the face inhaler, as in short gas, and changing easily and quickly, when the patient is well under, to the nasal inhaler. If the patient cannot be kept under, this method has the advantage of extending the period of short gas. Also, if there is the usual bad cyanosis which accompanies alcoholics it can be dealt with on the spot with full oxygen.

* One hundred-gallon up to $25^{\circ}$-gallon cylinders of $\mathrm{N}_{2} \mathrm{O}$ gas and 3o-gallon and 6o-gallon cylinders of oxygen must be employed in order to secure sufficient pressure. 
The mouth cover is removed and the patient has to be conducted through a narrow path between anæsthesia and partial recovery. The difficulty, usually, is to keep him under. The

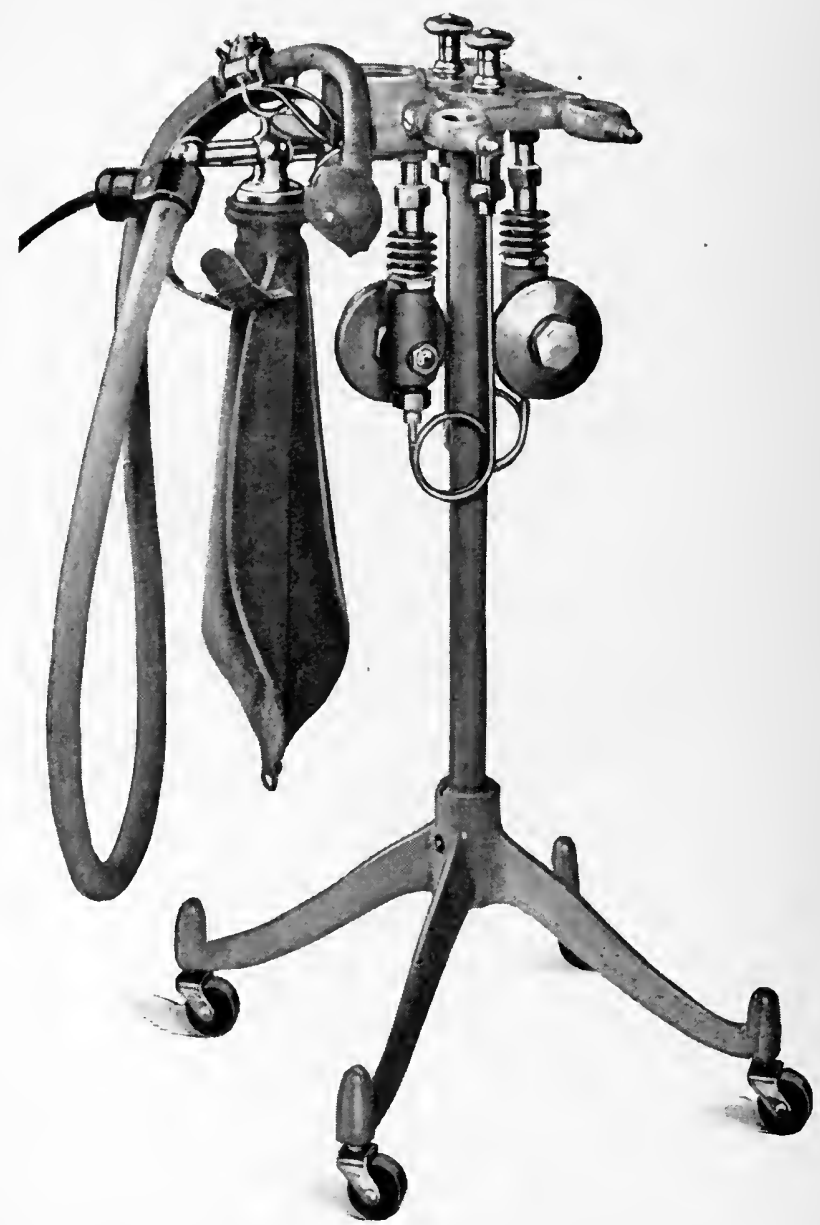

Fig. 5i.-The S. S. White Nitrous Oxide Gas and Oxygen Apparatus.

patient is kept free from signs of asphyxia by using the oxygen 5 per cent. to 25 per cent. usually, but higher occasionally, or perhaps a full breath of pure oxygen. The apparatus is capable of instant ajustment according to the type of patient.

Anæmic and weak people and children are good subjects, as 
they can easily be kept under with a minimum of danger, which can hardly be said of short gas. They readily respond to air or oxygen. Some writers emphasize that the patient is only safe when the tips of the ears, nose, lips, and fingers are a rosy pink. While this is admitted, yet there can hardly be a fast line in actual

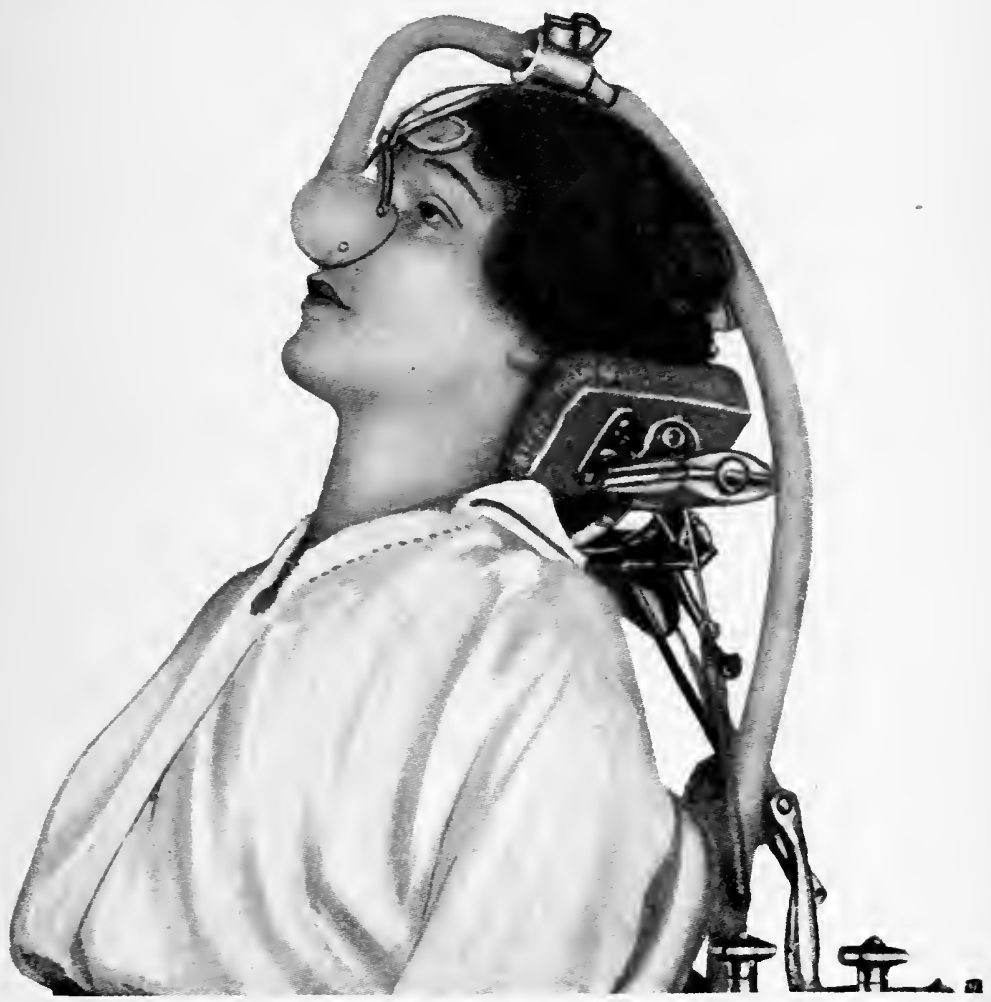

Fig. 52.-The S. S. White Nasal Inhaler No. 6 adjusted, Showing Closeness of Adaptation to THE Face; also showing how the WEIGHT OF THE INHALER TUBING IS SUPPORTED TO PREVENT DRAGGING.

practice. The nature of the breathing is the main factor. Patients with a weak heart or lungs take the mixture well, such as those with arterio-sclerosis and mitral trouble. Great care, of course, is imperative, but they give no trouble if it is carried out skilfully. The use of oxygen requires intelligent therapeutic application because there is danger in unnecessary depression and excitement of the medullary centres. 
Another apparatus that is being used with equal success is the S. S. White nitrous oxide and oxygen equipment. The administrations described can be carried out with similar adjustments, although the mechanical parts of the apparatus are different. The nasal inhaler is ingenious, simple, and well arranged. It may be adjusted to any shaped nose by easily bending it to make it airtight. The nasal inhaler is securely clamped to the head. The valve body, which is placed on top of the patient's head, is within easy reach of the anæsthetist, who usually stands at the back of the chair (see Figs. 5I, 52, 53, and 54). It consists of an exhaling valve and an air valve,

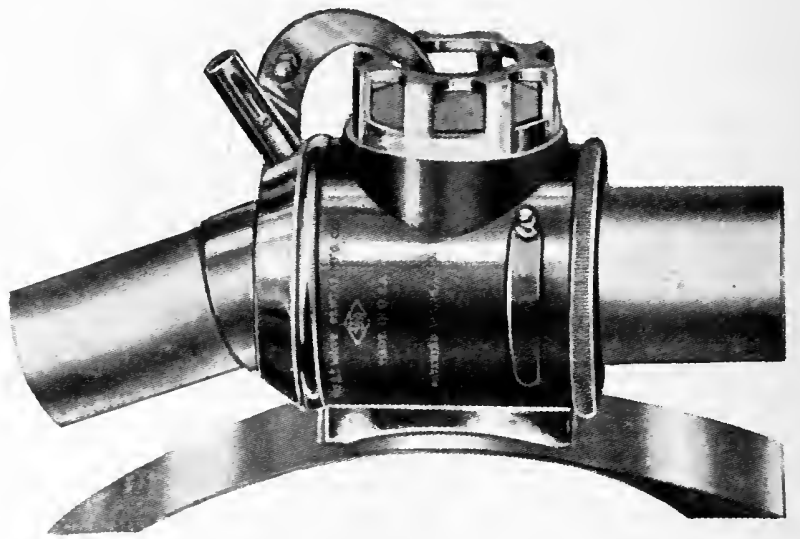

Fig. 53.-Valve Body of S. S. White Nasal Inhaler No. 6 with Exhaling Valve and Air-Valve.

which permit of similar adjustments for controlling the patient's breathing. There are two gauges which indicate separately the amount of gases being used. Each apparatus has accurate devices for the separate control of the $\mathrm{N}_{2} \mathrm{O}$ and oxygen, a perfected means for thoroughly intermixing the gases, and inhalers can readily be changed from one to the other.

The advantages of the nasal method are so obvious in practice that it is somewhat inhuman to submit a patient to short gas for any tooth that is likely to be difficult to extract. There is also the operator's moral responsibility to his patient not to give him any pain, as the experience of coming round to an incompleted operation is often worse than having no gas at all, because he has not the mental power to bring his will to bear the 
pain. He is certainly psychologically unprepared for it, for his mind was impressed with the mental suggestion and guarantee that he would feel no pain.

With reference to accidents, they are very rare if the operator is careful to avoid pushing the tongue back, bearing the lower jaw down, and injuring the tongue. He must be careful to mop the blood out as he proceeds. This is important, because not only is there danger of a blood-clot entering the larynx, but

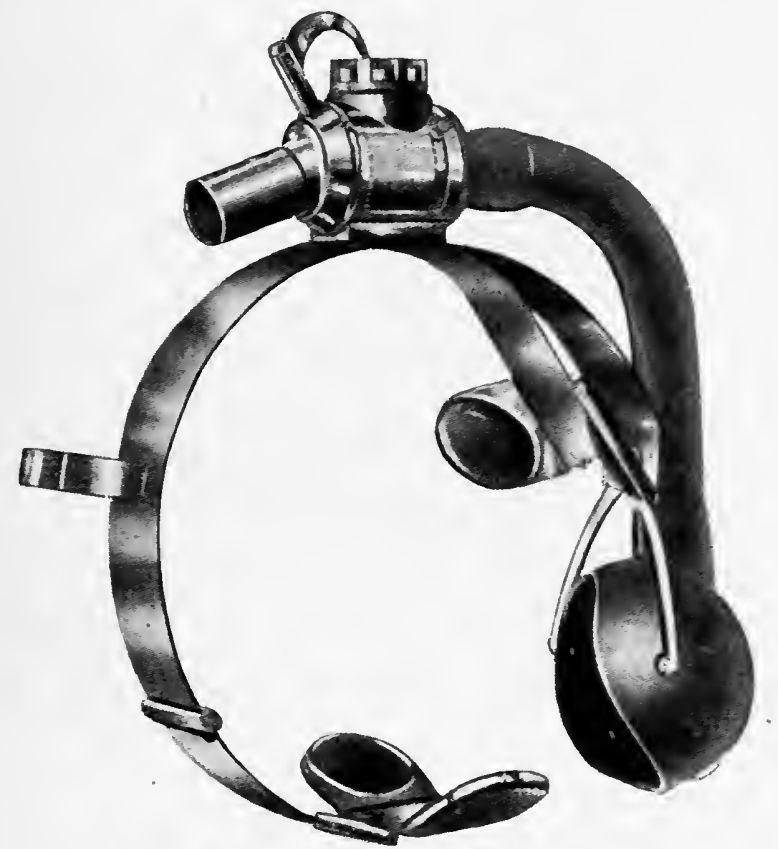

Fig. 54.-S. S. White Nasal Inhaler No. 6 for Prolonged Analgesia or Profound ANesthesia.

the irritation caused by its entrance might set up spasm of the glottis. This condition is best treated by pushing the patient's head forward deliberately, which empties the throat of blood, as well as the mopping out. The writer has a pile of separated aseptic napkins handy and also rough pieces of Turkish towelling, which cover a large surface and soak up quickly. A large soft sponge, without loose bits, is very useful and quickly entangles clots and brings them away; also small swabs or sponges fixed on a sponge-holder for mopping the throat itself if necessary. 
The noise of the gurgling of the blood will indicate the condition, but the routine mopping out will obviate the obstruction of blood in the throat. In very rare cases the patient's breathing may stop and signs of asphyxia be present, such as eyeballs being very prominent, lividity, and cyanosis, accompanied by rigidity of the chest-walls. Clear the mouth quickly of blood, pull patient's tongue forward, and forcibly so, if it continues, with a traction movement. Pass your finger well down the throat to clear away mucus or blood and pull the tongue forward at the base. Apply pressure on the diaphragm and lower walls of the chest to start breathing again. If done intelligently this is an effective form of artificial respiration. If two men who understand their work co-operate, there is no difficulty in attending to this, but it must be done coolly and quickly. If these measures should not be successful the patient must be lowered in the operating chair to a horizontal position with the head lower than the feet to allow the blood to reach the medulla, and artificial respiration recommenced. This is effective, as the movements of the ribs and diaphragm react on the lungs to inflate and empty them, and the thoracic pump is restored which assists the heart's action.

There are several methods of applying artificial respiration, but the usual one is Sylvester's, which consists of depressing and raising the patient's arms alternately. The arms are gripped at the elbows and well pushed against the chest-walls to empty the lungs, then they are slowly drawn outwards and upwards; which thoroughly opens the chest cavity like a pair of bellows. These movements are carried out at the rate of about fifteen times to the minute. Stoppage of breathing must not be confounded with patients holding their breath. This can be tested by the presence or absence of the conjunctival reflex.

Syncope in severe cases can be treated by an hypodermic injection of ether 30 minims or strychnine sulphate $\frac{1}{60}$ grain. Oxygen is the main thing, and it is very rare for any other therapeutic restorative to be required. 


\section{CHAPTER XVI \\ THE EXTRACTION OF TEETH}

THE operation of extraction of teeth is one which is sometimes attended with some degree of uncertainty and also difficulty. While the great majority of teeth come out without much difficulty, and free from post-operative incidents, yet the experienced operator is sometimes confronted with cases that tax his abilities, either in the operation itself or the sequelæ that arise. The operation depends upon a few important principles:

I. The form or shape of the roots of the teeth.

2. The density and substance of the bone in which the teeth lie.

3. The position of the roots in the bone.

4. The build or construction of the instruments.

5. The operator's intuitive skill.

6. The prevention of accidents.

7. The treatment of accidents and sequelæ.

The teeth, which consist of crowns and roots, present an abundant variety of forms, shapes, sizes, and substance. The tooth to be extracted may give us some idea as to the means which we may adopt for its complete removal. The extraction must be carried out in the line of least resistance, for herein lies the greatest likelihood of the tooth coming out without fracture, either of itself or the bone in which it is situated. There is no possibility of classifying all the various movements necessary to the successful withdrawal of all roots, because of the variety of forms and condition of many of them. The movements which might be applied to a normal tooth might be quite inapplicable to the same tooth if it were in a broken-down state. Also, many of them have a subtle twist in their general shape, especially upper canines and molars. Teeth with crowns which appear short, thick, and stout are usually long in the roots; long, bulbous 
crowns are often short in the roots; while crowns that are flat on their internal and external surfaces are long in the roots and well implanted in their sockets.

The density of the bone is another factor in successful extraction. In some cases the bone is soft and cancellous, so that the thin blade of a sharp instrument cuts its way quickly; care is required to avoid damaging it and the vessels contained therein. In other cases the bone will quickly respond to the insertion of the forceps blades by dilating nicely, and although it is not soft, but, on the contrary, usually tough, yet there is an elasticity present which allows of the successful oscillation of the tooth from its socket. But there are cases where the bone is quite unyielding, and where forcible dilation will result in its fracture. Bone is more likely to give when several teeth are standing together, but where a tooth is standing alone and isolated the resistance is much greater.

The position of the roots of the teeth and the corresponding position of their sockets, or alveoli, in the bone of the jaw are the next important factors. If the ordinary movements applicable to a given tooth fail to release it, we apply ourselves closer to our task and bring our experience to guide our movements. Experience has taught us to observe that individual teeth have a tendency for their roots to lie in a certain direction, which in some instances is very much accentuated. This accentuated individual inclination to certain shapes applies to nearly all the teeth, upper and lower, from the canines backward. Those which give us the greatest difficulty are the upper canines, first bicuspids, which are usually bifurcated, and the upper six-year-old molars, the lower canines, six-year-old molars, and lower third molars.

The instruments used for extracting teeth are quite an important part of the business; they must be built for their work. They have two ends; both of them are important: one is for grasping the tooth at the cervical part, and the other end is for the operator to grasp. It will readily be seen that a pair of forceps has two important functions, for which the instrument maker is responsible: one is its ability to grasp and the other its ability to be grasped. In both these functions their proper dynamic relation concentrates the operator's power with an economical reserve. Let us examine this important point. If we take a lower molar for our consideration, the blades should 
be so modelled that they will accurately embrace the surface of the part they cover. Some of the forceps blades made forty years ago were beautifully modelled, and, after years of wear, are still in good working order, showing that the individuality of the instrurnent maker is the all-important and valuable part of the instrument. Our instruments are beautifully made to-day compared with those of forty of fifty years ago, but there were a few instrument makers at that time whose individual work was truly an art. Their forceps can be reproduced, as far as their blades are concerned, but they sometimes lack full efficiency because the handles are not properly or suitably shaped. Size and weight indicate nothing excepting an unwieldy power which will smash everything it is applied to. The operator's hand in grasping the handles is equally as important as the blades which grasp the tooth. While the manufacturers have produced extracting forceps in a thoroughly efficient manner, yet the points we are insisting upon can hardly be universally produced in a given shaped instrument. There are many operators who do all their extracting with halfa-dozen pairs of forceps, or less, as their stock. But even in these cases the efficiency of the instruments depends upon these points. If we come across a pair of forceps which appeal to us, after having had some experience in extracting, it is worth while to have the blades of that pair reproduced with the handles of another pair that suit the grip of our own individual hands. At the same time the build of the handles which suits our grasp must be arranged by the makers so as to preserve the capability of the whole instrument of transmitting the line of force along the axis from our contracted hand to the position of the axis of the root to be moved. The dentist, as we have already remarked, has to be a man of many gifts. Here he is called upon to apply mechanical principles for the removal of a tooth which must be done completely and with a minimum of damage to the investing or surrounding bone. This is a true test of skill. It is the individuality behind the instrument which really matters, or, to familiarize a popular phrase, " it is the man behind the gun which counts." To review the operation at this point, it consists of anatomical considerations and the selection of suitable instruments already described, and now comes the operator's ability of applying the latent power of the instruments with anatomical accuracy, coupled with mechanical ingenuity to allow for or correct the digression necessary to remove the roots. 
There are generally accepted movements laid down for the removal of the various teeth contained in the dental arch. It must be understood that, while these movements are obviously correct in considering the diametric shape of the various roots of the teeth and their corresponding sockets with relation to the weakest wall, which is likely to give and allow for their loosening, yet these arbitrary instructions will by no means make up for lack of knowledge such as we have outlined as being the principles on which extraction should be carried out.

There are three stages in the removal of each tooth:

I. The seizure of the tooth.

2. The disentanglement of the investing membrane of the socket.

3. The withdrawal of the tooth.

The general movements for each tooth are-

Upper Centrals and Laterals.-Apply the forceps beak well up on the lingual side and then on the labial side applying pressure in the direction of the long axis of the root. At the same time traction is applied with a rotary movement. Care must be taken with laterals, as they are frail.

Upper Canines.-These require different procedure. The tooth is very much longer and stouter as a rule than the incisors, and often has a curve at the upper end of the root towards the bicuspids. It is situated in the solid portion of bone at the corner of the arch, but its position is approximately such that the external surface of the root is practically parallel to the outer surface of the bone. In consideration of these conditions a reserve of force is called up in the forearm ready for careful application as required. A pair of forceps with strong yet well-tapered and sharp blades should be used. Force the blades well up for two reasons: firstly, we get an impetus on the palatal side, as the outside wall of the socket being very weak, we can safely get a leverage at this point, and also the bone on the inside wall is strong; secondly, the labio-buccal or outside plate of bone very often comes away with the tooth, as the periodontal membrane is usually exceptionally adherent with this tooth, and the blade of the forceps should overcome this as it otherwise adds to the resistance of the extraction. The movement should be outwards, then inwards, with the object of oscillating the apex of the root outwards, as the outer plate of the bone is thin. These movements carefully, 
deliberately, and patiently applied, together with a vibratory movement of the forceps, will loosen it. If the canine is standing alone, and there is great resistance, apply the blades to the sides of the tooth with a vibratory movement, which will often loosen it.

Upper First Bicuspid.-The same movements as for canines, but care must be taken with rotary movement, as the root may be bifurcated and break at the apical part. Not so much force is required as for canines.

Upper Second Bicuspids.-Same movements as for first bicuspids.

Upper Six-Year-Old Molars.-Apply the point of the outer blade between the two buccal roots and the inner blade to the palatine root, drive up to securely embrace the neck of the tooth, apply pressure and at the same time traction in a line with the axis of the palatine root, then slight inward movement, until loosening takes place, then slight twisting movements are allowable to disengage it from the socket. This tooth usually requires considerable force.

Upper Twelve-Year-Old Molar.-Same movements but less force required.

Upper Wisdom Molar.-This is usually an easy one to extract, but requires care. It is usually moved by outer traction. Caution must be taken to prevent it jumping out suddenly and falling into the throat. Stump forceps such as Mr. Thorne's are useful for this tooth.

Lower Centrals and Laterals.-Slight downward movement and outer traction will usually move them, but the gum is apt to adhere badly; when loosened, a quick rotary movement will disengage them. Care must be taken so as not to tear the gum badly: tightly pinching the gum will usually prevent this; if necessary, use gum scissors and cut the gum.

Lower Canines are difficult to move sometimes, and require considerable downward pressure and outward traction and a slight inward movement until loosened.

Lower Bicuspids are not difficult and are apt to jump up suddenly through the forceps blades. An outward and inward movement will dislodge them.

Lower Six-Year-Old Molars require considerable pressure and firm, deliberate movement. Well apply the blades between the mesial and distal roots, then drive them down. These teeth 
are somewhat similarly situated as the upper canines, the inner side of the bone is thick and dense, and a good point on the inner blade will penetrate this dense bone at the point where the division of the roots occurs. The outer plate of the socket is also liable to come away, as with the upper canine. An inward movement followed by a firm outward movement is applied until the tooth loosens. Owing to the roots often diverging, the tooth has to be brought away, from the side. The apical portions of the roots also curve backwards and a rotary pressure is applied synchronously, otherwise fracture will occur. Most operators prefer hawk's-bill lower forceps, which admit of this movement.

The Twelve-Year Lower Molar is easier to remove, as the roots usually converge at the ends.

The Lower Wisdom is sometimes more difficult to remove than the upper one. Usually it is not very difficult; on the other hand, it sometimes presents a serious problem to the operator owing to the dense bone in which it is situated. The difficulties of extraction usually appear with badly decayed and broken-down teeth and stumps. The difficulty is intensified by the fact that we are not justified in risking a severe fracture of the bone, which might prove to be a serious complication, and even with the risk the tooth might not be removed. We must realize the nature of bone; it appears to have a low power of recuperation, hence necrosis is an ever-present possibility. The writer has come across several cases of necrosis of the jaw in practice. One case was very serious, where an operator had endeavoured to extract a lower six-year molar, and before six months had elapsed ten other lower teeth had to be removed, together with huge pieces of necrosed bone, as a consequence of fracture in applying force to remove the first tooth.

The really difficult cases are usually, upper canine roots, upper broken-down six-year molars, and upper bicuspid roots, lower canine roots, broken-down six-year molars, and broken-down third or wisdom molars. For an upper canine root the forceps must be sleek, sharp, and strong. What slender hold there may be must not be lost by pulling; the better way is to avoid traction until the root is loosened and carry out the restricted movements which a broken-down stump will necessarily only allow. The principle of moving any single root which cannot be properly grasped is to use prudent pressure, restricted inner and outer movements, and a twisting movement to jar the root away from 
its socket. There is no need to be nervous in applying pressure. The reserve power is gathered in the operator's forearm and is in readiness to be applied gradually; only sufficient power is used that is necessary to loosen it and no more, otherwise the power suddenly becomes force, which is destructive. The superfluous force, being suddenly released, will instantly cause fracture. In some mouths the whole of the anterior portion of the maxilla is in a resilient condition, and suddenly applied uncontrolled force would fracture it right round. Again, in a cleft palate case with hare-lip this condition may be very marked, and it would seem as though the pressure reasonably necessary to extract a molar must break away the whole side of the jaw. The parts must be well supported by the fingers of the other hand. Care also must be exercised if an apical abscess is under the root, which would cause a serious complication in the maxilla if the root disappeared into the antrum. While accentuating the necessity of continued pressure to maintain the hold on a slender root while loosening it, it must be distinctly understood that it is the dexterity of the pressure being in reality a part of the movement necessary to - loosen it; the remaining part of the movement being a vibratory twisting motion of the forceps. It does not amount to a definite movement. Observation of teeth in dry skulls will show that this jarring will move the tooth upwards. To illustrate the dynamic principle involved we can place some square nails head downwards with a casting ring round and then pour some molten zinc over them. When cooled apply the principles of stump extraction. The only movement that will be found to be successful is that of enticing the nails from their impacted position to where the surrounding pressure is less, and jarring is the only movement possible. Definite rotary movement is impossible, because the nails are square, but the rotary bias will do it. There is an engineer's axiom that if there is not room for two wedges in the same socket, jarring or tapping one wedge will drive the other one out. This axiom well applies to our subject.

The upper six-year molars, when broken down, sometimes require a deal of ingenuity. The anatomical position of the roots of this tooth being understood, several ways of dealing with it may be tried. A pair of stump forceps with one blade applied to the mesio-buccal root and the other on the palatine root, using restricted inward and outward pressure, or, a pair of sleek stump forceps applied to the buccal root only will sometimes move it by 
applying one blade to the buccal side at the corner of the division of the buccal roots, and the other blade to the palatal edge of the buccal root with steady parallel pressure on one side, and then the other, with patience, until it loosens. Splitting forceps will work well in some cases; these are used in order to separate the buccal roots and isolate the palatine root and bring it away. The palatal blade is well applied and then the outer cutting blade, with a safe step to it, is placed well up between the buccal roots. Gradual pressure is applied, when the cutting blade will gently cut through the soft bone, squeezing the buccal roots and usually loosening them; the blades then closely hug the palatine root, which will then come away. The buccal roots may now be lifted out with stump forceps. Lower broken-down six-year molars are sometimes readily removed by using straight-handled cow-horn forceps. The inner blade will find its way down between the mesial and distal roots; the outer blade is applied in a similar way. The forceps are not allowed to close tightly, but with the operator's thumb between the handles the beaks are allowed to slide under, while a firm rocking movement is made which works the tooth upward. Stump forceps applied to either root will sometimes bring the tooth away. Broken-down molar roots may readily be moved by the use of an elevator, but great care must be taken to support the part with the fingers and thumb of one hand, and with the straight elevator the forefinger must be laid along the shaft so that if it should slip it cannot go farther than the forefinger will allow; the alveolus may be used as a fulcrum, but the thumb should bear the greater part of the pressure. Lower wisdom teeth sometimes present serious difficulty in extraction, especially when they are in an impacted position. They may be situated nearly horizontally or in a vertical position. It is necessary in some cases to extract the twelve-year molar in order to secure necessary room for bringing it away. In extremely difficult cases it means giving the patient chloroform to lessen the bleeding, then cutting flaps of gum and the bone away sufficiently to release the roots (see Figs. 55 and 56 ).

We have shown that accidents may be frequently prevented by care in applying the principles of extraction, but, unfortunately, they are sometimes unavoidable. When they do occur. antiseptic treatment must be applied continually until definite signs of healing take place. Where a number of teeth are to be extracted in a septic mouth, preparatory antiseptic treatment 
should be carried out for a few days previously with peroxide of hydrogen, glycothymoline, Camphenol, or other suitable antiseptic mouth-washes. After the operation, peroxide of hydrogen must not be used, but the other mouth-washes should be continued. On the same day as the operation, the mouth-wash may be used hot every half-hour and kept in the mouth for a few minutes (see Fig. 57).

In very bad pyorrhœa cases where the lower teeth have to be extracted there is considerable risk of hæmatoma supervening if preparatory and after treatment is not attended to. Hæmatoma is an inflammatory condition caused by the locking in of blood similar to a contusion of bloodvessels. It may be a serious condition if the sockets are infected with micro-organisms

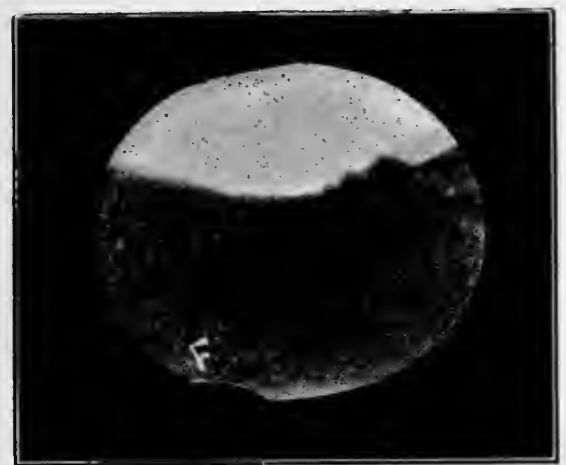

FIG. 55.-RAdiograph OF RIght Lower WISDOM ROOT BURIED WITHIN THE BONE.

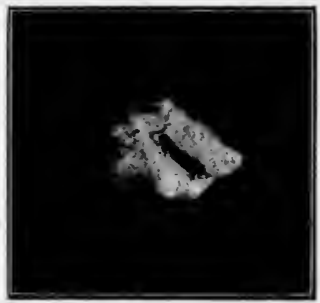

FIG. 56.-The BURIED WISDOM ROOT SHOWN IN FIG. 55.

from pus. The lower part of the patient's face appears bruised, and the surrounding edges are of a brownish-yellow colour. With the use of antiseptic mouth-washes and internal medicines the symptoms soon disappear, but the condition can be prevented by preparatory treatment. This condition, which the writer has seen several times after gas extractions, might easily bring local anæsthesia into discredit, which if used would probably complicate the condition, particularly if much adrenine is in the solution. The sockets of any of the teeth will occasionally give some trouble after extraction. If the patient is in a weak or debilitated condition, a socket, particularly a dry socket, or an indolent suppurating socket, may show no inclination to heal. 
These conditions must be watched and kept strictly aseptic by daily syringing and constant use of an antiseptic wash. Suppurating sockets will sometimes persist for some weeks, and must then be radically dealt with by swabbing out with 20 volumes peroxide of hydrogen, and followed by pure Bobœuf's Phénol Sodique or Camphenol. Explore with a ball-ended probe for loose fragments of débris or bone. When we are satisfied that it is clear, we can stimulate the socket with a trace of chloride of zinc, which is excellent for this purpose. When a socket is obviously septic it must be syringed at the time, especially in the lower jaw, with carbolic acid I in I20, Bobœuf's Phénol Sodique, one teaspoonful in a third of a tumbler of water, or Camphenol, $\frac{1}{2}$ per cent. solution in hot water. An annoying and sometimes a serious complication is caused by secondary hæmorrhage supervening. If a patient is known to bleed badly he should be prepared

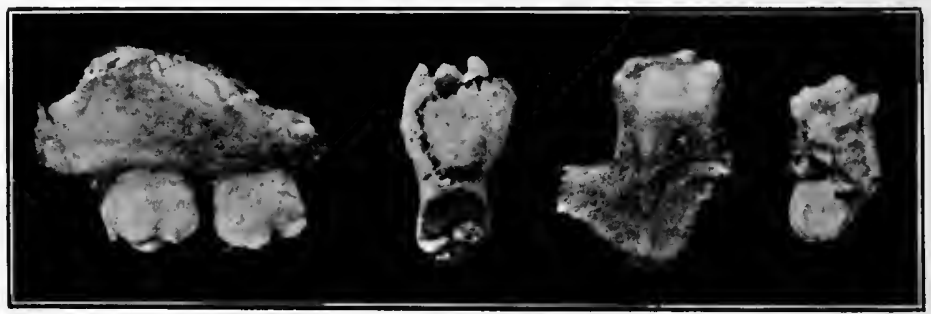

Fig. 57--Unavoidable Accidents in extracting Teeth.

by the administration of chloride of calcium, 5 to 15 grains in tabloid form, three times a day for a few days beforehand. The use of adrenalin tape or gauze as a styptic is effective if well packed into the socket. Some operators use one thing and some another, but secondary hæmorrhage cannot be temporized with. It must be definitely dealt with. It should be regarded as a leakage in the circulatory tubes, and must be stopped. If nature's provision of primary clotting fails we have to resort to assisting the process in an intelligent manner (see Chapter VII., p. I04). If plugs are applied they must be effectively kept in place. It is better to secure this at the first application, because the continual changing of plugs and the pressure used makes the gum tissues lose their contractile ability and become flabby. Sometimes digital pressure will cause contraction of the walls of the socket, especially if the outer wall of bone is flexible. Although 
the bleeding usually comes from the bloodvessels in the bony socket, yet the ability of the socket to hold the plug is important. The writer had a hæmorrhage case about eighteen years ago. The patient was a delicate anæmic girl about twenty-two years of age, with very dark complexion. Eight teeth in front in the lower jaw were extracted in the morning. About four o'clock the patient returned as the right lower lateral socket was oozing badly, and it was plugged. About half-past nine in the evening a message came that she was still losing a quantity of blood, and could the writer call at her house ? On arriving, the plug was removed and another put in, and after waiting half an hour there was no inclination to stop. The plug was removed, and the internal and external surfaces of the gum were pinched tightly for a quarter of an hour; at the end of that time bleeding stopped, and, after waiting for a further half an hour, did not recur. Instructions were given to the patient not to lie down in bed, but to recline. The bleeding was effectively stopped, and there was no further trouble.

The following method is an old and reliable one, as it provides a tightly fitting antiseptic plug with powerful styptic properties. The socket must be found from which the bleeding is coming. It must be cleaned out and a small plug of cotton-wool, saturated with carbolized resin and then dipped in calcined powdered alum, packed to the bottom of the socket, then another piece is placed on to that, and if necessary another piece. Count the pieces so that they may be accounted for afterwards. A pad of linen must then be put over it so that the bite keeps the whole thing in position, as the plugs must not move by the pressure of the blood. If necessary the lower jaw can be supported firmly by tying a four-tailed bandage over the head. If these instructions are faithfully carried out, the method fulfils the principle required. The carbolized resin is very sticky and appears to have a styptic effect in itself; the alum is a powerful astringent. After ten minutes or so the plug becomes hard and is antiseptic. Plugs of cotton-wool may be useless because the styptic gets washed away or diluted by the moisture, and also soon become foul. This method may be varied by putting in a plug of adrenalin gauze and covering it with the drugs mentioned above, and instead of the linen pad a thickly mixed piece of plaster of Paris in a mouldable state can quickly be put into place after effectively drying the gum. 


\section{CHAPTER XVII}

\section{THE CARE AND TREATMENT OF CHILDREN'S TEETH}

THE subject before us is one of paramount importance. In all ages the care and training of children in their physical and mental aspects has rightly demanded the attention of those who recognized that their capacities in adult life are the results in ratio of the guardianship of those who had the responsibilities of the upbringing or rearing of them. With the progress of modern medicine and hygiene much has resulted through a corresponding amount of attention bestowed upon children. On the other hand, there are discouraging factors which manifest themselves with the advance of greater knowledge with reference to hygiene. Generally speaking, each succeeding generation appears to be weaker in the natural resistance of the various tissues of their bodies. Eyes, limbs, hair, nerves, viscera, and teeth, seem to claim a disproportionate amount of curative treatment compared with that of former years.

The child as a patient means more to us than we, perhaps, have adequately realized. He is a powerful factor at the present time, and will be more so in the future, because so many bodily ailments and diseases in the community are proved to be caused by the carious or decayed condition of the teeth and morbid symptoms of the gums. However irksome the treatment of children's teeth may have appeared in the past, it is inevitable that every dental practitioner will have to take his part in the tremendous amount of work that will have to be done for these little patients. The question of dental treatment for children has been discussed for many years, and is now an important part of every dental practice. The man who tries to dodge it really stands to lose a great part of his clientele. Children are now educated at school in the principles of hygiene, which includes the personal care of the mouth and teeth. The consequence is that the children go home and describe what they have learnt 
at school; this in turn interests the parents, whose attention is thus arrested, and as the matter appears of importance it is doubly accentuated to them by the pertinacity of the children, who carry conviction right home to them in such a way as to suggest reproof that the matter is not common knowledge at home. The parents, again, always being alert for anything that is of importance to the welfare of their children, seriously proceed to become acquainted with the necessity of a tooth-brush, dentifrice, and antiseptic mouth-washes. This leads to anxiety if a cavity is discovered, and as this is a common occurrence the dentist must be found who can do it to their satisfaction, both with reference to its being properly done and without alarm to the child. If, after the child has been to be treated, the practitioner has not satisfactorily demonstrated his knowledge, the man will be found who does. In this way the parents and children are lost, which in many cases will mean whole families. The days have gone by for dentistry as a luxury; the practice of it is now a necessity, and has become popular instead of being such a dreaded speculative and coercive duty impelled by agonizing toothache.

\section{The First Principle consists of Tact.}

The first principle or object in dealing with children in the dental chair is the psychological control of the operator, or, in other words, tact on the part of the dentist in personally dealing with them. This is of far more importance than some men realize, but it is an inevitable factor under the force of circumstances which we have stated.

Children, as patients, whether of the doctor or dentist, have to be reckoned with, and the professional man who thinks to treat these little people as though they could be dealt with lightly, finds himself working on ground that, so far, he has not properly dug or trenched. Many a dentist has found himself totally unable to deal with a child simply because he has so thoroughly forgotten or overlooked the power that is concealed in the small person that he is wanting access to. The child must be estimated for, he must be tactfully yet properly handled. With it there must be conveyed to the little patient that alertness of judgment, complete kindness, and skill, such as a child can appreciate. Children are often susceptible of incompetence quicker than many adults, and should the operator once lose his 
purpose, his future control over the child, whether boy or girl, will probably never again be established. Children are sometimes more than masters, and are capable of dealing with an opportunity in a masterly way, and can completely frustrate all attempts to reconstruct anything that may be conceived with reference for further work for them. In short, we maintain that to be able to practise dentistry successfully upon children, one must at all times come down to their platform and ensconce oneself upon their level, for by so doing we have the power to see, as it were, through their eyes, the object which it is ours to attain. Once this principle is understood the quicker our object is secured. The dentist must be willing to see that his little patient is more or less a foe-which is the reflection of the patient's view of us-and must therefore be won. This must be accomplished with weapons suitable to their case and which, after all, are almost as imperative in dealing with our older patrons.

\section{The Psychological Power of Children.}

Upon entering a room children will often create a psychological profoundness which an adult often fails to command. There may be a definite expression of passive anticipation in their eyes. It remains for those whose duty or privilege it is to receive them to turn this anticipation to pleasure or disappointment. The child in being brought to the dental surgery has probably been mentally prepared by what he has been told, but it is up to the dentist to make the visit either a pleasure to himself or an ordeal for the child.

The compliment of being trusted by a child is no small responsibility, because children are wonderful readers of adult temperament, and it undoubtedly lies in the operator's power to render his services congenial, or otherwise, to his little patient by the mental attitude in which he carries out his work. If he is impatient he may as well not attempt to do any conservative work at all; but however irritable he may be in his own temperament, if he is fond of children he will be more successful than the most placid man who may happen to have no appreciation of a child's nature. Moreover, children seem in their entire unconsciousness of weakness to have a potency of personality which in many cases seems to be irrepressible. If you hurt or offend children they are capable of administering a sharp rebuke which is felt by the adult, not so much through their personal unconsciousness of 
weakness, as by the adult's absolute knowledge of their weakness. Children, moreover, are capable of showing extreme kindness and thoughtfulness for others; is it surprising, therefore, that those characteristics should be the levers whereby they can be controlled? They are living examples of doing unto others as they would be done by.

\section{The Levers of Control.}

Kindness, thoughtfulness, firmness, and technical knowledge, combined with manipulative skill, are the true factors that the child's dentist must be in possession of. Kindness in this case, however, must not be coupled with pity, because immediately we awaken any idea of pain or discomfort we have failed in our object. Thoughtfulness must not be a fussing around or a familiarity in dealing with them. Children are just as quick to resent this as adults, but if shown in one's manners towards them, it is exercised in the right place; and they don't wish it nearer the surface than that. Children also have a very keen sense of humour, and will hold you up to ridicule when out of your presence. They are also very clever mimics, and the dentist would rather think that respect and deference were at work in his favour rather than his endeavours for recommendation be lost.

\section{Types of Temperament.}

Some children require very little handling, while others will exercise every bit of ingenuity and tact that we can produce. There are a few well-marked types of children to be found at various times in the dental chair. There is the irritable and spoilt (the impossible) type. Then there are those that are " nervy," but highly intelligent. Again, there is the credulous child; care must be taken not to deceive them, as they are much better managed by being candid. There is also the child with a beautiful, radiant temperament, who will put up with pain and inconvenience in spite of any enquiry on the part of the operator, and who will rather apologize for any inconvenience which he may have unconsciously caused. It is a positive pleasure to work for such, and it imposes extra thoughtfulness upon us in order to avoid unnecessary pain or inconvenience to them. There is the enquiring child, who certainly helps, because his interest prevents him getting easily fatigued. Such children appear to derive much pleasure from what they see and hear. This sometimes 
has to be watched, otherwise accidents might occur through touching instruments and trying to manipulate appliances. There is also the deceitful child, who may go through the operation all right, and yet will exaggerate the pain or roughness which they assert you inflicted upon them. It is much better that the mother bring a child herself, for the first visit anyway, so that the nature of the operations may be explained, as a nurse is not often interested. It is not wise to let the mother interfere in any way after the work has commenced, as standing round the chair pitying or caressing would probably make the child succumb very soon to tears.

Many of our patients who are mothers will often mention some condition or other of their children's mouths to us, and refer often to one of very early years. Now it is useless to regard their questions as being irrelevant or trifling, because there is no period limit to our study and knowledge of dentition. Therefore, although it may not be practical to apply any treatment, yet we should be able to explain intelligently the symptoms to the mother, which she will either be satisfied with, or the reverse.

The subject of children's teeth may be dealt with as follows:

I. Caries and treatment.

2. Symptoms of normal and difficult dentition.

3. Irregularities, structural and positional.

4. The practical treatment of positional irregularities.

The cause and treatment of caries is dealt with in Chapters XVIII. and XIX., but we must modify the thoroughness of our preparation of cavities so as not to impose severity on our little patients between the ages, say, of three and a half to eight years. We have to temporize with them to a certain extent; as long as we can keep the teeth from pain we can do the work as thoroughly as the circumstances will permit. In a crown cavity with a continuous wall we can put a fairly good filling in, but temporary teeth have an unfortunate way of developing caries on their corners, which means somewhat deep cutting in order to secure proper retention. The large size of the pulps of these teeth precludes deep cutting, or we should expose them. If the cavity is deep, we should place a capping of eugenol and zinc oxide on a piece of card, or oxyphosphate of zinc mixed with equal parts of the liquid and eugenol.

Black oxyphosphate of copper cement makes a very useful 
filling for temporary teeth where there is insufficient hold, as it is very tenacious, sets quickly and very hard. We must keep it dry with a napkin or cotton-wool rolls. "Sanoid" cramp wool rolls are very useful for children's mouths. Copper amalgam is a good material, as the copper salts that become active after it has set preserve the dentine, but its action has the objectionable feature of discolouring the tooth tissue, which makes its use inadvisable in permanent teeth. For superficial saucer-shaped cavities and hypersensitive surfaces, caused by fracture, the careful application of nitrate of silver is useful and arrests caries efficiently, but turns the tooth tissue black where it is applied.

It is advisable to avoid extraction if possible in the case of temporary teeth and defer it until the permanent teeth loosen them. If the temporary molars are extracted early, the six-year molars will probably move forward and crowd the bicuspids badly, either lingually or buccally. If there is pain and the child is kept awake at night with a carious tooth and we consider it to be wise to retain it, a good anodyne remedy is the topical application of a mixture of equal parts of liquid carbolic acid and glycerine. The bottle must be shaken before using, as the two ingredients separate on standing. The parent should be instructed to dry the gum and cavity with a piece of clean linen, and then with a tiny camel's hair brush to paint one drop only of the mixture into the cavity and around the gum. It may then be applied once again at the end of an hour, but not again that night. It may be applied in the same way during the next night. The efficacy of this remedy is secured by its continued treatment rather than by one application. A hot raisin covered with a small piece of linen and placed between the tooth and cheek will often give relief in cases of threatened abscess. When extraction of a tooth is absolutely necessary, apply the calorific anæsthetic mentioned in p. $20 \mathrm{I}$.

Root treatment and filling are not practicable with temporary teeth, and must not be resorted to in the case of a permanent tooth if the roots have not been completed and its apical ends are open.

The main difficulties in preparing cavities in children's teeth are that the mouth is tiny; the gums are shallow, and do not retain wool rolls well; the saliva is copious, especially when a suggestion of pain presents itself, whereupon the salivary glands are 
stimulated; dentition is active and there is extreme sensitiveness of the teeth generally; the pulps of the temporary teeth are very
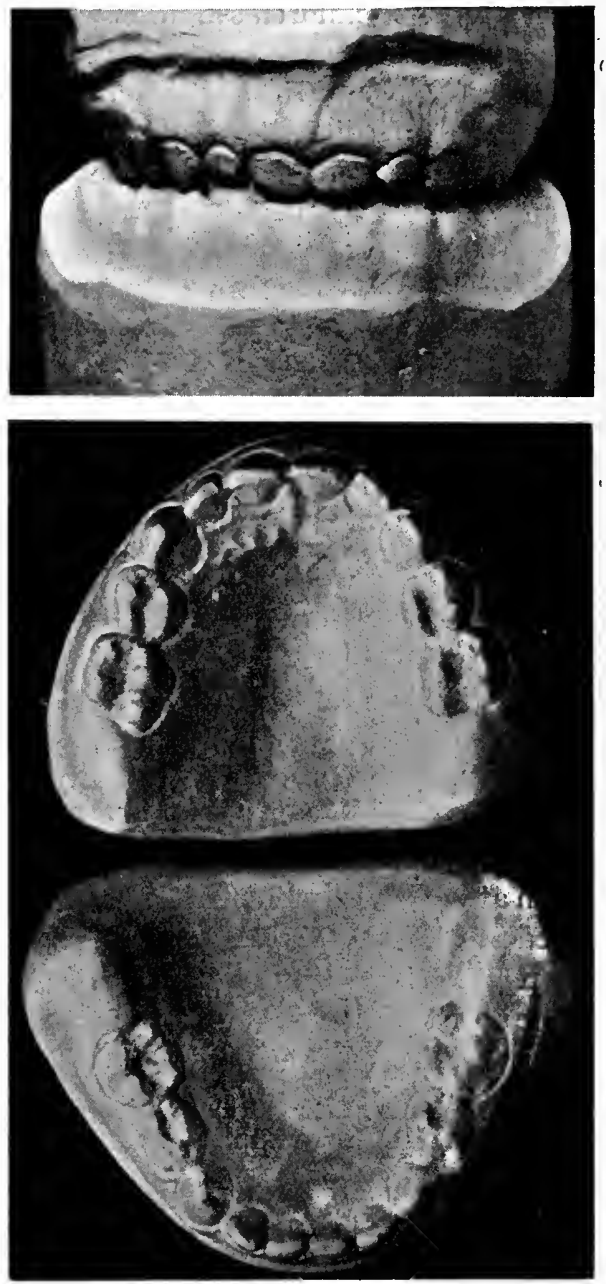

Fig. 58.-Models of a ChIld's Mouth, Aged Five Years, showing the Deciduous Set of Temporary Teeth, which are Normal in EVERY RESPECT.

large in relation to the teeth when compared with the permanent ones; the little patient's mouth soon gets tired and keeps closing; the instruments available are not as convenient as they might 

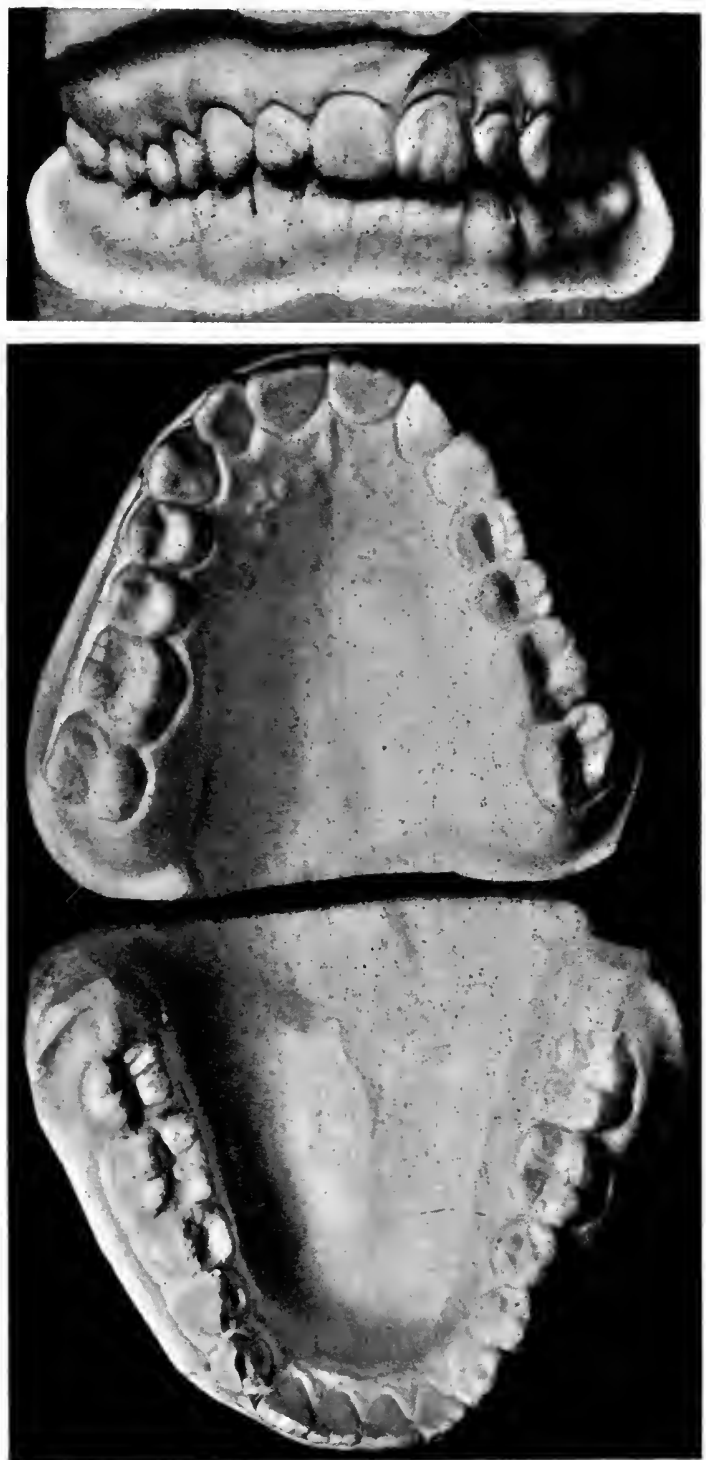

Fig. 59.-Showing Models of the Same Child's Mouth at the Age of Fourteen Years (See Fig. 58).

The only evidence of the mouth belonging to the same child is the markings of the rugæ. These are identical, but are much larger and distributed farther apart. 


\section{DESCRIPTION OF FIG. 60.}

In order to understand clearly where we wish to move a tooth to its desired position, we must fully realize the action of the device we decide to employ.

$A$ is a retracting bar which can be used as a spring mounted on a vulcanite plate; or, with a carrying tube soldered on to a crown band, with a screw thread and a nut, to cause retraction.

$\mathrm{B}$ is a ball-headed screw for extending the tooth forward. It is made by cutting a piece from a length of screw threaded wire, which is supplied by the dental depots, and is made from an alloy called " Platinoid." One end is heated and borax is applied to it, but only where the solder is required. A suitable piece of gold solder is then fused on to it while in an upright position which thus allows the ball to form. To mount it into its place we take a fine drill or a bur and drill through the vulcanite, which has been thickened, at a right angle. The hole is then enlarged, if necessary, with a cross-cut fissure bur a size smaller than the screw. Another piece of screw is then taken and filed to a square point, which is then warmed, and a tap is made which receives the screw.

C. Another way of getting the thread is to purchase a threaded tube to fit the screw and vulcanize it into place. These threaded tubes have been used in the specimen for figure.

$\mathrm{D}$ is a retracting bar mounted on to a vulcanite or gold cap, $\mathrm{H}$.

E, Coffin's expansion spring; the ends are well embedded into a vulcanite plate, which is sawn through the median line after completion and slightly expanded once a week.

F. Read's expansion screw is very convenient and useful. The screw and tube must be fixed in a level position, the ends $I$ and 2 allow the plaster to hold it in place while packing.

G. A vulcanite capped plate with front retracting bar. 


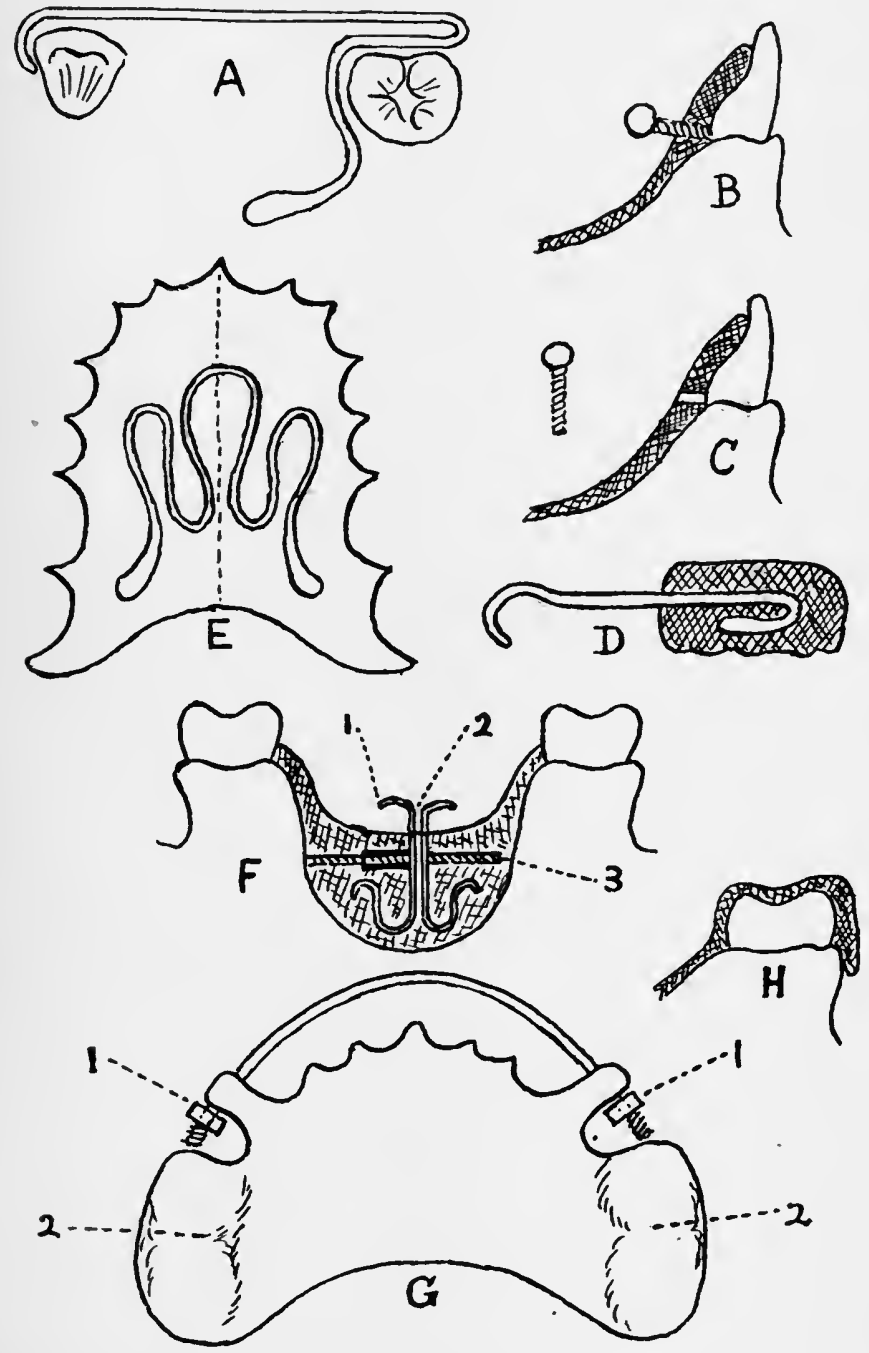

Fig. 60.-Diagram showing Devices employed for Retraction. Extension, AND EXPANSION. 
DESCRIPTION OF FIGS. 61-66.

Fig. 6I.-Shows a Specimen ivith Natural. Teeth mounted in Modelling Composition to Demonstrate the Common Forms of IRREGULARITIES.

The following figures show the various devices applied for their regulation:

Fig. 62.-Shows a Palatal View of the Upper Teeth, demonstrating the Application of Angle's Molar Crown Bands with RetractING BAR IN POSITION.

Fig. 63.-Shows Read's Expansion Screw Plate in Position. It has been expanded with Tree Turns of the Screw to give a View of the Split Plate.

Fig. 64.-Shows the Lingual View of the Lower Teeth.

Fig. 65.-Shows a Vulcanite Plate embodying Retraction Bars for Central and Canine Teeth mounted in Vulcanite Caps for SEATING.

Ball-headed screws placed in position against the laterals; a crown band is placed on the central with a hook and another hook on the vulcanite plate. A rubber band is fixed on these hooks, which has to be removed before the plate can be taken out for cleaning. The central tooth shown in the corner, demonstrates how a tooth can be moved and the root bent if pressure is applied to an undeveloped root.

Fig. 66.-Shows a Vulcanite Plate with Retracting Bars bent so as to Gain Length of Spring Necessary For Retraction.

Ball-headed screws in position at the angle necessary for moving the teeth into the desired position. These screws are mounted into threaded tubes, which are vulcanized in.

A careful study of the various types of irregularity embodied here, and the devices applied for their removal, with the considered extraction of teeth to secure ample room, will enable the student to pick out any point that he wishes to apply to any case in practice. 
FIG. 61 .
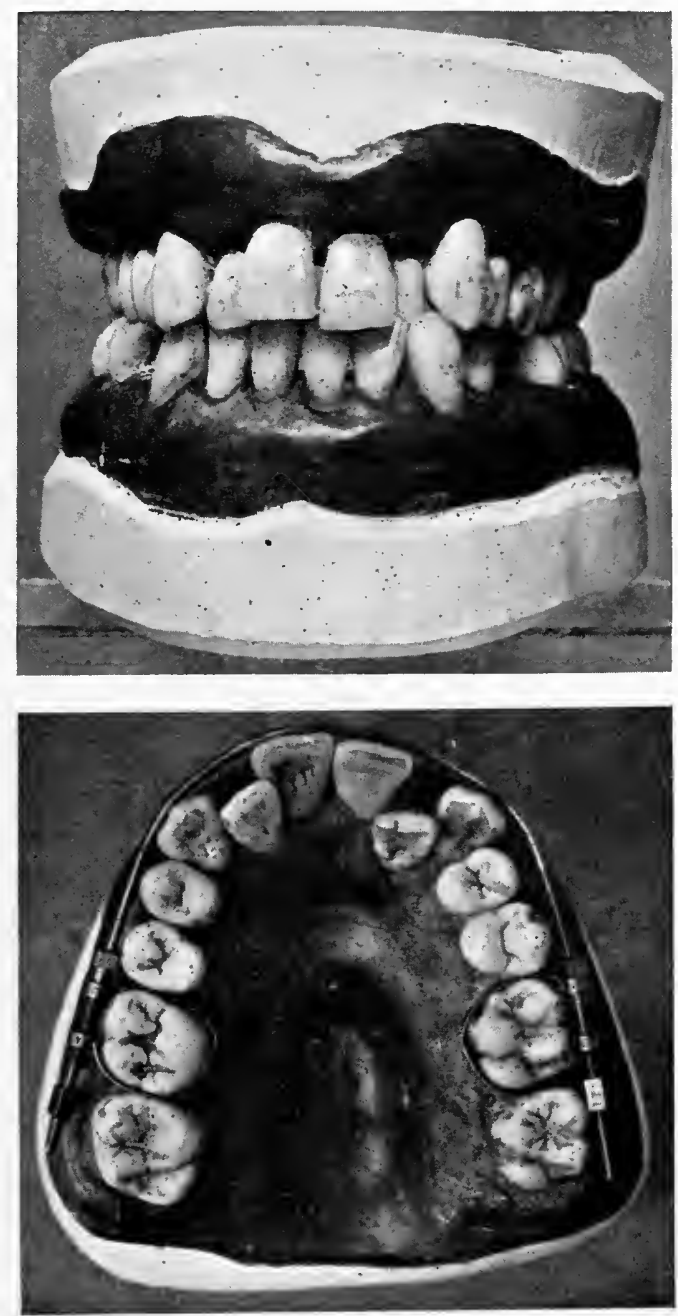

FIG. 62. 
FIG. 63.
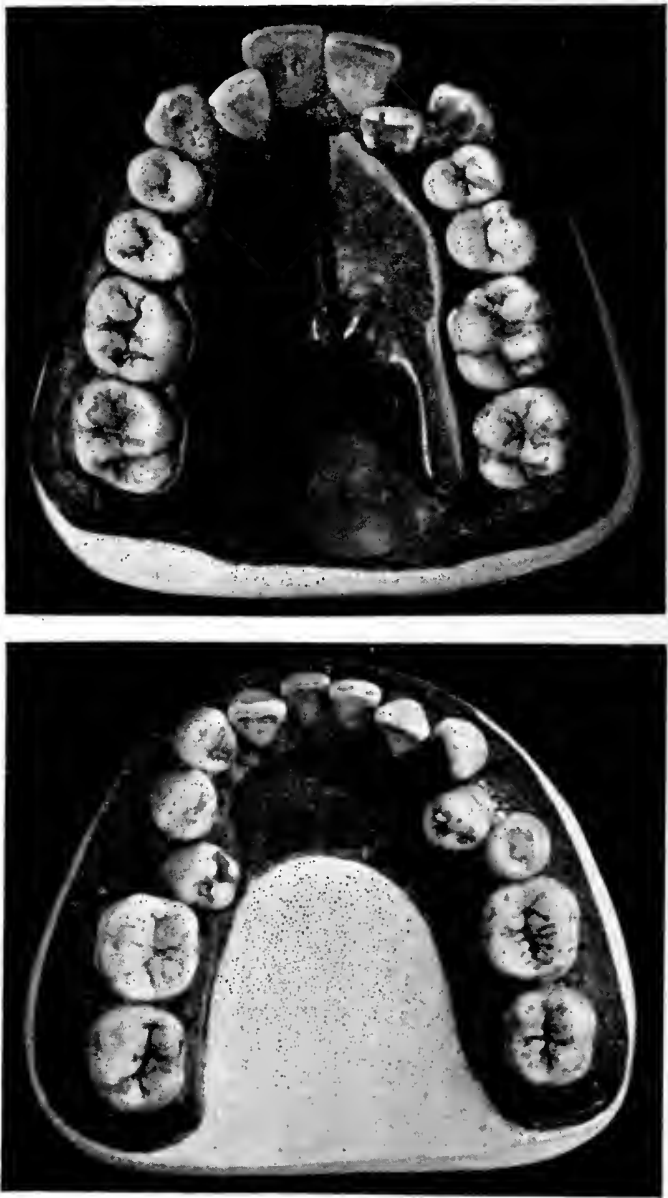

FIG. 64. 
THE CARE AND TREATMENT OF CHILDREN'S TEETH 247

FIG. 65 .
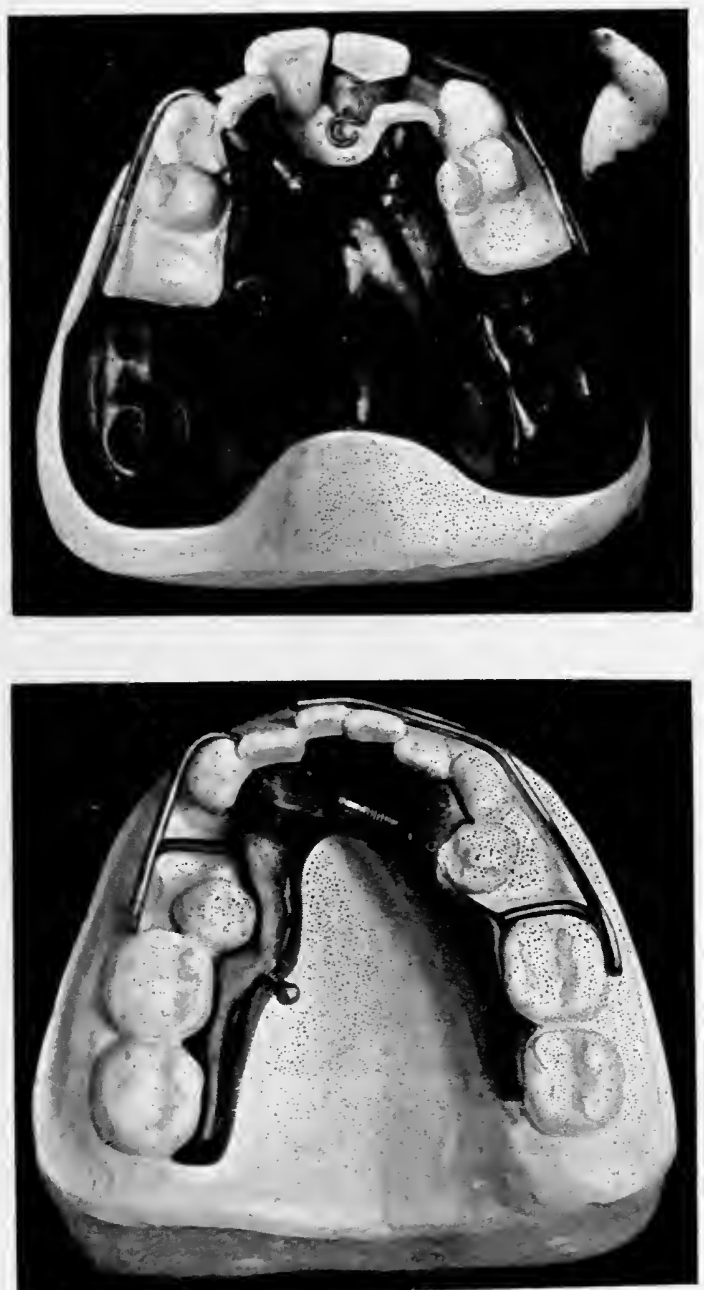

FIG. 66. 
be, which would profitably occupy the investigation of a com: mittee of operators who would co-operate with the manufacturers in order to produce some convenient appliances.

The ages of the various stages of the development of the teeth both in fœetal life and infancy have been described in our earlier chapters, but a great deal of interesting study can be applied to the details of the development of the teeth and jaws from birth to mature completion. Many authorities have carefully and comprehensively described the subject, which is well worthy of attention, with the result that the methods which we employ in practical work are rendered clearer in their application and results. An important feature is the elongation of the jaws to accommodate the permanent teeth. In many cases there appears to be an absence of relation of the sizes of the teeth to the length of the jaws. There is such an abundant variation in the sizes of teeth that they cannot be satisfactorily classified, but there is constant evidence that the jaws do not always develop to the size necessary to accommodate the teeth. The cause of this incongruity must either be in the activity of the cells of the dental follicles or those which should develop the dental portion of the jaws (see Figs. 58 and 59 ).

Another important point is the nature of the substance of the bone which constitutes the walls of the crypts, and the alveoli which contain the teeth in varying stages of formation. The bone is remarkably tough and elastic. In dry specimens of infants' skulls we find the soft cellular portions of the teeth have disappeared, and portions of completed calcified structure remain. These calcified parts consist of the cusps, and later on, according to their age, we find larger portions and then the completed crown; later still, the roots are found in varying stages of completion up to final eruption and position in the dental arch. In the extraction of children's teeth great care has to be exercised in order to avoid disturbing the permanent dental follicles and injuring them, or even rupturing them. The contents of a permanent follicle may be brought away with the extraction of a temporary molar; fortunately this accident does not often occur. Care must also be exercised in extracting, to avoid dislodging permanent teeth which are well through the gum but have only about a quarter of the length of their roots formed. The periods of dentition, given on p. 36 , should be familiar to us, so that our minds can always visualize the con- 
ditions and form of the interior of the jaws at the various ages involving our operations.

The symptoms which accompany teething are usually: the discomfort of the infant, as shown by his distress when crying out with pain; a general lack of tone in his physical appearance; cheeks red or flushed; tendency to bite everything; increased flow of saliva; gums appear full and tight, and the mouth hot. When dentition is not proceeding in a normal manner, it causes much disturbance locally, and also in the general system. The local symptoms may be acute inflammation of the gums at the seat of the erupting tooth, and also ulceration which may extend over the gums; this condition is called "odontitis infantum." General disturbance of the system may be indicated by great restlessness, convulsions, and even paralysis in some cases. The child cuts his teeth accompanied either by diarrhœa, constipation, and gastric disturbance, or with cough and bronchitis, eruptions of the skin of varying intensity, and disturbance of the genito-urinary organs. The treatment of these conditions is usually dealt with by a medical man, but sometimes relief can be given by the dentist in lancing the gums where tension discloses the position of the cusps of the tooth, and also the application of an astringent to the gums.

Irregularities of the teeth are a source of disfigurement and an embarrassment to efficient mastication. They may be either structural or positional. In the former case we find hypoplastic teeth; they have pits and grooves on their coronal surface caused by an arrest of deposition of lime salts from the blood during the development of their crowns; syphilitic teeth, which are conical with deep ribs across the crowns; geminated teeth, and those with enamel nodules upon their crowns. Also supernumerary teeth (see Fig. I2).

Positional irregularities are those caused by the wrong position of the teeth in the dental arch. They may be classified as:

I. Superior protrusion, where the upper front teeth are very prominent.

2. Inferior protrusion, where the lower front teeth are in front of the upper ones.

3. Open bite in front caused by closure of back teeth.

4. Contraction of the dental arch usually in the region of the bicuspids. 
5. Internal and external crowding of any of the individual teeth in relation to the arch and the normal articulation.

The practical treatment of positional irregularities of children's teeth is termed " orthodontia " or " regulation."

The objects which we have in view when regulating teeth are:

I. The artistic consideration of the patient's facial appearance.

2. The provision of a more efficient mastication.

3. The preservation of the teeth by making facilities for the proper cleansing of the interdental spaces.

These three objects provide us with some definite aim by which to proceed. They are all important, but the second one is the main consideration and must receive adequate attention.

The tendency of all irregularity is towards the median line. If the temporary molars are extracted early, the six-year molars will move appreciably forward and cause crowding of the bicuspids when the time for their eruption arrives.

The main causes of irregularity are either congenital or acquired.

Congenital irregularities may be caused by lack of proportion of the lower jaw to the upper one; vaulting of the palate; elongated lower jaw; also heredity.

Acquired irregularities may be brought about by the premature extraction of temporary teeth; the retention of obstinate temporary ones; injudicious extraction of permanent teeth; bad habits, such as thumb-sucking and mouth-breathing. Adenoids are responsible for many irregularities, as the constant struggle to maintain free breathing has a powerful constricting action on the bones of the face, and particularly the superior maxillæ, with the result that there is a strong tendency to superior protrusion and contraction of the arch.

The surgical principles of regulation are-

I. Do not attempt to apply pressure to a tooth until you have secured definite and ample space to push or pull the tooth into. This is a very important matter, and has always caused a deal of controversy. It is quite obvious that we cannot push or pull a tooth into its place without room to do so, but this is not sufficient. We hear and also read of instructions for regulating teeth, and the inadvisability of extracting a tooth even for this purpose. Expansion of the arch is an efficient means undoubtedly for providing room, but if it means just getting the irregular teeth into position, we maintain emphatically that it is not sufficient. 
There is one thing missing in dental literature upon this point, which is, the record of these crowded regulation cases. We have observed again and again that, in practice, teeth revert to irregularity again if ample room has not been provided. There should be consideration for the septum of bone, which must be thick and strong enough to hold the teeth, and due allowance must be made for the position of the wisdom teeth.

2. The favourable age for moving teeth without disturbing dentition unduly is from twelve to fourteen years, and not after sixteen or seventeen years for completion. We must be careful to know what strain we may be putting upon the soft uncompleted root of a tooth, as we may move the crown and unfortunately bend the root (see Fig. 65).

3. Hypertrophy of the gum will occur temporarily, especially if the teeth are moved too quickly, but is harmless and can be controlled if the pressure is either gently kept up, or, if stopped, the tooth must be held rigidly in place, when absorption will take place. The teeth must not be allowed free play, as the results may be serious to the sockets, which may be permanently enlarged.

4. When teeth have been brought to their assigned positions and the pressure stopped, they must be accurately held in position for twelve to eighteen months, to allow for the consolidation of the bone around the roots, otherwise the teeth will move towards their original positions.

5. Be careful to avoid moving teeth too rapidly, as it may set up inflammatory trouble through strangulation of the apical vessels of the roots, rupturing the periodontal membrane, or separating the suture of the palatal portion of the superior maxilla.

6 . Strict cleanliness must be carried out to keep the tissues free from irritation and inflammation, also the fermentation of mucus and food may provide sufficient concentrated lactic acid to discolour and partially disintegrate the surface of the enamel of the teeth, especially where caps on plates and also collars are used. It is very disappointing to the child's parents and exasperating to the operator to find, when the case is completed, that there are enamel markings which cannot be effaced.

The subject of "orthodontia," or regulation of children's teeth, is such an important and wide subject that to the inexperienced practitioner it appears bewildering and much too 
complicated. The display of elaborate appliances for the work rather has the tendency to convince him that it belongs to the orthodontic specialist. However, in a general family practice, useful work can be done by any man who has good mechanical intuition. The following mechanical principles or devices applied either upon a denture or tooth crown bands will be found to be effective in most cases of irregularity:

(a) Simple extraction. This means that by the timely removal either of a temporary or a permanent tooth the objects of regulation may be secured without further interference; or it may be carried out in order to provide ample room for moving another tooth into its place. If we take the case of an externally crowded upper canine, the first thing to be considered is the extraction of the lateral or the first bicuspid if room cannot be provided for bringing it into line. The objection to this procedure is that when the canine is brought into its place, the sloping or inclination of it produces an ugly triangular space at the cervical surface. The better way is to extract the second bicuspid, pull the first bicuspid back, and then the canine; in this way the inclination is distributed between two teeth and the space is not seen (see Figs. 79 to 82 ).

(b) Rubber rings and hooks. Rubber has a powerful pulling action, and is capable of setting up trouble if not kept within control. A rubber ring placed loosely around a tooth will work its way up the face of the root under the gum, causing much soreness and consequent inflammation. Too powerful a band will have a similar effect even if it is fixed so that it does not work under the gum. A metal collar must be fixed on the tooth to be moved with a hook soldered upon it. In order to move it, another hook is placed either on to a plate or to another crown band (see Fig. 65).

(c) Expanding the arch, using Dr. Coffin's expansion spring, Read's expansion screw or Angle's appliances (see Figs. 60 and 63).

(d) Ball-headed screws or hickory pegs. Hickory wood is supplied in round sticks, and is used for wedging a tooth forward. A vulcanite plate is made and thickened against the tooth to be moved. A suitable-sized hole is drilled into the vulcanite, the hickory stick is thinned down at one end and pushed well into the hole, the inside end is cut flush, and the part which goes against the tooth is left with an appreciable piece standing outside. When the wood gets wet in the mouth the wood splays 
out and swells, thus developing a wedging pressure on to the tooth. The pegs have to be constantly renewed. A better and quicker method is the use of ball-headed screws. They are easily fitted on to a vulcanite plate, and move a tooth with precision, quickness, and to any distance that may be required (see Figs. 65 and 66).

The screw is given a half-turn twice a week until the tooth is in position. If it has to be pushed half-an-inch, it is well to commence with a medium length, and place a longer one in when it has reached the extent of its capacity.

(e) Cap plates. Gold or vulcanite caps are fitted over the molar region, which raises the bite. They should be made on models mounted in an articulator and the biting surface adapted for the patient's comfort as far as possible. The inside, which fits the teeth in the mouth, must be kept scrupulously clean, or the acid fermentation from mucus and food will attack the enamel of the cusps (see Figs. 60 and 65 ).

( $f$ ) Springs and wires. These must be made of suitable thickness and well embedded. We have an analogy here with the insertion and attachment of a muscle. The muscle is well seated so as to be unaffected in the pressure of expansion and contraction, which is employed to move the part that is acted upon (see Figs. 60, 7 to 76$)$.

$(g)$ Jack screws, crowns, crown bands, bars for retracting and expanding, screws and nuts (see Fig. 62). These constitute the main features of the American system. Jack screws may be used as they are for expansion, but they are very powerful, and care must be taken to gently apply pressure, or the tooth that is being moved may fracture, especially if it is a lateral. If used alone, the jack screw is ligatured securely into place or it may be mounted on a plate.

(h) Retaining bars and plates (see Figs. 62 and 76 ). We have prepared a series of illustrations, which appear on the following pages, to enable the student to grasp the principles which we have described in this chapter. A careful study of them with the descriptive notes appended will demonstrate the application and use of the main mechanical devices used for regulation of children's teeth. 

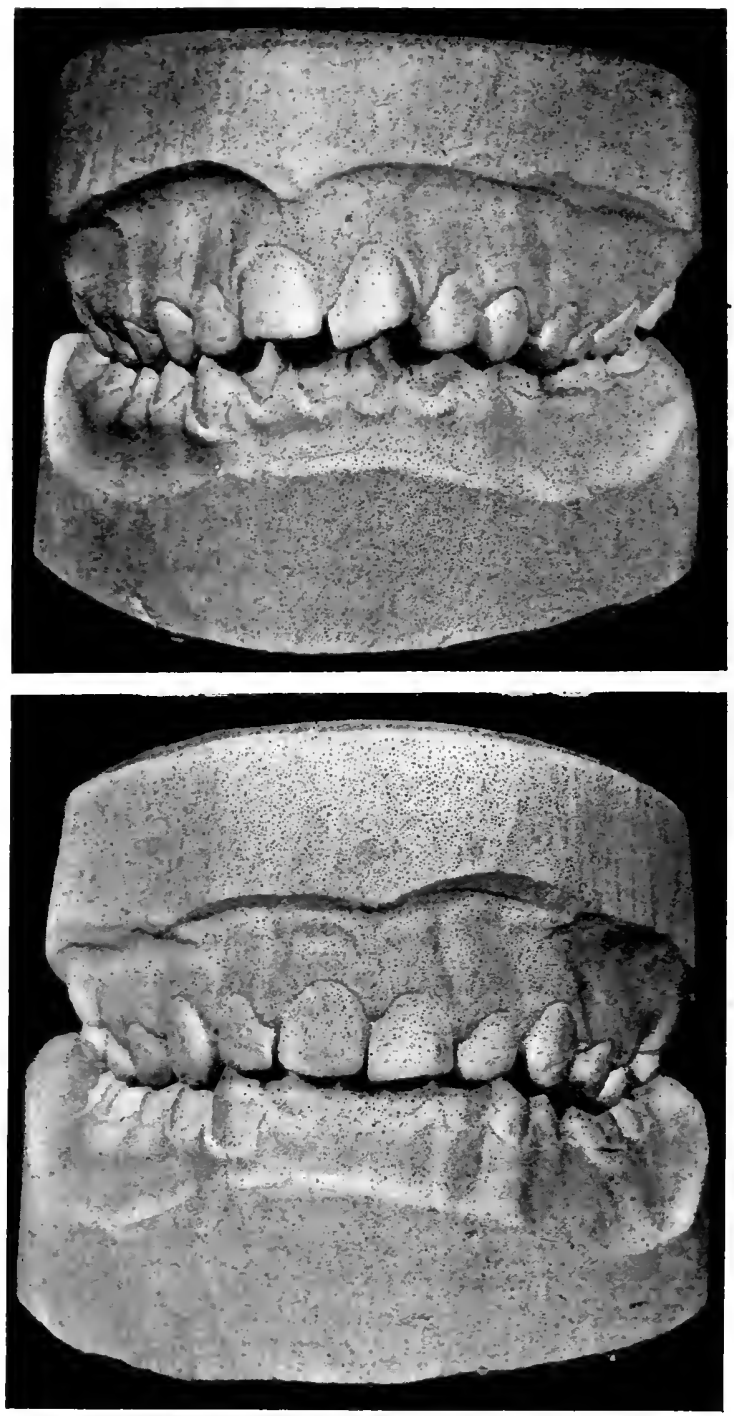

Fig. 67.- Shows a Case of Superior Protrusion in a Boy Aged Thirteen.

Fig. 7.3 indicates the extent of the protrusion.

Fig. 68.-Shows the Case when it was completed, Twelve Months AFTER.

The same models are shown in Fig. 76 with the retaining plate in position. 

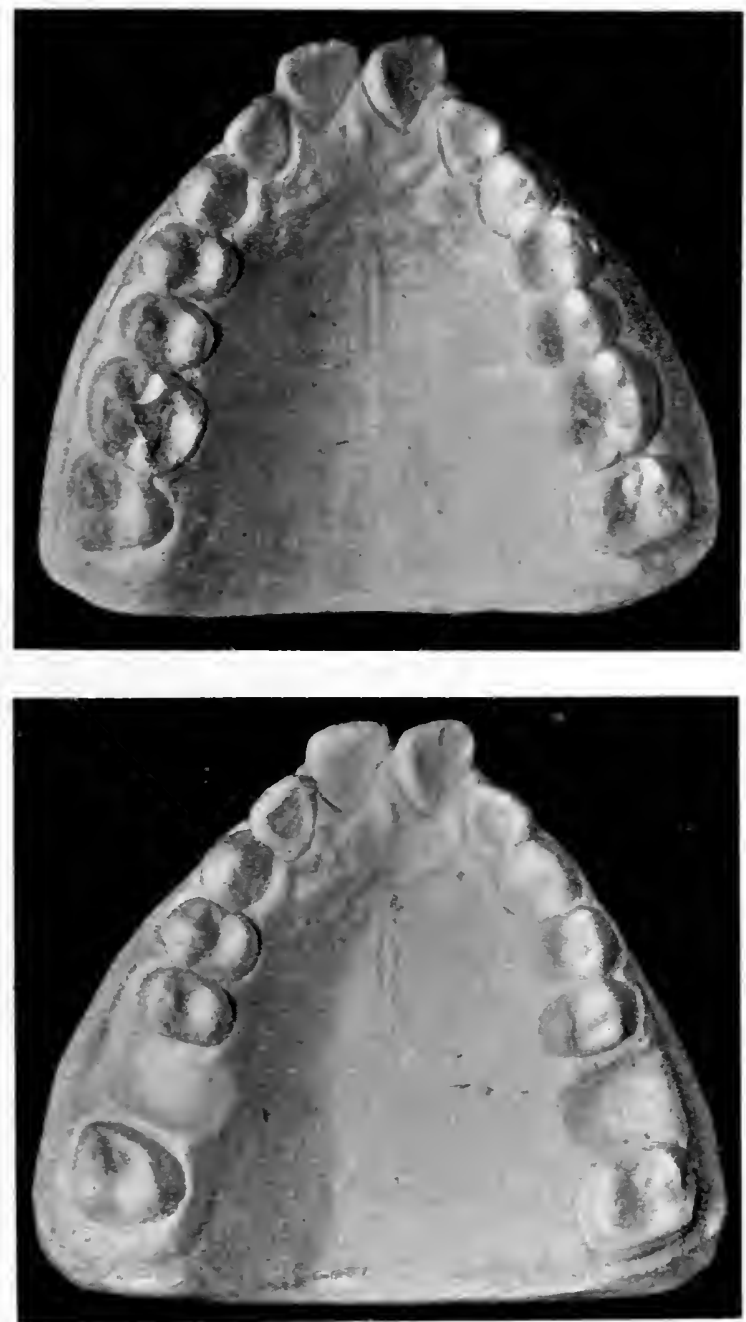

Fig. 69.--Shows the Palatal View of the Case before Anything WAS DONE.

The six-year-old molars are badly decayed. We decided to extract them.

Fig. 70.-Shows the Palatal View with the Molars taken out. This resolves the case into retraction of the ten anterior teeth. 

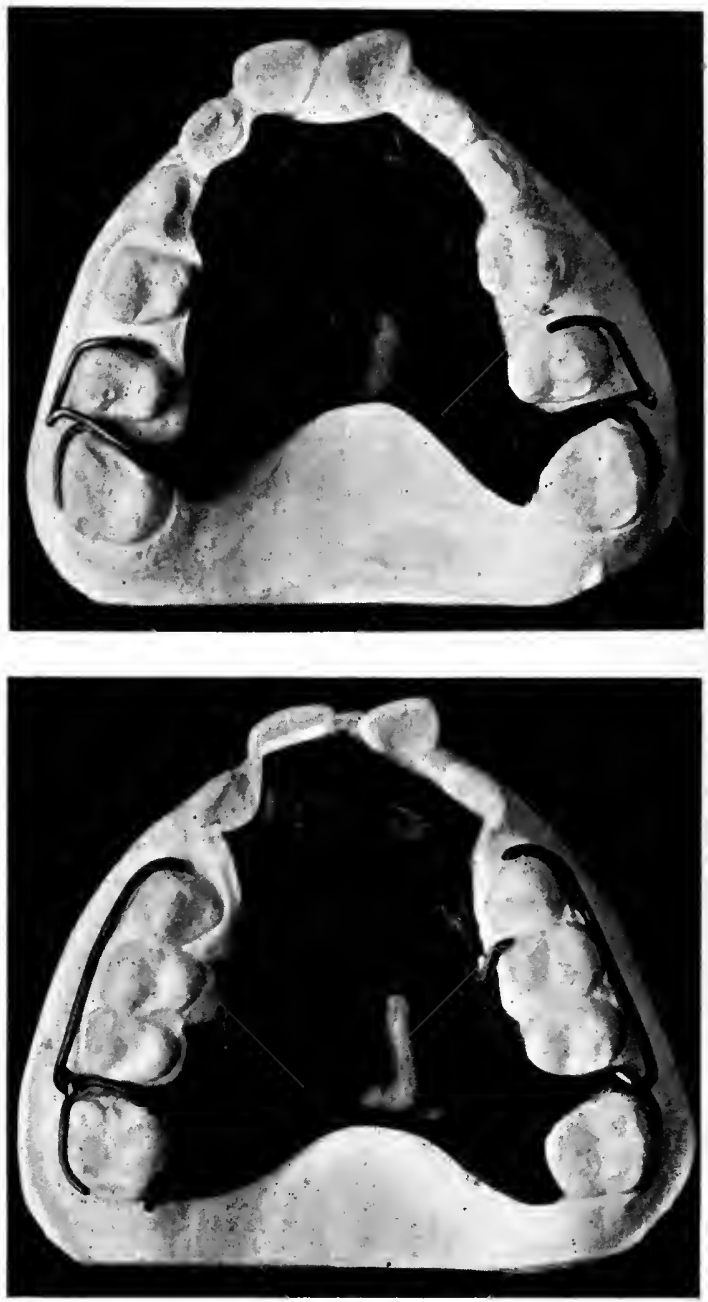

Fig. 7I.-Shows the Plate that was used to Retract the Second Bicuspids, with a Thick Raised Block to open the Bite while the Back Teeth moved Upwards in their Sockets to Fix the Bite so that Room was secured to Retract the InCISORS. (No. I Plate.)

Fig. 72.-Shows Plate No. 2, which moved the First Bicuspids and THE Canines. 

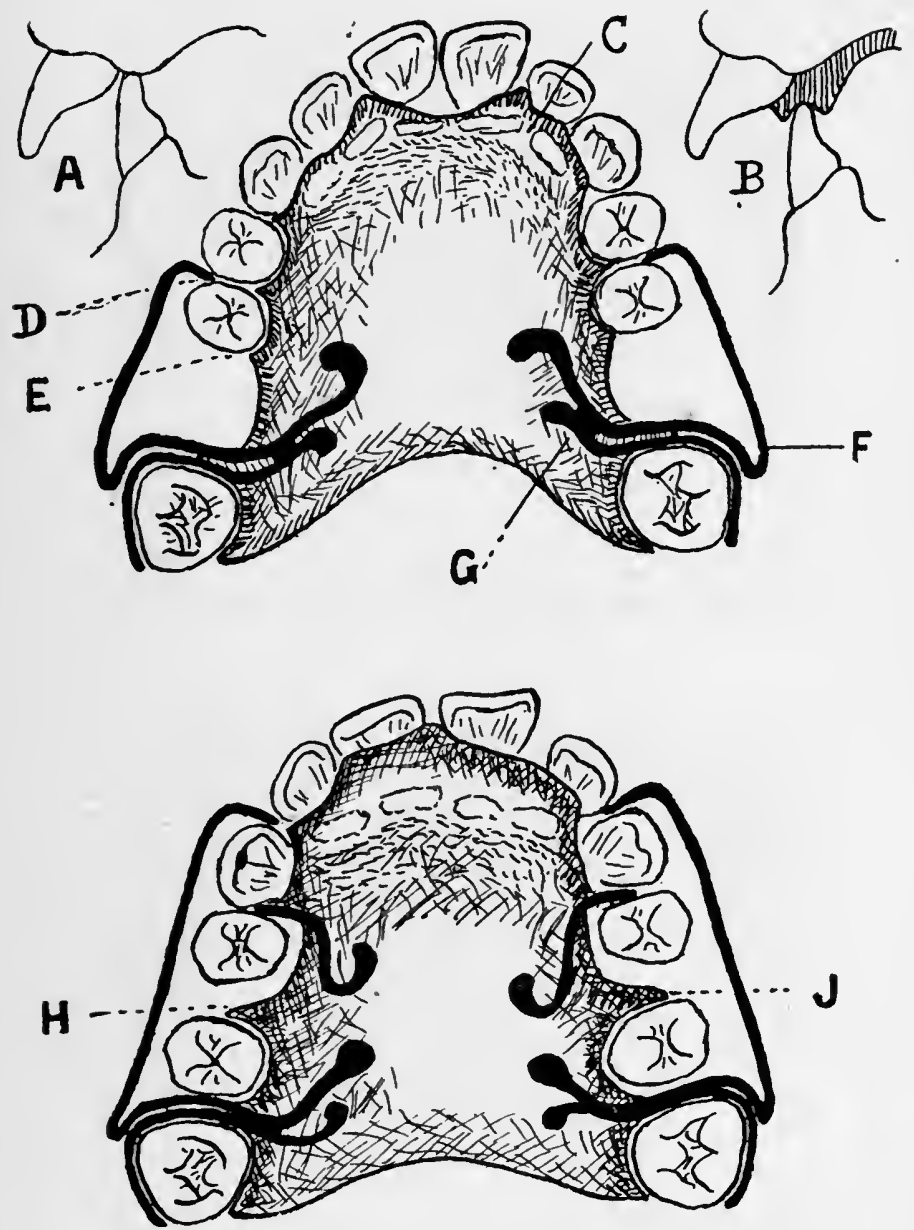

Fig. 73.- Silows a Diagram of No. i Plate before its Action was APPLIED.

A. bite before opening it; B. bite opened by vulcanite block; D, applied end of retracting bar; $F$, bend of bar to secure length of spring for action; $\mathrm{E}$, shows that the vulcanite must be removed to allow for tooth being moved backwards.

Fig. 74. - Shows a Diagram of No. 2 Plate before Action was APPLIED.

$\mathrm{H}$ and $\mathrm{J}$ show vulcanite stops to retain the second bicuspids while the other four teeth were being moved into position against them. 

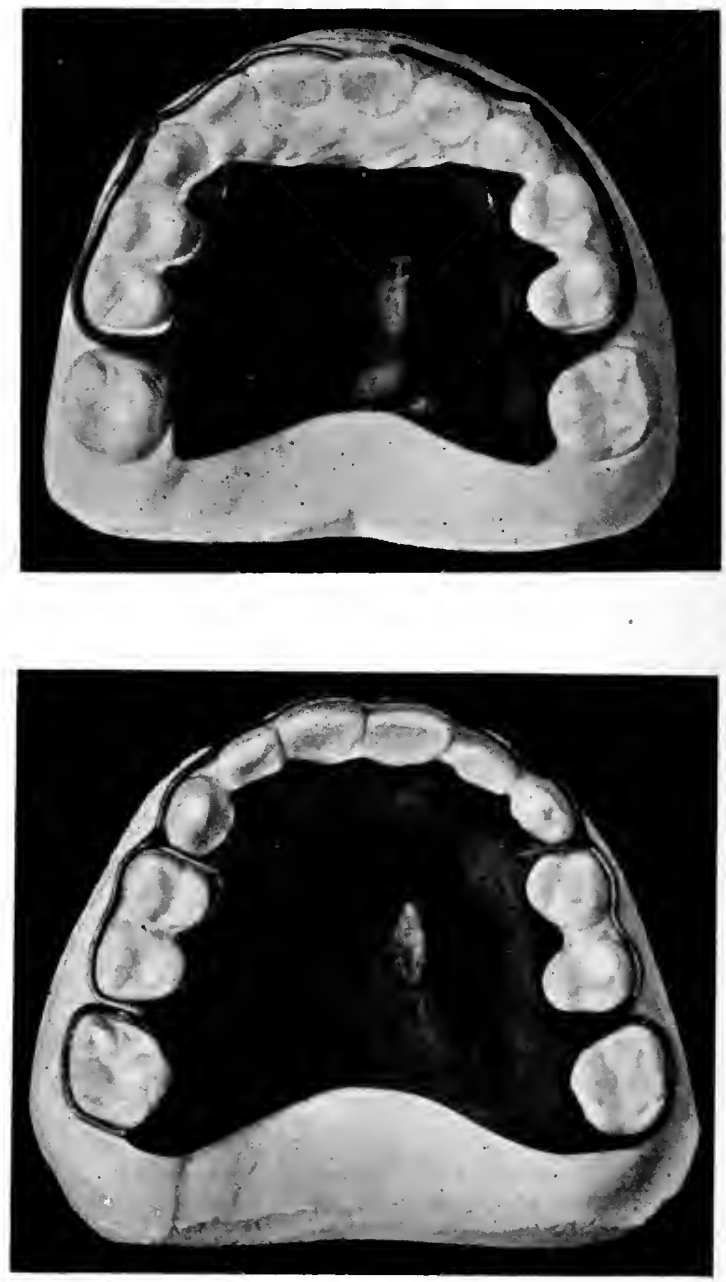

Fig. 75.-Shows No. 3. Plate, which moved the Incisors into Position.

Fig. 76.- Shows No. 4 Plate, which is a Duplicate of the Retaining Plate. 

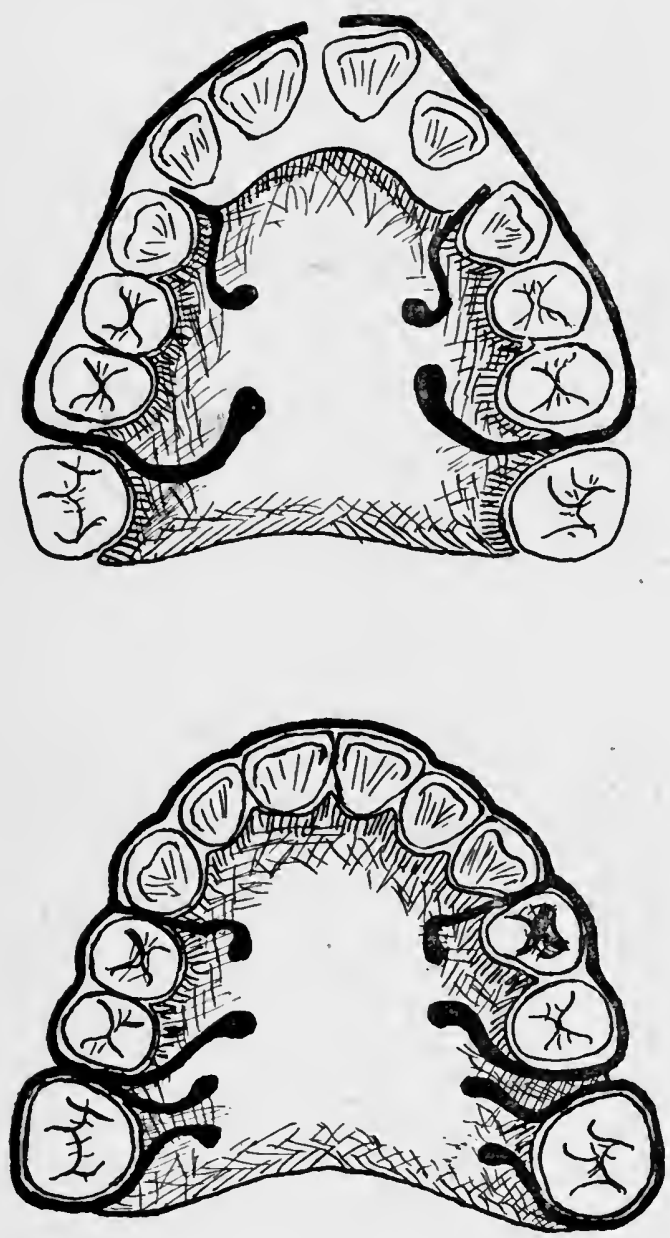

Fig. 77.-Shows a Diagram of No. 3 Plate before action was applied.

The canines and bicuspids have a retaining stop to hold them while the incisors are being moved. The vulcanite against them had to be generously cut away to accommodate the swollen gum, which the new shape of the anterior portion of the palate accentuated.

Fig. 78.-Shows the Construction of the Retaining Plate. 

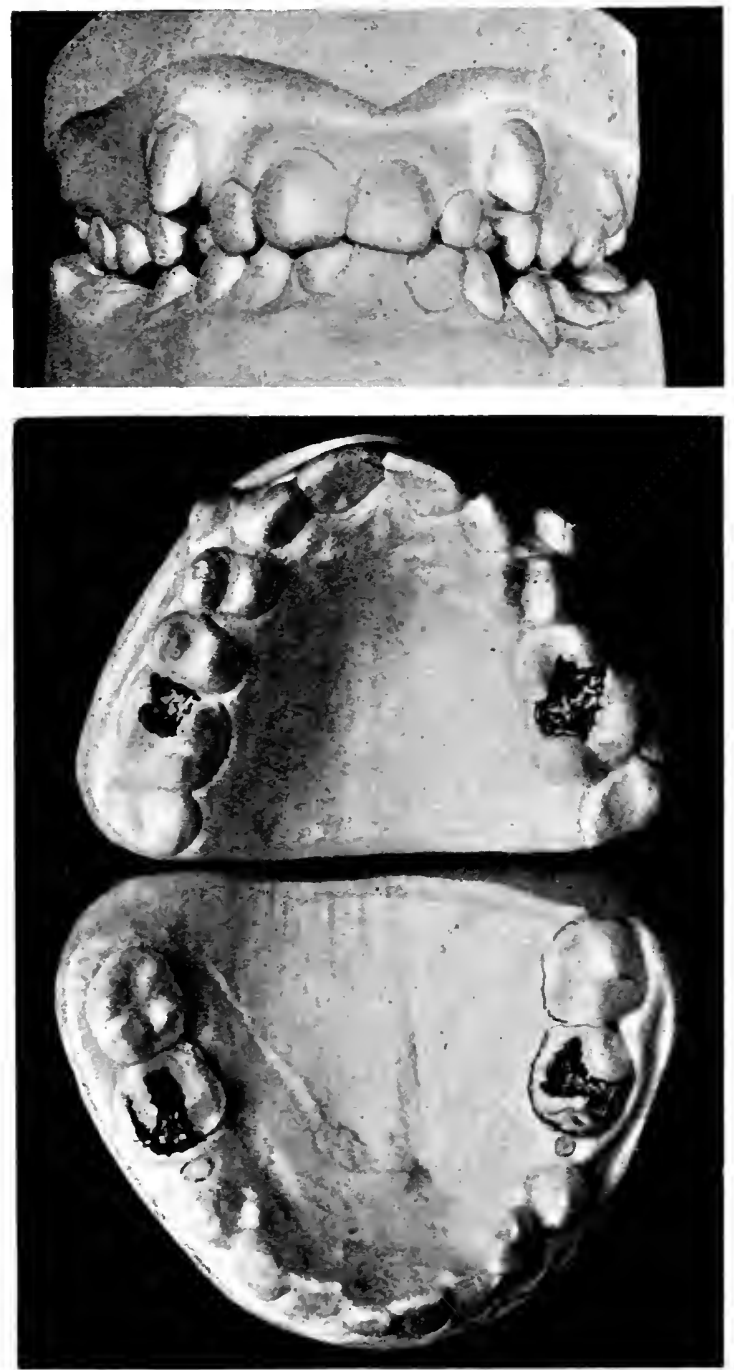

Fig. 79.-Shows an Extreme Case of External Crowding of the UPPER CaNinies.

The right molir was hadly decayed; also the left second bicuspid and sixyear molar. We decided to extract the right molar and the left second bicuspid. The left molar was filled with amalgam. On the right side we retracted the two bicuspids. On the left side the first bicuspid was retracted. This operation thus secured ample room for bringing the canines into line with the arch. Two six-year molars were extracted in the lower.

FIG. 80.-Shows Palatal and Lingual View of the Case. 

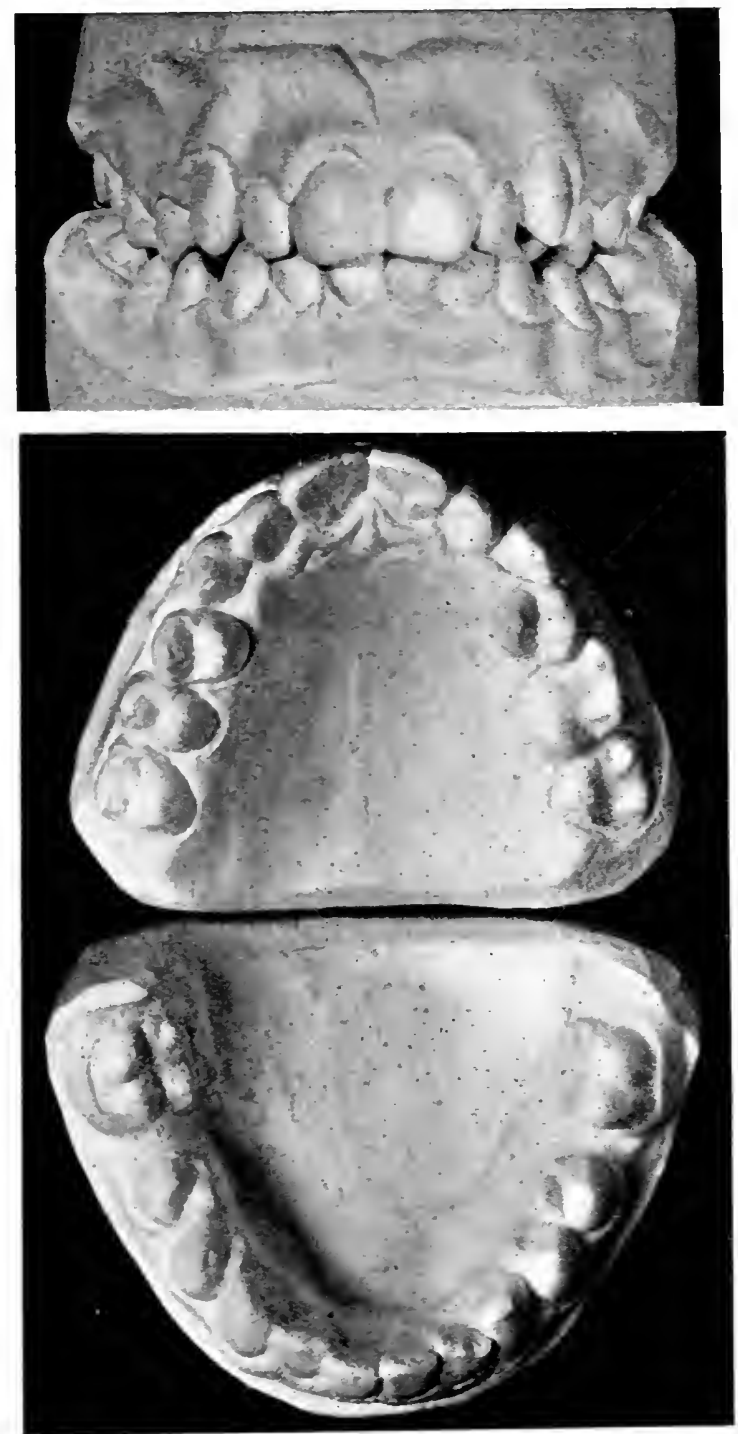

Fig. 8I.-Shows the Labial Surfaces of the Teeth when the Case was COMPleted, Which TOOK EleVen MoNths.

Fig. 82.-Shows the Palatal View of the Upper Teeth when COMPLETED.

In the lower jaw the two decayed molars were extracted only, which allowed the crowded second bicuspids to come through eleven months after, as shown here. 


\section{CHAPTER XVIII}

\section{THE PREPARATION OF CAVIT IES IN TEETH, AND FILLING WITH ZINC AND SILICATE CEMENTS}

THE conservation of Nature's own organs, the teeth, when caries has attacked and partially destroyed the crowns, is an art which is creditable to the dentist's skill, because it is constructive and reparative instead of destructive. The power of a natural tooth in mastication is really valuable to the owner, whereas an artificial tooth mounted on a plate, however artistically it may be constructed, is only a substitute compared with the efficiency of the natural one.

Thorough scaling and polishing of the teeth is an important preliminary to the proper examination of the teeth for the discovery of caries. The parts of the mouth where tartar or salivary calculus is usually deposited is behind the lower incisors, which are near the mouths of Wharton's ducts, and on the buccal surfaces of the upper molars in proximity to the mouths of Stensen's ducts. In some mouths where cavities become painful, the tartar is apt to cover over these teeth because the patient avoids masticating food on that side. Food in mastication cleans the teeth on the exposed surfaces, which are called " self-cleansing surfaces," but when food is not presented to them, they become furred over. Suitable scalers are employed to flake the calculus away either by lifting it from beneath the ledge which is on the gums or by pressing it from above towards the gums. A swabbing of pure peroxide of hydrogen (Io or 20 volumes) is applied round the necks and between the teeth and left for a few minutes. Then the use of small polishing brushes in the dental engine will cleanse and polish them, finishing with waxed dental floss silk thread or ribbon between the teeth. The discovery of cavities, by using fine probes, on the labial, buccal, and lingual surfaces is an easy matter, but between the teeth, where the surfaces vary from a quarter to half an inch, it is often a difficult matter. Transillumination with an electric mouth lamp will reveal their 
existence in some cases, or the presence of an opal-coloured patch will indicate their proximity beneath, otherwise the only means of finding them is by using various shaped probes and patiently applying them.

Unfortunately, there cannot be anything like a complete classification of cavities, because their shapes are endless in variety. But the nearest and best way of classifying them for practical purposes is, by common consent, to name them according to their position on the surfaces of the crowns of the teeth.

The surfaces of the six upper and the six lower front teeth are named-

Labial or lip surface.

Lingual or tongue surface.

Mesial or front side surface

Distal or behind side surface,

Cervical or neck surface.

Incisal or cutting edge surface.

The bicuspid and molar surfaces are named-

Buccal or cheek surface.

Lingual or tongue surface.

Occlusal or morsal or masticating surface.

Cervical or neck surface,

Distal or behind side surface.

Mesial or front side surface.

When cavities occur on these surfaces they are termed simple cavities, but when they extend to two or more surfaces they are termed compound, and are indicated by such combinations as-

Incisors and Canines.

Mesio-labial,

Disto-labial,

Mesio-lingual.

Disto-lingual.

Mesio-incisal.

Disto-incisal.

Mesio-disto-incisal.

Cervico-mesio-incisal.
Bicuspids and Molars.

Mesio-buccal.

Disto-buccal.

Mesio-lingual.

Disto-lingual.

Mesio-occlusal.

Disto-occlusal.

Mesio-disto-occlusal.

Disto-cervico-morsal

The following terms are often used in conjunction with the above to describe their form and extent:

Approximal.

Continuous.

Broken-down.

Contour.

Submarine 
The cause of caries has been the subject of many theories which have ultimately come into conflict with progressive knowledge. The theory which is now generally accepted as being correct is called the chemico-parasitic theory.

Dr. H. C. Register, M.D., D.D.S., Philadelphia, in the Dental Cosmos, December, I9I4, p. I305, gives a clear description of the cause of caries. He states: "Black, Williams, and Miller, have placed their final stamp upon the origin of dental caries as being a chemico-parasitic condition, which is now generally accepted-i.e., the fermentation of carbo-hydrate material in the presence of acid-forming bacteria, resulting in the formation of lactic acid, first destroying the enamel cuticle through the formation of plaques upon the teeth, then attacking the interprismatic substance of the enamel prisms, allowing the prisms to fall apart, and the bacteria then filling the dentinal tubuli, destroying the contents and basis substance of the dentin by acidulation. This was supposed at one time to be due to a single species of germ influence, the Leptothrix buccalis. The new school now generally accepts the views given by Black, Williams, and Miller of later date, whose observations showed a large variety of microorganisms to be acid-producers. The latest word regarding the predisposing causation of caries is said to be intimately related with the chemical composition of saliva."

The parts of a tooth liable to caries, as shown by examination of several thousand teeth out of the mouth which were split in halves, are the fissures on the occlusal surface of bicuspids and molars, revealing zones which are marked under the enamel along the dentino-enamel junction; and also the cervical edge of the enamel. These zones spread and develop, revealing themselves in the cavities which are presented to us in daily practice. In a number of instances there was practically no visible or macroscopical sign of decay, but inside there were distinct zones of caries with a dark edge immediately against the enamel layer. The thorough examination of sections of teeth under the microscope with a $\frac{1}{6}$ objective lens will reveal these vulnerable zones.

The important stages in the preparation of cavities for filling are-

I. Breaking down weak enamel walls.

2. Defining the extent of the cavity.

3. Removing or treating the dentine.

4. Preparing the enamel margins.

5. Securing suitable retention. 
A tooth that appears to be past all hope for filling presents a much more hopeful aspect when the rugged enamel walls have been removed. This operation is quickly carried out, and depends on the familiarity we have with the nature of the enamel and the instruments for doing it. If we experiment with some teeth out of the mouth, the operation is definitely demonstrated. Put some teeth into water and soak them for a few hours. Mount some of them in modelling composition or plaster of Paris as a block. Take a few of the remaining ones which have complete crowns, and carefully hold them over a tiny Bunsen flame. We shall find that the enamel will fly apart like glass. If they are gently waved in the flame, a complete enamel cap will sometimes come away. This experiment will show us the relative thickness of the enamel to the dentine underneath, and also the lines of fracture which indicate the various directions in which the enamel rods or prisms lie. If we now turn our attention to the wet teeth mounted on the block, we shall find that the enamel, which is impossible to cut with burs, will break down with ease if enamel cutters are used. There is no need for a large number of these, but a few well-chosen ones will answer the purpose. In molars and bicuspids, particularly where the cavities are deep and the pulp exposed, or nearly so, it is unwise to risk the instrument suddenly slipping and causing the patient pain. To avoid this and facilitate the cutting, we suggest the use of Ivory's square scalpers, which are used in the workroom. These scalpers are bayonet-shaped and small in size. They are held in the palm of the hand and do not alarm the patient, as the handle is hidden in the operator's hand, and if applied to the external surface of the enamel without any force and very little pressure, the rolls will fly off. The enamel must be cut without any scraping movement, as it will probably cause pain; our skill lies in lifting the prisms away by a somewhat sudden, though slight and delicate, pressure in the right direction. There are three shapes in these scalpers-one is square and the other two are diamond-shaped for right or left side. If one should slip they cannot penetrate into the pulp, as the tool is well under the control of the hand. Dr. Black's enamel cutters are excellent for finer work. The directions in which the enamel rods lie are indicated by the diagram in Fig. 88. The enamel of each cusp of a multicuspid crown is complete in itself. The imaginary or arbitrary spot indicates the point from which the angles of the 
rods will be found to lie. When the angles are thoroughly understood, a whole cusp can readily be removed with a few strokes of the cutter providing there is a cavity underneath. The smallest amount of healthy dentine will well support the enamel wall. A whole cusp under the same circumstances may be removed with one stroke of a chisel, tapped with a mouth mallet.

The limits to which the walls of the cavity shall be extended depend upon the quality of the tooth substance. If the tooth has a strong, well-developed yellowish crown, we can cut away until all traces of decay disappear, but with pearly looking teeth it is better to extend the cavity well towards the smooth and bold surface of the enamel, where the edges of the filling are more easily self-cleaned by the action of mastication. Dr. Black's axiom is indeed well founded- " Extension for prevention." It does not matter how well a cavity has been prepared and the filling condensed if this law is neglected.

If a cavity extends very near the cervical edge of the enamel it is wise to cut away the remainder, as it is also a vulnerable zone. In extending a cavity we are faced with a different proposition to that of an open cavity, but it is readily done by using cross-cut fissure burs and inverted cone burs. Cross-cut burs are very suitable for cutting enamel, as they do not really cut, but, owing to the brittle nature of the enamel, break it up. By inclining the bur at such an angle that the dentine is cut first, the enamel soon breaks down. In extending fissures on an occlusal surface, some operators regard it as an operation involving a lot of laborious work; but it is readily done by taking a flameshaped bur, such as a Humby's root roughening bur, and rubbing the point on an Arkansas slip so that a tiny chisel edge is made to it, dip this point into an antiseptic and anodyne ointment. then apply it to the fissure, when it will be found to penetrate without difficulty, and the bur is moved along until the whole is penetrated. Then take an inverted cone bur and enlarge the fissure from underneath outwards.

The question, which is often discussed, arises here: It is all very well for the poor patient to sit while we carry out such extensive cutting, but how can we accomplish the cutting of tooth tissue without pain? Much can be done by following a few rules. Firstly, an anodyne lubricant is useful, for two or three reasons. We prepare the lubricant by taking $\frac{1}{2}$ ounce of vaseline and spatulating about $\frac{1}{2}$ drachm of Dentalone into it, or, better still, about 
5 grains of paramonochlorphenol warmed into the vaseline over a hot water bath. Burs for cutting enamel are dipped into the mixture, which prevents the blades becoming clogged as warmth is generated by the revolving bur on the tissue. It is essential that the operator does his work, while using engine and hand instruments, with the aid of a mouth mirror, as familiarly as if he were looking directly at it.

In cutting dentine there are two zones where it is often sensitive. Immediately under the enamel layer at the dentino-enamel junction the dentinal tubes with their filaments branch into numerous endings (see Fig. I8). If caries is present, the filaments are in an irritated and sensitive condition. Also, where carious dentine is very near the pulp and at the cervical surface of the crown, we find the dentine very sensitive. At some time or other we cut our skin accidentally and wonder when it occurred, as we did not feel it; but if it is touched some time after, it gives us pain, because slight inflammation supervenes. When we wish to cut into a perfectly sound and healthy tooth in order to devitalize the pulp for the purpose of making a crown abutment post, we often can do it quite painlessly, and even cause the dentine to be lightly stained with blood from the pulp before the patient really feels any pain, if we use the bur lubricant, which shows that when inflammation is absent, pain is less in proportion.

In defining the extent of the cavity, if there are two or three adjacent cavities they should be extended and boldly made into one. We must not be afraid of cutting freely, at the same time we must not bore indiscriminately into the dentine; burs must be definitely controlled and made to cut the part we wish to remove. They have a way of running about with a beginner, consequently an unnecessary amount often gets cut away without any advantage, and often without any definite retention. Burs for enamel should be cross-cut, fissure shape and inverted cone, cross-cut. Those used for dentine are of various shapes, but the blades should be plain and sharply set. The cutting should be carried out with very little pressure, from within outwards. These delicate little instruments require intelligence in their use; if they are overwhelmed with stress their blades quickly get blunted, whereas if their cutting capacity is allowed free application, they will cut and grip the surface.

A good bur has a bite with it like a good razor, and shaves the tissue quickly. A bur is only capable of a certain amount 
of work, and a large cavity will use up the capacity of three new burs. Pressure applied to make a blunt bur cut develops heat, which causes pain very quickly. The various shapes of burs for dentine are of importance, but some more than others. The most useful is the oval one. In carrying out the experiment for removing the enamel layer from the dentine over a Bunsen flame, if we cut a section through the remaining dentine, we shall find that there is not much room for using round burs indiscriminately; moreover, they do not cut so well because they have a dead centre which quickly becomes blunt. The oval bur gives us a deep side cutting blade which really cuts without involving unnecessary removal of the dentine, which either weakens the enamel walls or encroaches near the horns of the pulp. The oval bur is very useful for cutting under the weak zone beneath the occlusal surface, and also for making retention pits and grooves; it combines the use of the round and wheel bur in one, very often. The use of the bur lubricant is as suitable for dentine as for enamel cutting.

Dentine is an entirely different substance from enamel, and requires different treatment. Although it is not so hard as enamel, yet it is very tough, and the enamel edges should be entirely supported by it, otherwise the enamel will fracture away from the filling at some time or other.

For practical purposes in the preparation of cavities, apart from pathological conditions, we find that the dentine is affected in three stages or degrees of destruction:

I. Contamination, where the dentine is stained but quite hard.

2. Decalcification, or the removal of the hard lime salts by chemical action of acids. It is soft and very much like hard skin or gelatine.

3. Obliteration, or débris.

With contamination, the dentine may be extremely hard, and even harder than normal dentine in some cases. This condition may be successfully treated with a penetrating antiseptic and rendered inert by applying a dressing of cinnamon oil and sealing with gutta-percha. Dentine should be cut freely in each lateral direction as the shape of the cavity will allow, but in cutting deeply we have to consider the proximity of the pulp and avoid exposing it if possible. Some pupils will ask how they can tell when they are near the pulp, as they have found in some cases that they are upon it before they have cut very far. 
Reference to Fig. 93 will show the various shapes of the pulp chamber. Generally speaking, the configuration of the surface of the cusps of a molar or bicuspid is according to the shape of the pulp contained within it. So that in a tooth with deep wellshaped cusps, the nearest way to the pulp from the central fissure is at an angle of 45 degrees. The depth of a cavity needs prudence in the removal of affected dentine, first of all to spare the patient pain and undue inconvenience, and secondly to save a busy operator's time. Decalcified dentine is a suitable material for capping the pulp, and providing it is in good condition, and there is no evidence of continued pain from the pulp, it may be left in position untouched and utilized for pulp-capping purposes, because if it is removed there is probability of the pulp becoming exposed. Artificially capping the pulp is an entirely speculative procedure, and any interference with the integrity of its position will cause it gradually to degenerate and die, with probable subsequent septic trouble afterwards. If a cavity either has a layer of white soft dentine or the bottom surface has a peculiar springy touch to it under an excavator, it must be dealt with in some way or other before proceeding further with our work. If we decide to retain the soft tissue it is tanned or mummified. The most suitable way is to dress it with tannin and cinnamon oil, which coagulates the gelatinous layer and renders it like hard leather. Good leather is incapable of becoming putrescent; it is left about in all climates and under all conditions of warmth and moisture without putrefying. Moreover, even if old leather is utilized by being ground up for manure, it is of very little value because of its insoluble nature. We hope that we have made the subject of pulp-capping clear, and the advantage of retaining a layer of dentine between the cavity floor and the pulp. Moreover, in the case of a back permanent tooth in a child's mouth where the apices of the roots have not completely closed, this method is of value as it is undesirable to devitalize the pulp under these conditions.

Where dentine is soft and pulpy it must be definitely removed, even if it leads to exposure of the pulp. At this point we must refer our readers to the chapter on Root Treatment.

The cavity having been defined, we must take measures to render it sterile. Drying it well with hot air is one means, but it is only superficial. Although the cavity may appear to have clean and healthy tissue for its walls, it cannot be trusted to be free 
from bacteria (see Figs. 83 and 84 ). The cavity must be dressed with a suitable antiseptic. This must be potent but entirely

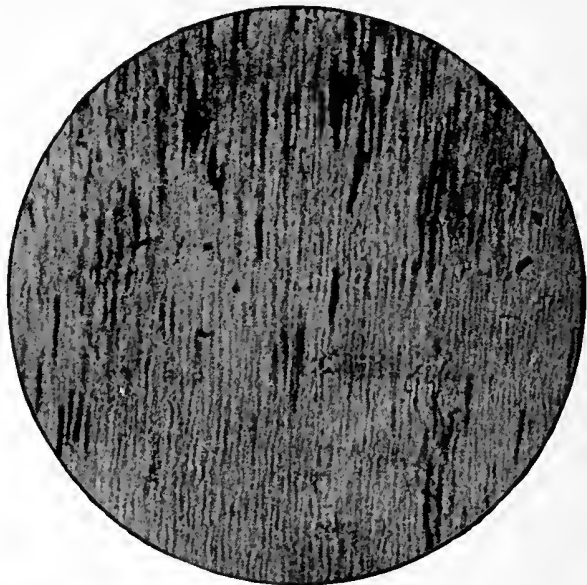

Fig. 83-Denep Portion, affected with Caries. Tubes at Intervals filled ivith Micro-Organisms. This Section to the Naked Eye appears inaffected. X I60. (Sewill's "Dental Surgery.")

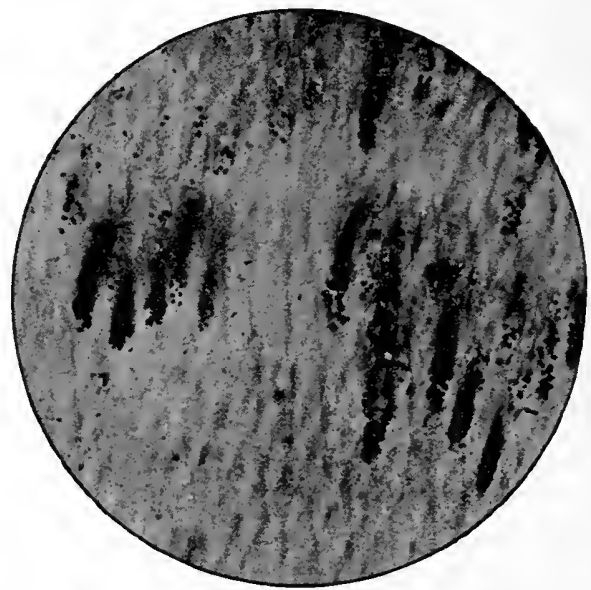

Fig. 84.-Section of Carious Dentine at Point where Diseased merges into Healthy Tissue, showing Micro-Organismsmostly Cocci-Proliferating along Course of Tubes. To the Naked Eye apparently Healthy. $\times 65^{\circ}$. (Sewill's "Dental Surgery.")

free from any irritant action, or any coagulating effect, upon pulp tissue whether upon the dentinal fibres or an exposure. 
Pure carbolic acid or pure creosote must not be used as they coagulate albumen, and directly such an agent comes into contact with the soft fibrils of the dentinal tubes it forms a number of corks or plugs, which enclose the micro-organisms in the tubuli beneath without destroying them (see Fig. 85). Also, if there is pain in a tooth from pulpitis, a coagulum is formed which prevents arsenic or any other drug from acting upon the pulp, and much inconvenience may be caused to both operator and patient. When it is desired to apply the pulp-capping method, we must first see that the dentinal tubes are sterilized, for the reason we have just stated (see Figs. 86 and 87 ). Formalin and thymol

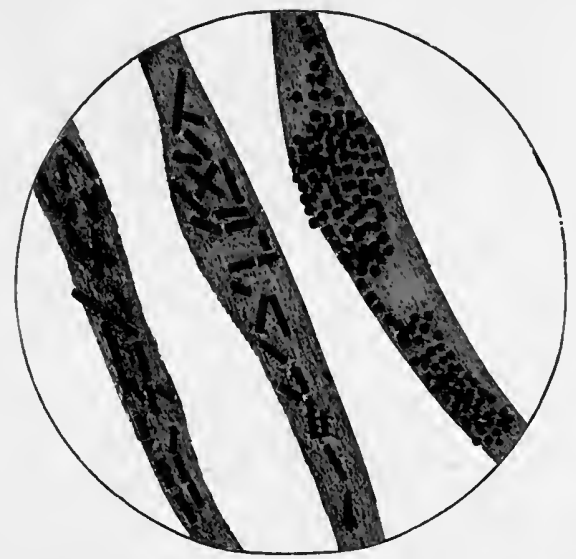

Fig. 85.-Dentinal Tubuli from Carious Dentine, showing Enlargement of the Tubes, and the Presence of Micro-Organisms within THEM. (Sewill's "Dental Surgery.")

must not be used, as they are too irritant and will cause much pain. The suitable drugs for cavity dressing are-

r. Oil of cinnamon.

2. Oil of cassia.

3. Oil of cassia and carbolic crystals, equal parts by weight.

4. Thymophen-equal parts of thymol and carbolic crystals.

5. Buckley's phenol compound.

6. Methylsalicylate two parts, carbolic crystals one part.

7. Eugenol.

8. Dentalone.

Nos. I, 2, 3, 7, and 8 are suitable for back teeth. Nos. 4, 5, and 6 are preferable for the front ones.

Whatever the shape of a cavity, maybe after it has been 
defined it must be made to retain or lock in the plug which forms the filling. Retention must be secured by making round pits

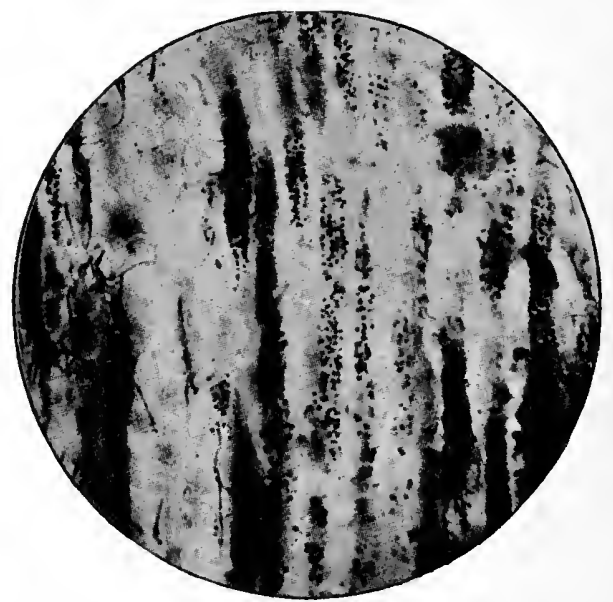

Fig. 86.-Section of Dentine in Advanced Stage of Caries. Tubes ENLARged AND FILled With Organisms, Mostly Micrococcl. $\times 650 . \quad$ (Sewill's "Dental Surgery.")

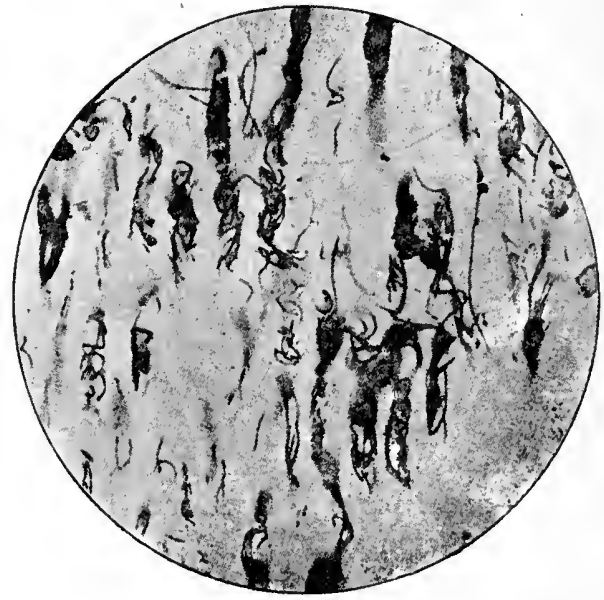

Fig. 87.-Section of Dentine in Advanced Stage of Caries. Tubes MUCH ENLARGED AND FILLED MAINLY WITH LEPTOTHRIX. $\times 600$. (Sewill's "Dental Surgery.")

and grooves at the base and sides of the cavity, also by making ledge surfaces to act as a seating which counteracts the strain of 
mastication. Care must be taken not to cut the dentine indiscriminately, but to remember the shape of the pulp chamber and make a step if necessary so as to box it in.

The enamel margins must be left nearly square and smoothed, but not overhanging.

The plugging of the cavity is carried out by using various dental materials which are specially prepared for the purpose, either chemically or metallurgically. They include:

Oxyphosphate of zinc cement.

Oxyphosphate of copper cement.

Silicate cement or synthetic porcelain.

Amalgam.

Gold.

The last three are capable of producing irritation to the dental pulp and causing pain in deep cavities, therefore it is desirable to place a lining to obviate this. It may consist of a neatly cut piece of card just the size of the doubtful spot, with cinnamontannin paste, or zinc oxide and Eugenol, or oxyphosphate of zinc cement with a little Eugenol or oil of cloves, added to the liquid before mixing. The application of a lining must be done neatly, or it will spoil the efficiency of the top filling. It is surprising how clumsily linings are put into cavities by some operators. It is quite common to observe amalgam fillings with a space round them showing cement partly washed out. When - oxyphosphate of zinc cement is used for this purpose, take a very small piece and knead it into a round ball, then carry it on the end of a probe to the cavity and neatly flatten it into place with a flat faced plugger which is dabbed into a little of the dry powder, the edges of the lining are prevented from travelling up the sides of the cavity so that the lining is shaped like a box lid. The cavity now being ready for the final filling, we must plug it with the selected material, remembering that proper condensation against the cavity walls is the next important process after sterilization to secure immunity from recurrent caries. Thorough antiseptic dressing, condensation of plugging material, and trimming of margins, are three vital points to be secured with every filling. They make a difference of years in the existence of the filling when compared with rough-and-ready methods. Fillings that only last two years are an utter waste of time and money; but when well done and the mouth is fairly normal the majority of amalgam or gold fillings should last from five to twenty years. 
There is a great difference between rough glazing and good diamond setting.

To secure proper condensation in compound cavities, matrices must be employed; the importance of this can easily be demonstrated by the number of leaky fillings one sees in daily examination of mouths. This is not always possible, but there are very few exceptions. The results of using a matrix, in the finished filling, will well repay for the trouble of fitting it, and in a great many instances saves time in plugging, especially with silicate cements and amalgams.

We will now proceed to describe the manipulation and insertion of zinc and silicate cements, for filling cavities. These two materials are much abused, and often bring adverse criticism upon conservative operations as to their utility. If the users could realize the chemical knowledge and scrupulous care necessary to manufacture a high class quality of these cements, they would be surprised at their own lack of appreciation in manipulating them. The author has spared neither time nor expense in investigating the manufacture of these filling materials, oxyphosphate of zinc and translucent silicate cements, also amalgams and root-filling materials. After experimenting with hundreds of self-set formulæ and thousands of mixes he has been successful in arriving at the finished products. To sit upon a stool while the furnace is fusing the cement day after day for weeks on end, and running into years, wondering whether the final formula has been arrived at, is a different style of labour to mixing up the product in an airy sort of way, relying upon the guarantee of the manufacturers more than upon one's own chemical skill to gain a successful result. There is very little information to be gathered from dental books or from works on chemistry with reference to the composition and manufacture of these cements. With oxyphosphate of zinc cement the mix should be hydraulic when it has set-i.e., it should set better for the final contact with water. It must not contain any trace of arsenic or alkali. Alkalis will hasten setting, but wreck the degree of insolubility required. The cement when set should swell somewhat, but this can be modified by correctly mixing it. The time allowed for manipulating it, for filling, or for fixing inlays, crowns, and bridges, can be modified by the manufacturer. This point is quite different from the hastening of the setting caused by improper mixing. When quite set, the cement should 
be neutral in its chemical reaction, and there should be no excess of phosphoric acid from the liquid as it spoils its insolubility and readily attacks the calcium salts of the cavity, thus destroying its margins. When one is familiar with the proportions of molecular structure in relation to chemical action, and the activities of micro-organisms as revealed under the microscope, we must admit that there are important points to consider in the chemistry of dental cements.

The mixing of oxyphosphate of zinc cement for fillings is apparently a simple matter. We take a smooth glass slab (we can have a dozen of these cut from a piece of thick plate glass, which saves time in a busy practice) and place a portion of cement powder upon it, then with a clean glass dropper or pipette place a small quantity of liquid by it. Draw a tiny portion of the powder into the liquid and spatulate it with a rotary movement until the whole of the liquid is engaged; there must not be a little running up the glass or agate spatula, neither must there be a leaking joint in a handle where water has collected, and trickles down the blade to the mix. When we are sure that all the liquid has been evenly engaged, we allow a few seconds for the ingredients to modify their chemical energy, then tiny portions are gradually added until the desired consistency is attained. The thicker it is mixed, the stronger it will be, but it must not be a dry mix, and its plasticity should be well developed. A small pellet is carried to the cavity with a small tantalum plugger. The under-cuts and the cavity walls are thoroughly plugged first, and the succeeding pieces are added quickly until there is an excess of material. The mass is then quickly worked towards the edges by a wiping action, so that the contoured surface is quite smooth and condensed. The finishing touches are done by smearing a plugger with a little vaseline.

Heat is a factor in the setting of cements; it can be generated by the chemical energy of the liquid in attacking the powder, so much so that careless and hurried mixing causes the mass to set too quickly to allow the proper molecular formation of the crystals which form the mass. Hot weather with the sun pouring on to the mixing slab has a similar result. The warmth of the mouth accelerates setting and allowance must be made for this. If necessary any excess may be removed with discs, or S.S. White gem stones kept for the purpose. Oxyphosphate of zinc cement secures a firm hold under certain circumstances, 
but it will not adhere under ordinary conditions. The cement, as we have already stated, swells somewhat on setting, so that it is capable of moving in ratio from the surfaces to which it has been placed, but if it is locked in the slight swelling fixes it firmly to place, so that its adhesion is secured. It is non-irritant when placed in a deep cavity, but is irritant if placed right against an exposed pulp. It is also a bad conductor of heat and electricity, which is a desirable quality.

Another factor to be considered, however, is the presence of the animal magnetism of the body. This can be discovered by a delicate sensation passing from the operator's fingers along the metal pluggers when burnishing amalgam which has reached the setting stage, and the patient winces with the sensation, which passes off when the instrument is taken away. A similar sensation is felt when the tips of the operator's fingers pass along the side of the lips against the cheek. It does not occur with every patient, but we have observed it in several cases. There is no doubt that the existence of electricity in the tissues when the saliva is slightly acid or if lactic or other acids are present in a concentrated form, in crevices, or under the sulcus formed by the gingival margin of the gum under the edges of crowns and badly trimmed fillings and inlays, will atomically remove the molecules of cement and even amalgam, which must not be confounded with the alleged solubility of the cement.

Silicate cement, or translucent synthetic porcelain, is an interesting compound. Its manufacture is fascinating, and elaborate, in its details. Its manipulation is quite worth the pains necessary to secure the best results.

The mixing slab should be of agate with a fairly large surface. The mixing spatula should be of the same material and preferably in one long piece and not with a handle. They must be scrupulously clean, and any stains from a former mix should be removed with a drop or two of hydrochloric acid and rinsed in clean water and well dried. The slab must be cool, the time of setting varies with different makes, and the directions of the manufacturers must be faithfully adhered to, because of the exacting adjustments of the chemical formula. The important points for the correct development of the silicate crystals to ensure the higliest degree of translucency and insolubility are that the cavity is quite clean and free from acid, alkali, or any foreign matter, and perfectly dry. The mixing is carried out in a similar 
way to the mixing of oxyphosphate of zinc cement. Small portions are carried to the cavity with tantalum instruments, and we must make sure that the whole surface of the cavity is thoroughly smeared with the cement and not rely on top pressure to secure contact, as this material does not swell, but, on the contrary, bad mixing will cause shrinkage at the margins. The success of the margins is definitely dependent upon complete absence of any free phosphoric acid from the liquid before setting has quite taken place, and carefully condensing the mass and trimming the edges at the time of setting. It must be completely covered-with a suitable varnish so that the moisture contained in the mass does not escape, as it is a vital ingredient in its setting, and also to prevent any moisture being imported from the saliva of the mouth.

It is impossible to insert a silicate filling successfully in a cavity with two or more open sides, as there is no way of firmly holding the mass while condensing it and the molecular formation of the crystals is being seriously interfered with. Condensation is necessary to get good results at the edges. A matrix must be neatly fitted; this is important. We use clean German silver foil of two sizes or thicknesses for this purpose, and as the material is mostly used for front teeth, we will describe the method of adjusting it. A suitable sized strip is cut and fitted to approximate to the external surfaces of the tooth around the cavity and completely enclosing it, excepting at one side to allow for the introduction of the cement. When it has been fitted accurately it is painted with a suitable varnish-liquid celluloid or clear collodion. Liquid celluloid may be prepared by placing thin pieces of celluloid into a mixture of acetone and amylic alcohol, equal parts. When the varnish is dry the matrix is carried to its place and either secured with a clamp or wedged carefully with a piece of wood, a piece of modelling composition, or gutta-percha. The filling is introduced quickly and thoroughly until a slight excess is obtained. We then have only one surface to condense upon, and the mass is wiped along with a flat-bladed tantalum plugger. When it is partially set it becomes of a cheesy consistency and can be trimmed with a sharp bladed tantalum plugger. We have four sharp blades for this purpose, and at this stage the filling is trimmed towards the edges until they are flush with the surfaces of the tooth. The matrix is removed quickly and the remaining edges trimmed if necessary. It is 
then covered with varnish and the patient directed to hold a little hot water in the mouth, and is then dismissed. The writer has had complete success with this method for the last five years, and is not worried about bad margins now. Condensation is necessary with all filling materials, and coupled with correct trimming at the time of condensation, and not allowed to stay for a day or more before trimming, will secure the integrity of adaptation of the cavity margins. Disking of the remainder of the surface of the filling, providing that the bite is not in the way, may be left for another day or so.

Another important point is that silicate cements are somewhat irritant to the dental pulp when they are placed in its vicinity. To obviate this the cavity may be painted with a little varnish, but better still a lining of oxyphosphate of zinc should be placed in the centre of the cavity and allowed to set. All cavities must be isolated and rendered quite dry before filling. The methods of keeping the mouth dry will be described in the following chapter. 


\section{CHAPTER XIX}

\section{THE FILLING OF CAVITIES WITH AMALGAM AND GOLD}

THIs chapter, although a continuation of the preceding one, includes a few points which were not discussed therein. The subject now involves a deeper aspect, as metallic filling may be used to restore any amount of missing tooth tissue which has been sacrificed through caries.

In preparing cavities during the cutting stages, some operators prefer to keep them quite dry and blow away the débris with a rubber chip blower. While dryness makes the cavity visibly clear, yet the particles of débris which float away in the air are hardly favourable to the operator's health. This raises an important point for our consideration, as it involves the question of pain. We have found that it is better to do the preparation of the enamel walls while moist, and wash the débris away with warm water from a Dr. Hunt's water syringe from time to time. This part of a cavity is cut with much less pain than when kept quite dry. The application of a preliminary dressing in a cavity, without partially preparing the cavity first, causes unnecessary sensitiveness when the patient returns for the next sitting. By opening out the enamel walls and roughly excavating the dentine the medicament has a better chance of doing its work, and the finer preparation of the cavity can be done at the next sitting. We must be careful in sealing a dressing in with gutta-percha to see that the edges of the cavity are quite flush, as any overhanging material will cause sensitiveness within a few hours. Also when this material is used in a deep approximal cavity where the gum is somewhat loose and hyperæmic it will sometimes work down on to the septum of bone. We have observed in a few cases that small portions of thin bone have become loosened in this way. Whether the bone was in a friable condition owing to constant acid reaction through the loose gum allowing fermentation to exist we cannot say. Gutta-percha is a valuable and useful 
adjunct for sealing medicaments into a cavity, but we have to be careful in its use. We refer more particularly to the softer guttapercha compounds which are prepared for this purpose. It must not be used as a lining for a deep cavity in a live tooth, as it appears under the constant warmth of the mouth to find its way microscopically into the dentinal tubes sufficiently to cause pressure, which irritates the nerve fibrils and produces pain.

A deep cavity sometimes becomes partially or even wholly filled up with hypertrophied gum, which must be removed before it can be dealt with for filling. In a few cases it may give a little trouble by its persistency to keep on developing. The removal of the gum can be accomplished in those cases where it has only partially entered the cavity by placing a pellet of cotton-wool or a piece of amadou saturated with mastic varnish and packing it tightly into the cavity. In bad cases it is better to remove it with the electric cautery, or the cautious application of trichloracetic acid; the last two methods are bloodless. In applying trichloracetic acid we place a small crystal on a glass slab and slightly moisten it with water, then saturate a piece of amadou about a quarter of an inch in size, and after having applied napkins, etc., to keep the mouth quite dry, we touch the gum with it, which turns quite white; after allowing a few seconds we repeat the wiping of it two or three times and wait for a few minutes. Its action is caustic and at the same time dries up the tissue. It must be re-applied in a day or so, and again, until the gum has been quite removed. The use of the electric cautery will remove it at one sitting.

The preparation of cavities may be partly done, as already stated, so that the débris can be washed out with warm water, but the application of medicaments and the insertion of filling materials must be done under conditions of complete dryness. This is secured by various means, and includes the use of the undermentioned accessories or a combination of them:

I. Aseptic dental napkins.

2. Cotton-wool rolls.

3. The rubber dam.

4. Saliva ejector and tubes.

The employment of aseptic dental napkins is a very useful and convenient means of keeping the neighbourhood of the tooth under treatment in a dry condition in the upper jaw. They are supplied square-shaped, and we fold one or two of them into a pointed dart 
shape. To apply this folded napkin we lift the upper lip right up with the fingers of one hand and with the fingers of the other hand we tentatively place the dart point against the top of the gum as high up as possible, while we conduct the central portion of the napkin with the third and fourth fingers to its place between the gum and cheek. We then finally adjust the point of the dart under the lip and bring the lip down and over it. The lip and cheek act as an effective clamp to retain it. If it should slip from its position and become wet, or slightly so, it will not stay in place, and we must try a fresh one. A little practice will prove this method to be quick, effective, and convenient. If necessary the napkin can be kept well back out of the way by using an Ivory's bicuspid or molar napkin clamp, which is applied with rubber dam forceps.

Cotton-wool rolls are very useful, especially in the lower jaw. Short rolls as supplied are too short for general use. If we have six-inch lengths in sizes 2,3 , and 4 , we can cut pieces to suit the case in hand. The tongue on one side and the corner of the lip on the other side can be utilized as efficient clamps to retain them. For the outside gum we retract the corner of the lip right away with a mouth mirror held in one hand, while the other hand holds a pair of tweezers which carries a piece of wool roll about two inches in length. The roll is then laid into its place by being drawn about an inch forward along the sulcus of the cheek while the lip is still held away. The lip is then drawn over the roll which holds it firmly in place. For the tongue side we cut a piece of roll about $2 \frac{1}{2}$ or 3 inches in length, weaken it in the centre by twisting it round, and contract it to a $V$ shape. Then direct the patient to put the tongue out and keep it there. In the meantime we place the roll under each side of the tongue and firmly conduct it down with a mouth mirror, and when it is nearly in place, but making sure that it is really under the tongue on each side, the patient is allowed to draw the tongue in, which effectually clamps it down firmly and securely. A little practice will allow this to be done quickly. The saliva ejector and saliva tubes constitute an apparatus which cannot very well be dispensed with in order to keep the mouth dry, although we had to work without it years ago. It is really effective in removing the saliva, which otherwise quickly soaks the napkins and cottonwool rolls, forcing us to change them too frequently. There are several shapes of saliva tubes. We require one for use in 
syphoning the saliva from the front of the mouth. When operating on the back teeth we can use a form of tube having a tongue guard, which in many instances dispenses with a cotton-wool roll on the lingual side. Saliva tubes known as Ladmore's and Sutcliff's are very efficient, and remain in position without being held. We require a large size for adults and a smaller one for children. Clamps may be used in conjunction with wool rolls, such as the Gross wool roll clamp, the Dental Manufacturing Company's Fox clamp, and Dall's tongue and lip guard clamp.

The use of a rubber dam is the most effective means of precluding moisture from the tooth which we wish to keep quite dry, and even free from vapour. Considerable practice is necessary, however, to become expert in adjusting it quickly. Care must be taken in long operations to keep an airway at the side in cases where a patient cannot breathe through the nose. The principal difficulty is where one, two, or even three teeth may be required to be let through the rubber dam, where there is apparently no room for passing it over through the crowding of the teeth. For use on the front teeth we cut a square or a triangular piece off the rubber sheet, and punch a suitable sized hole or holes with a rubber dam punch. If two or three holes are required they should be punched a distance apart corresponding to the distance of the centre of one tooth to the centre of the next tooth, otherwise when the rubber is on the stretch, the parts that go through the interdental spaces are so stretched that they fail to keep the saliva away, which renders the whole thing useless. To put it on the tooth we take the rubber and stretch it from the back to the front with the thumbs or forefingers and the nails facing each other and bring it over into place. The finger or thumb nails are held tightly against the neck of the tooth, and the rubber is allowed to close, but it must still be held until the remainder of the rubber comes to rest, when it will be found that the edges against the tooth are turned under, and thus prevent saliva weeping through. A trace of soap will facilitate the passing through between tight teeth; with a shallow tooth that keeps slipping off, if we wipe it with alcohol first, when it will probably stay in place. The main thing is to stretch it so that the sides become fine enough to pass through the tight part without breaking. Some operators separate the teeth with a piece of tape, or rubber, or cotton-wool, which is left for a day. This 
method should be avoided as much as possible, as the tooth becomes very sensitive, and especially so when the final disking of the filling takes place. With back teeth we partly roll up the rubber sheet and stretch it over the tooth in a similar manner. If this cannot be accomplished we can use one of Ivory's rubber dam clamps which have extension pieces to stretch the hole in the rubber on to, and then apply the clamp with the rubber on it with clamp forceps. When it is in place we free the rubber from the carriers, so that it will close round the neck of the tooth. We may also use any simple form of clamp to the tooth first, and then pass the rubber very carefully right over the clamp by stretching it. This last method is suitable where molars are very shallow and it is difficult to keep the rubber dam on while we adjust the clamp. Theoretically, the rubber should be put over the tooth or teeth and stay in place without clamps or ligatures, but in practice we secure the dam in order to avoid the accident of moisture getting through if it should slip, or by the syphoning action of the rubber moving up and down a little. The adjustment of clamps requires care, and consideration for the patient, as they are sometimes applied in a clumsy manner, and the patient is distressed by the unnecessary pinching or pressure of the gum. For front teeth we tie a ligature of silk or gilling thread with a surgeon's knot, which keeps it from slipping loose. If the rubber should be of poor quality, or somewhat perished, which causes it to split, it may be warmed to restore its elasticity, or we can take a very hot fine instrument and plunge it quickly through the sheet, thus forming round the hole a fine rubber ring which is very elastic. When the rubber dam is satisfactorily adjusted, we then require to keep the free ends out of the way in order to get at our work, so we catch the corners up with clips attached to a braid band, pass it round the patient's head, and tighten it up. The lower ends are then clipped to weights, which keep them down. Or, alternatively, we can use a rubber dam frame, which lies against the face, and catch the rubber sheet on to the catch points which are provided. A saliva ejector tube is then placed under the dam into the mouth, which relieves the patient from the embarrassment of swallowing as the saliva collects in the floor of the mouth.

We now come to the consideration of filling a tooth with amalgam. This widely used material has been submitted, by many years of extensive practice, to an exacting test as to its efficiency 
as a tooth saving material, and the general consent of the dental profession is that it satisfactorily answers all requirements on that point. Its colour is against its use for front teeth as an artistic restoration, but even this consideration is entitled to amendment if it is really well done and with a good amalgam.

There is no limit to the amount of crown tissue that can be restored quite satisfactorily. The qualities desired are:

I. That it shall not discolour under ordinary circumstances.

2. That it shall set before the patient leaves the chair so that the edges may be properly condensed and trimmed.

3. That it becomes very hard when duly set, which takes from two to three days, and longer before crystallization is completed.

4. That it does not change its shape by creeping or sphericizing so that the edges gape or leak at any part.

5. And that it shall expand very slightly, as some amalgams contract slightly, which is enough to cause leaking quickly.

A satisfactory alloy which answers all these tests is composed according to this formula :

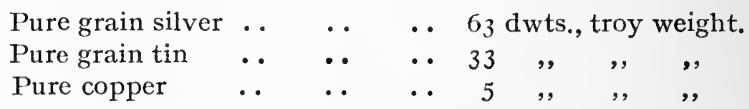

Great care is required in melting the metals so as to avoid oxidization, and as the resulting ingots are quite as hard as mild steel they must be cut by a machine. Care also must be taken to remove any oxidization on the ingot by placing it in sulphuric acid for an hour. Upon cooling, the ingot varies in specific gravity so that the resulting particles after cutting must be thoroughly mixed together and the whole of an ingot must be included. The amalgam must be kept quite dry and free from dust and exposed to air for two days before being enclosed in a bottle. It cannot be used straight away as it sets too quickly and becomes heated. At the end of a week it is quite ready for use, and does not change in any way after that time. This formula has been used for ten years under all conditions and tests and gives every satisfaction in fulfilling the qualities laid down, especially for large contour work in molars and bicuspids. For use in front teeth where it is desired to build up a corner, the same formula may be used with the addition of 5 dwts. of pure gold. The colour is enhanced, and it takes much less mercury to mix it. No special precautions need be taken as to the amount of mercury used beyond the fact that it must not contain any free mercury, 
yet, on the other hand, it must not be too dry. In building up contours, we need to vary the.consistency of an amalgam; the first portions should be plastic, but as we properly ccndense the filling, the top surface becomes too plastic, and upon completion we take this surplus and mix a little more amalgam into it, which secures good edges. It is important that we mix amalgam with pure distilled mercury, which has been electrolytically purified.

The preparation of the cavity must be faithfully carried out according to the description given in the last chapter, but as some cavities may be very deep, and root treatment has been done, we must pay strict attention to the seating of the plug. While we are cutting we should endeavour to visualize in what way pressure, in mastication, might tend to dislodge it from its moorings, because even if it did not break away, the chemical cystallization that appears to go on with amalgam might have a tendency to alter the margins. A large contour filling secured by anchorage at the base only, should be estimated for against the strain and stress of mastication in a similar way that a lighthouse is seated upon a rock, according to its levels and the top weight of pressure from storm, stress of wind, and water.

The vulnerable part of a cavity situated near the base of a tooth is the cervical edge, so that it must be cut down to healthy or clean tissue. A small continuous groove or gutter is then cut into the dentine, a little away from the external edge. Make retaining pits, not very deep, where the dentine is strong, and, if necessary, an undercut groove where practicable. If the pulp has not been involved, amalgam may be plugged into the cavity after suitable dressing has been sealed in for a day or so. The cavity should be cleaned and dried, then swabbed out with a suitable antiseptic, and roughly dried so as to leave a trace in it. If the floor of the cavity is very near the pulp, and it feels somewhat springy under an excavator, it may be capped with a piece of card carefully, and neatly cut to the size of the doubtful spot, and cinnamon paste put on to it and applied to its place. Amalgam can then be packed in small portions around the card cap and then over it until the cavity is filled. If it is at all doubtful, a lining of medicated cement should be placed on top of the cap. A suitable lining cement is made by adding a little oil of cinnamon or Eugenol, on the mixing slab to the cement liquid before mixing it with the powder. The application of a lining requires neatness, or the cavity becomes smeared in an uncontrollable way, and will 

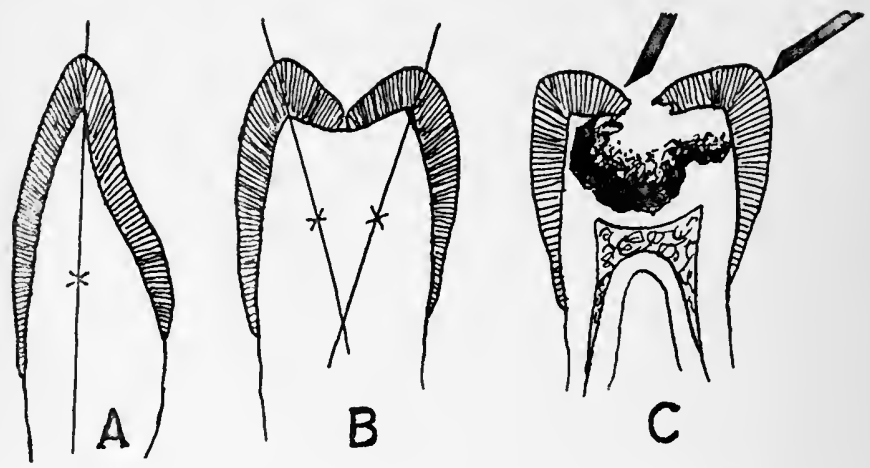

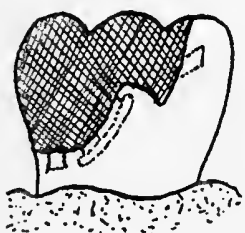

D
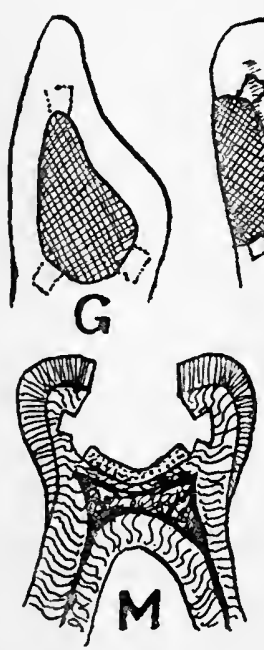

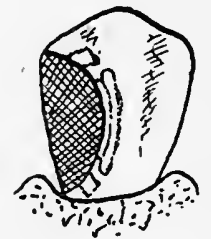

E

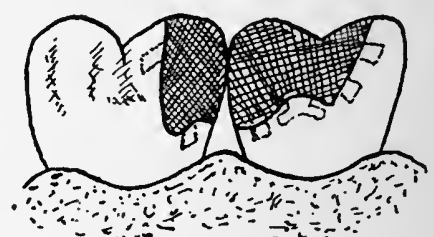

$F$
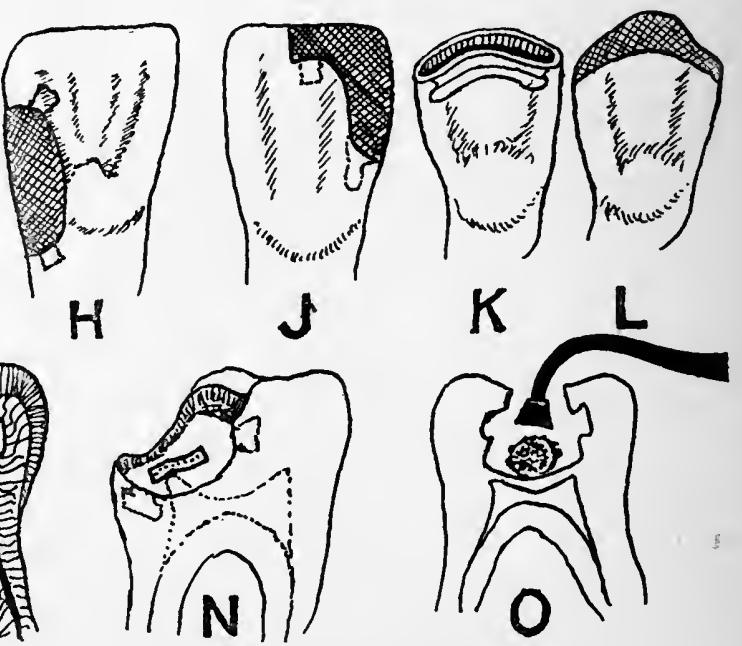

FIG. 88 .

A, B, C, angles and lines of cleavage of enamel prisms; D, E, F, amalgam fillings, showing principles of retention, pits, grooving and seating; $\mathrm{G}$ to $\mathrm{L}$, gold fillings, retention and margins; $\mathrm{M}, \mathrm{N}, \mathrm{O}$, capping and protection of the pulp. 
cause leakage of the top filling. A little cement having been mixed, we take a tiny piece, forming it into a ball, carrying it with a point to the cavity, flattening it down with a flatfaced plugger and shaping its sides according to the formation of the cavity; a little dry cement powder on the instrument prevents its pulling away. If more cement is needed, then place another tiny ball in the cavity, shaping it like a box. If the crown has broken down very badly and root treatment has been carried out, a metal post or a loop can be inserted in the sealing cement which fills the pulp chamber and partly down the roots. Difficult compound cavities and large contour fillings are rendered straightforward like a simple cavity if a matrix band is properly fitted and secured with a simple but effective matrix clamp. Carefully made matrix bands give such perfect results while condensing the amalgam that it is imperative we become expert in adjusting them. The evidence, as shown in a number of fillings that we have seen presented to us in daily practice, is that either no matrix has been used, or the salient features of a matrix have not been secured, or that the condensation of the amalgam has been overlooked. There are several good matrix clamps on the market. Leonard's matrix clamps for bicuspids and molars are excellent, as the jaws are really shaped accurately to fill the interdental space so as to hold the matrix band in place. Harrison's nickel ferrule matrix collars are also excellent, and very simple to apply. They can be adjusted so that the interproximal space is not trespassed upon in any way. A collar is selected somewhat larger than the tooth crown, and with a pair of pliers the cervical portion of the band is caught up, tightened, and made into a lap; the upper portion is then caught up, and turned into a lap. The lap is then turned over, which secures the collar into position, and the contour of the tooth is correct as far as the cervical portion is concerned. When the filling is completed the lap is unfolded and the band opened out somewhat so as to loosen and facilitate its removal without dragging part of the filling away. The cervical portion only needs a bladed burnisher to complete its surface. The upper or proximal surface can be trimmed and then burnished. The important point in the use of any matrix is that the cervical edge must be flush with its surface. A parallel band is useless as a matrix. The band must be shaped at its edge to accommodate the cervical margin of the gum, and to 
enclose the whole of the cavity, however deep it may be, so that there is no space between the cavity cervical edge and the band. The writer is convinced that this point is vital to the life of the filling. So many fillings have been examined in teeth that have
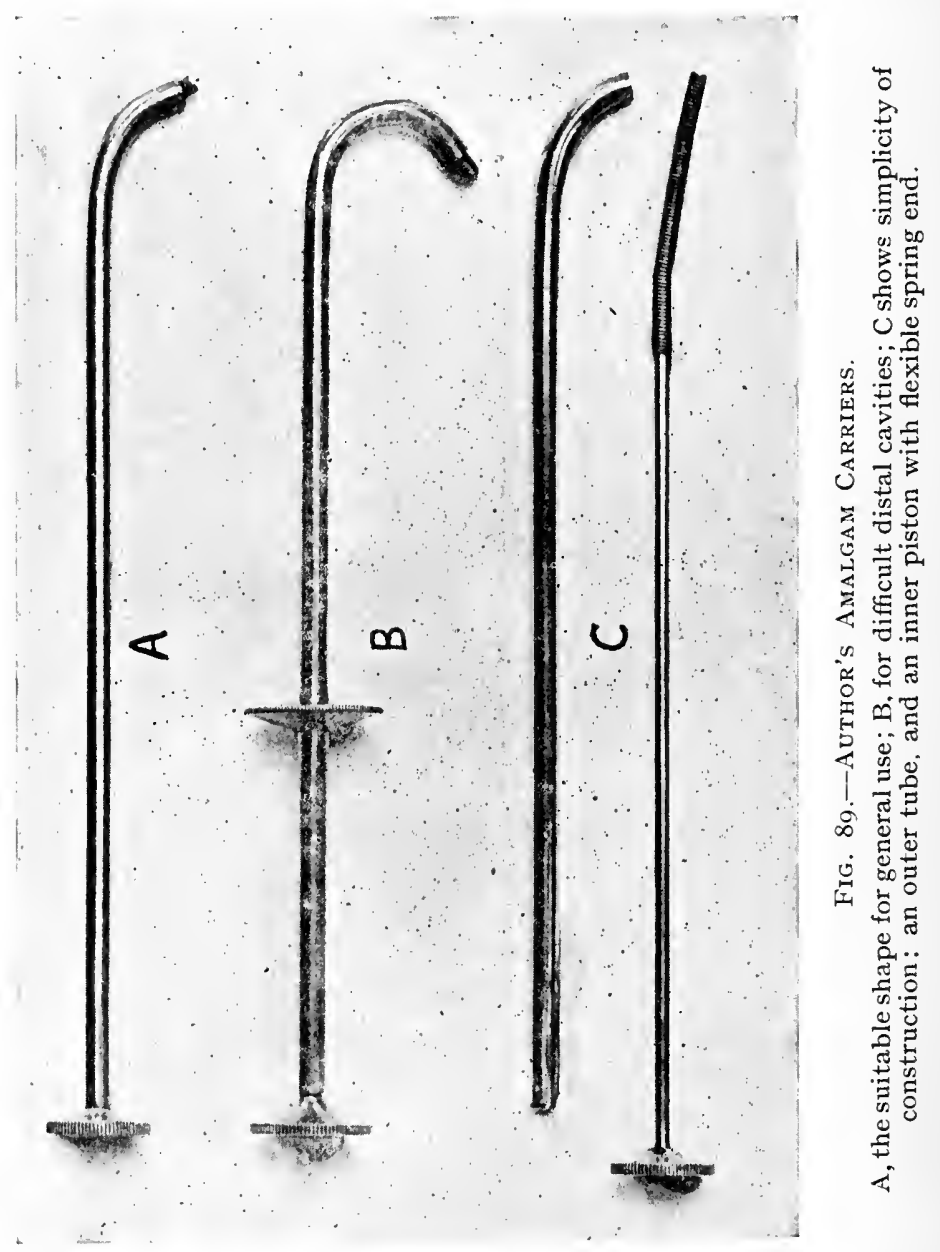

been presented to us by patients who have complained of pain, and we have found the cervical edge standing right away, in some cases nearly an eighth of an inch, with a fresh cavity leaking badly beneath it. This shows that the operator either used a straight matrix or failed to trim the edges correctly. Now it is a 
difficult matter to trim properly the cervical edge of a filling, and almost impossible to condense amalgam where we have endeavoured to show that it is a vulnerable and vital part, because condensation is one of the main principles of success.

Amalgam is a peculiar material for condensation; it is not like putty, which can be pressed into place. A mass under condensation is only acted upon superficially, and it spreads or slides laterally, which is no condensation at all if it is not counteracted by a fixed wall to condense against. If large contour fillings are inserted in teeth out of the mouth mounted on a block, it will be found that, even with ordinary care, it is a somewhat difficult matter to secure an absolutely non-leaking edge when using a matrix. The only way to secure absolute contact is by using small pieces of amalgam and with small pluggers burnishing them round the walls of the cavity with a wiping movement, and also against the cervical edge with a flattened edge plugger. Amalgam, when being carried to a cavity, should not be allowed to drop into the mouth so as to be infected from the saliva. An effective amalgam carrier should be used (see Fig. 89). The pieces of amalgam are added until the cavity is overfilled. If there is any over-soft surplus it is better to remove it and add a little more amalgam to it and then condense it. This soft surplus is not free mercury, but a mixture of it with the tin, which the mercury dissolves rapidly. The theory of the setting of amalgam is that the mercury, whose setting point is very low, attacks the other metals and fuses them, thereby lowering their fusing-points and raising its own; thus an average fusing-point is attained, and the setting is brought about by their secondary cooling.

In addition to the matrices and matrix clamps already mentioned, there are many cavities which require special matrix bands and suitable clamps to hold them. The principle of their adjustment to the contour of the tooth is the same, and must be faithfully carried out. The writer prefers to make a matrix band for every case, which is easier than using many ready made ones (see illustration in Fig. 9o). The band is cut to suit the case in hand, or a model may be taken of the tooth, and a band made to fit it in difficult cases. German silver foil may be used for contouring front teeth, and painted with a suitable varnish for silicate cements, which will not discolour the filling. For amalgam contour work gauges Nos. I and 2 are used, also ferrotype plate, such as is supplied at photographers' stores. The enamel is burnt off with 

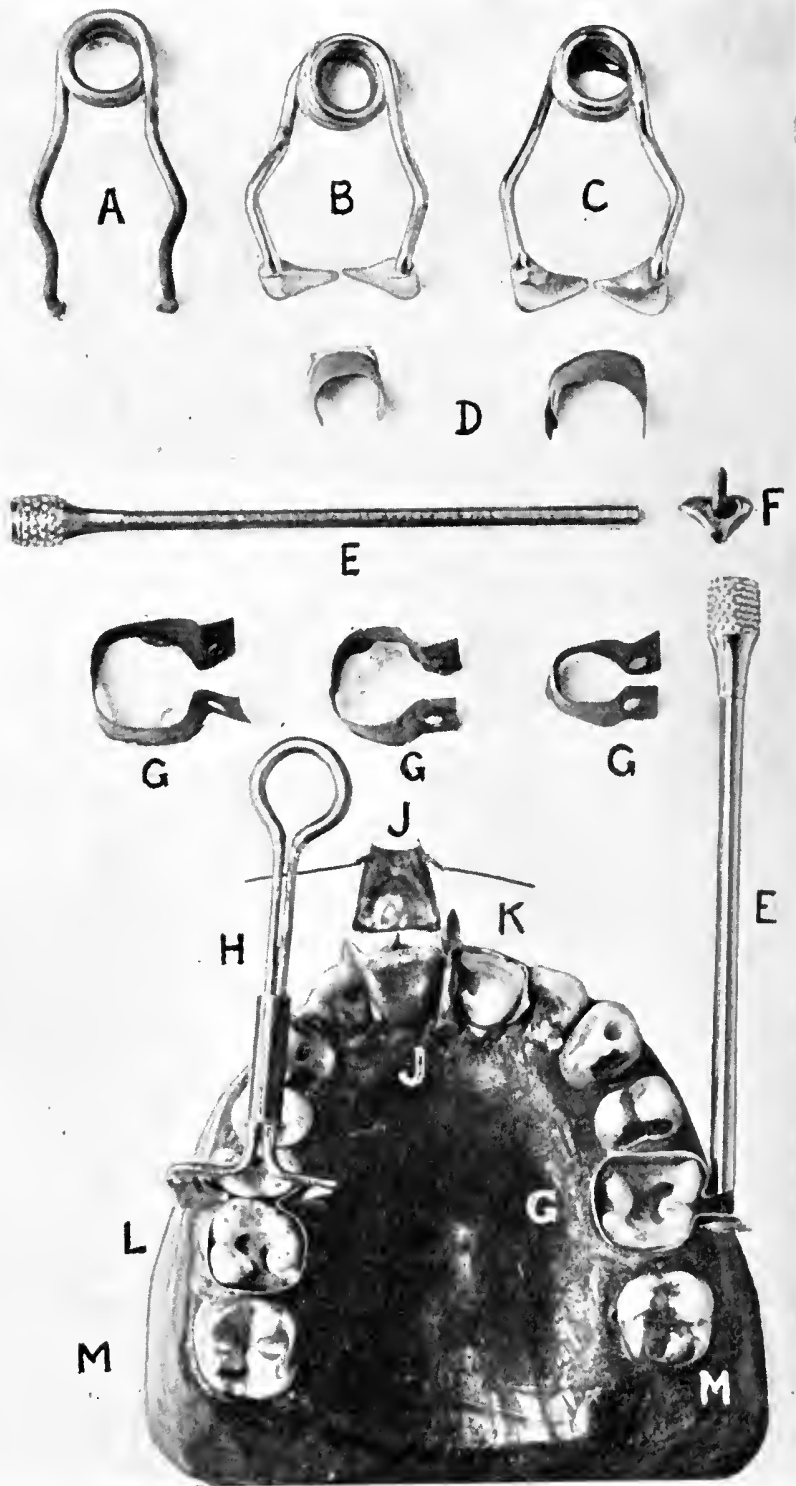

FIG, 90. (For description see opposite). 
the blowpipe first, and cut into small oblong pieces for putting into the cabinet drawer. This material is thin and yet has some body in it so as to keep the desired shape while plugging. With its use a matrix band may be adjusted to a suitable stump, and an amalgam crown built right up. When the top of a large filling has been reached, it is surprising how much condensing it will take; we use a flat blade and go round the sides of the amalgam to give it the necessary contour from the approximal surface, against the matrix, to the occlusal surface. A large round or oval burnisher is applied to the occlusal portion, and the surface is shaped as desired, and the edges trimmed. On removing the matrix band be careful not to damage the filling, and see that the patient does not smash a corner off by biting suddenly on to it if the bite is too high. It needs carefully trimming with a large spoon-shaped trimmer until the bite is quite free. The cervical edge being accurately condensed should not need any or very little trimming, a thin bladed burnisher being all that is required to flush the surface at the edges. The filling should be trimmed at the time so that it may be creditably left at that, but it is better to bring the patient back after two or three days and disc it with carbo discs, not too coarse, and finish with very fine sand-paper ones, and finally with crocus discs, which produce a brilliant burnish. A polished surface helps the filling to be bacteria proof, as the surfaces are rendered more self-cleansing. Polishing strips may be also used in the interdental spaces. For smoothing and polishing the morsal surface we use a large round bur, and fine round carbo stones finishing with a round (not ribbed) steel burnisher, or an Arkansas stone disc. Unfortunately we have not seen a cup-shaped disc on the market; the ordinary flat ones are useless on curved contoured surfaces, as they will ruthlessly destroy the contour, and then miss the cervical surface at the edge by more than an eighth of an inch.

Fig. 90.-Author's Simple System of Matrix Clamps.

A, German silver wire bent into shape with two full coils of spring with ends bent. Any size or shape of clamp jaws can be soldered on to them while placing the spring end in wet sand; B, jaws for a small space shaped to take the interdental space; $C$, the same as $B$, for larger spaces; $D$, pieces of matrix metal for bicuspid and molar; E, Dr. Huey's mandrel with ivy-leaf-shaped screw head, F, and a handle; G, matrices for large contour fillings, with holes punched to take screw clamp, E; $H$, simple spring clamp with pointed jaws to clamp up matrix, $L$, where the space is too close to admit of $\mathrm{B}$ clamp; $\mathrm{J}$ and $\mathrm{K}$, thin German silver jackets varnished for silicate fillings; $M$, completed amalgam fillings. 
The nearest thing is a cup-shaped copper disc, using it with fine carbo powder or pumice. But this is only a makeshift, as it is too stiff, and does not uniformly hug the surface to produce a polish.

Gold is the oldest filling material, and is still the standard for displaying real skill in filling cavities. There is a difference between gold fillings which are inserted for the fee only in view and those properly condensed and constructed on scientific principles. It is true that it involves laborious work, especially in a busy family practice, and the insertion of a gold inlay, cemented in, is justifiable in these busy times. Now there are several limitations to the efficiency of a gold foil filling, but many gold inlays are infinitely worse. The careful casting and insertion of gold inlays, is, indeed, an art that does credit to the dental profession, but there are so many which depend upon the cement for their hold. The anchorage of an inlay must depend upon seating, which counteracts the stress of mastication, and the spreading of the gold, which often ultimately dislodges it. The only way it should be possible to come out is by the one parallel path which allows for the withdrawal of the wax pattern and the finished casting. The vulnerable cervical edge needs super-care.

When we review the history of the art of gold filling we find that there were many early operators who only had non-cohesive gold to fill with. They had no rubber dam, no saliva ejector, no cohesive gold or any crystal or precipitated gold, and yet their fillings stood for twenty years and more. This makes modern gold filling appear as child's play compared to it. We do not suggest any comparison with the amount of work that has to be done to-day and the lucrative operations of their time, but the fact remains that they daily demonstrated the efficiency of gold fillings carried out without modern facilities.

Gold filling, of course, requires constant practice to become expert in its execution, but it still stands at the top of our art because it embodies the skill that is involved in the true principles of filling cavities, and if it is not faithfully carried out, there is no material that betrays bad work so quickly. If we become master of gold filling, we automatically become successful in filling with the other materials.

There are three forms of gold used in filling teeth.

r. Non-cohesive.

2. Cohesive.

3. Crystal or precipitated. 
The gold used is of the purest quality, and all traces of impurities are eliminated. The first two forms are prepared from molten gold which is cooled, rolled, and then beaten out between vellum until the sheets are very thin.

Non-cohesive gold consists of the gold sheets or foil as they leave the goldbeaters, and is not annealed. This form or condition exhibits a curious quality inasmuch as gold plate, when it is rolled or otherwise worked, becomes springy and brittle, but when beaten out into foil it develops a very soft quality. Non-cohesive gold is capable of being folded into strips, interlaced, and wedged, while still retaining its soft quality. The sheets may be manipulated as plain foil, or rolled into cylinders. As the pieces of gold are not capable of cohering or welding one upon another, it is obvious that the only means of binding the plug into one piece to remain in its position is to arrange them in such a way that no part can break away from the cavity during mastication. A matrix may be employed with the object of securing good edges. To illustrate the principle, it consists of packing and condensing the pieces as if they were heavy sacks being loaded upon a van with the tail-board let down and the sacks must not slip down in transit. If one sack should become loosened and fall out the whole load comes to pieces. But with practice and ingenuity it becomes very interesting work. In laying the cylinders into a four-sided cavity they should be placed so that they project somewhat beyond the walls and edges so as to allow for proper condensation of the surface. When plugging is complete it is trimmed with discs and finishing burs, care being taken not to tear the edges by using too coarse discs.

Cohesive gold exhibits qualities as a filling material that are entirely different from non-cohesive gold. Cohesive gold is produced by gently annealing non-cohesive gold, which should be brought to a cherry red. If overheated, it becomes very harsh. The use of cylinders is usually more convenient than plain foil, although some operators prefer it in that form, because it saves time and we become familiar with the working capacity of the various sizes. The only objection to the cylinders is that they have a tendency to become ridgy on the surface of the filling, which is undesirable, but this can easily be controlled by practice. Also care must be taken to see that the whole of the cylinder is annealed, as there is risk of it having a cold spot through the instru- 
ment that holds it not being at the same temperature as the rest. A very fine steel point such as Weston's plugging assistant, or a very fine pair of tweezers, are suitable for the purpose. Cohesive cylinders are supplied by the makers, but they should be slightly warmed before using by placing them on a mica tray over an annealing lamp. The gold after annealing will be found to be cohesive-i.e., one piece can be made to cohere to another piece and become solid with it. It is not soft like non-cohesive gold, but is somewhat harsh and is only capable of limited working. A mass of gold may be built up without any limit as to size. Although this is apparently a very simple matter, yet there are difficulties attending the process in making a perfectly watertight plug. The cylinders must be deftly handled in conveying them to the cavity, and must not be crushed in any way, as the cohesive quality is impaired instantly. If it is desired to flatten a cylinder, it must be annealed again. The cylinder also must be fixed in its position by one firm blow of the plugger, and the remainder can be plugged without fear of its shifting. There are two forms of pluggers in general use, one is manipulated by hand, and the other by an automatic mallet. There is also an automatic mallet worked on the electric dental engine. The hand plugger is useful when working near or against the margins of the cavity. The automatic plugger is more suitable for the general surface of the filling.

Crystal or precipitated gold is not produced directly from molten gold, but undergoes chemical treatment either from a solution of pure gold electrolytically, or by treatment with mercury, which is afterwards driven off by heat. This form of gold combines in itself a modification of the qualities of cohesive and non-cohesive gold. It is advisable to warm it slightly on a mica tray before using in case it should contain traces of moisture, but we must avoid heating it directly over a flame, as it makes it harsh. It is soft, and although it is not capable of being indefinitely worked like non-cohesive foil, yet it will yield quite sufficiently for our requirements in order to secure good non-leaking margins. It is quite cohesive, and apparently easy to work; but it needs much more condensing than cohesive foil. The drawback to its use in making large contour fillings is that it lacks strength, owing to its molecules being held loosely together through its chemical formation. The enamel margins, moreover, while they may be watertight, yet in many cases show a tint 
which indicates that traces of the chemicals are not always absolutely eliminated from the gold. It is a most useful form of gold nevertheless, and is quite suitable for a base in cavities with a complete continuous wall or nearly so. In large contour fillings we have to consider the top strain of mastication, as the filling is liable to break or fracture away from it. It is also useful for combination with non-cohesive cylinders in many cavities.

We have at our disposal, therefore, three forms of gold for our purpose, and an intelligent mechanical appreciation of their working qualities will enable us to build up a strong filling. There are a few points to consider as to the causes of failure in gold filling.

I. The rubber dam must be successfully applied; there must not be any weeping through of saliva anywhere, because even if it does not reach the cavity, the warmth of the mouth will cause vapour which will destroy the cohesive surface of the gold. Also we must avoid vapour from our own breath if working at close quarters.

2. Cohesive and crystal gold must not be over-condensed, or its cohesive surface is apt to be destroyed. It must be consistently or evenly condensed, but there is a time when this must be stopped, which can only be grasped by practice.

3. The margins of the enamel must not be starred, as there is danger of pulverizing them, and the resulting powder, however slight, will destroy the working surface. The margins must be smooth and slightly bevelled outwards before commencing on the gold.

4. The cavity with its anchorage for a large contour filling should extend into the dentine in proportion to the amount of gold exposed outside, which, of course, is unsupported.

5. Strain and stress must be reckoned for and a contour filling should be ingeniously built up by varying the laying position of the cylinders, on the principle of the sacks in the van which we have already mentioned, but in this case we are loading them up one upon another. We must secure a good foundation at the cervical surface of an approximal filling, and keep the outside building line well up with an inclined ledge for the upper structure to rest upon, and to counteract the stress of mastication, which naturally tends to break sloping lines of fracture away. The building up edge is secured by letting the cylinders stand 
away slightly, and now and again shaping it with Mordaunt's blade pluggers. Matrices may be used with cohesive gold, but they must be shallow, to ensure condensation at the cervical margin only, as they obstruct the pluggers and are more in the way than is welcome in this class of filling. When the filling reaches say half-way up, a deep ridge may be shaped to jut out from the centre of the tooth towards the proximal edge, and then the cohesive cylinders may be so placed until the incisal surface is

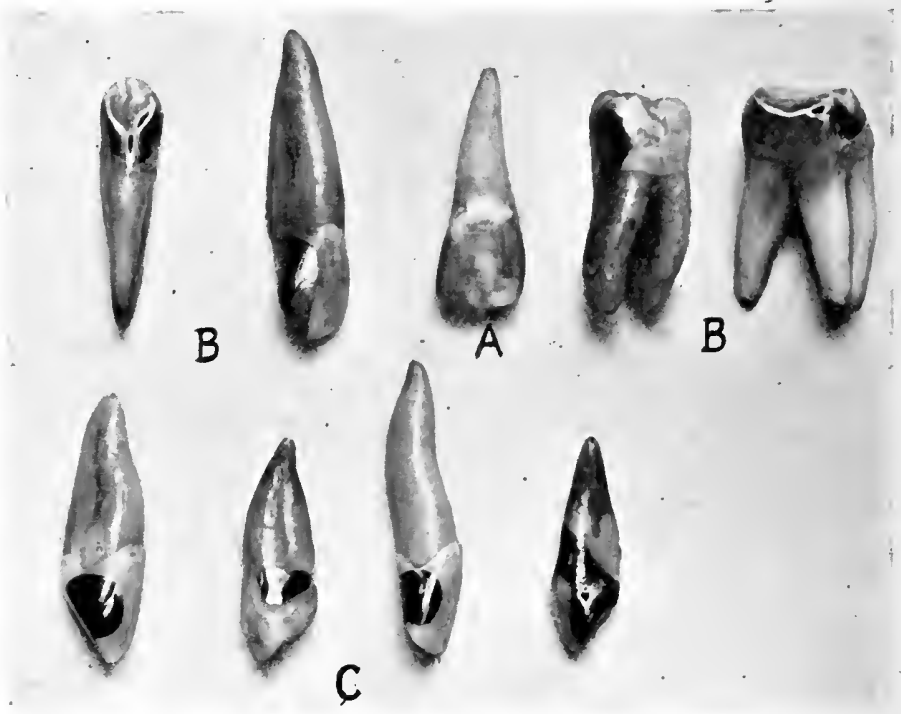

FIG. 9I.

A, Two completed silicate fillings in a central; B two canines and two molars with large contour fillings completed; $\mathrm{C}$. four gold fillings completed, the right end one involves the labio-cervical approximolingual surfaces.

reached. Butler's foot plugger point in the automatic mallet is very useful where we have a fairly flat surface to condense upon. With all cavities it is desirable to secure the margins first and to keep them higher than the central portion until the filling is completed.

There are many shapes of hand and automatic plugger points, showing that various operators prefer shapes that appeal to their personal convenience in working. We prefer to use hand pluggers with a springy rocking motion, and those in the automatic mallet with a flat motion. The instruments must be deftly 
used in order to secure the best working surface, and to make sure of non-leaking margins. The fillings are then trimmed and polished with discs and strips, which must not be coarse or the edges will be ripped away. They are then left with a mat or dull surface, or burnished with crocus discs or steel burnishers. Before a student commences working with gold it is a good plan to practise out of the mouth on cavities with S. S. White's tin foil, No. 4. This material is semi-cohesive, and fairly large cavities may be built up with it. Some old pluggers will do, as they become burnished with the metal. The advantage of practising with it is that it is only semi-cohesive, and the strips should be entangled by laying stars of strips in, and slightly folding as well as condensing them. Moreover, if the filling is over-condensed the original metal in the solid form is soft, so that it will spread out and move out of position. When the plugging of tin is mastered, the student is ready to appreciate the working qualities of gold foil and cylinders. 


\section{CHAPTER XX \\ ROOT TREATMENT AND FILLING}

WHEN a cavity has penetrated so deeply into the dentine that the safety of the life of the pulp is endangered, or if the tooth has been mechanically injured so as to produce pain, we have to consider the advisability of devitalizing the pulp and filling the root canal, or canals, so that the periodontal membrane in the vicinity of the apices of the roots shall not be infected by putrescent matter which probably would subsequently develop from any fragment of pulp tissue that might be left behind. Or a tooth may already have lost the pulp through one cause or another, and the roots may either be in an abscessed or in a harmless condition, but which, nevertheless, demands suitable treatment to avoid infection passing from the débris contained in the canals to the periodontal membrane outside.

The subject of root treatment, therefore, may be discussed under two divisions:

I. The devitalization of the pulp and root filling.

2. The treatment of dead and abscessed teeth and subsequent root filling.

I. When we have condemned the pulp we can adopt one of the following methods to devitalize it:

a. The application of arsenic or arsenious acid.

$b$. The application of pressure anæsthesia.

c. The administration of nitrous oxide gas.

The principle involved in devitalization of the pulp is the same with each method. The adoption of different means leads to the same cause of destruction. It consists of the mechanical disorganization of the vessels contained in the pulp. The anatomy of the pulp should be understood first before embarking upon root treatment. The pulp is an organ contained within the central portion of a tooth crown and its roots. It consists of a sac which is complete in itself. It contains nerve fibres, arteries and veins, fat, albumen, and a few other chemical constituents 
in small proportion. Its shape or form is an important matter for our consideration. It consists of a body, which occupies the pulp chamber at the base of the crown; it has tails, which occupy the root canal, or canals, of the root or roots; the ends of these tails have one, two, or even several hair-like extensions which pass out through the apical foramina; these ends are fibres, veins and arteries, which come from the larger branches of these vessels outside the roots. The apical foramen or foramina of each root, for practical purposes, must be regarded as being the boundary of the pulpal organ (see Fig. 93). The pulp also has hair or fibres arising from it, all round, running in various directions in an endless number, which constitute the dentinal fibrils containing nerve fibres which occupy the dentinal tubuli. These fibres split up at the ends into tiny branches, which make practically a continuous sensitive surface against the enamel layer of the crown of the tooth and also near the surface of the dentine of the root. These fibres, which occupy the dentinal tubuli, are the part of the pulp with which we come into contact in cavity preparation, and they require our vigilant attention to prevent pain from irritation that may arise from our filling or from the continued action of micro-organisms which we may have left alive through neglect of proper medication. In principle we have to carry out pulp treatment even when preparing a cavity that does not apparently involve the pulp. The body of the pulp has horns where there is more than one root present; they lie towards each cusp of the crown. In some upper bicuspids they may be exposed by boring with a bur at an angle of 45 degrees from the central fissure of the occlusal surface. The pulp terminates at the ends of the pulp canals, but its nerve fibres and bloodvessels pass out through foramina which are situated without any definite relation to the axis of the canals. This is an important point for us to grasp, as it seems to get overlooked, both with reference to instrumentation, and the ultimate root filling. To emphasize the significance of this part of the anatomy of the pulp canal, we will take the case of an apical abscess in an upper front tooth. In opening up the canal to reach the abscess we must take our nerve canal drill, which is very fine, right through, but not beyond, the apical end, if we are to drain it with paper points as should be done. We cannot always do this readily, and have carefully and patiently to drill until we feel the drill has passed through. Experience 
brings a sense of intuition to the hand which readily tells us the difference between tooth and bone tissue. We are aware that some operators are afraid of doing this, but it is the only logical means of draining and treating an apical abscess sac.

We have to run risks in root treatment because the nature of the work imposes it upon us in almost every case. It exercises our judgment, and experience is the main factor in guiding our diagnosis and treatment. Therefore, if we suspect the existence of an apical abscess sac we are running an unnecessary risk if we fail to drain it. It is not a sufficient explanation to say that we found no trace of pus in the canal after reaching the end of it with a nerve canal drill. If there is tenderness and a sense of fulness at the region of the apex of a root when a finger is applied to the external surface of the gum or face, we have reason to suspect an abscess sac being present. If we leave it, thinking that it is tenderness of the periodontal membrane and will pass off after root filling has been completed, we must not be surprised if trouble develops.

In the course of our experience we have had several cases which we were inclined to let pass, but, owing to suspicion, we adopted the method of drilling through the end of the root, and in each case the amount of pus that was instantly released was alarming, rolling down the canal before we had time to apply a paper point. Two of these cases occurred in patients who understood the nature of the operation. One was a dentist for whom we carried out an amount of conservative work with some root canal treatment in three teeth. An upper dead left lateral root was under treatment for three weeks. The canal became sweet and clean, and we prepared to fill it for trial only, but our suspicion was aroused by a slight tenderness at the apical area. We decided to drill through with a fine Beutelrock's nerve canal drill, with the result stated above. The lateral was further drained and treated at subsequent sittings, until pus had ceased and all tenderness had subsided. We finally filled and crowned the root ten years ago, and it is still in the mouth without having given any further trouble.

The second case was a medical man who, two years previously to coming, had a silicate filling placed in a cavity situated at the disto-cervical corner of an upper left central. The filling was still in position. There was no sign of leaking edges, and the plug looked perfect. Whether the operator who did the filling 
had taken precautions against irritation of silicate cement we cannot say, as we left it in its place. The patient was in great pain and the base of the nose was pushed forward from the pressure of the swollen condition of the labial tissues. The tooth being a good one, long, well shaped, and of good colour-the patient desired to have it saved if it was practicable. The swelling extended from the right central along to the left canine. The left central caused great pain when touched from the labial surface inwards, but not so when touched in a vertical line. The tooth being a good colour showed no sign that it was a dead one. This was a case which put our experience to the test. The patient was in great pain, and on the previous night had asked a medical colleague to lance the swelling, but no pus came away. We decided that the trouble was caused by infection from the dead contents of the canal of the left central, and that it must be opened. After cutting through the enamel with a tiny carborundum stone in the engine, we took an oval bur and bored at an angle of 45 degrees and struck the top of the pulp chamber, which was partially filled with débris. With a long cross-cut root bur we cut a deep groove from the pulp chamber to well into the incisal edge to secure a direct line with the axis of the canal. The canal was enlarged, with a Gates-Gliddon canal drill, about three-quarters of the way up. No pus was found, and we decided not to go further at that sitting, but applied a dressing of Black's I-2-3 and sealed it in. Next day the patient was still in great pain, so we opened up the canal with a Beutelrock's canal drill to the end, but no trace of pus was found, and we applied another dressing of Black's I-2-3. On the following day the patient had no relief, so we decided to drill through the apical end with a fine Beutelrock's right-angled nerve canal drill. We may mention here that nerve canal drills are not always long enough, and should be supplied in two lengths for straight and right-angle hand-pieces. This tooth was a full inch and a quarter in length, and we only just succeeded in getting the drill through, and it was the only one we had in stock that was sufficiently long for the purpose. The drilling had to be done patiently, with bur lubricant on the drill, and with alertness; two fingers of the right hand, which applied the drill, rested on the incisal surfaces of the lateral and canine, which prevented any sudden slip through. When the drill was just through we withdrew it, and before a paper point could be applied the pus rolled down and enveloped the 
crown of the tooth and the gum. We used about fourteen long Darby paper points, which were soaked, and the last ones were not very much less than the first ones. Johnson and Johnson's paper points are stiffer and pass up better, but they were not long enough. We poured about a teaspoonful of peroxide of hydrogen (Io volumes) on to the glass slab on which the paper points were laid, and it foamed up in five minutes to the size of half a chicken's egg. The patient then had relief, and we applied a dressing of pure beechwood creosote and glycerine, one of creosote to three of glycerine, and sealed it with gutta-percha. Next day the tension was less, but we drained out almost as much pus as there was at the previous visit. Another dressing of creosote and glycerine was applied and left for two days; about half the amount of the original quantity of pus was drained out, and the patient had no great pain. This treatment was continued for about ten days, which included three dressings, and then, when pus had practically subsided, we applied two subsequent dressings of mummifying paste on bibulous paper to the canal. Although pus and pain had quite ceased, there was considerable induration from the severe invasion of inflammation of the apical and labial tissues. The root was finally filled with the author's medicated root filling, which is described under the second division of this chapter. The point we wish to accentuate in the description of these cases is the nature of the anatomy of the apical foramina of a root canal, which we have shown to have no definite relation to the axis of the canal, and although we must not perforate the side of a root with a drill, yet we must not leave an abscess to itself. If there is a known or a suspected curve in the shape of the root, and there is little chance of negotiating it, we must abandon the method and do what we decide is best for the case. But this forms the subject matter for the latter part of this chapter.

Dr. Callahan and others have demonstrated the existence of the multiple foramina of roots, and they may also be examined under the microscope. We must respect the apical end of a tooth as much as we do the coronal end, and realize that there are several foramina to deal with, and it is as unreasonable to neglect these as it would be to treat one root canal and leave another one belonging to the same tooth.

While discussing the anatomy of the pulp we must also realize the existence of the periodontal membrane, outside the 
root, which lines the alveolus. If any untreated foramina contain débris from the tiny vessels which pass through them, our acquaintance with the microscope teaches us that they are large enough to harbour micro-organisms which will infect the periodontal membrane, and set up inflammation with abscessed conditions. We all get our failures, but many of them can be avoided by a comprehensive acquaintance of the delicate nature of the apical tissues outside the root of a tooth. Again, there are operators who overlook the delicate nature of the dental periosteum, and keep on applying irritant drugs for maintaining rigorous asepsis. It is important, of course, to destroy microorganisms; but if drugs and medicaments are not intelligently and patiently applied, they will destroy the periodontal membrane and the bone too; if not directly, they will bring about the gradual degeneration and disintegration of these tissues. The preservation of the activity of the periodontal membrane is the primary object of root treatment, and we must neglect no point which will help us to secure its healthy condition and co-operation; it is the supreme factor in successful root treatment and filling.

Arsenic or arsenious trioxide is employed for the purpose of devitalizing the dental pulp. The destruction of the vessels of this organ is brought about by corrosive action on the capillary bloodvessels causing a general hæmorrhage, followed by thrombosis of the blood. The physiological action of arsenic has been investigated by many men, which has given rise to many theories, but they all appear to agree that the final consequence of its action is to cause a capillary hæmorrhage and embolism of the blood in the vessels. If a pulp taken from a tooth which has been extracted on account of severe pulpitis, is flattened and examined under the microscope, the bloodvessels, which are almost transparent in a normal pulp, will be found to be a dark brown colour, and look like a large tree with creepers intertwined, showing the amount of congested blood that will engorge the vessels when inflammation is present. If we take an orangewood stick, sharpen it to a fine point, place it against a live pulp, and then deliver a smart blow with a mouth mallet, it will bring about a similar condition to the action of arsenic, inasmuch as when the walls of the bloodvessels are punctured throughout its mass, hæmorrhage occurs, which disorganizes the whole organ. This method, of course, is crude, but the writer had a personal experience of its efficacy many years ago, when it was 
applied to one of his own teeth, and although he would not like to submit one of his own patients to it, yet it proved conclusively that the pulp was incapable of acting as a sensory organ. It was removed and the canal drilled out and dressed at the same sitting. When we apply pulp pressure anæsthesia or administer $\mathrm{N}_{2} \mathrm{O}$ gas the same result may be brought about by passing a fine nerve drill or a pulp extirpator up the canal to the end. When a pulp dies after prolonged and painful pulpitis, the hyperæmia which accompanies it causes strangulation of the vessels by internal congestion and pressure against the apex of the canal. There appears, however, to be some difference between the action of arsenic, and also the spontaneous death of the pulp under pulpitis, and the direct mechanical destruction of the vessels. In the first two cases devitalization is complete, but with mechanical destruction, if a piece of pulp tissue is left behind it will sometimes give a lot of trouble, as its vitality seems hypersensitive, which rather points to the conclusion that strangulation of the vessels through engorgement takes place from the apical end.

Although the use of arsenic is the principal'means for bringing about devitalization of the pulp, some operators who are nervous about it prefer the use of cobalt, which contains arsenic as an impurity. Cobalt is found in proximity with arsenic in nature, and it is very difficult to obtain it from a manufacturing chemist free from arsenic. This shows what a small amount of arsenic is required to devitalize the pulp. Most authorities speak highly of arsenic as a devitalizing agent for this purpose. It may not be as quick or as direct as mechanical destruction under an anæsthetic, but it is more definite in its results if applied with a knowledge of its potency.

Pressure anæsthesia is employed for devitalization by taking a tiny crystal of cocaine or a larger one of novocain and moistening it with a drop of adrenalin ( $\mathrm{I}$ in $\mathrm{I}, \mathrm{OOO}$ ). A small piece of amadou or cotton-wool is saturated with the mixture and placed in position over the well-exposed pulp with a piece of unvulcanized rubber covered over it. Then pressure is gently applied with a large, flat-surfaced or an oval plugger or burnisher, watching the patient's face for any sign of pain through too much pressure. This is kept up until the tooth does not respond to further pressure. We then take a pulp extractor, pass it up the canal to the apex, and twist it to allow the barbs to entangle 
the pulp tissue, and then withdraw it. This is a very convenient method of doing the operation quickly and at one sitting, but it is open to objections. Reference to Fig. 43 and Fig. 92 will show that some teeth are abnormally long, while others are unduly short. The pressure used and the time allowed would probably fail to reach the apical portion in the cases of long teeth, and we may be faced with an obstinate and super-sensitive pulp end, which might be left behind under the root filling, the operator thinking he had reached the apex with his nerve

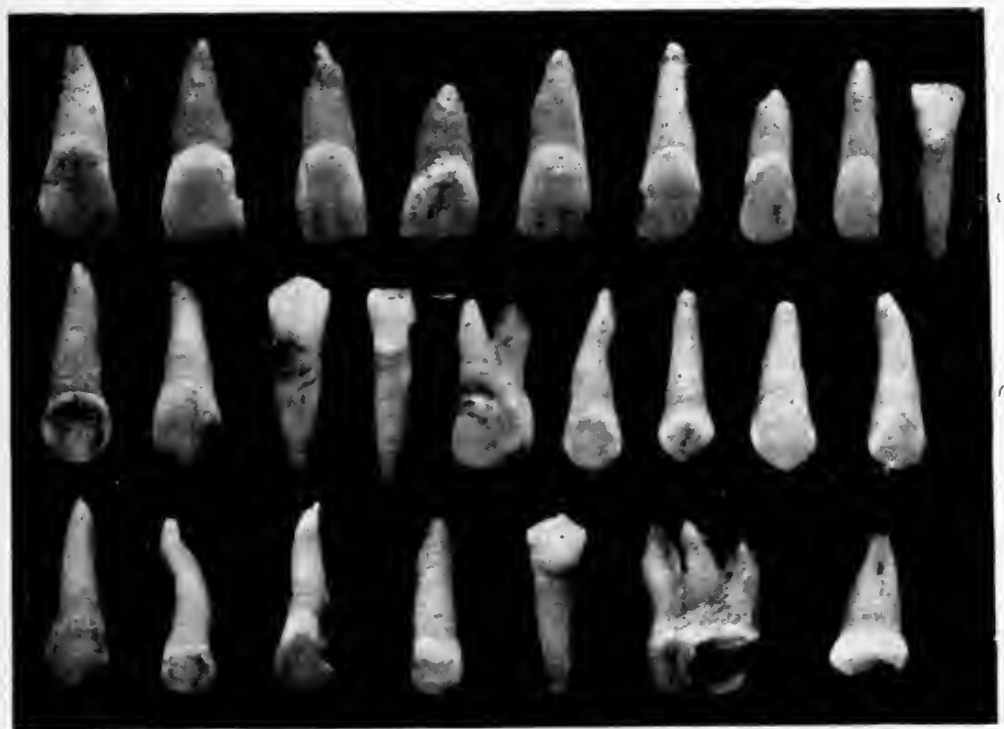

Fig. 92.-Examples of Undersized Roots.

broach, and if left without mummifying treatment would probably cause septic trouble. A 50 per cent. solution of pure sulphuric acid is considered the best application for this condition. In the case of short rooted teeth we shall probably induce anæsthesia beyond the apical area, and there is risk in some canals of drilling beyond the apex, as there is no sign of pain to guide the operator, thereby setting up inflammation in the bone. Furthermore, the hæmorrhage which occurs in this method precludes satisfactory filling of the canal at the same sitting. The method, while being useful, needs great care. The same remarks apply to extirpating the pulp while the 
patient is under $\mathrm{N}_{2} \mathrm{O}$ gas. We are all glad to adopt it at times, but arsenic gives the best results.

The application of arsenic requires intelligent manipulation It must not be applied to a severely aching pulp straight away, as the hyperæmia of the bloodvessels in pulpitis resists the absorption of the arsenic, which so irritates the nerves that the pain becomes intolerable and can be as intense as having the tooth extracted, and lasting for some hours. The hyperæmia must be reduced first. A dressing of Buckley's Phenol or Dentalone applied alone or mixed with a little tannin will usually be successful if sealed in carefully for twenty-four hours. This preparatory dressing must be sealed in without any pressure; a piece of card as a cap will protect it while placing gutta-percha over it. The dose of arsenic or arsenic trioxide is from ${ }_{16}^{1}$ to ${ }_{60}^{1}$ grain. We cannot fix the amount by weight in practice. Small pellets and discs are sold which contain a given dose, but there are various compounds of arsenic, with and without anodynes, on the market, also a useful form known as S. S. White's Devitalizing Fibre, which has been in popular use for many years. If arsenic alone is used, it should be moistened with carbolic acid or creosote, and the tiniest spot, equal to the point only of a small pin, applied on amadou or cotton-wool should be placed in direct apposition with the exposed pulp. A trace of Buckley's Phenol, or any soothing dressing, may be put on the wool to assist the arsenical action in penetrating quicker. The pulp should be exposed as completely as possible, as pain usually develops if proper contact has not been secured. No pressure whatever must be caused by sealing it in, neither must there be any possibility of the arsenic leaking out. Oxyphosphate of zinc or temporary cement may be used, which is the surest way of sealing it in and protecting the pulp from pressure. Care must be taken to see that the seal reaches the cervical edge in proximal cavities. This appears difficult to beginners, and the arsenic dressing is apt also to become lost in the cement. Some operators apply a little cement on a piece of tin or paper and carry it to the cervical part first, then apply the arsenic dressing and cover it with more cement. Guttapercha can be used as a seal, but if the dressing is not protected by a stiff cap there is risk of pressure causing pain when biting upon it.

Arsenic should not be applied to children's temporary teeth or any form of root treatment which involves going down the 
roots. „Some operators apply palliative dressings in these cases. Neither should arsenic be applied to children's permanent teeth where completion of the roots has not taken place (see Periods of Dentition on p. 36, and also Fig. I2), as there is danger of its being rapidly absorbed and carried out into the apical tissues through the open ends and setting up inflammatory trouble and even necrosis.

The time allowed for arsenic to complete its work is usually twenty-four hours, but we prefer to use as small a dose as possible, which can only be determined by experience and leaving it in, according to the estimated size of the pulp, for three or four days. If a relatively large dose is used, there is risk of the tooth becoming sore and painful through periodontitis.

Sometimes the membrane of the pulp is hypersensitive when we remove the arsenic dressing. If this occurs it does not inean that we must necessarily apply another dose of arsenic. Matters may be worse if we do that without sufficient reason. The point is that we must try to get through the membrane by using a cross-cut bur or a nerve broach dipped in creosote, tricresol, or a moistened crystal of paramonochlorphenol placed on the part. When through the membrane we usually tind in these cases that the arsenic has done its work. If the pulp is still found to be painful, a second application of arsenic may be necessary.

Tne next important point is that when the arsenic dressing has been removed, the pulp comes away piecemeal and with a certain amount of bleeding. This is an unsatisfactory condition in wnich to till the root canal. Tne better way is to apply an astringent dressing such as Buckley's Phenol, Dentalone, creosote, and glycerine, mixed with tannin to a thickish paste, and applite on bibulous paper and left in for a couple of days. Upon removal of this dressing we must open out the orinces of the canals (if in a molar or bicuspid). It is better to go directly for these and ignore the pulp chamber, as unnecessary burring destroys the gutters which help to conduct our nerve canal broacnes to them, and also in discovering any additional canals that may be present.

After removal of the pulp from the canals, as far as is necessary, we may fill them with thickly mixed mummifying paste, which is now regarded as legitimate practice. Mummifying pastes, however, are abused, and cannot be considered as being 
perfect. Their use involves a valuable principle, and can certainly be utilized subject to certain considerations. If the canals are fairly easy to get at, the pulp tissue is cleared from them as much as possible with a fine, long excavator or a pulp cleanser, and then filled with mummifying paste and capped over neatly with oxyphosphate of zinc cement and the cavity filled with the permanent material. But if we desire to fill the roots properly we must open them up in a direct line with the axis of the canal (see Figs. 93 and 94). The instrumentation of opening up canals is a very important matter. We have already observed that the pulp chamber and canals are situated within the crown and roots, therefore it is not necessary with freshly devitalized pulps to open the so-called apical foramen; there is a piece of tissue there, but even if we could remove it with certainty there are probably other foramina so situated that it is impossible to get at them. Therefore we remove the whole of the pulp from the canals. In order to do this properly and apply the root-filling we must get at the parts in an open line and not attempt to sneak into the canals from an angle, which often causes other trouble, such as losing our way and drilling out at the side of the root. The periodontal membrane will not tolerate injury of this kind, but there is a high degree of toleration at the apical end of the root.

The problem before us, therefore, is how to get at the canals and define them in order to open them up without losing our way. This is an important stage, and needs close observation to become experienced in the diversity of the forms of canals. In conjunction with early difficulties every opportunity should be embraced to hold a post-mortem examination on every tooth that we can find. From the point of view of our subject it is wasting an opportunity to throw an extracted tooth away without examination. An extracted tooth should be made responsible, as it were, for the successful retention of at least one lame tooth in somebody else's mouth. An examination of the few teeth illustrated in this book shows what a diversity there is of sizes and shapes of roots, externally; also the lack of corresponding relation to the diameter, shape, and position of the canals (see Fig. 93). These variations are endless, and we cannot be skilful with our delicate instrumentation if we are not equally familiar with the endless variety of root canals which we have emphasized. 

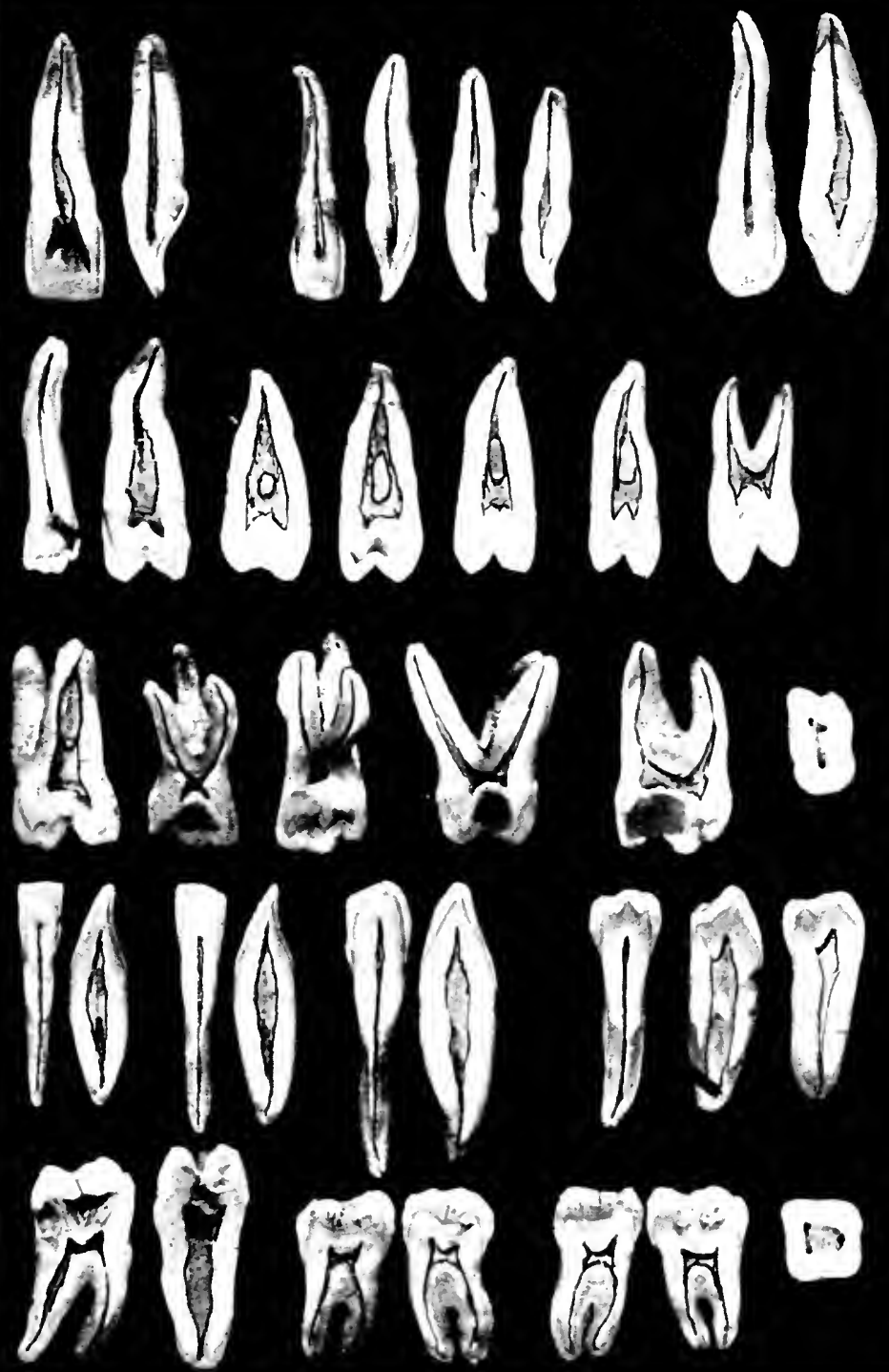

Fig. 93.-Specimexs of Dissected TeEth, Showixg Ramifications of THE PILP.

They bhave not been indiscriminately cut, but carefully ground out on the lathe, as will be seen by close observation of each one. The top three rows are upper teeth; the two bottom rows are lower teeth.

This illustration may be studied in conjunction with Fig. 94. 
Let us examine the various teeth with their roots. The upper central canal is somewhat flattened on the sides and is easily penetrated with broaches and drills. The upper lateral canal is also flattened on its sides, but is not so easily penetrated, and is sometimes difficult to drill successfully; much care is needed to open it up. The upper canine canal is large, flattened at the sides, with plenty of room for the penetration of broaches and drills. The point to be careful about with this tooth is its length, and to see that we really get to the end of the canal, if possible, as it often has a curve near the apex. In order to locate the canal in any of the upper front teeth from canine to canine, we commence with a very small coarse carbo-wheelstone and cut through the enamel just above the cingulum on the lingual side. Then take a round bur and bore at an angle of 45 degrees to the axis of the tooth, which will then expose the horn of the pulp. We must now be careful not to use any instrument which will make a ledge anywhere, as a subsequent drill may rest upon it and start a false canal, which causes much annoyance and waste of time. Having found the pulp chamber we take a long root bur, which is really a Humby's root roughener about twice its length, flame-shaped, and made in three sizes or thicknesses. These burs have a blunt point on each one which will follow the orifice of the canal, but cuts only on the sides. The point of the bur is kept in the canal, while with a sawing motion we extend the first hole we made until its position is in a direct line with the axis of the canal. If we wish to preserve the crown of the tooth and fill it we should not make the groove quite in the axis line, but if we have to treat an abscess, we must do so. We then enlarge the pulpal chamber part of the canal with a Gates-Gliddon drill dipped in bur lubricant, and pass a pulp cleanser broach right up to the end of the canal and draw the pulp out, which, after the application of the tannin dressing, is tough and in a dried-up condition. In using all cutting instruments for canal work we use the bur lubricant because it facilitates the process and prevents them getting hot and breaking; it should be kept separate for this purpose. For this work it may be prepared with tricresol (see Fig. 94).

With upper biscuspids we find opening of the canals somewhat more difficult. The upper first bicuspid is usually tworooted, or bifurcated, and the second one single-rooted with 

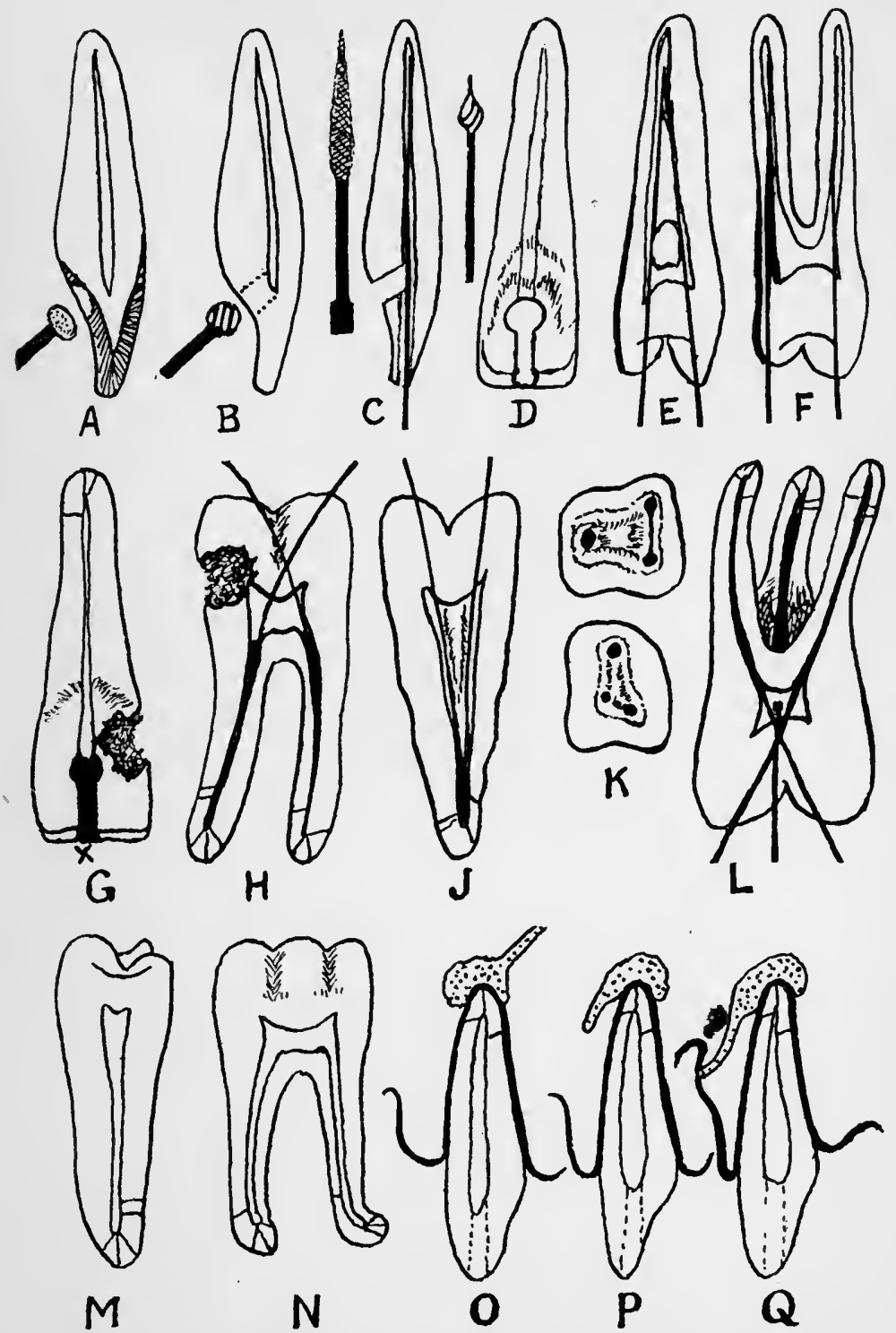

Fig. 94.-Diagram to show Principles of Opening up Canals.

$A$ to $\mathrm{D}$ and $\mathrm{G}$, opening up upper or lower front teeth; $\mathrm{E}$ and $\mathrm{F}$, upper bicuspids; $\mathrm{H}$ to $\mathrm{L}$, opening up lower and upper molars; $\mathrm{M}$ and $\mathrm{N}$, showing foramina at apical ends of roots; $\mathrm{O}, \mathrm{P}, \mathrm{Q}$, abscess sacs showing opposite directions of a fistulous tract. 
a very flattened canal. Care is needed to avoid drilling between the two roots. We can usually tell where we are by making a rule to open the labial and lingual walls first, and pass a broach up on one side and another on the other side. If the broaches widen apart outside the tooth, and incline towards each other in the canals, it indicates that they run to one apex, whereas if the broaches incline to the opposite angle or are parallel it indicates two roots or a bifurcation.

Upper six-year-old molars, and also twelve-year ones, require special study, as to the relation of the canals to the outside configuration of the tooth. The pulp chamber is rather small, and the buccal posterior root is difficult to find very often. The position of its entrance to the pulp chamber is apparently out of relation to the external position of the root. If we examine this root out of the mouth we shall find that this has the appearance of having been joined on like a branch. When this has been observed we can better understand where the canal is likely to be. Some operators bur the interior of the crown and the pulp chamber too much, which weakens it unnecessarily. The pulp chamber being small, therefore the three canals are near each other, and if three broaches are placed in them, they will be found to cross each other at a point approximating to the occlusal surface, so that all three canals may be reached theoretically and often practically from a hole bored right in the centre of the crown. When mummifying paste is used for filling, the buccal root need not be defined deeply, as the pulpal tail is very fine and can safely be left to the action of the paste. The buccal anterior root should be opened as far as is practicable, but the palatine canal is usually very open and should be filled right up to the apex.

The wisdom tooth canals may be found either to be similar to the other molars, or to have a larger pulp chamber and one common canal.

The lower incisors require delicate manipulation as the canals are very flattened and fine in their bore from side to side, which makes it a difficult matter to penetrate. The outer surfaces of their roots are very flattened on the sides, which shows that they may easily be perforated from within.

Lower canines are similar to upper ones, but flatter at the sides.

The bicuspids are straightforward, especially the second ones. 
The lower molars require care. If we examine a six-year-old one we shall find that the anterior root is quite different from the posterior one. The anterior root has a very flattened canal from the lingual to the buccal side, but at each extremity it has a fine open channel which may be penetrated. The two channels usually meet at the apical end. If we attempt to open it in the centre we shall very probably perforate the side of the root under the crown. On the external surface of the root, just

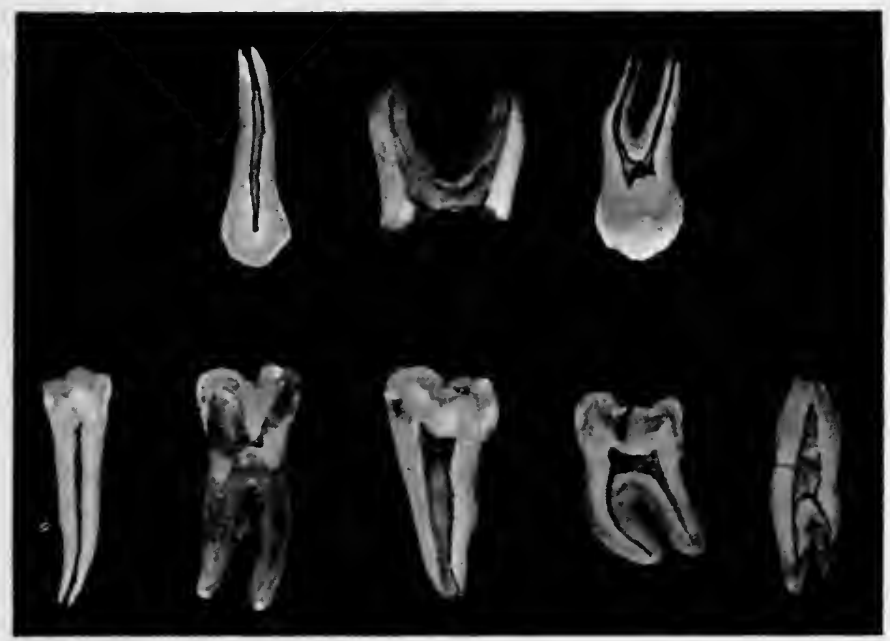

Fig. 95--Specimens showing Conditions of Some Canals UNFAVOURABLE FOR ROOT FILLING.

The molar root in centre of top line contains a completely calcified pulp. The left lower molar in bottom line has canals and pulp chamber completely filled up with secondary calcification. The end lower canine has two roots.

below the bifurcation under the crown, there is a depression which makes that wall of the canal exceedingly thin. Therefore we treat this canal as being two canals, and open them well each side before passing a broach or drill down. The posterior root is well defined and is easily penetrated, excepting that all molar roots are prone to curvature.

Some canals give us some trouble in defining and opening them up owing to calcareous deposits from the pulp. They are often only local, and when the obstruction has been negotiated the rest of the canal may be quite open. These calcareous deposits 
sometimes extend for a distance, and even the whole pulp may become calcified (see Fig. 95).

The selection of a suitable root filling now arises. For molars and bicuspids the condensation of thickly mixed mummifying paste will answer the purpose if applied as we have already indicated. We have experimented with various materials for many years, and have succeeded in producing two that have given us every satisfaction. These may be used in combination, or one root filled with one kind according to our judgment, and another root of the same tooth filled with another kind. We have found that all root fillings should be medicated in some way or other. Even when crowns with posts are cemented in roots the apical ends must be medicated in order to leave the foramina secure against septic trouble (see chapter on Crown Work).

Condensation is as necessary in root filling as with cavity plugging. This does not mean that we must force material beyond the root, because that is wrong in principle. In order to secure this we may use gutta-percha canal points with the ends cut off and plunge them with moderate pressure into place.

Formula for mummifying paste (Dr. Soderberg's):

$$
\text { Powder }\left\{\begin{array}{lll}
\text { Pure zinc oxide } & \ldots & \ldots \\
\text { Thymol crystals } & \ldots & \ldots \\
\text { Calcined alum } & \ldots & \ldots
\end{array}\right\} \text { Equal parts by weight. }
$$

Liquid: Pure glycerine.

Mix powder and liquid in the same way as a cement, but very thickly as a root filling. Plug with nerve canal pluggers. A practical set may be made up by selecting long probes of varying thickness and cutting off the sharp ends, and cavity pluggers with a flat surfaced end. Instruments with ball ends plunge into the soft filling, and counteract condensation by either pulling it away upon withdrawal or rendering it tenuous. For plugging the apical portion a very fine carrier is employed which will allow of the filling material being carried right up to where it is required and allowing air to be driven out. There is nothing better for this purpose than a fine pulp cleanser with the barb end cut right off and fixed into a broach holder. When the mummifying paste is in position take a piece of amadou and press it well over it, leave it for a minute, take it away, then trim the edges and neatly cover it with a box-shaped cap of oxyphosphate of zinc. Fill the cavity with amalgam or whatever is considered suitable to the case in hand. 
The root filling cements which we use in addition to the above are (the author's formulæ):

\section{No. I. Root Filling.}

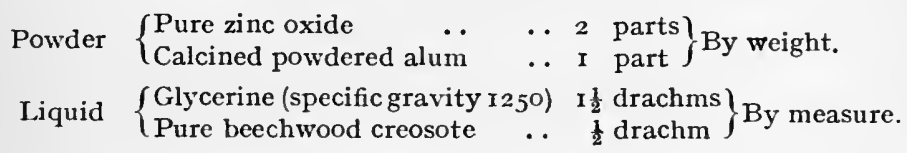

Mix like a cement to a thick consistency and fill the canals. After a few hours it will set. If obtainable, calcined oxide of zinc is preferable to the ordinary form.

\section{No. II. Root Filing.}

To prepare this we must purchase a packet of oxyphosphate of zinc cement, which is used for filling cavities. Colour: white -usually slightly ivory, which is quite all right, but if yellow it should not be used, as the colouring ingredient is usually sulphide of cadmium, which is better left out for this purpose.

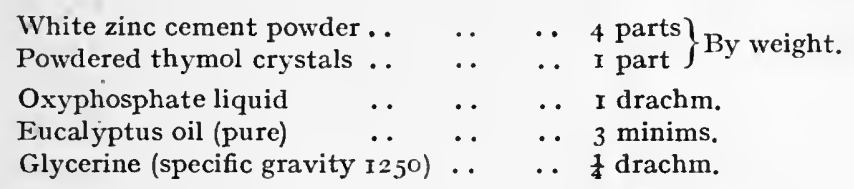

Mix as a cement to a moderate consistency, and fill the canals. It works beautifully with a creamy flow, and sets quite hard. The construction of this formula is based upon the chemical compatibility of the various ingredients. It is non-irritant, has suitable working qualities, and sets hard. Its degree of medication is sufficient.

The canals are cleaned out with absolute alcohol applied with a square, soft canal broach, which has a wisp of Japanese bibulous paper caught at one end and twisted around it. We hold the piece of paper between the thumb and forefinger of one hand and the square broach with the other. thumb and finger, and lay the point of the broach upon the paper, then pinch the paper and twist the broach, when it becomes securely fastened on to it. For employing medicaments we apply them in the same way, but not quite so tightly wrapped. This enables us to carry the medicine to the apex where it is required without squeezing it out in its passage up the canal. By turning the broach in the reverse direction we loosen it and bring it away 
while leaving the dressing behind. Care is required in applying dressings on cotton-wool, as they are apt to become jammed in the canal and are difficult to remove.

2. The second part of root treatment consists of what might be called advanced root treatment. The tooth in this case is dead when we are called upon to examine it, or it may be accompanied by a large swelling of the tissues round the apical area, or there may be a fistulous opening on the gum either lingually or labially with pus oozing from it. Dead teeth which give no indication of abscess trouble must be treated as though they were septic, and if there is no septic complication the sooner will the tooth be ready for filling. Unfortunately, many apparently harmless dead teeth prove to require serious treatment, so that we must not be misled by apparent absence of inflammatory symptoms. Even if a dead tooth is apparently harmless, yet the canal of the root being full of débris must be submitted to the same precautions and care as if it were septically active. The treatment of dead teeth with abscess conditions is rightly regarded as a difficult operation, but as it involves skill of a high order and ensures a patient's gratitude when successfully accomplished, renders it a sufficient inducement for the operator to become competent in its practice.

It must be understood that only an apical abscess can be dealt with. This condition can usually be diagnosed by seeing the external tissues highly inflamed, or a swelling at the apical region accompanied by extreme tenderness on pressure being applied, or the tooth feels very tender to bite on, caused through the periodontal membrane being highly inflamed. Any of these symptoms may be accompanied by the existence of an abscess sac which for the time being may, or may not, be discovered.

The object of the first part of root treatment was the whole of the pulpal area inside the tooth, but in advanced root treatment our object is to deal with diseased conditions outside the tooth involving the immediate apical region of the tissues. In order to carry it out successfully we must insist on the faithful observance of five principles:

r. Our object must be to reach the apex of the canal and just through it.

2. We must sterilize the column of putrescent débris contained in the canal to prevent infection of the periodontal membrane from it. 
3. We must secure drainage by instrumentation.

4. We must apply suitable medication or drug treatment to heal the abscess and render the whole area aseptic.

5. Root filling well condensed.

All these principles must be carried out in each case if success is to be attained.

I. If we cannot carry out the first principle we may as well abandon our endeavours altogether. There are some roots which render it impossible to reach the apex, such as the buccal roots of upper molars and the anterior root of a lower molar, upper laterals, and lower incisors. Upper centrals, canines, and bicuspids, lower canines and bicuspids, usually have good canals which enable us to carry this principle into effect.

2. If a root has not infected the dental periosteum we must be careful to see that we do not disturb its putrescent contents until it has been sterilized by suitable medicaments. Or if an abscess is present we must not force more septic débris into it. The use of pure formaldehyde solution or formalin, with tricresol, equal parts, as recommended by Dr. Buckley, or formalin and creosote, equal parts, which may be introduced on cottonwool in a carefully prepared enlargement of the orifice of the canal and sealed in with cement to retain the formaldehyde gas which is developed by the warmth of the mouth, will sterilize the column of débris and neutralize the irritating gases, ammonia, and sulphide of hydrogen, which are the products of putrescence. Experience has shown that these strong percentages of formalin should not be applied near the apical tissues, but they are useful for this purpose only.

3. The principle of securing drainage is most important. We regard efficient instrumentation and medication as being the foundation upon which advanced root treatment rests. Disregard or modification of its requirements spells failure straight away. The more general use of radiography will undoubtedly be a valuable adjunct to treatment, as by a correct reading of radiographs granulomatous areas and abscess sacs may be discovered. The size and direction of roots by its aid can be revealed, which will make instrumentation easier. To secure a passage for the drainage of an abscess there must therefore be a carefully carried out process. We must enlarge the canals sufficiently to allow paper points to pass up and syphon out any pus that may be present, and subsequently to 
pass up a suitable medicament for healing the inflamed tissues. The opening of the foramen at the apical end of the root must not be large, but only sufficient to allow the passage of the fine paper points. We open out the mouths of the canals with root burs, then follow on with Gates-Gliddon drills of suitable sizes and complete the apical portion with Beutelrock's, Kerr's, or other similarly shaped nerve canal drills, care being taken not to drill through the sides of the canals or to penetrate the apex farther than the external surface. The paper points may pass up as high as the space of the abscess will allow. Root drill lubricant is used for all cutting burs and drills in order to facilitate cutting and to avoid blocking up with hot débris which becomes hard, and to prevent fine drills from breaking. Drills should be withdrawn now and again to allow of clearance of the débris. An experienced root filler can tell intuitively when he has pierced the apical end. Practice out of the mouth is time well spent. If there is an abscess sac, a broach will sometimes go some distance beyond the apex, but if there is no sac but inflammation of the dental periosteum, the instruments must not inflict any damage on it. An active abscess sac will probably require one dozen to eightcen paper points to empty it at the first application, after thoroughly sterilizing the canal by previous dressing as explained under principle 2. A dressing containing a suitable drug is then sealed in and left for a day or more, according to the severity of the case. Drainage is again carried out, and a dressing applied until the discharge has ceased and all tenderness on external pressure has disappeared. The operator should note how many paper points were used on each occasion. When treatment is completed there will always be a slight moisture on the extreme end of the paper point.

We sometimes find that an apical abscess will give us a lot of trouble, and it is as well for us to realize the nature of the forms which the sacs take in their development. A brief outline of the stages of disease, and their final consequences to a tooth, may be stated as follows: The mouth may be compared to a pond teeming with organisms which thrive on its bed and banks. The oral cavity provides suitable accommodation for bacteria consisting of pathogenic or disease-producing types, and non-pathogenic types, which are harmless under ordinary conditions. If a suitable portion of the mouth 
around the teeth, such as the interdental spaces or a ledge or crevice on the tooth-surfaces, becomes prepared through lack of cleanliness, certain micro-organisms form a colony and enclose themselves with a formation of gelatinous plaques under which lactic acid is produced sufficiently concentrated to attack and dissolve the lime salts of the enamel. They then work upon the dentine until the calcium salts have been removed and the soft, decalcified tissue is exposed. Some micro-organisms have the power of peptonizing or dissolving this until it is broken down into débris. When the pulp has been disorganized it presents a menace to the periodontal membrane, which at some time or other becomes inoculated with septic matter from the putrescence of the canal. An abscess sac is formed which at first may be very tiny, but may develop into a large one with considerable swelling extending into the surrounding bone and external soft tissues. As the internal pressure develops it seeks a way out in order to empty itself. A fistulous tract or canal is formed which usually takes the line of least resistance. It finally ends with an outlet which is commonly on the external apical surface of the gum against the tooth which is the original cause of it; but it does not always do this, and that is where trouble is likely to become serious to the patient. The fistulous canal and opening may take another direction. It may travel along the external surface of the tissues and break some way off from the original tooth; or along the palate, breaking in the centre of the vault; or farther along into the soft palate, emptying into the throat, where the pus will weep out and get into the larynx, thereby infecting the lungs and setting up septic pneumonia; or it may break into the floor of the antrum and set up an empyema; or travel up the facial tissues or into the nasal cavity; or, again, along the neighbourhood of the inferior dental canal and break into the throat. The operator must be prepared to act as his judgment and experience guide him. If the fistulous opening is on the gum, somewhere near the tooth being treated, it is the easiest form of abscess sac to deal with, as we know the extent of it, and can pump medicaments through with a fair chance of stopping the discharge.

4. Suitable medication is an important factor in itself. It is remarkable how some operators expect a cure-all drug applicable to every case. We may all have a routine agent or two for straightforward cases, but if our intention is to face diffi- 
culties and overcome them, we need a selection of drugs as our root-treatment armamentarium.

The dental profession is indebted to such men as Buckley, Callahan, Tomes, Miller, Black, Prinz, and Crane, for their investigations into the nature and action of drugs suitable for root treatment and filling. Their combined laborious work cannot have been in any way simple, but their labours have certainly simplified many obscure problems and made the subject capable of being better understood.

The utilization of formaldehyde vapour for disinfection of the canal and dentinal tubuli, and for neutralizing the irritant gases from putrescence, as demonstrated by Dr. Buckley, is an important bactericidal remedy, but it must be applied with prudence. A 50 per cent. formalin mixture with tricresol or creosote is good for the initial dressing at the mouth of the canal, but this agent is too irritating for application near the apex. It has a marked preservative and mummifying action upon dead tissue, but this is not desirable where vitality is required. A 3 per cent. solution is quite strong enough, and should not be forced through the apex; this strength can be added to mummifying paste and incorporated well into the powder. A non-irritating dressing for putrescent conditions is Black's I-2-3, which is a mixture of carbolic crystals, I part, oil of cassia, 2 parts, and oil of wintergreen, 3 parts. Oil of cinnamon is a powerful and penetrating bactericide, but must be kept under control, if used pure, because if it should get out at the apex it is too irritating, and will quickly produce the symptoms of inflammation which we are endeavouring to prevent. It may be mixed with glycerine in varying proportions, or used as a dressing mixed with zinc oxide, which will prevent any free oil escaping; or it may be applied on a wisp of non-absorbent cotton-wool and partially dried before insertion. Cinnamon oil is valuable because it contains cinnamic aldehyde. Oil of geranium must be controlled in a similar way. Thymol is regarded as quite a dental medicament, and is a valuable one owing to its bactericidal qualities and low degree of toxicity. It is very irritating when applied in a concentrated form in a mixture, but is capable of exercising its potency in weak solutions. It may be added to glycerine, I in 200 parts. One grain of alum, which has a mild astringent effect, may be added. 
Sometimes we have a case where there is no sign of pus, but the periodontal membrane at the apex of the root is exceedingly painful, and the drugs which we apply to ordinary cases make matters worse. In these cases palliative dressings are, of course, needed. Paramonochlorphenol, methyl-salicylate with 5 per cent. carbolic acid crystals, and creosote in weak solution with glycerine, will usually give relief.

With cases where there is a fistulous tract and opening and the pus is difficult to stop we can pump a medicament right through it. This is carried out by using the root canal as a syringe barrel and a canal plugger or a broach as the plunger. The broach is warmed and white wax is run round slightly and bibulous paper wrapped round. It is then dipped into one of the following mixtures and worked into the canal patiently with a pumping movement until we see the solution appearing at the opening. At a subsequent sitting we may wish to repeat the operation, but find that the opening has closed; we can, however, reopen it with a sterile ball probe and proceed as before. The suitable mixtures are: Camphenol, 2 per cent. in glycerine; creosote in glycerine, I part to 7 or stronger; and for stubborn cases, phenol-sulphonic acid (see paragraph on p. I87).

The application of a counter-irritant to the external surface of the gum is sometimes useful, such as tincture of aconite and liniment of iodine mixed together, or the use of dental plasters.

Internal remedies will often help matters, such as an antibilious pill to regulate the liver-it need not purge and can be repeated the following night; aspirin, dose 5 to $5_{5}$ grains; or the patient's medical man can administer a course of tonic or other treatment in order to assist the resistance of the blood.

The recuperative powers of the patient is a factor which must not be overlooked, and root treatment should be withheld in many conditions of a patient's health, because, after all is said and done, our endeavours are only means to help the tissues to recover and to retain their health and vitality.

In some very obstinate cases the continued flow of pus has shown no permanent sign of stopping. These cases have been marked by a mixture of blood and pus. After three or four months' treatment we have had to give them up and extract the teeth, when we have found in the majority of cases that the ends of the roots were roughened and partially absorbed. This necrosed condition is probably caused by the acid reaction of 
a long-standing septic condition. In other similar cases where the patient has expressed a wish to still retain the tooth, as no pain was present, we have plugged the canal with a temporary root filling of mummifying paste applied on bibulous paper and left in with the understanding that if it gave any pain or trouble at all the tooth could then be extracted. To our surprise, in some of the cases, we have opened the canals six months or a year afterwards and found that the obstinate discharge of pus had stopped entirely. These cases have demonstrated that rest between final dressings is a useful factor.

5. The canals may be filled with our formulæ I. or II.; or with Eucapercha, which is a mixture of:

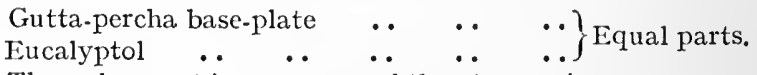

Thymol, I part in 200 parts of the above mixture.

Oxy-chloride of zinc is favoured by some operators.

There is no royal road to root treatment save by practice, observation, and experience, which may be stimulated by our personal interest, and our patient's appreciation. 


\section{CHAPTER XXI}

\section{CROWN AND BRIDGE WORK}

THE operations which are carried out in the dental surgery include crown work. The assemblage of a combination of two or more crowns as piers or abutments is called "bridgework." This combination of crowns and abutments is capable of an endless variety. The form of substituting artificial crowns in place of broken-down or missing natural ones is most enticing to patients who have a reluctance or prejudice to wearing a plate or denture; consequently, they are most willing to have this kind of work fitted into their mouths. We refer to fixed bridgework, which is cemented into the patient's mouth and cannot be removed for cleansing purposes, but is cleaned in the same way as their natural teeth. One can quite understand a patient's preference for a bridge which keeps the palate and tongue free from encumbrance. It appeals to them as being highly skilled and artistic work. But in this case we often find that the old proverb "that chickens will come home to roost" is applicable. Crowns and bridgework have been put into mouths which are not suitable for their reception and permanent wear, with the result that many medical men are now condemning them as being traps for septic matter and do not hesitate to order their removal. So strong is their conviction on this point that they will order their removal on the slightest suspicion of periodontal trouble. Unfortunately, the charge of abuse against this form of work is not ill-founded. and the grounds of condemnation may be thus summarized: badly fitting crowns and abutments cause leakage, which allows lactic and other acids to concentrate on the dentine at the necks of the teeth and destroy them; food collecting against dummy crowns causing hyperæmia of the gums, which then become foul and septic; badly fixed bridgework through imperfectly mixed cement, and set without securing a proper degree of dryness; utilizing dead teeth for abutments, especially 
where the fixed position of a number of dummy crowns causes too much strain on the periodontal membrane of the socket, the vitality of which has already been lowered. Hyperæmic and septic conditions of the mouth always become exaggerated where bridgework is fixed. The same objections apply to single crowns, especially if there are several of them in the mouth, but they are not so serious from an inflammatory standpoint providing that they are well executed in every detail. Shell crowns, however, must be included with the same objections as fixed bridgework. The main point to be considered when estimating the value of this kind of work is the standard of quality of the operator's work. From an operative and pathological point of view even the best of well-fitted collars and joints transgress the laws concerning the accuracy of flush surfaces around the cervical area of a filling.

With the reservations and objections to which we have referred there are many cases where crowns and bridgework may be fitted and do good service to the patient, but attenticn to details is imperative. It is impossible to describe this subject in one chapter. The writer has had thirty years' strenuous experience with this class of work, and can speak with practical knowledge of its primary principles:

I. The preparation of teeth with living pulps.

2 . The preparation of teeth with dead and septic roots.

3. The fitting of an all porcelain or a porcelain-faced crown.

4. Shell crowns.

5. The stages of making fixed bridgework.

6. The advantages of removable bridgework.

The first two principles have been described under Root Treatment and Filling, but there are technical points adjustable to crown work, but no alteration of pathological principles.

Porcelain is universally admitted to be the most desirable material for the restoration of the missing portion of a tooth. Porcelain inlays involve an unreasonable amount of time in a busy practice, but the fitting of a porcelain crown is as near perfection in our art as can be expected. It involves a lot of attention to details, but when completed well repays the operator for his pains.

We will now consider the fitting of a central or a canine crown. The patient presents the tooth, which either has to 
be devitalized or root treatment has to be carried out. The opening up of the root is carried out with the crown of the tooth still standing until root treatment has been completed. This is to allow the gum still to stand free of the edge of the root, otherwise if the crown were cut off first, the gum would creep over and trespass on the face of the root, also the patient would prefer to have the crown standing rather than have a big space for some time. The crown, then, has to be cut off; some operators do this by cutting a nick on each side of it and using excising forceps to cut it off. This is a clumsy method, because it jars the periosteum to start with, and the face of the root is splintered, making a rough-edged joint. The better way is to take a small sized cross-cut fissure bur well dipped into bur lubricant and apply it to one side of the crown just under the enamel if possible, then with a sawing motion we cut it through like a lumberman cuts down a tree-trunk. The bur may have to be lubricated two or three times, as it gets clogged up and cannot cut, but engenders heat, which may break the shank of the bur. There are extra long fissure burs on the market which are suitable for this purpose, but they must be thin ones or the cutting will be a clumsy process for the patient. The extra length is useful as it allows of accommodation for a thick crown. We must be careful to hold the crown with the fingers when it is about ready to fall off. The face of the cut root must now be ground down so that its labial and lingual edges are just under the free edges of the gum, and yet these edges must not be merely bevelled, as it is impracticable to fit the porcelain crown down over them. The surface must be perfectly flat from one edge to the other. A square edged carborundum wheel is most suitable for this purpose, about $\frac{1}{8}$ inch thick and $\frac{3}{4}$ inch in diameter. A very small wheel of the same thickness may be used for finishing down the edges near the gum. If a dressing is put into the root to be left for a day or two a cap which is shaped like a small drawingpin may be warmed, with gutta-percha on to seal it in and to keep the edges of the gum away. If persistent weeping of blood occurs after cutting the crown off it may be stopped by applying a weak solution of trichloracetic acid, or, better still, by applying the electric cautery. The next stage is the enlargement of the root canal to accommodate the metal post which carries the crown. Porcelain crowns with the posts baked in 
are very convenient in some cases; but in many others it is better to fit the post first and try the crown on to see in which direction it should accommodate the bite. We take a flameshaped bur to open the orifice of the canal, but not to bevel it. Then follow on with a Gates-Gliddon drill, as shown in the chapter on Root Treatment. A root reamer is then used according to the size of the post which we decide to employ: The post should fit snugly but not accurately, so as to allow for a film of cement to hold it. The posts supplied by the manufacturers are made either of German silver, or nickel alloy, and gold. We prefer to make the post of I 6 carat gold to the size required for each case. We have found that a nickel alloy post often breaks just below the surface of the root, which causes a lot of time to be consumed in order to remove it and fit a new one. Since we have employed gold posts we have not had any trouble Before reaming out the canal it is advisable to test the length of it. This may be done by placing a small piece of rubber dam on to the upper part of a broach before passing it up the canal and then closing the rubber down to the face of the root and drawing the broach away carefully, which then gives us the length. Lay this down on the bracket table for reference. We take a piece of thick gold oval wire and taper it down to the required size and adjust the canal to it according to the general size and build of the tooth. The post should be longer inside the canal than the length of the crown, and the part that approximates to the face of the root should be thick, as that is the weak spot and where the strain comes. The post may be used as it is, or, better still, the orifice of the canal may be enlarged with a very large round bur kept for the purpose so as to accommodate a ring of size A gold round wire being soldered to the post. This ensures great strength where it is needed, and makes a good hold for the cement. The coronal end of the post is then carefully filed if it is too thick in order to fit into the hole of the porcelain crown; this must be quite parallel, otherwise the crown will work loose if it is at all tapered. We then take a pair of cutting pliers in one hand and the post, held in a pair of pliers, with the other and cut threads round it for the purpose of making a hold for the cement. The crown is carefully fitted to the root in the mouth or, if more convenient, an impression with plaster and a wax bite may be taken and the crown fitted in the laboratory. We prefer to fit it in the mouth. Now, if 
the edges of the crown overhang the edges of the root it must be definitely cut to the alignment of the angle of the surface, and not merely bevelled. If necessary, a large portion of the porcelain must be cut to secure a natural and artistic appearance (see Fig. 96). If Ash's or the Dental Manufacturing Company's Robbin's crowns are employed they may be cut to any extent to match the configuration of the neighbouring teeth. They can then be disked up with medium carborundum discs and finished with very fine cuttlefish or sandpaper discs. If a high polish is desired they may be finished on the lathe with a buff, using pumice and dry whiting. A cement of good quality must be used to fix it into the canal. During the whole operation the mouth must be kept

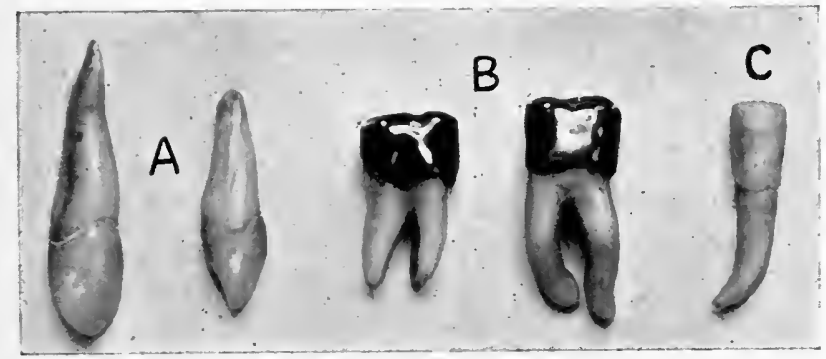

Fig. 96.-Specimens of Crown Work.

A, Porcelain crowns with surfaces ground and polished to make them flush with the contour of the roots; $\mathrm{B}$, gold shell crowns, showing shaped cervical edge; $\mathrm{C}$, lower bicuspid coned and cusps ground before making a crown.

quite dry with napkins, wool rolls, and the saliva ejector. The napkin will have to be swung out of the way occasionally to test the position of the crown and bite. When the post and crown are ready the canal is dried out with absolute alcohol and hot air. The periphery of the face of the root is cleansed with peroxide of hydrogen, then with alcohol, and well dried. Crown and bridge cement is then carefully mixed to the proper consistency, and with a fine plugger point we work a little into the hole of the porcelain crown, which also has been carefully cleansed and dried; smear some around the coronal end of the post and plunge it into the crown; then build more cement around the whole of the post and lay it down on the slab while we pass a little cement right up to the apex of the canal. This process is important, 
as we wish to medicate the end of the canal. We take a very fine plugger, catch up a little cement on to it, and dab it on to a small piece of thymol crystal which has been crushed before we mixed the cement. We only require a trace of this drug. We then pass this right up to the end and twist it round. The remainder of the cement is then placed into the root canal so as to avoid air getting locked in. Then take the tooth and post and plunge it without any force into the canal and pause about halfway to release the compression, and then push it home and hold it for a minute. When the cement is quite set the excess is trimmed away and the patient is directed to hold a little warm water in the mouth to complete the setting of the cement. The cementing of the post into the crown must be done first, because if the canal is filled first we shall find that the warmth of the mouth will cause it to set and thus spoil the whole thing. The apex of the canal may be sealed first if desired before the post is fitted, or the post may be cemented to place first, and then the crown fitted afterwards. We prefer this method because there is a certain amount of undesirable play when carrying post and crown into the canal together. The writer has fitted hundreds of porcelain and pivot crowns, and regards the fixing of them with a gold post of suitable size as an cntirely satisfactory operation, and one which may be carried out with every confidence of success as any other operation in dentistry. Years of experience with this method have shown that it contains the solution of the permanence of root filling which appears to cause so much controversy. The apex of the root canal and its foramina being the vital spot, slight medication, in proportion to the smallness of the risk of sepsis with solidity of the filling by condensation, secures its permanence. By this we mean that it is not necessary to hare a mass of medicament to take care of a tiny crevice which might contain the origin of sepsis. We can overwhelm the risk and make a much greater one because the porous nature of some filling materials allows the serum from outside the apex to permeate inside the canal. In this way accommodation is ultimately afforded for the harbouring of micro-organisms which infect the outside apical tissues, giving rise to granulomatous areas which can be detected in radiographs taken in suspicious cases. The root filling must be solid or impermeable in substance, there must not be any space or any tenuity as opposed to condensation. 
This condition is secured by the method which we have described. Upper and lower bicuspids are suitable for post crowns. Upper and lower molars are sometimes suitable for porcelain crowns by making a Richmond band or collar to the root with $\perp$-shaped pieces underneath in place of a post, and a post soldered to the crown side to take a tube tooth or a porcelain crown. In this case the gold cap is made to the root in the mouth, and a composition impression and bite is taken so that the post and crown may be finished in the laboratory.

For crowning molars and also bicuspids, where they are being utilized for bridgework as abutments, shell crowns are suitable. If there is a cavity in a tooth that is to be crowned in this way, the practice of relying upon cement to fill it when cementing the crown on is bad. The cavity should be faithfully filled with amalgam as if there were no crown to be fitted. This is one of the causes of leaking which finally leads to septic trouble under a crown or a bridge. Oxyphosphate of zinc is a necessary dental cement, and is much abused because too much is expected of it. The acids of the mouth will sometimes dissolve the cement, which, as it becomes removed, provides space for them to concentrate and remove the remainder more quickly. When the cavity is reached the process goes on which removes the cement filling until the whole thing becomes septic and hopeless bccause the bridge has to come off and the septic tooth extracted. If the cavity edges had been securely filled with amalgam the bridge could be cemented on again.

The next procedure after filling the cavity is to cone the tooth, which means that we must at least make the sides parallel so that the cervical edge of the gold collar or band fits snugly round, and yet will pass on and off while making the gold crown (see Fig. 96). This is carried out with a square carborundum wheel $\frac{1}{4}$ inch thick and $\frac{3}{4}$ inch in diameter; the smaller parts and bulbous surfaces are dressed down with smaller stones, or cup-shaped ones. If the tooth has one or two tecth immcdiately touching it we must make a space by trimming its approximal sides. This process involves great care in crder to avoid lacerating the gum, cheek, or the tonguc. There are many kinds of abrasive discs for this purpose, which meet the difficultics involved. In some cases the enamel is adamant, which, ccmbined with the tightness of the approximating teeth, makes it a difficult process. The disc will sometimes get suddenly wedged 
and then jump off suddenly, or it will break, leaving a jagged edge to run amok. The cutting of the soft tissues must be avoided, yet the tooth must be cut through. The quicker it is done the better the cutting will be. A firm, steady wrist is essential in order to control the engine handpiece, and practice brings the necessary skill. Students, when first using a bur, find that it will run about everywhere but where they want it; so with a cutting disc, if it is not steadily held it is out of control and does not cut as definitely as it otherwise would. The safest procedure is first to make a division with a fine steel ribbonsaw strip held with the fingers and followed if possible with a separating file. Knife-edged cutting discs and saws must be used with great caution. Vul-carbo discs and copper discs used with carborundum powder are as safe and efficient as anything can be for this purpose. However, with practice out of the mouth the nature of the process can be investigated. An experienced operator will do it in the mouth with speed, confidence, and skill. An assistant holding a water syringe and slowly dropping a little cold water on the disc will help the cutting. Having coned the tooth we proceed to grind down the cusps of the natural crown in order to make room for the top or biting portion of the gold crown. In doing this operation we find in live teeth with deeply defined cusps, that when we grind on the centre of them there is a decidedly sensitive spot. If we take a new, coarse carbo-stone and keep it well moistened with cold water we can cut them quickly and with a minimum of pain. A skilful manipulation of the carbo-stone is necessary to secure this dual purpose, and can be acquired by practice. The nearest guide to its acquisition is that the wheel should be applied with a side pulling action and not with a direct vertical pressure. Considerable power is developed by the sweep of the rapidly revolving stone with this slide slip movement.

The fitting of the collar is the next stage, which is very important. It cannot be done accurately enough on a plaster model, because the gum margin cannot be correctly shaped. The thickness of 22 carat gold should be No. 4 or 5 -No. 4 for single crowns and No. 5 for abutments on a bridge. The measurement of the tooth is taken with a dentimeter, which consists of a loop of binding wire held by a clamp. The loop is placed around the tooth, and the ends twisted up closely but not too tightly. It is removed and one side is cut through, 
closely against the twisted part, and straightened out, which gives us the length which is marked on the gold, allowing for a slight lap. The wire is then roughly cut and bent at a right angle to find the depth of the collar with relation of the lowest depth level of the gum to the morsal edge. This is then marked on the gold for its width. Bend the piece of gold, which has been cut to the ascertained measurements, so that the join comes in this case in the centre of the lingual surface, but for a bridge it is better to make it on the inner side so as to incorporate it in the solid part which forms the dummy crowns. Be careful to see that we form a parallel cylinder and make sure that the cervical edge fits closely. This is done by holding the band together with one hand while marking the joint lap with an instrument in the other. When the collar has been removed we can then tell where the joint has to be soldered. To remove the spring in the gold before boraxing it we grasp it with tweezers and hold it in a Bunsen flame first, to anneal it. Solder it with 22-carat gold solder or a suitable high carat solder and hold it in the Bunsen flame until it flows well. The band is then replaced on to the tooth and tapped with a crown punch and mouth mallet, but not heavily, and where the edge of the band is short it is marked, removed, and in this way it is finally trimmed, filed, or disked, until the edge is accurately fitted to the gum. The patient must not be hurt, as this indicates that we are injuring the periodontal membrane. If a band is forced at any point on to the pericementum it will give great pain and cause periostitis and lead to its absorption, which thus causes leakage at the cervical edge. It will now be seen that a crown band cannot be accurately fitted to a plaster model however accurate it may appear to be. The surfaces, particularly the buccal side, can be contoured with suitable contouring pliers. Nothing looks worse than a straight-surfaced crown, which looks more like an umbrella ferrule than a tooth. On the other hand, there are many crowns which look well shaped and sleek at the expense of the amount of gold that should be in them. Thin, paper-like crowns are not rigid enough to withstand the stress of mastication, and give way by buckling and becoming loose and perforated.

Having made the collar we now have to construct the top, which consists of making a joint with the collar and a biting surface to articulate with the antagonizing teeth and sufficiently 
strong and thick to do its work. There are several methods of making a top to a crown:

I. A quick method.

2. Striking up a top.

3. Casting a top.

4. The diaphragm method.

I. A suitable shaped top is selected on the crown die-plate, and a piece of No. I or No. 2 thickness of fine gold is struck into it with a large lead shot or a small piece of lead. It is then adjusted, if necessary, so that the contour of the top corresponds to that of the band. A piece of high carat solder is just tacked on one side and then on the other, but it must be a small piece. The crown is then put on the tooth and the patient bites well on to it. It is then taken off and the edges are soldered with a lower carat solder than was previously used, which prevents the other joints on the crown shifting in any way. Skilful work can be done by using various carat solders in crown work. The cusps of the crown are filled in with solder so that the crown neither becomes perforated through biting stress or buckled up. This method is suitable where there is plenty of room for the bite.

2. In some cases there is very little room available for the top owing to the extreme sensitiveness of the tooth surface. We must secure room at every part to take a No. 7 thickness of gold. We put the collar-band on to the tooth and place a small piece of soft composition on top and let the patient bite, then hold the compo up to the light, and if there is a hole, a little more tooth surface must be ground away. A piece more compo is then placed on to the tooth, and the patient bites again on to it. It is cooled with a jet of water and removed, also the band. The band should be marked so as to replace it in the compo correctly, and a model and bite made in the laboratory. A No. 7 thickness of 22-carat gold top is then struck with a zinc die into a block of lead mixed with a little tin, then soldered to the band and finished. Before casting a model for a crown, care must be taken to run a little marble dust and plaster (3 parts to 4) into the collar, because when the high heat is developed for soldering it, plaster alone will contract very much, which allows the solder to run in and encroach, thus causing the crown to be raised when fitted on the tooth. A crown that 
does not go home properly will cause undue pressure on the periodontal membrane, making it sore, and inflammatory trouble commences straight away.

3. A top may be carved suitably in inlay wax and cast, then soldered and finished. A crown may be nicely contoured by this method.

4. The strongest form of top which is suitable to stand the strain of a bridge is made by first securing a fair amount of room between the bite and the tooth surface. A diaphragm of fine gold is soldered to the top of the collar and placed on the tooth and tapped with the crown mallet into its place. In doing this an impression will sometimes appear of the surface underneath on the gold diaphragm. The crown top has now to be completed. We place a piece of compo and take an impression in the same way as for No. 2. A suitable top is selected from the die-plate and placed on the crown, and the bite adjusted. The top is marked with the collar so that we can tell where it goes when removed. The cusps are filled with high carat solder first and then placed on to the crown and held in position with a crown soldering clamp. It is now soldered solidly with a lower carat solder, which is drawn right through. The object of filling the cusps with a high carat solder first is to prevent their becoming hollow when the final filling in is executed. The crown is then trimmed up and completed.

We have indicated the main features of crown work but, as we have already stated, it is capable of endless variety and combination of details. It provides a wide field for the ingenuity of a good mechanic and the subtle finish of an artist. The utilization of two or more crowns may form the piers or abutments of a bridge. When these have been properly made and fitted into position, but not cemented, we proceed to take a bite with wax. An impression is then taken with plaster of Paris to include the field of the bridge. This may be a partial impression or a complete one of the jaw. The plaster may have to be fractured in order to remove it, and the crowns and broken parts placed very carefully together in the tray and waxed into position. A model and bite is then made in the laboratory, and the spaces between the crowns are filled in with platinum pin teeth or interchangeable teeth facings, and finally soldered into one piece with strengthening bars adjusted previously to prevent contraction taking place from the solder. 
This part of crown work comes into the domain of mechanical dentistry and is an extensive subject. Removable bridgework provides a still greater field for variety, ingenuity, and really accurate work. It has the advantage of providing the patient with a comfortable denture which is secure in its place with a minimum of size so as to allow plenty of room for the tongue. It also allows of proper cleansing.

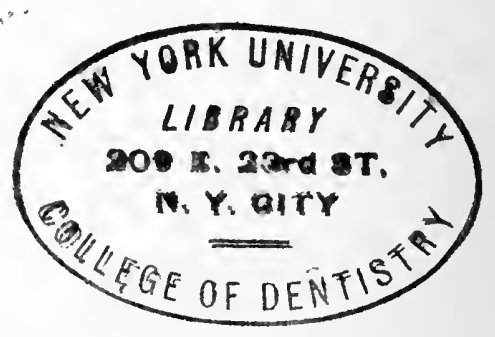




\section{INDEX}

Abscess, 147

dental, I 59

Aconite tincture of, 184

Adrenalin, I 75

Alcohol, I 75

Alum, 182, 233

Alveolar process, 60

Alveoli, formation of, 34

Amalgam, condensation of, 289 fillings, 283,285 formulæ, 284

Ameloblasts, 22

Ammonia bifluoride, I 85

Anæmia, 204

Anæsthesia, Dr. Gwathmey on, $30_{4}$ pressure, $3 \mathrm{O}_{4}$

Anastomoses, 81

Aneurism, 204

Anti-thrombin, 103

Antral suppuration, I 54

Antrum, description of, 60 empyema of, 60 importance of, 63

Apical foramina, 299

Arachnoid membrane, II 4

Aromatic sulphuric acid, I 86

Arsenic, I 81 action and use of, 303, 304, 306, 307

Arteries, anastomoses of, 74 description of, 74 distribution of, 74

Arterioles, 78 function of, 89

Arterio-sclerosis, 74, 204

Artificial respiration, 222

Asphyxia, 84 causes of, 93 stages of, 94

Bacteria. I 50

Bacteriology, I 49

Basement membrane 65

Bobœuf's Phénol Sodique, 20I, 232

Buckley's phenol comp, I8o

Bur lubricant, 266

Blood, absorption of gases, 84 arterial, 7 I

bactericidal power of, 99

description of, $\delta_{2}$
Blood, carbonic acid gas in, 84

coagulation, reason for, 97

coagulation of, IOO affected by certain conditions, IO2

composition of, IOI

corpuscles, description of, 83

formation of clot, IOI

function of, 68

immunity of, 97

mechanism of circulation, 88

oxygen in, 84

percentages of gases in, 9I

plasma, 83,86

platelets, 83

power of, in combating disease, 98

pressure, 88,95

process of coagulation of, $\mathrm{IO}_{2}$ proteins in, 86

resistance of, $\mathrm{I}_{47} 7, \mathrm{I}_{5} 8, \mathrm{I}_{70}$

stream, velocity of, 89

supply of teeth, 49 to teeth, 126

temperature of, 82

venous, 7 I

Bloodvessels of mouth, 66

Bone, chemical composition of, 50 qualities of, 49

structure and characterístics of, 5 I

Bones, cranial, 52

facial, 52

function of, $5^{\circ}$

of the skull, 52

Brain, connective tissues of, II 5

coverings of, $1 \mathrm{I}_{4}$

description of, 108

divisions of, I I I

functions of, Io8

neuroglia of, I I 5

ventricles of, II 2

Bright's disease, $2 \mathrm{O}_{4}$

Calcium salts, in coagulaiion of the blood, $\mathrm{IO}_{4}$

sulphide, I 79

Camphenol, I $84,201,232$

Capillaries, 78

Carbolic acid, I 79 
Carbolized resin, I 80

Caries, Dr. Register on, 264

Cartilages, capsular, I 36 external lateral, I.36 interarticular fibro-, I 36 internal lateral, ${ }_{3} 6$ stylo-mandibular, ${ }_{3} 6$

Cavernous sinus, I 59

Cavities, classification of, 263 defining limits of, 267

discovery of, 262

lining of, 273,285

plugging of, 273 preparation of, 264

Cavity dressings, $27 \mathrm{O}, 27 \mathrm{I}$ preparation, pain in, 279 methods of securing dryness, $280,28 \mathrm{I}$ vulnerable parts, 285

Cell, the, 7 I action of drugs on, 172 description of, I 5

Cells, origin and development of, 70

Cementoblasts, 48

Cementum, 28 description of, 45

Central nervous system, 106

Cerebellum, II 2

Cerebral cortex, I I 5

Cerebrum, II I

Children's teeth, fillings in, 239

Chinosol, I 83 regulation of, 249

Chloretone, I 78 , I 93

Chloride of calcium, I 79

Cinnamon oil, I 77 and tannin, 269

Circulation, portal, 80 pulmonary. $7 \mathrm{I}$ systemic, 80

Cocaine, I9I

poisoning, symptoms of, 200

Comparative anatomy, advantages of, 49

Copper sulphate, I 85

Corium, 65

Corpora quadrigemina, I I 2

Corpus callosum, I I

Cortex, convolutions of, I 6

Creosote, I 88

Cresylic acid, I $\delta_{4}$

Crown and bridge work, causes of failure, 323

plaster of Paris impressions, 333

Crown work, 323

cutting divisions between teeth, $33^{\circ}$

registering length of root canal, 326
Crowns, gold shell, 329

porcclain, 326 cementing of, 327

shell, fitting of collars, and soldering tops, $33 \mathrm{I}$

Crura cerebri, II 2 , I I 9

Deglutition, I 38

Dental abscess, drainage of, 3 I 8 treatment of, 300

arch, contraction of, $25^{\circ}$

caries, origin of, 264

follicle, 20

follicles, 30

periosteum, 28

pulp, 28

description of, 44 origin of, 27

Dentalone, I 78

Dentinal papillæ, 20

Dentine, calcification of, 22

carious, stages of, 269

chemical composition, 44

cutting of, 267

decalcified, tanning of, 269

description of, $4^{\circ}$

Dentition, 249

abnormal symptoms of, 37

normal symptoms of, 37

vagaries of, 38

Devclopment of size of jaws to accommodate the teeth, 248

Digitalis, 93

Dislocation of lower jaw, 56, I 37

treatment of, I $60,16 I$

Drugs, classification of, 173 used in dentistry, I 74

Dura mater, II 4

Effect of respiration on circulation, 89

Electric cautery, use of, 280

Eminentia articularis, 53

Empyema, I 47

Enamel, calcification of, 22, 27 chemical composition of, $4^{\circ}$ cutters, Dr. Black's, 265 cutting, 265 description of, 38 organs, 19,20 rods, 265,266

Endocarditis, 203

Enzymes, I 49 importance of, 103

Epiderm, 16

Epithelium, 65 band, i 8

Epithelial cells, I 7 cords, I9, 23 
Epithelial cords of deciduous teeth, separation of, 24

of successional teeth, scpatissue, 16 ration of, 25

Epulis, $16_{5}$

Erythrocytes, $83^{\circ}$

Erythrophleine sulphate, I 8I

Essential oils, 177

Etiology, r 44

Eugenol, 178

Exostosis of roots, 28, 166

Extraction of teeth, difficult cases, 228

movements for, 226

preparatory treatment, $23 \mathrm{I}$ principles of, 223

Fainting, 73

Fibrin, I0x, 104

Fibrinogen, 86, Io.

Fibroblasts, 48

Fillings, condensation of, $27+$

Fistula, I 59 irritation caused by, 273,278

Foramen magnum, I 9

ovale, 55

rotundum, 55

Foramina, Scarpa's, $6+$ Stensen's, 64

Forceps for extraction, 224

Formaldehyde, I 82

Frontal process, 60

Gasserian ganglion. 55, 120

Gingivitis, 164

Glenoid cavity, 53

Glycerine, I 82

Goitre, $2 \mathrm{O}_{4}$

Gold, cohesive, 293

crystal, 294

filling, 292

causes of failure, 295

non-leaking margins, 294 , 295

forms of, for filling, 292

non-cohesive, 293

pluggers, 296

Gum, hypertrophied, 280

Gums, description of, 64 . hypertrophy of, 164

Hæmatoma after extraction of teeth, 23I

Hamoglobin, 84, 91

Hæmorrhage, treatment of, 232, 233

Hæmorrhagic diathesis, roo

Heart, function of, 68 description of, 68,72

hypertrophy of, 75

nervous control of, 72
Heart sounds, cause of, 72 valves, 72

Hewitt, Sir Frederick, on nitrous oxide and oxygen gases, 2 ro

Hyperæmia, 145

Hypertrophy of tissue, I 48

Hypochlorites of calcium and soda, I 86

Hypoderm, 16

Hypodermic armamentarium, I 94 injections, principles of, I 97 syringes, 195

Induration, $\mathrm{I}_{4} 8$

Infection, $\mathrm{I}_{4} 6$

Inferior dental canal, 59 maxilla, description of, 55

In flammation, I 45 chronic, 146 resolution of, 146

Interchange of gases, 90

Invagination of dental follicle, 29

Iodine, 183

[rritation, I 45

Isotonic solutions, 194

Jaws, 30

development of, $2 S, 29$

fracture of, I 60

origin of, 18

Larynx accidents to, I 42

Leptothrix buccalis, I 57

Leucocytes, 85 , I 59

function of, 86

Lymph, description of, 87

Lymphatic system, 67 vessels, I 54

Malar bone, description of, 52

Malpighian layer, i 8, 25 lining, 65

Mastoid process, 53

Matrices, fitting of, 287

and matrix clamps, author's system of, 289 importance of, 277,287

Maxillary sinus, 60

Meckel's cartilage, I 8, 30

Medulla oblongata, description of, I I $2,113,119$

decussation of pyramids, I I 9

Mental foramen, 59

Menthol, $x 87$

Mesoderm, I 6

Metabolism, 69

Molars, origin of, 25, 26

Morbid histology, 144

Moylan-Jones gas and oxygen apparatus for nasal anæsthesia, 212 
M'גวว

disenses of, 163

Mummifying paste, 308

Muscles attached to mandible, 59

b:accinator, I 33

contrastion of, 162

depres3or anguli oris, I 3 I labii in ferioris, I $3 \mathbf{I}$

hyo-glossus, I 39

importance of non-interfarence with, 228

levator anguli oris, $x_{30}$

labii supzrioris propitus, I 30 menti, I 3 I

misseter, I 33

mylo-hyoid, I 39

orbicularis oris, 130

pterygoid, external, 55. I 35 internal, 55, I 35

risorius, I $3 \mathbf{I}$

stylo-glossus, I 39

temporal, I 35 origin of, 53

zygomaticus major, 130 minor, I3I

Necrosis, 158

Nerve fibres, 107

force, II 7

Nerves, afferent or centripətal, iro cerebro-spinal, ro6

depressor, I 26

efferent or centrifugal, rog

filth puir, r 20

function of, ro8

inferior maxillary, 124

intercentral, I Io

Meckel's ganglion, $\mathrm{I} 2 \mathrm{O}$

motor, 109

naso-palatine, I2I

pterygo-palatine, I 2 I

sensory, I Io

seventh pair, 1.56

spheno-palatine, I 20

superior maxillary, I 20

sympathetic, 106, 125

vagus or tenth pair, 125

vasomotor, 12.5

Nervous system, mechanism of, ro-

Neuralgia, 156 peripheral, 106

Neuroglia, Io7

Nitrate of silver, I 88

Nitrous oxide gas anasthesia:

apparatus and instruments, 204

combination witl oxygen, 208 nasal administration, 216,219 prevention and treatment of accidents, 208, 222
Nitrous oxide g 13 an e; th ssia: signs of anæithesia 207 stages of anxithosia 236,23 Normal saline solution, I9t

Olontoblasts 22,38

Otontoma $\mathrm{I}_{3}$

Oil of cassia. 178 of wintergreen, I 80,233

Opən bite, 249

Osmosis, 93. 91, I94

Osteo-arthritis 138

Osteoblasts, 28, $4^{8}$

Osteoclasts 48

Oval burs. importance of, 253

Oxygen, 235

Palatal bones dezcription of, 6 , process, 6)

Palatine canals, 66 anterior, 63, I 21 posterior, 63

Paramonochlorphenol, I 8I

Parotid gland, I 40

Parotitis, I 57

Perchloride of iron, 175

Pericementum, 47

Peridental membrane 47

Periodontal mombranz, $2 \mathrm{~S}$

PerioJontitis, I65

Periosteum 47

function of, $5 \mathrm{I}$

Peripheral resistance, 89, 93

Peroxide of hydrogen, I74

Petrous portion of temporal bone, $\mathbf{5 j}$

Phagocytes, 98

Phagocy tosis, 86, 99

Phenate of soda, 18 J

Phenol. I 79 sulphonic acid, I 86

Physiology of respiration, Dr. Halliburton on, 205

Pia mater, II 4

Polypus, $16_{5}$

Pons Varolii, I I 2, I I 9. I20

Proliferation of cells, I 45

Protoplasm, I5, 69, 85, I I 7

Protrusion, 249

Pterygoid plates, 55

Pterygo-palatine canal, 12 I

Ptomaines, I 49, I 50

Ptyalin, I4I

Pulp, anatomy of, 299 calcareous deposits of, 313 devitalization of, 298

Pulse, description of, 76 in disease, 76 effect of tobacco on, 77 intermittence of 77 irregularity of, 76 practical use of, 76 
Pulse, table of, 78

Pus, drainage of, I 59 how produced, 147

Pyæmia, I 48

Pyorrhœa, seriousness of, I 68 signs of, 166

Ranula, I 57

Reflex action, I I 5, I I 7, I I 8

Regulation of teeth, principles of, $25^{\circ}$

Repairing of sockets after cxtraction, 52

Respiration, function of, 90 table of, 78

Responsibility of operator, 46

Restoratives, 200

Ringer's solution, 194

Root canals, opening up of, 3 Or, 3 I I use of paper points in, 302

filling, medication of apex, $32 \mathrm{~S}$

fillings, 314,322 author's formula, 3 I 5

treatment, 298 application of dressings, 321

dead and septic teeth, 316

Roots, calcification of, 30 principles of, 316,317

development of. $3^{\circ}$

opening up of, for crown work, 325

Rubber dam. 295 application of, 282,283

Saliva, chemical composition of, $1+1$ Salivary glands, $\mathrm{r}_{4} \mathrm{O}$

Scaling teeth, 262

Secondary infections, 147,160

Septicæmia, $\mathrm{I}_{4} 8$

Septic thrombosis, I 59

Serum, IoI

Silicate cements, 274,276

Sphenoid bone, description of, 55

Sphenoidal fissure; 55

Spheno-maxillary fissure, 64 fossa, I 2 I

Spinal cord, functions of, 108 ganglia, ro7

Spirochxta pallida, I 56

Squamous portion of temporal bone, 5.3

S. S. White's gas and oxygen apparatus for nasal anæsthesia, 220

Staphylococcus, I $\$ 7$

Stasis, I 47

Stensen's duct, I $4^{\circ}$

Stomatitis, I 67

Streptococcus, $\mathrm{r}_{47}$

Strophanthus, 93

Styloid process, 54
Stylo-mandibular ligament, $5 \cdot t$

Sublingual gland, $14^{\circ}$

Submaxillary gland, $44^{\circ}$

Submucous tissue, 65

Successional teeth, origin of, 23

Sulphuric acid, r 85

Superior maxilla, description of, 59

Suppuration, I $_{4} 6$

Surfaces of teeth, names of, 263

Sylvius, aqueduct of, I 12

Symphysis of lower jaw, 29

Symptomatology, I 44

Syncope, 73

Synovial membranes, 136

Syphilis, signs of, I 55

Systems of the body, 69

Tannin, 176

Teeth, deciduous, characteristics of, 30

eruption of, 3 I

periods of eruption, 35

successional, 28

superadded, 28

table of eruption, 36

Temperature of body, 78

in relation to pulse, 79

Temporal bone, description of, 53

Temporo-mandibular articulation, I 36

Teter, Dr., on the nasal administra. tion of gas and oxygen, 209

Therapeutics, dental, I 7 I factors in, 172

Thoracic pump, 90

Thrombin, ro 3,104

Thrombogen, $\mathrm{IO}_{4}$

Thrombokinase, $10_{4}$

Thymol, I 82, I94

Tissues, nature of, $7^{\circ}$

Tongue, importance of the, I $\mathbf{5 5}$

Tonsilitis, I 55

Tooth crypts, 30

Topical anodyne, application for extraction of septic teeth, 201

Toxins, danger of, $x_{4} 8$

Trichloracetic acid, 186

Tumefaction, $I_{4} 8$

Tumours, I63, I65

Ulceration, $\mathrm{I}_{4} 8$

Ulcers, $\mathbf{I}_{4} 8$ of the tonguc, I 55

Vascular system, 67

Vaso-constrictor nerves, 92

Vaso-dilator nerves, 92

Vaso-motor centre, 93

system, 89, 92, 93, II 9 
Veins, 78

description of, 80 pulmonary, $8 \mathrm{r}$

valves of, $8 \mathrm{I}, 82$

Wharton's duct, I 40

Wool roll clamps, 282
Zinc chloride, 185

oxide, $\mathrm{I}_{3}$

oxyphosphate of, cement, $27 \%$. 275

sulpho-carbolate, I 80

Zygomatic fossa, 53

process, 53,60

Zymogen, importance, of, 104 

UNIVERSITY OF CALIFORNIA LIBRARY

Los Angeles

This book is DUE on the last date stamped below.

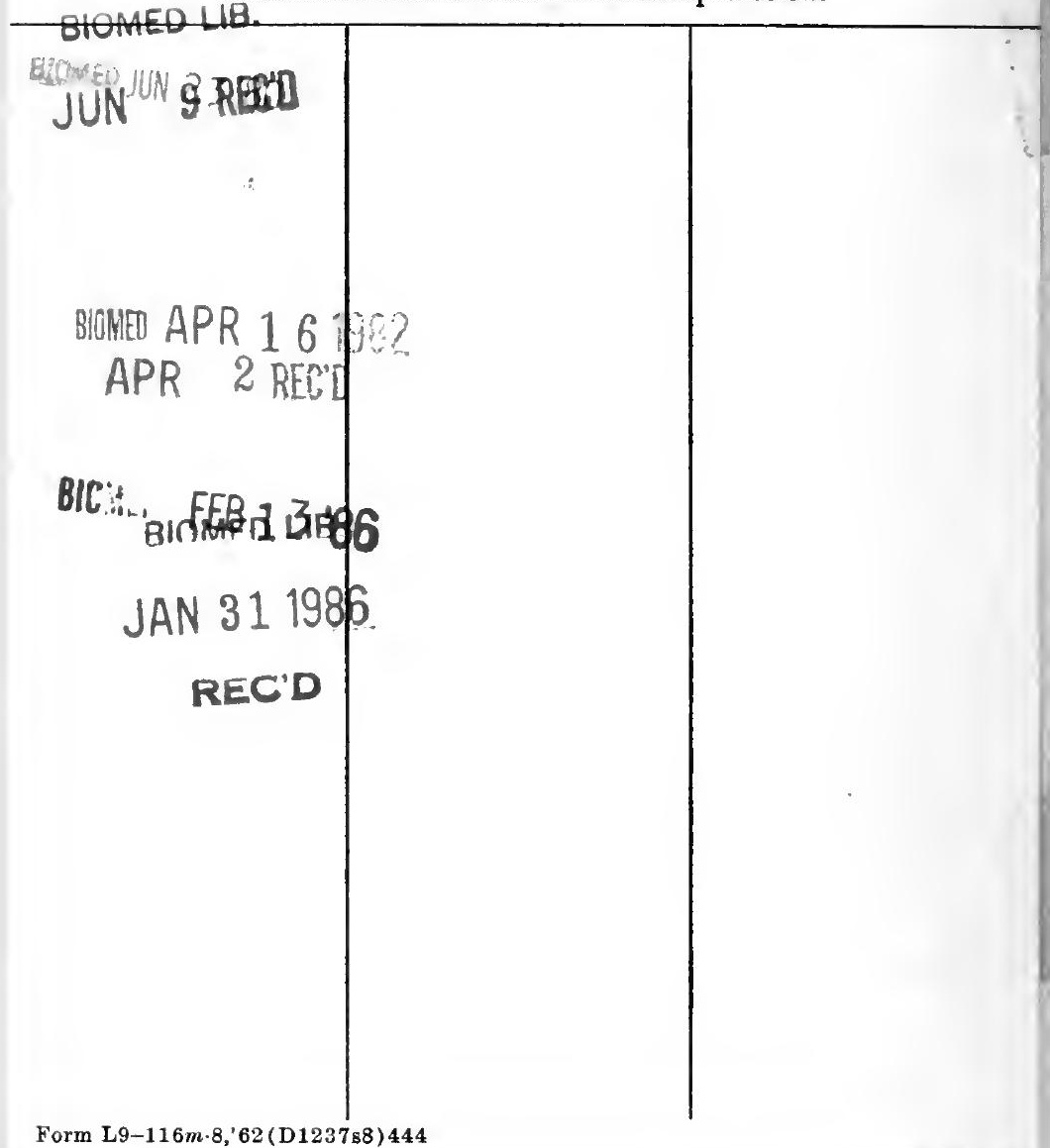

Form L9-116m.8,'62(D1237s8) 444 


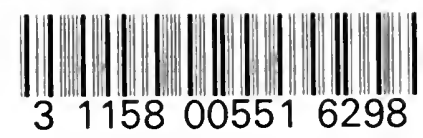

Goochuch

Operative Dental Surgery
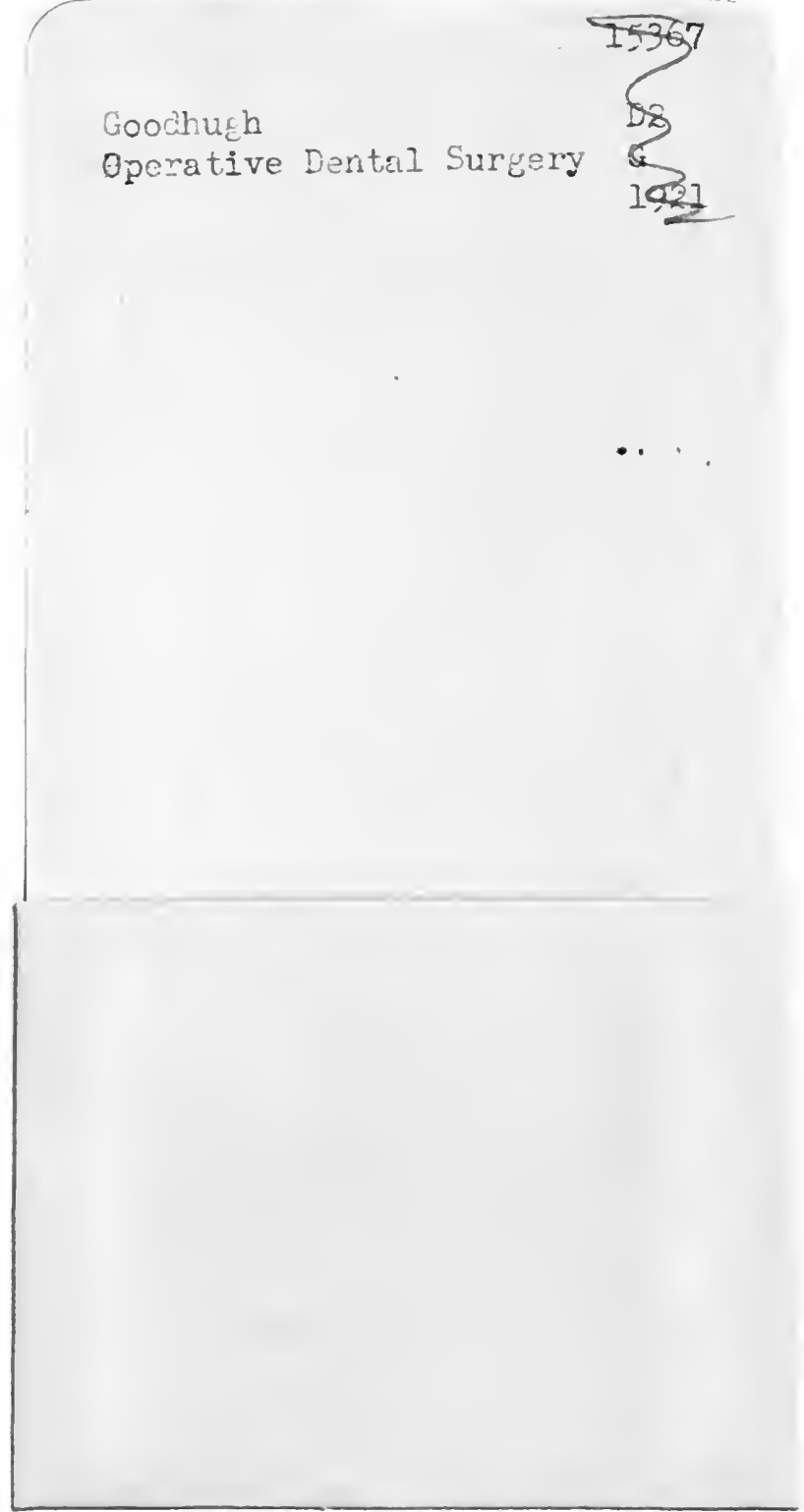
